\title{
Circulating Matrix Gla-protein
}

Citation for published version (APA):

Cranenburg, E. C. M. (2011). Circulating Matrix Gla-protein: a biomarker for vascular disease. [Doctoral Thesis, Maastricht University]. Maastricht University. https://doi.org/10.26481/dis.20110317ec

Document status and date:

Published: 17/03/2011

DOI:

10.26481/dis.20110317ec

Document Version:

Publisher's PDF, also known as Version of record

\section{Please check the document version of this publication:}

- A submitted manuscript is the version of the article upon submission and before peer-review. There can be important differences between the submitted version and the official published version of record.

People interested in the research are advised to contact the author for the final version of the publication, or visit the DOI to the publisher's website.

- The final author version and the galley proof are versions of the publication after peer review.

- The final published version features the final layout of the paper including the volume, issue and page numbers.

Link to publication

\footnotetext{
General rights rights.

- You may freely distribute the URL identifying the publication in the public portal. please follow below link for the End User Agreement:

www.umlib.nl/taverne-license

Take down policy

If you believe that this document breaches copyright please contact us at:

repository@maastrichtuniversity.nl

providing details and we will investigate your claim.
}

Copyright and moral rights for the publications made accessible in the public portal are retained by the authors and/or other copyright owners and it is a condition of accessing publications that users recognise and abide by the legal requirements associated with these

- Users may download and print one copy of any publication from the public portal for the purpose of private study or research.

- You may not further distribute the material or use it for any profit-making activity or commercial gain

If the publication is distributed under the terms of Article $25 \mathrm{fa}$ of the Dutch Copyright Act, indicated by the "Taverne" license above, 
Circulating Matrix Gla-protein:

a biomarker for vascular disease

Ellen C.M. Cranenburg 
Cover and lay-out: Jveproducties

Printed by: Datawyse BV / Universitaire Pers Maastricht

(C) Copyright E. Cranenburg, Maastricht 2011

ISBN 9789461590084 


\title{
Circulating Matrix Gla-protein: a biomarker for vascular disease
}

\author{
PROEFSCHRIFT \\ ter verkrijging van de graad van doctor \\ aan de Universiteit Maastricht, \\ op gezag van de Rector Magnificus, \\ Prof mr. G.P.M.F. Mols, \\ volgens het besluit van het College van Decanen, \\ in het openbaar te verdedigen \\ op donderdag 17 maart 2011 om 14.00 uur \\ door \\ Ellen Cornelia Maria Cranenburg
}

geboren te Boxmeer op 22 januari 1982

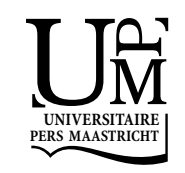




\section{Promotor:}

Prof. dr. J. Rosing

\section{Copromotor:}

Dr. C. Vermeer

Dr. L.J. Schurgers

\section{Beoordelingscommissie:}

Prof. dr. H. ten Cate (voorzitter)

Prof. dr. A. Bast

Prof. dr. J. Flöge (Rheinisch-Westfälische Technische Hochschule Aachen)

Prof. dr. J.M.J.P. van der Linden

Prof. dr. Y.T. van der Schouw (Universitair Medisch Centrum Utrecht)

Financial support by VitaK BV and FrieslandCampina for the publication of this thesis is gratefully acknowledged. 
Shoot for the moon.

Even if you miss, you will land among the stars.

- Les Brown -

Aan mijn ouders

Voor Arthur 



\section{Contents}

Chapter 1 General Introduction 1

Chapter 2 The circulating inactive form of Matrix Gla-protein (ucMGP) 25 as a biomarker for cardiovascular calcification

Chapter 3 Total uncarboxylated Matrix Gla-protein (t-ucMGP) is associated with coronary artery calcification in hemodialysis patients

Chapter 4 Circulating total uncarboxylated Matrix Gla-protein (t-ucMGP) is not associated with mortality in dialysis patients

Chapter 5 Characterisation and potential diagnostic value of circulating matrix Gla-protein (MGP) species

Chapter 6 Rare mutations in the gene encoding matrix Gla-protein (MGP): a new case of Keutel syndrome serves as a model for exploring MGP functions in humans

Chapter 7 Vitamin K intake and (hepatic and extra-hepatic) vitamin K status in hemodialysis patients: a pilot study

Chapter 8 General Discussion

Samenvatting

Summary

Curriculum vitae

Publications

Dankwoord 



\section{CHAPTER 1}

\section{General Introduction}

Ellen C.M. Cranenburg, Leon J. Schurgers, and Cees Vermeer

VitaK and Cardiovascular Research Institute Maastricht (CARIM), Maastricht University, Maastricht, the Netherlands

Based on: Thromb Haemost 2007; 98(1): 120-5 and Thromb Haemost 2008; 100(4):

593-603 


\section{Matrix Gla-protein}

\subsection{Background}

Matrix $\gamma$-carboxyglutamate (Gla)-protein (MGP) is a small secretory protein that can undergo two post-translational modifications: $\gamma$-glutamate carboxylation and serine phosphorylation. The protein was first described by Price and co-workers, who purified it from the bovine bone matrix [1]. The authors concluded that this approximately $15 \mathrm{kD}$ protein contained five unusual amino acids, which were designated as $\mathrm{\gamma}$-carboxyglutamate(abbreviated as $\mathrm{Gla}$ ) residues. This matrixassociated, Gla-containing protein was named matrix Gla-protein (Figure 1). After its discovery in bone, MGP was found to be expressed in several other tissues, including cartilage, lung, heart, kidney, and in vascular smooth muscle cells (VSMC) of the arterial wall [1-3].

Figure 1: Matrix Gla-protein
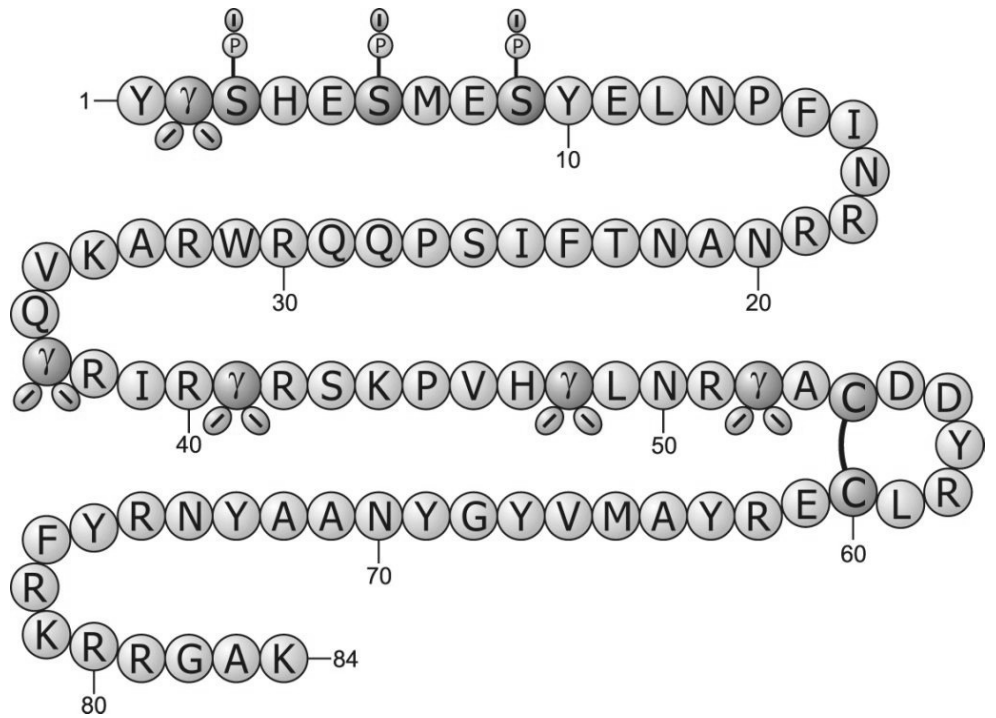

Matrix Gla-protein (MGP) is a small 84 amino-acids vitamin K-dependent protein. It can undergo two posttranslational modifications: at positions $2,37,41,48$, and 52 the glutamic acid residues (E) can be $\gamma$-carboxylated (indicated with $\gamma$ ) and at at position 3, 6, and 9 the serine residues (S) can be phosphorylated (indicated with $\mathrm{P}$ above). One disulfide bond is present, linking two cysteines (C).

Mature MGP consists of 84 amino-acids and has a theoretical isoelectric point of 9.7. The MGP gene is located on chromosome 12 (p13.1-p12.3), consists of four exons and three large introns and has a length of $3.9 \mathrm{kB}$. It presents putative binding sites for transcription 
factors AP-1 and AP-2, and for CAMP-dependent transcription factors. The fat soluble vitamins $A$ and $D$ can modulate MGP expression, their effect depending on the cell type.

\subsection{Post-translational modifications}

MGP can undergo two post-translational modifications:

1. $\mathrm{Y}$-Glutamate carboxylation

Of the nine glutamic acid (Glu) residues present in MGP, only five can be $\gamma$-carboxylated in a vitamin K-dependent reaction, resulting in the formation of Gla residues. These residues are located at position 2, and at positions 37, 41, 48, and 52 within the so-called Gladomain (amino-acid sequence 35-54).

\section{Serine phosphorylation}

MGP contains five serine (Ser) residues, of which three can be phosphorylated. This results in the formation of phosphoserine (Pser) residues, which are located within the amino-acid sequence 3-15 (positions 3, 6, and 9).

The best studied post-translational modification of MGP is $\gamma$-glutamate carboxylation, for which the energy is provided by vitamin K (Figure 2). The Gla residues formed are negatively charged and possess calcium-binding properties. They are required for protein activity of the vitamin K-dependent proteins (also called Gla-proteins) [4]. In all Gla-proteins the affinity for $y$-glutamyl carboxylase (GGCX), the enzyme necessary for carboxylation, is determined by a pro-sequence located immediately at the $\mathrm{N}$-terminal site of the protein. In most Gla-proteins, the pro-sequence is cleaved off during maturation. MGP is the exception in this respect, since the mature protein contains an internal pro-peptide. This may contribute to the unique properties of MGP.

Phosphorylation is thought to be carried out by the Golgi casein kinase [5, 6]. Price et al. showed that the motif in MGP recognized for serine phosphorylation is the tandemly repeated Ser-X-Glu sequence [5]. Wajih et al. showed that phosphorylated MGP exits VSMC via the secretory pathway, whereas non-phosphorylated MGP appears in the cytosol and is thus not secreted in the extracellular matrix [6]. Although the precise function of serine phosphorylation is not known, it may regulate MGP secretion into the extracellular environment. 
Figure 2: $\mathrm{\gamma}$-Glutamate carboxylation and vitamin $\mathrm{K}$ cycle<smiles>CNC(CC(=O)O)C(C)=O</smiles><smiles>CN[C@@H](CC(C(=O)O)C(=O)O)C(=O)O</smiles>

Glu

Gla

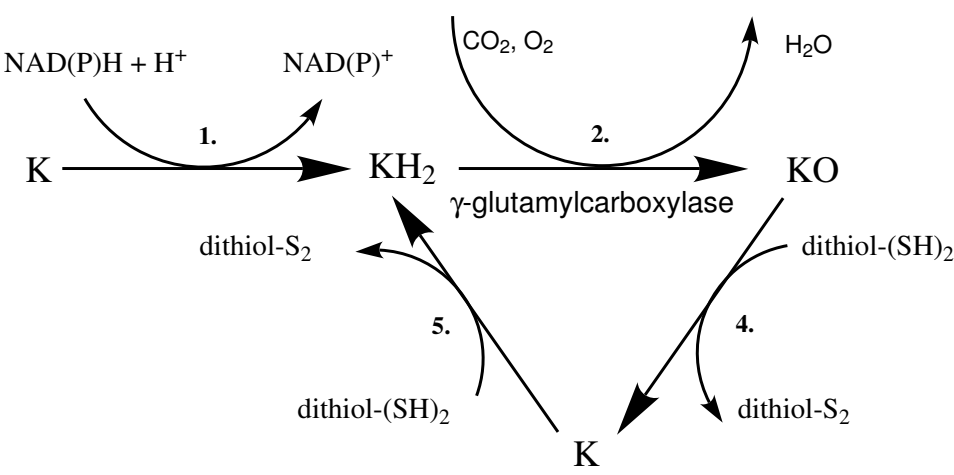

Vitamin $\mathrm{K}$ serves as a co-factor for the conversion of specific glutamic (Glu) acid residues into $\mathrm{Y}$-carobxyglutamic acid (Gla) residues. This reaction is linked to the enzymatic vitamin $\mathrm{K}$ cycle, in which vitamin $\mathrm{K}$ is salvaged for reuse in the carboxylation reaction. Vitamin $\mathrm{K}$-quinone $\left(\mathrm{K}\right.$, dietary form) is first reduced to vitamin $\mathrm{K}$-hydroquinone $\left(\mathrm{KH}_{2}\right)$. The active form $\mathrm{KH}_{2}$ is needed in the oxidation step, in which Glu residues are reduced to Gla residues. As a consequence of this reaction, $\mathrm{KH}_{2}$ is oxidized to vitamin $\mathrm{K}$ epoxide (KO). $\mathrm{KO}$ can subsequently be reduced to $\mathrm{K}$, and eventually again reduced to $\mathrm{KH}_{2}$. Many studies favour the assumption that the latter two steps are carried out by the same enzyme, vitamin $\mathrm{K}$ epoxide reductase (VKOR). Coumarin derivatives inhibit the enzyme VKOR, thereby blocking the vitamin $\mathrm{K}$ cycle and leading to a build up of $K O$ in the cell. However, in the liver the NAD(P)H-dependent quinone reductase (known as DT-diaphorase) can serve as an alternative pathway. This pathway can convert only $\mathrm{K}_{\text {into }} \mathrm{KH}_{2}$, and not $\mathrm{KO}$ into $\mathrm{KH}_{2}$. 1=NAD (H)-dependent reductase, 2=p-glutamylcarboxylase enzyme (GGCX), 3=extra introduced carboxy-group, 4=dithiol-dependent KO-reductase (VKOR), 5=dithiol-dependent K-reductase (VKOR) 
MGP exists as various species according to their state of phosphorylation and/or carboxylation: phosphorylated (pMGP), non-phosphorylated (desphospho, dpMGP), carboxylated (cMGP), or uncarboxylated (ucMGP). Development of antibodies directed against specific MGP species (Table 1) and of MGP assays to measure circulating MGP species (Figure 3) has enabled the investigation of these species in tissue (paragraph 1.4) and in the circulation (paragraph 1.5).

Table 1: Species-specific MGP antibodies

\begin{tabular}{lllll}
\hline Antibody & Directed against & Amino-acid & Specificity & Used \\
mAb-ucMGP & uncarboxylated MGP & $35-49$ & IgG I a & W, I, S, E \\
mAb-cMGP & carboxylated MGP & $35-54$ & IgG I a & W, I, S, E \\
mAb-dpMGP & desphosphorylated MGP & $3-15$ & IgG I a & W, I, S, E \\
mAb-pMGP & phosphorylated MGP & $3-15$ & IgG I a & W, I, S, E \\
\hline
\end{tabular}

Abbreviations used:

mAb, monoclonal antibody; W, Western blot; I, Immunoprecipitation; S, Section staining; E, ELISA.

Figure 3: MGP ELISA

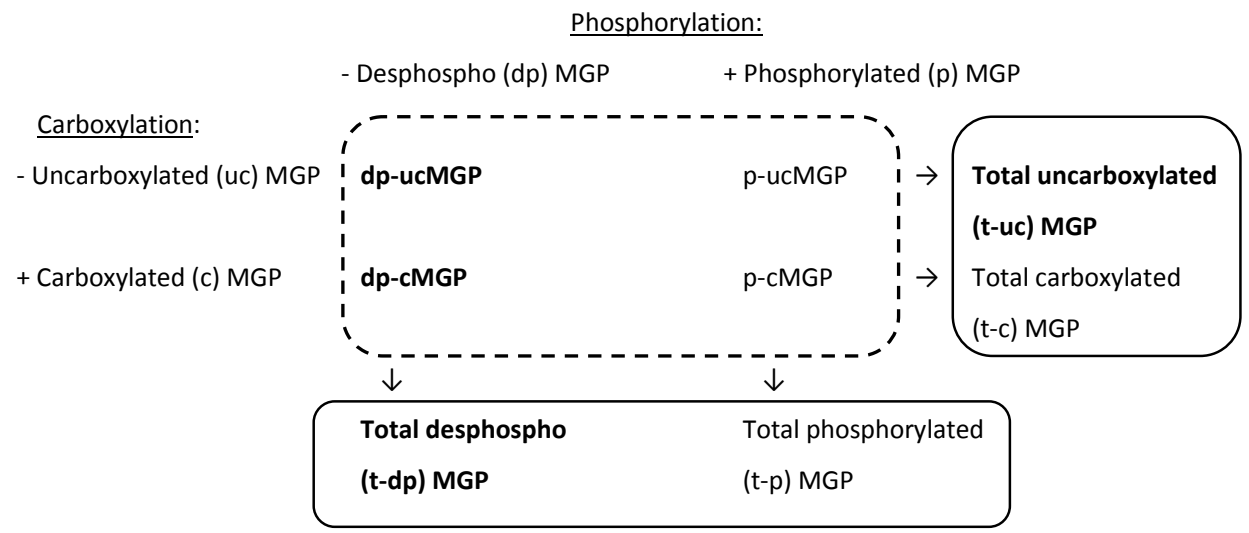

The following ELISA are depicted:

Single-antibody (competitive) ELISA (lined areas)

Four single antibody ELISAs could be developed, measuring ucMGP, cMGP, dpMGP, and pMGP. The first MGP ELISA described in the literature measures total desphosphorylated (t-dpMGP) and is commercially available (Biomedica, Vienna, Austria). The second MGP ELISA described measures total uncarboxylated (t-ucMGP) and its characterization and application in several patient populations is described in this thesis (Chapters 2-4).

Dual-antibody (sandwich) ELISAs (dotted area)

Combinations of the conformation-specific antibodies can be used to develop several MGP sandwichcombinations (in dotted area). Two novel dual-antibody ELISAs are described in this thesis (Chapter 5).

The MGP ELISA indicated in bold have been developed and used in the research described in this thesis. 


\subsection{Functions}

Although the precise molecular mechanisms of MGP function are not known, accumulating data demonstrate its major role in the inhibition of soft tissue calcification. The first indication that a Gla-protein was involved in the inhibition of tissue calcification came from rats treated with the vitamin K-antagonist warfarin [7]. These animals developed extensive cartilage calcification with growth plate closure of the tibia, resulting in a reduced overall tibial length. In later years, Howe and Webster reported large areas of calcification in the nasal septum in the same rat model, resulting in maxillonasal hypoplasia and reduction in the length of the nasal bones [8]. These features resembled warfarin embryopathy (the fetal warfarin syndrome) in humans, resulting from the use of warfarin during the first trimester of pregnancy (see paragraph 2.6). It was only after the identification of MGP in cartilage, that it was hypothesized that the cartilage calcification in the warfarin model resulted from loss of MGP function [9].

After its discovery in bone and cartilage, it was thought for many years that the activity of MGP was restricted to these tissues. By targeted deletion of the MGP gene in mice it became clear, however, that MGP also inhibits medial artery calcification. MGP null mice all died within six to eight weeks after birth due to calcification of the elastic lamellae in the tunica media, resulting in rupture of the aorta [10]. The arterial calcifications in the MGP null mice consisted of calcium-phosphate precipitation in a ratio similar to hydroxyapatite, thus mimicking bone mineralization. The warfarin rat model provided further evidence that impairment of MGP function results in vascular calcification [11]. Price et al. investigated the presence of vascular calcification in this model and reported that aortic calcification was detectable after only two weeks of warfarin treatment. The calcification was localized at the elastic fibres within the tunica media, similar as seen in MGP null mice.

A mechanism explaining the strong calcification inhibitory activity of MGP was first suggested by Price, which was that MGP binds tightly to crystal nuclei, thereby preventing further growth [11]. Vascular calcification is currently considered to be an active, cellularmediated process, in which the differentiation of VSMC from a contractile phenotype into a synthetic phenotype with chondrocyte- and osteoblast-like cell features, plays an important role (see 3. Arterial calcification) [12]. A second mechanism of MGP action is thought to be direct inhibition of VSMC differentiation, which is supported by the findings in MGP null mice. VSMC of these mice were changed into chondrocyte-like cells at sites of calcification, with the appearance of cartilage nodules in the calcified aorta at the age of one month [10,13]. In addition, a loss of smooth muscle markers and upregulated expression of the bone-specific transcription factor cbf1a/Runx2 and the osteogenic protein osteopontin was found in MGP null mice [14]. 


\section{General Introduction}

The ability of MGP to keep VSMC in the contractile phenotype may be accomplished by its binding to bone morphogenetic protein-2 (BMP-2) $[15,16]$. BMP-2 is a member of the transforming growth factor- $\beta$ (TGF- $\beta$ ) superfamily, and is an osteogenic growth factor. BMP-2 has been shown to be expressed in human atherosclerotic lesions [17]. Wallin et al. demonstrated that only CMGP species bind to BMP-2 [15], thereby likely blocking the osteo-inductive properties of BMP-2 [18]. Via its C-terminal region, MGP can also bind to the extracellular glycoprotein vitronectin, which is present in the extracellular matrix of the arteries [19]. The C-terminal part of MGP is hydrophobic and does not contain Glu or Ser residues, which are all present in the more hydrophilic $\mathrm{N}$-terminal and mid-section of the molecule. It may be hypothesized that the binding of MGP to vitronectin results in a concentration of calcification-inhibitory activity in the milieu surrounding the elastic fibers, thereby protecting them from mineralization.

The specific knock-in expression of MGP in VSMC of MGP null mice completely rescued the calcification phenotype [20]. Expression of MGP in the liver of MGP null mice resulted in high levels of circulating MGP. However, the high systemic levels of MGP had no effect on inhibition of arterial calcification. This implies that MGP inhibits calcification by acting within its tissue of synthesis, and not by acting systemically. Evidence for the pivotal role of Gla residues for MGP function was provided by Murshed et al. who generated transgenic mice that produced MGP which could not be carboxylated, since the Glu residues in the Gla-domain were mutated into aspartate residues. Using these mice, it was demonstrated that only cMGP exhibits anti-mineralization properties [20].

In humans, loss-of-function mutations result in the Keutel syndrome [21], a rare autosomal recessive disorder characterized by abnormal cartilage calcification and peripheral pulmonary artery stenoses [22]. Keutel et al. first described the syndrome in two siblings presenting abnormal cartilage calcification, brachytelephalangism, neural hearing loss, and peripheral pulmonary stenosis [23]. At the age of 38, one of the two siblings died of acute right heart failure after several years of obstructive pulmonary disease; postmortem examination revealed calcification of arteries throughout the body resulting in pulmonary artery stenoses and multiple brain infarctions [24]. 


\subsection{MGP in vascular tissue}

Immunohistochemical studies have shown that in healthy vessels MGP is synthesized at relatively low rate $[2,25,26]$, most likely because the need for calcification inhibition is low. However, Shanahan et al. showed that in arteries of diabetic patients lower levels of MGP protein were present than in normal vessels, suggesting that low MGP levels might predispose for calcification [27]. High MGP levels have been detected in arteries with calcification $[2,25,26]$. This may originate from increased MGP synthesis, which has been reported in both medial and intimal arterial calcification $[2,25,26]$, or from accumulation of MGP due to binding to calcium salt crystals.

With the development of species-specific antibodies, enabling the detection of CMGP and ucMGP, it became clear that specifically ucMGP species accumulate in atherosclerotic and calcified arteries $[25,28,29]$. cMGP species were nearly absent in these arteries. Sweatt et al. demonstrated that calcified arterial lesions in aging rats contained elevated levels of ucMGP species, which were not able to bind BMP-2 [28]. Our group demonstrated massive accumulation of UCMGP around calcified lesions which are rapidly formed in the arteries of rats during treatment with warfarin. Additionally, it appeared that high vitamin $\mathrm{K}$ intake resulted in improved MGP carboxylation, regression of pre-formed calcifications and subsequent increased vascular elasticity [30]. The group of Shanahan showed that arteries from children with chronic kidney disease (before the initiation of dialysis), contained more ucMGP species in comparison with vessels from healthy children. This shift preceded the formation of vascular calcification.

\subsection{MGP in the circulation}

MGP is a local inhibitor of vascular calcification and it has been demonstrated that circulating MGP has no biological function [20]. However, circulating MGP may reflect calcification processes and inhibition of those processes in the vascular wall. Circulating levels of MGP will depend on the rate of MGP synthesis in tissue, its secretion from VSMC, and subsequent binding of MGP to calcified areas that may be present within the arterial wall. Mature MGP is highly insoluble and it is currently not known in which forms MGP circulates. It may circulate as a free protein, or in association with a carrier protein. Full length MGP has been purified from the plasma of rats as a complex including calcium, phosphate, carboxylated MGP, and fetuin-A [31]. Additionally, it is likely that also a substantial fraction of circulating MGP occurs as fragments. 


\section{General Introduction}

Two assays to measure circulating MGP species were described in the literature before we started the work described in this thesis. The first assay is a radioimmunoassay (not commercially available), in which polyclonal antibodies directed against MGP purified from human bone are used [32]. It is therefore not possible to discriminate between the different MGP species. Using this assay, O'Donnell et al. found a significant positive correlation between circulating MGP levels and coronary heart disease risk factors (Framingham CHD risk score) in both men and women [33]. Especially the traditional lipid-risk factors correlated significantly with serum MGP levels. However, no significant correlation was found between MGP levels and the extent of coronary artery calcification (CAC).

The second assay is an enzyme-linked immunosorbent assay (ELISA). With the development of species-specific antibodies by our group, it became possible to construct MGP ELISA for different MGP species (Figure 3). Two types of ELISA were developed: monoantibody (competitive) and dual-antibody (sandwich) ELISA. The first MGP ELISA was described by our group in 2000, and is now commercially available as an ELISA kit produced by Biomedia (Austria, Vienna) [34, 35]. This is a mono-antibody ELISA, in which a monoclonal antibody against the non-phosphorylated N-terminal MGP amino-acid sequence 315 is used. This assay measures dPMGP species, and does not discriminate between cMGP and ucMGP. Therefore, the MGP measurements with this assay are referred to as measurements of total dpMGP ( $t$-dpMGP) in this thesis (Figure 3). Increased levels of serum $t$ dpMGP were measured in patients with severe atherosclerosis and with type I diabetes mellitus [34]. The same assay was used in collaboration with Jono and colleagues, in which the severity of CAC was measured with electron beam computed tomography in subjects with suspected coronary artery disease [36]. The serum levels of t-dpMGP were significantly lower in subjects with CAC compared to those without CAC. Moreover, tdpMGP levels inversely correlated with the severity of CAC.

Since 2000, we developed three new MGP ELISA: a mono-antibody assay measuring total uncarboxylated MGP species (t-ucMGP) and two dual-antibody assays measuring desphosphorylated-uncarboxylated MGP (dp-ucMGP) and desphosphorylatedcarboxylated (dp-cMGP) species, respectively. The characteristics of these assays as well as their application in several patient populations are described in this thesis. 


\section{Vitamin $\mathrm{K}$}

\subsection{Discovery}

Vitamin K was discovered in the early 1930s by the Danish biochemist Henrik Dam. During his research on cholesterol metabolism in chickens, Dam observed that chicks reared on a diet free of sterols and poor in fat developed large subcutaneous and intramuscular hemorrhages [37]. Additionally, it was reported by McFarlane et al. that blood of chicks fed an ether-extracted fish or meat meal failed to coagulate overnight [38]. Initial experiments with additions of lemon juice, cholesterol, cod-liver oil, or vitamin C to the diet failed to prevent these haemorrhages [37]. Further testing of different foods, ranging from cereals and seeds to animal organs, revealed that "the antihemorraghic factor" was fat-soluble, with one of its richest sources being hog-liver [39]. Hendrik Dam designated this factor as Koagulations vitamin (abbreviated as vitamin $\mathrm{K}$ ), because of its requirement for normal haemostasis [39]. Subsequently, Almquist and Stokstad reported that the haemorrhagic syndrome could be prevented by concentrated extracts of alfalfa as well as by fish meals and bran preparations, which were subjected to the action of microorganisms [40].

In the mid 1930s, Schønheyder proposed that the prolonged clotting time seen in animals with the hemorrhagic syndrome was due to a decreased concentration of plasma prothrombin [41]; indeed, precipitates of prothrombin from the plasma of " $\mathrm{K}$ avitaminous" chicks were found to be inactive [42]. Additionally, the prothrombin time of chicks and rabbits fed with spoiled sweet clover hay - resulting in hemorrhagic disease was increased $[43,44]$. In later years, it was established that sweet clover hay contained dicoumarin, a vitamin $\mathrm{K}$ antagonist produced by moulds acting on the hay [45].

In the late 1930s, several research groups were able to isolate vitamin K from alfalfa as a yellow oil [46-49]. The group at St. Louis University led by Edward A. Doisy could also isolate vitamin $\mathrm{K}$ from putrefied fish meals as a crystalline product and designated this product as vitamin $\mathrm{K}_{2}[50,51]$. During this time, the first clinical studies with administration of vitamin $\mathrm{K}$ to patients with obstructive jaundice or biliary problems were conducted [52], since it had been recognized that the bleeding tendency seen in these cases was related to vitamin $\mathrm{K}$ deficiency [53]. Additionally, vitamin $\mathrm{K}$ was administered to newborns suffering from haemorrhagic disease or who were found to have a prolonged prothrombin time [54]. In later years, the prophylactic treatment of newborns (and/or their mothers) with vitamin K gained increasing attention [55]. Dam mentioned in his Nobel lecture that prophylactic treatment with vitamin $\mathrm{K}$ decreased the neonatal mortality rate among newborn infants from $4.6 \%$ to $1.8 \%[56,57]$. Dam and Doisy received the 1943 Nobel prize in Physiology/Medicine for the discovery of vitamin K and its chemical nature [56]. 


\subsection{Function}

Until 1974, various hypotheses about the mechanism of vitamin $\mathrm{K}$ action were proposed, including a role in mitochondrial electron transport and induction of protein synthesis. The discovery of the unusual amino acid $\nu$-carboxyglutamate acid (Gla) in prothrombin as the product of vitamin $\mathrm{K}$ action in 1974, finally identified the unequivocal role of vitamin $\mathrm{K}$ as a cofactor for the post-translational carboxylation of glutamate (Glu) residues [58, 59] (Figure 2). These findings triggered the development of an in vitro carboxylase assay by the group of Suttie, in which ${ }^{14} \mathrm{CO}_{2}$ was incorporated into Glu-containing synthetic peptides [60]. Thus, it was demonstrated that the vitamin K-dependent step in prothrombin synthesis involved the Y-carboxylation of Glu residues in precursor prothrombin [60]. Fifteen years later, Stafford and co-workers succeeded in purifying the key enzyme GGCX [61], which carries out the carboxylation reaction. Only recently the purification and amino acid sequence of the second key enzyme in vitamin $\mathrm{K}$ metabolism, vitamin $\mathrm{K}$ epoxide reductase (VKOR), were reported by two independent groups $[62,63]$.

\subsection{K vitamins}

Vitamin $\mathrm{K}$ can be considered a group name for different chemical forms of vitamin $\mathrm{K}$ (K vitamins). All forms possess a common 2-methyl-1,4-napthoquinone structure (menadione) and an aliphatic side-chain at the 3-position (Figure 4) [64]. The major dietary source of vitamin $\mathrm{K}$ is phylloquinone (vitamin $\mathrm{K}_{1}$ ), originating from green leafy plants and green vegetables. The chemical structure of vitamin $K_{2}$ was elucidated in the 1960 s and revealed that it consists of a family of related products now known as menaquinones [65]. Menaquinones are mainly of microbial origin and their nomenclature is based on the number of isoprenoid residues in their side-chain (Figure 4). Dietary relevant menaquinones range from menaquinone-4 (MK-4) through menaquinone-10 (MK-10). In the human diet the main sources of menaquinones are cheese, curd, and the Japanese food natto. MK-4 is the only menaquinone that can be produced in mammalian tissues, where it is formed by conversion of phylloquinone and possibly other menaquinones [64, 66]. Vitamin K1, MK-4, and MK-7 are commercially available products. 


\section{Figure 4: $\mathrm{K}$ vitamins}<smiles>CC1=CC(=O)c2ccccc2C1=O</smiles>

Vitamin $\mathrm{K}_{3}$ (menadione)<smiles>CC(C)=CCC1=C(C)C(=O)c2ccccc2C1=O</smiles>

Vitamin $\mathrm{K}_{1}$ (phylloquinone)<smiles>CC(C)=CCCC(C)=CCC1=C(C)C(=O)c2ccccc2C1=O</smiles>

Vitamin $\mathrm{K}_{2}$ (menaquinone)

Chemical structure of vitamin $K_{3}$ (menadione), $K_{1}$ (phylloquinone), and vitamin $K_{2}$ (menaquinone). The (n) stands for number of isoprenoid residues. 


\subsection{Vitamin K-dependent proteins (Gla-proteins)}

After the discovery of Gla residues in prothrombin, the subsequently identified proteins containing these residues were those involved in blood coagulation: factors $\mathrm{VII}, \mathrm{IX}$, and $\mathrm{X}$ as well as the proteins C, S, and Z [67]. During this time, the presence of carboxylase was demonstrated in a wide variety of non-hepatic tissues, ranging from bone to skin [68, 69]. It was assumed that the endogenous substrate of carboxylase in these tissues (unidentified Gla-proteins) was not involved in blood coagulation.

The first extra-hepatic sites in which Gla-proteins have been identified and characterized are bone and cartilage. Bone Gla-protein, also known as osteocalcin, was identified in the mid 1970s [70, 71] and presently serves as a marker for osteoblastic activity. MGP was identified in the early 1980s [1]. Since then, several new Gla-proteins have been identified, including growth-arrest-specific gene 6 protein (Gas-6) [72]. Gas-6, which plays a role in cell growth and apoptosis, has gained increasing attention in the past years. The most recently indentified Gla-protein is Gla-Rich protein (GRP), which is thought to serve as an inhibitor of soft tissue calcification [73]. An overview of all known Gla-proteins is given in Table 2.

Table 2: Gla-proteins

\begin{tabular}{|c|c|c|}
\hline $\begin{array}{l}\text { Gla-protein } \\
\text { Hepatic [74-76] }\end{array}$ & Function & Site of action \\
\hline FII (prothrombin), FVII, FIX, FX & Procoagulants & Plasma \\
\hline Protein C, Z & Anticoagulants & Plasma \\
\hline Protein S & Anticoagulant role as cofactor for protein $\mathrm{C}$ & $\begin{array}{l}\text { Plasma and variety of other tissues } \\
\text { (endothelium, bone) }\end{array}$ \\
\hline \multicolumn{3}{|l|}{ Extra-hepatic } \\
\hline $\begin{array}{l}\text { Osteocalcin (OC, bone gla-protein) } \\
{[70,77]}\end{array}$ & Regulator of orderly bone crystallization & Bone, dentin \\
\hline Matrix Gla protein (MGP) [1] & Inhibitor of ectopic calcification & $\begin{array}{l}\text { Cartilage and soft tissues including } \\
\text { vasculature }\end{array}$ \\
\hline $\begin{array}{l}\text { Growth arrest specific gene- } 6 \\
\text { protein (Gas-6) [78] }\end{array}$ & Regulator of cell growth & Soft tissues \\
\hline Gla-rich protein (GRP) [73] & $\begin{array}{l}\text { Regulator of calcium availability in the } \\
\text { extra-cellular matrix }\end{array}$ & Cartilage, bone, and soft tissues \\
\hline \multicolumn{3}{|l|}{ Membrane-bound } \\
\hline Carboxylase [79] & Enzyme for carboxylation & Soft tissues \\
\hline Proline rich Gla-protein (PRGP) [80] & Potentially promotes signal transduction & Soft tissues \\
\hline $\begin{array}{l}\text { Transmembrane Gla-proteins (TMG) } \\
\text { [81] }\end{array}$ & Unknown & Soft tissues \\
\hline \multicolumn{3}{|l|}{ Extra-cellular matrix } \\
\hline $\begin{array}{l}\text { Periostin (osteoblast specific factor- } \\
\text { 2) [82] }\end{array}$ & $\begin{array}{l}\text { Promotes cell adhesion, motility, migration, } \\
\text { and angiogenesis }\end{array}$ & Remodelling tissues, bone (periosteum) \\
\hline TGF- $\beta$ induced gene h3 ( $\beta$ ig-h3) [82] & $\begin{array}{l}\text { Promotes cell migration and cell-matrix } \\
\text { interaction }\end{array}$ & Soft tissues, bone, cartilage \\
\hline
\end{tabular}

Abbreviations used: $F$, factor; TGF- $\beta$, transforming growth factor-beta. 


\subsection{Dietary intake}

As compared with other vitamins, the dietary intake of vitamin $\mathrm{K}$ is very low. The recommended dietary allowance (RDA) is $1 \mu \mathrm{g} / \mathrm{kg} /$ day. Also, its biological half life is relatively short: upon deprivation, experimental animals develop symptoms of vitamin $\mathrm{K}$ deficiency within a few days. Yet, vitamin $\mathrm{K}$ intake is sufficient to ensure normal haemostasis in healthy adults. It should be kept in mind, however, that the liver - which is the place of coagulation factor synthesis - is capable of extracting vitamin $\mathrm{K}$ form the circulation very efficiently. It is questionable whether the present RDA for vitamin $\mathrm{K}$ intake is sufficient to cover the requirements of extrahepatic tissues, including the vascular wall. Increased availability of vitamin $\mathrm{K}$ could therefore have a beneficial effect on vascular health.

The association between dietary vitamin $\mathrm{K}_{1}$ and $\mathrm{K}_{2}$ intake and cardiovascular mortality and calcification has been investigated in several Dutch population-based studies. In the Rotterdam Study, dietary vitamin $\mathrm{K}_{2}$ intake (and not vitamin $\mathrm{K}_{1}$ ) was inversely correlated with cardiovascular calcification and cardiovascular death [83]. Elderly subjects in the highest tertile of vitamin $\mathrm{K}_{2}$ intake had about $50 \%$ reduction in both aortic calcification and cardiovascular mortality, and $25 \%$ decreased all-cause mortality. Among post-menopausal women, participants of the PROSPECT study investigating cancer and nutrition, vitamin $\mathrm{K}_{2}$ intake (and not vitamin $\mathrm{K}_{1}$ ) was associated with decreased coronary calcification $(R R=0.80)$ [84]. Vitamin $\mathrm{K}_{2}$ intake was also inversely associated with CHD risk ( $\mathrm{HR}=0.91$ per $10 \mu \mathrm{g} / \mathrm{day}$ intake), mainly due to the menaquinones MK-7, MK-8, and MK-9 [85]. In a 3-year clinical intervention study in 78 women between 55 and 65 years of age, the effect of vitamin $K_{1}$ supplementation ( $1 \mathrm{mg} /$ day) on vascular characteristics (elasticity and distensibility) was investigated [86]. Vascular elasticity decreased by $10-13 \%$ in the placebo group, but in the vitamin $\mathrm{K}$ group vascular characteristics remained unchanged. This suggested that the process of vascular aging can be retarded by increased vitamin $\mathrm{K}$ intake.

The concept of calcification inhibition by high vitamin $\mathrm{K}$ intake has also been investigated in experimental animals, including the warfarin rat model [87]. In this model, the efficacy of vitamin $\mathrm{K}_{1}$ and MK-4 (vitamin $\mathrm{K}_{2}$ ) in preventing arterial calcification was compared. It was found that MK-4 completely inhibited tissue calcification, whereas a similar or even an 8fold higher dose of vitamin $\mathrm{K}_{1}$ had no measurable effect. The same animal model was used to study the effect of high vitamin $\mathrm{K}$ intake on potential regression of arterial calcification [30]. In this experiment, rats receiving the standard chow (control) had very low aortic calcium during the entire experiment, whereas a 6-week warfarin treatment led to the accrual of calcium salts up to 12 -fold above the baseline values. During the following 6 -week period, warfarin treatment was stopped and the animals received either standard chow or standard food fortified with a high dose of vitamin $\mathrm{K}_{1}$ or $\mathrm{K}_{2}$ (MK-4). 


\section{General Introduction}

It was shown that once calcium deposits had formed, the accrual of calcium salts in the vasculature increased linearly after the warfarin diet had been replaced by the normal dose of vitamin $\mathrm{K}$. At high doses of either $\mathrm{K}_{1}$ or $\mathrm{K}_{2}$, however, the process of calcification was not only stopped, but a significant fraction (some $40 \%$ ) of the previously formed calcium salts had been removed within 6 weeks. This effect was found both in the aorta and in the coronary arteries. Using immunohistochemical staining with the species-specific MGP antibodies, it was demonstrated that parallel to the regression of aortic calcium content, cMGP had increased and UcMGP had decreased. This suggests a role of activated MGP in the regression of calcified plaques.

\subsection{Vitamin $\mathrm{K}$ antagonists}

Vitamin $\mathrm{K}$ antagonists (also known as oral anticoagulants, OAC) block the recycling of vitamin $\mathrm{K}$ by inhibition of the enzyme VKOR, resulting in vitamin K deficiency. Treatment with vitamin $\mathrm{K}$ antagonists will therefore result in the synthesis of uncarboxylated vitamin $\mathrm{K}$ dependent proteins. VKOR is a crucial enzyme in vitamin $\mathrm{K}$ metabolism and ensures the reutilization of vitamin $\mathrm{K}$ after it has been oxidized in the carboxylase reaction. Because of this recycling, human vitamin $\mathrm{K}$ requirement is low [88]. On a molecular level, VKOR reduces vitamin $\mathrm{K}$ epoxide (KO) in two steps: first to vitamin $\mathrm{K}$ quinone $(\mathrm{K})$, and subsequently to vitamin $\mathrm{K}$ hydroquinone $\left(\mathrm{KH}_{2}\right)$, the latter being the active cofactor for GGCX (Figure 2). The cytoplasmic enzyme DT-diaphorase serves as a rescue enzyme when coumarins are used; it is capable of reducing vitamin $\mathrm{K}$ quinones to their hydroquinone forms. However, Wallin et al. showed that the enzyme DT-diaphorase in VSMC is 100 -fold less active than in the liver [89]. Coumarin treatment may therefore have a detrimental effect in the arterial vessel wall, by blocking vitamin K-metabolism, which may impair MGP activity

It has been known for many years, that women receiving the vitamin Kantagonist warfarin during the first trimester of pregnancy are at risk of delivering children with a syndrome characterized by nasal hypoplasia, depression of the nasal bridge, and punctuate calcifications in the axial skeleton, proximal femurs, and calcanei [90]. This syndrome is known as warfarin embryopathy (fetal warfarin syndrome), and the abnormalities were first believed to result from calcified haemorrhages in the developing fetal cartilages [90-92]. However, this was unlikely since fetal clotting factors are absent during the first trimester of pregnancy $[90,93]$. Similarities between the facial and skeletal abnormalities seen in warfarin embryopathy and the fetal phenytoin (hydantoin) syndrome suggest that prenatal vitamin K-deficiency may underlie these abnormalities [90, 94]. This was confirmed by Pauli et al. who described a congenital deficiency of the VKOR enzyme, causing prenatal vitamin $\mathrm{K}$ deficiency, resulting in a similar phenotype $[95,96]$. 
In later years, it was reported that also dietary vitamin $\mathrm{K}$ deficiency results in comparable calcification abnormalities [97-101], which were remarkably similar to the bone and cartilage defects observed in warfarin-treated rats [7].

Vitamin $\mathrm{K}$ antagonists are frequently used to prevent thrombosis in high-risk patients [102]. Treatment periods range from several weeks to many years, often even life-long [103]. After demonstration in animal models that vitamin $\mathrm{K}$ antagonists induce vascular calcification, several independent studies showed an association between the use of coumarins and arterial and/or valvular calcification. Schurgers et al. compared the extent of aortic valve calcification in patients receiving marcoumar treatment (mean duration 25 months) with patients not on OAC before aortic valve replacement surgery [104]. The mean calcified area was $16 \%$ in the non-treated group and $37 \%$ in the marcoumar group. Histopathological evaluation showed partial or total valve destruction induced by amorphous calcified deposits in the latter group. These data were confirmed by Koos et al., who showed that patients on long term OAC treatment had significantly higher aortic valve and coronary artery calcification scores compared patients without anticoagulation treatment, as assessed with MSCT scanning [105]. Rennenberg et al. compared 19 patients who used coumarins for more than 10 years with 18 matched healthy controls [106]. Vascular calcification was quantified using a plain X-ray of the thighs, from which femoral arterial calcifications could be assessed. The odds ratio for calcification in patients versus controls was 8.5. Coumarin use was independently associated with calcification. Since coumarin derivatives are widely used, physicians should be aware of this potential adverse effect on the vasculature. 


\section{Arterial calcification}

Until a decade ago, vascular calcification was thought to be a passive, clinically irrelevant process, resulting from a high calcium-phosphate product, inflammation, lipid accumulation, and/or diabetes mellitus. However, during recent years it has become increasingly clear that vascular calcification is an active, cell-mediated process and an important, independent pathology that is strongly associated with increased risk of cardiovascular morbidity and mortality [107-109]. Four different types of cardiovascular calcification can be distinguished: atherosclerotic calcification, medial artery calcification, cardiac valve calcification, and calciphylaxis [110]. Atherosclerotic or intimal calcification is seen at sites of atherosclerotic plaques, in which cellular necrosis, inflammation, and cholesterol deposition occur. The presence of this type of calcification is associated with atherosclerotic burden. Medial artery calcification occurs in the elastic lamina of large- and medium- to small-sized arteries. It is commonly seen in CKD, diabetes, and aging. On radiographs, this type of calcification can be seen in distal arteries as a "railroad track" pattern. Whereas intimal calcification is mainly associated with arterial obstruction, medial calcification is associated with vascular stiffness. In cardiac valve calcification aspects of both medial and atherosclerotic calcification can be seen, with the primary stimulus being a combination of mechanical stress and inflammation [110]. Calciphylaxis (calcific uremic arteriolopathy) is characterized by medial calcification with intimal proliferation of small- to mediumsized arteries, resulting in tissue necrosis. The various types and localizations of vascular calcifications have an impact on cardiac morbidity mortality not only by increasing and complicating coronary atherosclerosis, but also by increasing the stiffness of the main arteries [111].

The phenotypic change of VSMC from a contractile phenotype into an osteo/chondrocytic phenotype plays a central role in the calcification process [12]. This transformation is characterized by the upregulation of bone-specific transcription factors and matrix proteins, resulting in an expression of osteogenic markers by VMSC [27]. In addition, a mineral imbalance, for instance hypercalcemia and/or hyperphosphatemia, can induce the formation of vesicles by the VSMC. These vesicles can be derived from apoptotic and viable VSMC and - if not phagocytosed properly - form a initial nidus for the deposition of hydroxyapatite crystals [112]. In vitro studies have shown that under physiological circumstances, these vesicles are loaded with mineralization inhibitors, including MGP [112], limiting their mineralization potential. A loss of inhibitors results in vesicles with mineralizing properties [112]. It is therefore thought that a loss of inhibitors, including MGP, is a major factor in inducing calcification [12]. 
Clinically, vascular calcification, particularly medial calcification, causes stiffening of the vascular wall, which may result in decreased arterial compliance, development of left ventricular hypertrophy and decreased coronary perfusion leading to an increased risk of fatal complications $[113,114]$. Calcification is common in the elderly population, and in patients suffering from diseases, such as chronic kidney disease (CKD), diabetes mellitus, aortic stenosis, and atherosclerosis [115]. Therefore, a lot of efforts have been directed towards retarding or reversing the development of calcification in the vasculature. In animal models it has been shown that arterial calcification is reversible [30, 116-118], demonstrating that also the regression process is an actively regulated process.

Patients with CKD, especially those patients receiving hemodialysis or peritoneal dialysis therapy, have the highest incidence of arterial calcification, and cardiovascular mortality is 20 -fold higher than in the apparently healthy population $[119,120]$. Moreover, moderate to severe vascular calcifications are found in 60 to $80 \%$ of patients on hemodialysis [121, 122]. Booth and co-workers demonstrated that vitamin $\mathrm{K}$ status in CKD patients is low [123]. Some $30 \%$ of the hemodialysis patients had sub-clinical vitamin K deficiency, based on measurements of circulating vitamin $\mathrm{K}$ levels. It has been hypothesized that vitamin $\mathrm{K}$ may be a novel treatment option, or at least a modifiable risk factor for vascular calcification in dialysis patients [124]. 


\section{Outline of this thesis}

This thesis focuses on measurements of circulating MGP species and their potential diagnostic and clinical value. Chapter 1 gives an introduction of MGP and vitamin K. In Chapter 2, the mono-antibody MGP ELISA measuring total uncarboxylated MGP (t-ucMGP) levels is presented and characterised, with application of t-ucMGP measurements in patient populations with cardiovascular disease (coronary angioplasty and aortic valve stenosis) and vascular calcification (hemodialysis and calciphylaxis). Further investigation of t-ucMGP as a biomarker in dialysis patients is described in Chapters 3 and 4. Chapter 3 describes the correlation between t-ucMGP levels and the extent of coronary artery calcification in hemodialysis patients. In Chapter 4, the association between t-ucMGP levels and (cardiovascular) mortality in a large Dutch cohort of hemodialysis and peritoneal dialysis patients is investigated. In Chapter 5, we compared the four available MGP assays, including two novel dual-antibody MGP ELISA. All assays were applied in patients with cardiovascular disease, rheumatic diseases, and end-stage renal disease. To determine which assay would be suited to assess vascular vitamin $\mathrm{K}$ status, the effect of changes in vitamin $\mathrm{K}$ status on circulating MGP species was also investigated. For this, the assays were applied in two groups of healthy subjects with opposite vitamin $\mathrm{K}$ status: subjects receiving vitamin $\mathrm{K}$ supplementation and those receiving vitamin $\mathrm{K}$ antagonists. In Chapter 6, we investigated the functions of MGP in humans by phenotypic characterization of a newly-identified Dutch patient with Keutel syndrome. In order to further elucidate the difference in phenotype between MGP deficient mice and men, we characterized the circulating MGP species of this patient and his firstdegree relatives. Additionally, MGP species were determined in tissues from the first described Keutel syndrome patient in the literature. Chapter $\mathbf{7}$ describes the investigation of the vitamin $\mathrm{K}$ intake and the hepatic and extra-hepatic vitamin K status in a Dutch cohort of hemodialysis patients. 


\section{References}

1. Price, P.A., M.R. Urist, and Y. Otawara, Matrix Gla protein, a new gamma-carboxyglutamic acid-containing protein which is associated with the organic matrix of bone. Biochem Biophys Res Commun, 1983. 117(3): p. 765-71.

2. Shanahan, C.M., et al., High expression of genes for calcification-regulating proteins in human atherosclerotic plaques. J Clin Invest, 1994. 93(6): p. 2393-402.

3. Fraser, J.D. and P.A. Price, Lung, heart, and kidney express high levels of mRNA for the vitamin K-dependent matrix Gla protein. Implications for the possible functions of matrix Gla protein and for the tissue distribution of the gamma-carboxylase. J Biol Chem, 1988. 263(23): p. 11033-6.

4. Berkner, K.L., The vitamin K-dependent carboxylase. J Nutr, 2000. 130(8): p. 1877-80.

5. Price, P.A., J.S. Rice, and M.K. Williamson, Conserved phosphorylation of serines in the Ser-X-Glu/Ser(P) sequences of the vitamin $K$-dependent matrix Gla protein from shark, lamb, rat, cow, and human. Protein Sci, 1994. 3(5): p. 822-30.

6. Wajih, N., et al., Processing and transport of matrix gamma-carboxyglutamic acid protein and bone morphogenetic protein-2 in cultured human vascular smooth muscle cells: evidence for an uptake mechanism for serum fetuin. J Biol Chem, 2004. 279(41): p. 43052-60.

7. Price, P.A., et al., Excessive mineralization with growth plate closure in rats on chronic warfarin treatment. Proc Natl Acad Sci U S A, 1982. 79(24): p. 7734-8.

8. Howe, A.M. and W.S. Webster, The warfarin embryopathy: a rat model showing maxillonasal hypoplasia and other skeletal disturbances. Teratology, 1992. 46(4): p. 379-90.

9. Hale, J.E., J.D. Fraser, and P.A. Price, The identification of matrix Gla protein in cartilage. J Biol Chem, 1988. 263(12): p. 5820-4.

10. Luo, G., et al., Spontaneous calcification of arteries and cartilage in mice lacking matrix GLA protein. Nature, 1997. 386(6620): p. 78-81.

11. Price, P.A., S.A. Faus, and M.K. Williamson, Warfarin causes rapid calcification of the elastic lamellae in rat arteries and heart valves. Arterioscler Thromb Vasc Biol, 1998. 18(9): p. 1400-7.

12. Shanahan, C.M., Vascular calcification--a matter of damage limitation? Nephrol Dial Transplant, 2006. 21(5): p. 1166-9.

13. El-Maadawy, S., et al., Cartilage formation and calcification in arteries of mice lacking matrix Gla protein. Connect Tissue Res, 2003. 44 Suppl 1: p. 272-8.

14. Steitz, S.S.A., et al., Smooth muscle cell phenotypic transition associated with calcification: upregulation of Cbfa1 and downregulation of smooth muscle lineage markers. Circulation research, 2001. 89(12): p. 1147-54.

15. Wallin, R., et al., Modulation of the binding of matrix Gla protein (MGP) to bone morphogenetic protein-2 (BMP-2). Thromb Haemost, 2000. 84(6): p. 1039-44.

16. Zebboudj, A.F., M. Imura, and K. Bostrom, Matrix GLA protein, a regulatory protein for bone morphogenetic protein-2. J Biol Chem, 2002. 277(6): p. 4388-94

17. Boström, K., et al., Bone morphogenetic protein expression in human atherosclerotic lesions. J Clin Invest, 1993. 91(4): p. 1800-9.

18. Bostrom, K., et al., Matrix GLA protein modulates differentiation induced by bone morphogenetic protein-2 in C3H10T1/2 cells. J Biol Chem, 2001. 276(17): p. 14044-52.

19. Nishimoto, S.K. and M. Nishimoto, Matrix Gla protein C-terminal region binds to vitronectin. Co-localization suggests binding occurs during tissue development. Matrix Biol, 2005. 24(5): p. 353-61.

20. Murshed, M., et al., Extracellular matrix mineralization is regulated locally; different roles of two glacontaining proteins. J Cell Biol, 2004. 165(5): p. 625-30.

21. Munroe, P.B., et al., Mutations in the gene encoding the human matrix Gla protein cause Keutel syndrome. Nat Genet, 1999. 21(1): p. 142-4.

22. Hur, D.J., et al., A novel MGP mutation in a consanguineous family: review of the clinical and molecular characteristics of Keutel syndrome. Am J Med Genet A, 2005. 135(1): p. 36-40.

23. Keutel, J., G. Jorgensen, and P. Gabriel, A new autosomal-recessive hereditary syndrome. Multiple peripheral pulmonary stenosis, brachytelephalangia, inner-ear deafness, ossification or calcification of cartilages. Dtsch Med Wochenschr, 1971. 96(43): p. 1676-81 passim. 
24. Meier, M., et al., Tracheobronchial stenosis in Keutel syndrome. Eur Respir J, 2001. 17(3): p. 566-9.

25. Schurgers, L.J., et al., Novel conformation-specific antibodies against matrix gamma-carboxyglutamic acid (Gla) protein: undercarboxylated matrix Gla protein as marker for vascular calcification. Arterioscler Thromb Vasc Biol, 2005. 25(8): p. 1629-33.

26. Dhore, C.R., et al., Differential expression of bone matrix regulatory proteins in human atherosclerotic plaques. Arterioscler Thromb Vasc Biol, 2001. 21(12): p. 1998-2003.

27. Shanahan, C.M., et al., Medial localization of mineralization-regulating proteins in association with Monckeberg's sclerosis: evidence for smooth muscle cell-mediated vascular calcification. Circulation, 1999. 100(21): p. 2168-76.

28. Sweatt, A., et al., Matrix Gla protein (MGP) and bone morphogenetic protein-2 in aortic calcified lesions of aging rats. J Thromb Haemost, 2003. 1(1): p. 178-85.

29. Gheduzzi, D., et al., Matrix Gla protein is involved in elastic fiber calcification in the dermis of pseudoxanthoma elasticum patients. Lab Invest, 2007. 87(10): p. 998-1008.

30. Schurgers, L.J., et al., Regression of warfarin-induced medial elastocalcinosis by high intake of vitamin $K$ in rats. Blood, 2007. 109(7): p. 2823-31.

31. Price, P.A., et al., Discovery of a high molecular weight complex of calcium, phosphate, fetuin, and matrix gamma-carboxyglutamic acid protein in the serum of etidronate-treated rats. J Biol Chem, 2002. 277(6): $p$. 3926-34.

32. Otawara, Y.Y. and P.P.A. Price, Developmental appearance of matrix GLA protein during calcification in the rat. J Biol Chem, 1986. 261(23): p. 10828-32.

33. O'Donnell, C.J., et al., Matrix Gla protein is associated with risk factors for atherosclerosis but not with coronary artery calcification. Arterioscler Thromb Vasc Biol, 2006. 26(12): p. 2769-74.

34. Braam, L.A., et al., Assay for human matrix gla protein in serum: potential applications in the cardiovascular field. Arterioscler Thromb Vasc Biol, 2000. 20(5): p. 1257-61.

35. Schurgers, L.J., et al., Characteristics and performance of an immunosorbent assay for human matrix Glaprotein. Clin Chim Acta, 2005. 351(1-2): p. 131-8.

36. Jono, S., et al., Matrix Gla protein is associated with coronary artery calcification as assessed by electronbeam computed tomography. Thromb Haemost, 2004. 91(4): p. 790-4.

37. Dam, H. and F. Schonheyder, A deficiency disease in chicks resembling scurvy. Biochem J, 1934. 28(4): p. 1355-9.

38. McFarlane, W.D., W.R. Graham, and F.F. Richardson, The fat-soluble vitamin requirements of the chick: The vitamin A and vitamin D content of fish meal and meat meal. Biochem J, 1931. 25(1): p. 358-66.

39. Dam, H., The antihaemorrhagic vitamin of the chick. Biochem J, 1935. 29(6): p. 1273-85.

40. Almquist, H.J. and E.L.R. Stokstad, Hemorrhagic chick disease of dietary origin. J Biol Chem, 1935. 111: p. 105-13.

41. Schønheyder, F.F., The quantitative determination of vitamin K. I. Biochem J, 1936. 30(5): p. 890-6.

42. Dam, H.H., F.F. Schønheyder, and E.E. Tage-Hansen, Studies on the mode of action of vitamin K. Biochem J, 1936. 30(6): p. 1075-9.

43. Quick, A.J., The coagulation defect in sweet clover disease and in the hemorrhagic chick disease of dietary origin: A consideration of the source of prothrombin. Am J Physiol, 1937. 118: p. 260-71.

44. Quick, A.A.J., The development and use of the prothrombin tests. Circulation, 1959. 19(1): p. 92-6.

45. Campbell, H.A. and K.P. Link, Studies on the hemorrhagic sweet clover disease. IV.The isolation and crystallization of the antihemorrhagic agent. J Biol Chem, 1940. 138: p. 21-33.

46. Almquist, H.J., Purification of the antihemorrhagic vitamin. J Biol Chem, 1936. 114: p. 241-5.

47. Almquist, H.J., Purification of the antihemorrhagic vitamin by distillation. J Biol Chem, 1936. 115: p. 589-91.

48. Dam, H., et al., Isoliering des Vitamins $K$ in hochgereinigter Form. Helv Chim Acta, 1939. 22: p. 310-3.

49. Binkley, S.B., et al., The isolation of Vitamin K1. J Biol Chem, 1939. 130: p. 219-34.

50. McKee, R.W., et al., The isolations of vitamins K1 and K2. J Am Chem Soc, 1939. 61: p. 1295.

51. Binkley, S.B., et al., Derivatives of vitamins K1 and K2. J Am Chem Soc, 1939. 61: p.1612-3.

52. Suttie JW. Vitamin K. In: Handbook of lipid research: The fat-soluble vitamins, $1^{\text {st }}$ ed. Plenum Press, $1978:$ p. 211-77. 
53. Dam, H. and J. Glavind, Vitamin K in human pathology. Lancet, 1938. 231: p.720-1.

54. Waddell, W.W. and D. Guerry, The role of vitamin $K$ in the etiology, prevention, and treatment of hemorrhage in the newborn infant. Part II. J Ped, 1939. 15: p. 802-1.

55. Lehmann, J., Vitamin $K$ as a prophylactic in 13.000 infants. The lancet, 1944. 243: p. 493-4.

56. Zetterstrom, R., H. C. P. Dam (1895-1976) and E. A. Doisy (1893-1986): the discovery of antihaemorrhagic vitamin and its impact on neonatal health. Acta Paediatr, 2006. 95(6): p. 642-4.

57. Dam H. The discovery of vitamin K, its biological functions and therapeutical application. Nobel Lecture, December 12, 1946.

58. Stenflo, J., et al., Vitamin $K$ dependent modifications of glutamic acid residues in prothrombin. Proc Natl Acad Sci USA, 1974. 71(7): p. 2730-3.

59. Nelsestuen, G.L., T.H. Zytkovicz, and J.B. Howard, The mode of action of vitamin K. Identification of gammacarboxyglutamic acid as a component of prothrombin. J Biol Chem, 1974. 249(19): p. 6347-50.

60. Esmon, C.T., J.A. Sadowski, and J.W. Suttie, A new carboxylation reaction. The vitamin K-dependent incorporation of H-14-CO3- into prothrombin. J Biol Chem, 1975. 250(12): p. 4744-8.

61. Wu, S.M., D.P. Morris, and D.W. Stafford, Identification and purification to near homogeneity of the vitamin K-dependent carboxylase. Proc Natl Acad Sci USA, 1991. 88(6): p. 2236-40.

62. Mazzini, M.J. and P.C. Schulze, Proatherogenic pathways leading to vascular calcification. Eur J Radiol, 2006. 57(3): p. 384-9.

63. Ludwig, R.J., et al., Psoriasis: a possible risk factor for development of coronary artery calcification. $\mathrm{Br} \mathrm{J}$ Dermatol, 2007. 156(2): p. 271-6.

64. Shearer, M.J. and P. Newman, Metabolism and cell biology of vitamin K. Thromb Haemost, 2008. 100(4): p. 530-47.

65. Jacobsen, B.K. and H. Dam, Vitamin K in bacteria Biochim Biophys Acta, 1960. 40: p. 211-216.

66. Suhara, Y., et al., Structure-activity relationships in the conversion of vitamin $K$ analogues into menaquinone-4. Substrates essential to the synthesis of menaquinone-4 in cultured human cell lines. Bioorg Med Chem. 18(9): p. 3116-24.

67. Suttie, J.W., Vitamin K-dependent carboxylase. Ann Rev Biochem, 1985. 54: p. 459-77.

68. Lian, J.B. and P.A. Friedman, The vitamin K-dependent synthesis of gamma-carboxyglutamic acid by bone microsomes. J Biol Chem, 1978. 253(19): p. 6623-6.

69. de Boer-van den Berg, M.A., C.P. Verstijnen, and C. Vermeer, Vitamin K-dependent carboxylase in skin. J Invest Dermatol, 1986. 87(3): p. 377-80.

70. Hauschka, P.V., J.B. Lian, and P.M. Gallop, Direct identification of the calcium-binding amino acid, gammacarboxyglutamate, in mineralized tissue. Proc Natl Acad Sci USA, 1975. 72(10): p. 3925-29.

71. Price, P.A., et al., Characterization of a gamma-carboxyglutamic acid-containing protein from bone. Proc Natl Acad Sci USA, 1976. 73(5): p. 1447-51.

72. Berkner, K.L., The vitamin K-dependent carboxylase. Annu Rev Nutr, 2005. 25: p. 127-49.

73. Viegas, C.S., et al., Gla-rich protein is a novel vitamin K-dependent protein present in serum that accumulates at sites of pathological calcifications. Am J Pathol, 2009. 175(6): p. 2288-98.

74. Dahlback, B., Blood coagulation. Lancet, 2000. 355(9215): p. 1627-32.

75. Shearer, M.J., Vitamin K metabolism and nutriture. Blood Rev, 1992. 6(2): p. 92-104.

76. Hogg, P.J. and J. Stenflo, Interaction of vitamin K-dependent protein Z with thrombin. Consequences for the amidolytic activity of thrombin and the interaction of thrombin with phospholipid vesicles. J Biol Chem, 1991. 266(17): p. 10953-8.

77. Price, P.A., J.W. Poser, and N. Raman, Primary structure of the gamma-carboxyglutamic acid-containing protein from bovine bone. Proc Natl Acad Sci USA, 1976. 73(10): p. 3374-5.

78. Manfioletti, G., et al., The protein encoded by a growth arrest-specific gene (gas6) is a new member of the vitamin $\mathrm{K}$-dependent proteins related to protein $\mathrm{S}$, a negative coregulator in the blood coagulation cascade. Mol Cell Biol, 1993. 13(8): p. 4976-85.

79. Berkner, K.L. and B.N. Pudota, Vitamin K-dependent carboxylation of the carboxylase. Proc Natl Acad Sci U S A, 1998. 95(2): p. 466-71.

80. Kulman, J.D., et al., Primary structure and tissue distribution of two novel proline-rich gammacarboxyglutamic acid proteins. Proc Natl Acad Sci USA, 1997. 94(17): p. 9058-62. 
81. Kulman, J.D., et al., Identification of two novel transmembrane gamma-carboxyglutamic acid proteins expressed broadly in fetal and adult tissues. Proc Natl Acad Sci USA, 2001. 98(4): p. 1370-5.

82. Coutu, D.L., et al., Periostin, a member of a novel family of vitamin K-dependent proteins, is expressed by mesenchymal stromal cells. J Biol Chem, 2008. 283(26): p. 17991-8001.

83. Geleijnse, J.M., et al., Dietary intake of menaquinone is associated with a reduced risk of coronary heart disease: the Rotterdam Study. J Nutr, 2004. 134(11): p. 3100-5.

84. Beulens, J.W., et al., High dietary menaquinone intake is associated with reduced coronary calcification. Atherosclerosis, 2009. 203(2): p. 489-93.

85. Gast, G.C., et al., A high menaquinone intake reduces the incidence of coronary heart disease. Nutr Metab Cardiovasc Dis, 2009. 19(7): p. 504-10.

86. Braam, L.A., et al., Beneficial effects of vitamins $D$ and $K$ on the elastic properties of the vessel wall in postmenopausal women: a follow-up study. Thromb Haemost, 2004. 91(2): p. 373-80.

87. Spronk, H.M., et al., Tissue-specific utilization of menaquinone-4 results in the prevention of arterial calcification in warfarin-treated rats. J Vasc Res, 2003. 40(6): p. 531-7.

88. Stafford, D.W., The vitamin K cycle. J Thromb Haemost, 2005. 3(8): p. 1873-78.

89. Wallin, R., D. Cain, and D.C. Sane, Matrix Gla protein synthesis and gamma-carboxylation in the aortic vessel wall and proliferating vascular smooth muscle cells--a cell system which resembles the system in bone cells. Thromb Haemost, 1999. 82(6): p. 1764-7.

90. Hall, J.G., R.M. Pauli, and K.M. Wilson, Maternal and fetal sequelae of anticoagulation during pregnancy. Am J Med, 1980. 68(1): p. 122-40.

91. Becker, M.H., et al., Chondrodysplasis punctata: is maternal warfarin therapy a factor? Am J Dis Child 1975. 129(3): p. 356-359.

92. Shaul, W.L., H. Emery, and J.G. Hall, Chondrodysplasia punctata and maternal warfarin use during pregnancy. Am J Dis Child, 1975. 129(3): p. 360-2.

93. Shaul, W.L. and J.G. Hall, Multiple congenital anomalies associated with oral anticoagulants. Am J Obstet Gynecol 1977. 127(2): p. 191-8.

94. Howe, A.M., et al., Prenatal exposure to phenytoin, facial development, and a possible role for vitamin $\mathrm{K}$. Am J Med Genet, 1995. 58(3): p. 238-44.

95. Pauli, R.M., et al., Association of congenital deficiency of multiple vitamin K-dependent coagulation factors and the phenotype of the warfarin embryopathy: clues to the mechanism of teratogenicity of coumarin derivatives. Am J Hum Genet, 1987. 41(4): p. 566-83.

96. Keith, D.A. and P.M. Gallop, Phenytoin, hemorrhage, skeletal defects and vitamin $K$ in the newborn. . Med Hypotheses, 1979. 5: p. 1347-1351.

97. Menger, H., et al., Vitamin $K$ deficiency embryopathy: a phenocopy of the warfarin embryopathy due to a disorder of embryonic vitamin K metabolism. Am J Med Genet, 1997. 72(2): p. 129-34.

98. Khau Van Kien, P., et al., Vitamin K deficiency embryopathy. Am J Med Genet, 1998. 79(1): p. 66-8.

99. Eash, D.D., D.D. Weaver, and N. Brunetti-Pierri, Cervical spine stenosis and possible vitamin $K$ deficiency embryopathy in an unusual case of chondrodysplasia punctata and an updated classification system. Am J Med Genet A, 2003. 122(1): p. 70-5.

100. Jaillet, J., et al., Biliary lithiasis in early pregnancy and abnormal development of facial and distal limb bones (Binder syndrome): a possible role for vitamin K deficiency. Birth Defects Res A Clin Mol Teratol, 2005. 73(3): p. 188-93.

101. Brunetti-Pierri, N., J.V. Hunter, and C.F. Boerkoel, Gray matter heterotopias and brachytelephalangic chondrodysplasia punctata: A complication of hyperemesis gravidarum induced vitamin K deficiency? Am J Med Genet A, 2007. 143(2): p. 200-4.

102. Hirsh, J.J., J.J.F. Cade, and A.A.S. Gallus, Fetal effects of coumadin administered during pregnancy. Blood, 1970. 36(5): p. 623-7.

103. Vermeer, C. and K. Hamulyak, Pathophysiology of vitamin K-deficiency and oral anticoagulants. Thromb Haemost, 1991. 66(1): p. 153-9.

104. Schurgers, L.J., et al., Oral anticoagulant treatment: friend or foe in cardiovascular disease? Blood, 2004. 104(10): p. 3231-2.

105. Koos, R., et al., Relation of oral anticoagulation to cardiac valvular and coronary calcium assessed by multislice spiral computed tomography. Am J Cardiol, 2005. 96(6): p. 747-9. 
106. Rennenberg, R.J., et al., Chronic coumarin treatment is associated with increased extra-coronary arterial calcification in humans. Blood, 2010. 115(24): p. 5121-3.

107. Zieman, S.J., V. Melenovsky, and D.A. Kass, Mechanisms, pathophysiology, and therapy of arterial stiffness. Arterioscler Thromb Vasc Biol, 2005. 25(5): p. 932-43.

108. Doherty, T.M., et al., Calcification in atherosclerosis: bone biology and chronic inflammation at the arterial crossroads. Proc Natl Acad Sci U S A, 2003. 100(20): p. 11201-6.

109. Rosenhek, R., et al., Predictors of outcome in severe, asymptomatic aortic stenosis. N Engl J Med, 2000. 343(9): p. 611-7.

110. Johnson, R.C., J.A. Leopold, and J. Loscalzo, Vascular calcification: pathobiological mechanisms and clinical implications. Circ Res, 2006. 99(10): p. 1044-59.

111. Cannata-Andia, J.B., et al., Vascular calcifications: pathogenesis, management, and impact on clinical outcomes. J Am Soc Nephrol, 2006. 17(12 Suppl 3): p. S267-73.

112. Reynolds, J.L., et al., Human vascular smooth muscle cells undergo vesicle-mediated calcification in response to changes in extracellular calcium and phosphate concentrations: a potential mechanism for accelerated vascular calcification in ESRD. J Am Soc Nephrol, 2004. 15(11): p. 2857-67.

113. Blacher, J., et al., Arterial calcifications, arterial stiffness, and cardiovascular risk in end-stage renal disease. Hypertension, 2001. 38(4): p. 938-42.

114. London, G.M., et al., Arterial media calcification in end-stage renal disease: impact on all-cause and cardiovascular mortality. Nephrol Dial Transplant, 2003. 18(9): p. 1731-40.

115. Proudfoot, D. and C.M. Shanahan, Biology of calcification in vascular cells: intima versus media. Herz, 2001. 26(4): p. 245-51.

116. Essalihi, R., et al., Regression of medial elastocalcinosis in rat aorta: a new vascular function for carbonic anhydrase. Circulation, 2005. 112(11): p. 1628-35.

117. Bas, A., et al., Reversibility of calcitriol-induced medial artery calcification in rats with intact renal function. J Bone Miner Res, 2006. 21(3): p. 484-90.

118. Price, P.A., A.M. Roublick, and M.K. Williamson, Artery calcification in uremic rats is increased by a low protein diet and prevented by treatment with ibandronate. Kidney Int, 2006. 70(9): p. 1577-83.

119. US Renal Data System. USRDS 1998 annual data report. Bethesda: National Institute of Diabetes and Kidney Diseases 1999: p. 63-90.

120. US Renal Data System. USRDS 1998 annual data report. Bethesda: National Institute of Diabetes and Kidney Diseases 1999: p. 79-90.

121. Raggi, P., et al., Cardiac calcification in adult hemodialysis patients. A link between end-stage renal disease and cardiovascular disease? J Am Coll Cardiol, 2002. 39(4): p. 695-701.

122. Goodman, W.G., et al., Coronary-artery calcification in young adults with end-stage renal disease who are undergoing dialysis. N Engl J Med, 2000. 342(20): p. 1478-83.

123. Pilkey, R.M., et al., Subclinical vitamin K deficiency in hemodialysis patients. Am J Kidney Dis, 2007. 49(3): p. 432-9.

124. Holden, R.M. and S.L. Booth, Vascular calcification and chronic kidney diseases; the role of vitamin K. . Nat Clin Pract Nephrol, 2007. 3(10): p. 522-3. 


\section{CHAPTER 2}

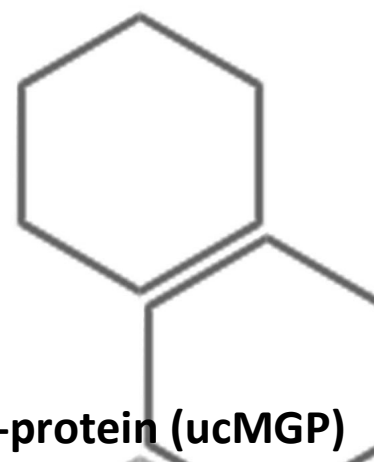

\section{as a biomarker for cardiovascular calcification}

Ellen C.M. Cranenburg ${ }^{1,2}$, Cees Vermeer ${ }^{1,2}$, Ralf Koos ${ }^{3}$, Marie-Louise Boumans ${ }^{1}$, Tilman M. Hackeng $^{1}$, Freek G. Bouwman ${ }^{4}$, Martijn Kwaijtaal ${ }^{2}$, Vincent M. Brandenburg ${ }^{5}$, Markus Ketteler ${ }^{5}$, and Leon J. Schurgers ${ }^{1,2}$

${ }^{1}$ VitaK and ${ }^{2}$ Cardiovascular Research Institute Maastricht (CARIM), Maastricht University, Maastricht, the Netherlands; ${ }^{3}$ Department of Cardiology, RWTH University Hospital Aachen, Aachen, Germany; ${ }^{4}$ Department of Human Biology NUTRIM, Maastricht University, Maastricht, the Netherlands; ${ }^{5}$ Department of Nephrology and Clinical Immunology, RWTH University Hospital Aachen, Aachen, Germany

Based on: J Vasc Res 2008; 45(5): 427-36 


\begin{abstract}
Matrix $\mathrm{p}$-carboxyglutamate (Gla)-protein (MGP) is a vitamin K-dependent protein and a strong inhibitor of vascular calcification. Vitamin $\mathrm{K}$ deficiency leads to synthesis of inactive uncarboxylated MGP species (ucMGP), which accumulate at sites of arterial calcification. We hypothesized that as a result of UcMGP deposition around arterial calcification, the circulating fraction of UcMGP is decreased. Here we report on the development of an UCMGP assay and the potential diagnostic utility of monitoring serum ucMGP levels.
\end{abstract}

An enzyme-linked immunosorbent assay (ELISA) was developed with which circulating total ucMGP ( $t$-ucMGP) can be determined. Serum t-ucMGP levels were measured in healthy subjects $(n=165)$ and in four patient populations; patients who underwent coronary angioplasty $(n=30)$, patients with aortic stenosis $(n=25)$, hemodialysis $(n=52)$, and calciphylaxis patients $(n=10)$. All four patient populations had significantly lower $t$-ucMGP levels. In angioplasty patients and in those with aortic stenosis some overlap was observed with the control population. However, in the hemodialysis and calciphylaxis populations virtually all subjects had t-ucMGP levels below the normal adult range.

Serum t-ucMGP may be used as a biomarker to identify those at risk for developing vascular calcification. This assay may become an important tool in the diagnosis of cardiovascular calcification. 


\section{The circulating inactive form of \\ Matrix Gla-protein (ucMGP) as a biomarker \\ for cardiovascular calcification}

\section{Introduction}

Vitamin K-dependent proteins are present in blood and in a wide variety of tissues throughout the body; they exert a broad range of functions, for example as blood coagulation factors in haemostasis [1]. In recent years one of these proteins has gained increasing attention: matrix $\gamma$-carboxyglutamate (Gla)-protein (MGP), a potent inhibitor of calcification present in cartilage and the vessel wall, where it is synthesized by chondrocytes and vascular smooth muscle cells (VSMC), respectively [2, 3]. MGP contains 9 glutamate residues, 5 of which may be $\gamma$-carboxylated in a posttranslational step catalyzed by the vitamin $\mathrm{K}$ dependent $\mathrm{\gamma}$-glutamate carboxylase [1]. Deficiency of vitamin $\mathrm{K}$, either due to nutritional deficiency or the use of coumarin-derivatives, results in uncarboxylation of MGP and impairment of its biological function [4].

With immunohistochemical techniques based on monoclonal antibodies specifically recognizing carboxylated and uncarboxylated MGP species (cMGP and UcMGP, respectively), we have recently demonstrated the massive accumulation of UCMGP in atherosclerotic lesions and areas of calcification [5]. This has been a consistent finding in several studies. Price et al. reported the accumulation of UcMGP at sites of arterial calcification in rats treated with vitamin D and warfarin [6] and Sweatt et al. showed that the aortic wall of aging rats contained elevated concentrations of ucMGP [7]. It was proposed that ucMGP could bind to apatite present in calcifying tissues irrespective of its Gla-residues, which was supported by the fact that thermal decarboxylation of MGP in vitro did not affect its affinity for hydroxyapatite [8].

The ability of MGP to inhibit calcification was demonstrated in transgenic MGP deficient mice. The MGP null mice were born normally, but all developed severe medial arterial calcifications and subsequent vascular ruptures leading to death within 6 weeks [9]. This vascular phenotype could only be rescued if MGP was restored locally in the VSMC and if MGP was $y$-carboxylated [10], suggesting that MGP is a local inhibitor of vascular calcification and that its function depends on the presence of Gla residues, respectively. Glaresidues are able to bind calcium both in solution and in calcium salt crystals, which is thought to be associated with the ability of MGP to inhibit crystal growth $[8,11]$. Moreover, it has been suggested that its mechanism of action is based on the binding of MGP to BMP-2 $[12,13]$, a bone morphogenetic protein. MGP can also bind to vitronectin, a multifunctional protein present in plasma and extracellular matrix [14]. Nishimoto et al. proposed that this binding may assist cells in developing tissues to migrate and differentiate [14]. 
In humans, loss-of-function mutations in the MGP gene result in the Keutel syndrome (KS)[15]: a recessive disorder characterized by abnormal cartilage calcification, peripheral pulmonary artery stenoses, brachytelephalangism, and neural hearing loss [16]. Postmortem examination of one of the first described KS patients has revealed calcification of arteries throughout the body [17]. The propensity to develop arterial calcification is present in a wide range of other patient populations including patients with diabetes, atherosclerosis, and renal dysfunction. Additionally, patients using oral anticoagulants (coumarins) have significantly increased aortic valve calcification and coronary artery calcification $[18,19]$. Patients with moderate to severe arterial calcification (as assessed by multislice computed tomography [MSCT] scanning) have an unfavorable prognosis compared to patients with no or mild calcification [20-22]. Therefore, the measurement of biomarkers which can predict or reflect the disease becomes of substantial importance.

The first commercially available MGP assay does not discriminate between_carboxylated and uncarboxylated MGP [23], but detects total desphosphorylated (dp) MGP species. Using this t-dpMGP assay, we have reported increased as well as decreased circulating MGP levels in patients suffering from various forms of atherosclerotic disease [5, 23, 24]. A correlation between t-dpMGP levels and coronary artery calcification (CAC) was found in subjects with suspected coronary artery disease [25]; however, no correlation with CAC could be established in patients with chronic kidney disease (CKD) [26]. Additionally, in CKD patients increased levels have been described [24], as well as no significant difference in MGP levels between these patients and control subjects [27]. Thus, although this assay performs well, results are sometimes conflicting and the strong overlap between serum t-dpMGP levels in patients and the normal range does not allow its use for individual diagnosis.

We have developed a competitive enzyme-linked immunosorbent assay (ELISA) which specifically recognizes ucMGP species. The accumulation of MGP in its uncarboxylated form at sites of arterial calcification and its probable high affinity for hydroxyapatite $[5,7$, 8], suggest that ucMGP species are not set free into the circulation from calcified arteries. We hypothesized that in the presence of arterial calcification (arterial medial calcification or in atherosclerotic lesions), the levels of circulating ucMGP species are decreased. To test our hypothesis and to evaluate the potential diagnostic utility of the assay we have measured total ucMGP levels (t-ucMGP) in serum and plasma from different patient cohorts. 


\section{The circulating inactive form of \\ Matrix Gla-protein (ucMGP) as a biomarker \\ for cardiovascular calcification}

\section{Materials and methods}

\section{Subjects}

Apparently healthy subjects, 91 males and 74 females, were recruited from the general Maastricht population for the assessment of the normal range and reference. Patient samples were acquired from a total of 117 subjects: 52 hemodialysis patients and 10 calciphylaxis patients from the department of Nephrology and Clinical Immunology of the University Hospital Aachen, 25 aortic stenosis patients from the department of Cardiology of the University Hospital Aachen, and 30 patients with a history of recent coronary angioplasty from the University Hospital Maastricht. An exclusion criterion for both the reference population and the patient groups was the use of coumarin derivatives. The study was approved by the local Medical Ethics Committee, and informed consent was obtained from all participants.

\section{Serum handling}

Blood was collected by venipuncture in serum tubes $(10 \mathrm{ml}$; BD Vacutainer systems, Plymouth, UK) and in sodium citrate (10 ml; BD Vacutainer systems, Plymouth, UK) and stored for 20 minutes at room temperature before centrifugation (15 min $1580 \mathrm{~g}$ ). Serum and plasma were sub-sampled in $250 \mu \mathrm{l}$ aliquots and frozen at $-80^{\circ} \mathrm{C}$ until testing.

\section{Materials}

All chemicals and reagents described below, were of analytical grade or better and obtained from commercial suppliers. Synthetic peptides homologous to the uncarboxylated MGP sequences 35-49 and 35-54 (designated as ucMGP ${ }^{35-49}$ and ucMGP $^{35-54}$, respectively) and carboxylated MGP sequences 35-49 and 35-54 (CMGP ${ }^{35-49}$ and $\mathrm{CMGP}^{35-54}$ ) were made by Pepscan (Lelystad, The Netherlands). All peptides were purified and analyzed by LC/MS (ionspray, positive ion detection) and their purity level was $>98 \%$ for all peptides. Synthetic full length MGP, cMGP or ucMGP [28] was used for antibody screening and to define assay specificity. The tracer for the assay was prepared by biotinylating the non-carboxylated 3554 peptide ( $u c M G P^{35-54}$ ) using the EZ-Link Maleimide $\mathrm{PEO}_{2}$-Biotin kit according to the manufacturer's instructions (Pierce, Etten-Leur, the Netherlands).

\section{t-ucMGP assay}

The monoclonal antibody against ucMGP used for serum measurements was provided by VitaK BV (Maastricht, The Netherlands); the same antibody has been used previously for immunohistochemical staining. $[5,29]$ The monoclonal antibody (IgG1 isotype, designated as mAb-ucMGP) was raised against the human ucMGP Gla-domain (residues 35-49), and selected for its specificity towards ucMGP using standard techniques. [5] Polyclonal Rabbit-antiMouse IgG (Dako, Heverlee, Belgium) was diluted 2000 fold in carbonate buffer $(0.1 \mathrm{~mol} / \mathrm{L}$ sodium carbonate, $\mathrm{pH}$ 9.6) and used for the coating of the microtiter plate (100 $\mu \mathrm{L} /$ well). 
After incubation for 1.5 hours at $37^{\circ} \mathrm{C}$, remaining protein-binding sites were blocked with 200 $\mu \mathrm{L}$ of blocking buffer ( $2 \%$ HNBSA consisting of HEPES-NaCl buffer [175 mM NaCl, $25 \mathrm{mM}$ HEPES, pH 7.7]) and bovine serum albumin (BSA; Sigma, St. Louis, MO) and incubated for 2 hours at room temperature. Next, the plate was washed 5 times with $300 \mu \mathrm{L}$ of washing buffer (0.05\% Tween-20 in HEPES-NaCl buffer) and incubated overnight at $4{ }^{\circ} \mathrm{C}$ with $100 \mu \mathrm{L} \mathrm{mAb}$ ucMGP $(0.69 \mu \mathrm{L} / \mathrm{ml}$ in $2 \%$ HNBSA; VitaK BV). Subsequently, the plate was washed 5 times with $300 \mu \mathrm{L}$ of HEPES-Tween washing buffer. Five $\mu \mathrm{L}$ of either serum sample or standard were diluted in $70 \mu \mathrm{L}$ of $2 \%$ HNBSA and $20 \mu \mathrm{L}$ of this solution were supplemented with $100 \mu \mathrm{L}$ of tracer (biotinylated ucMGP ${ }^{35-54}, 0.7 \mu \mathrm{g} / \mathrm{mL}$ ). After gentle mixing, $100 \mu \mathrm{L}$ of this solution were transferred to the microtiter plate and incubated overnight at $4^{\circ} \mathrm{C}$. After 5 washing cycles with $300 \mu \mathrm{L}$ of HEPES-Tween washing buffer, the plate was incubated with $100 \mu \mathrm{L}$ Streptavidineperoxide (Zymed, Breda, The Netherlands) diluted 10,000-fold with 0.5\% HNBSA. The plate was subsequently washed 5 times with $300 \mu \mathrm{L} 0.05 \%$ HEPES-Tween washing buffer and stained with $100 \mu \mathrm{L}$ of 3,3',5,5'tetramethylbenzidine (KPL, Gennep, the Netherlands). The staining process was stopped by adding $50 \mu \mathrm{L}$ of $1.0 \mathrm{~mol} / \mathrm{L} \mathrm{H}_{2} \mathrm{SO}_{4}$, and the plate was read at $450 \mathrm{~nm}$. The ucMGP concentration was calculated with the aid of a calibration curve prepared with synthetic full length ucMGP.

\section{Commercially available MGP assay}

Serum t-dpMGP concentrations were quantified with the kit from Biomedica (Vienna, Austria). The kit is based on the competitive ELISA principle, with antibodies against dpMGP coated on the microtiter plate as has been described before. [24]

\section{Immunoprecipitation}

ucMGP species from plasma were purified by immunoprecipitation according to the method described by Michielsen et al. [30]. In brief, $200 \mu \mathrm{g}$ of mAb-ucMGP were incubated for 1 hour at room temperature with $100 \mathrm{mg}$ of protein A-sepharose (Pharmacia Biotech, Uppsala, Sweden). After incubation, antibodies were cross-linked with dimethyl pimelimidate (Sigma) and remaining reactive amino groups were quenched. The beads were added to a normal pool plasma sample $(15 \mathrm{ml})$ and incubated overnight at $4{ }^{\circ} \mathrm{C}$. After washing of the beads with PBS, the bound fraction was collected by adding $0.1 \mathrm{M}$ Glycine, $\mathrm{pH}$ 2.7. The immunoprecipitated proteins were analyzed on SDS-polyacrylamide gel electrophoresis (-PAGE). One SDS-PAGE gel was used to transfer proteins to Immobilon-P membrane for Western blot analysis using either a polyclonal antibody raised against the MGP C-terminus (designated as $\mathrm{pAb}-\mathrm{MGP}^{61-79}$ ) or a monoclonal antibody raised against the MGP N-terminal peptide 3-15 (designated as mAb-MGP ${ }^{3-15}$; VitaK BV). 


\section{The circulating inactive form of \\ Matrix Gla-protein (ucMGP) as a biomarker \\ for cardiovascular calcification}

Mass Spectrometry analysis

A second SDS-PAGE gel was used to collect the protein fraction for analysis by mass spectrometry. The MGP protein band was excised from the SDS-PAGE gel and processed on a MassPREP digestion robot (Waters). Generation of tryptic digest from the protein by ingel digestion, MALDI-TOF analysis and subsequent database searching were performed as described previously. [31] For MS/MS analysis, $1.0 \mu \mathrm{L}$ of the peptide mixture and $1.0 \mu \mathrm{L}$ matrix solution $(2.5 \mathrm{mg} / \mathrm{mL}$ CHCA in $50 \%$ acetonitrile/0.1\% trifluoroacetic acid (TFA)) were spotted on a 384 wells target plate of a MALDI-TOF/TOF (4800 MALDI TOF/TOF analyzer, Applied Biosystems). Typically, 500 shots were combined for obtaining a spectrum. The generated peak list was searched with the MASCOT search engine v2.0 against the SwissProt protein database for protein identification. One miss-cleavage was tolerated; carbamidomethylation was set as a fixed modification and oxidation of methionine as an optional modification. The protein charge was set at $1+$. The peptide mass tolerance was set to $1 \mathrm{D}$ and the MS/MS tolerance was set at $0.2 \mathrm{D}$. No restrictions were made on the protein mass. A protein was regarded identified when it had a significant Mascot probability score $(p<0.05)$.

\section{Statistical analysis}

Since t-ucMGP concentrations were not normally distributed, non-parametric testing was used. Non-parametric correlations (Spearman's correlation) were used to test for differences between plasma and serum ucMGP levels, and to determine the correlation with age. Healthy subjects were divided into 3 groups: subjects aged $<40$ years, between 40 and 60 years, and $\geq 60$ years. The Kruskal-Wallis test was used to test for differences between the 3 groups of healthy subjects. Mann-Whitney $U$ tests were used to test for differences between the reference population and the different patient populations. Unless stated otherwise, all data are means of duplicate measurements and presented as means \pm SD. Data processing and statistical analysis was performed using SPSS for Windows (SPSS Corp, Chicago, IL, USA). Significance levels were based on two-tailed tests, with alpha level set at 0.05 . 


\section{Results}

\section{Characteristics of the ucMGP assay}

The intra-assay variability was tested for two different serum samples (t-ucMGP levels: 697 and $303 \mathrm{nmol} / \mathrm{L}$ ) with 21 replicates per sample in the same plate. The variation coefficients were found to be $7.2 \%$ and $10.6 \%$, respectively. The inter-assay variability was determined using pooled serum from 9 healthy subjects which was measured in duplicate on 21 different plates from different lot numbers on subsequent working days. The interassay variation coefficient was found to be $11.4 \%$.

The lower detection limit of the assay was calculated as $3 \times \mathrm{SD}$ of the zero standard obtained from 21 replicates in one plate, and was found to be $21 \mathrm{nmol} / \mathrm{L}$. We compared the difference between citrated plasma and serum ucMGP measurements in paired samples from healthy subjects and patients, and found a good correlation $(r=0.897 ; p<0.001)$.

Figure 1: Recovery after dilution

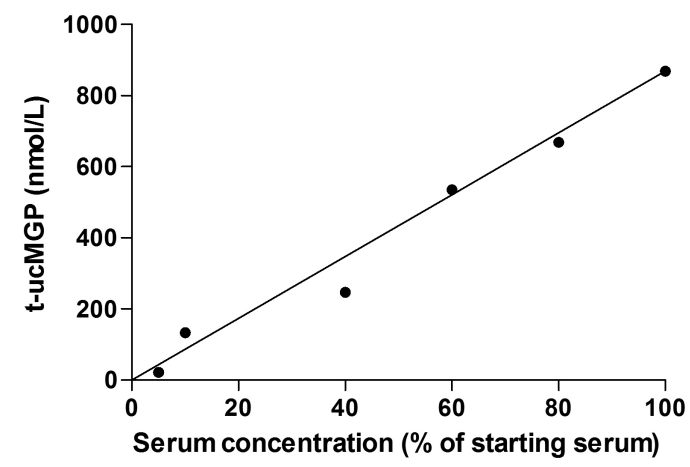

Recovery of t-ucMGP after dilution of serum. Serum from a healthy subject (t-ucMGP $869 \mathrm{nmol} / \mathrm{L}$ ) was diluted with $2 \%$ HNBSA. The theoretical $(-)$ as well as the observed ucMGP concentration $(\bullet)$ is shown. The points represent means of duplicate measurements. 


\section{The circulating inactive form of \\ Matrix Gla-protein (ucMGP) as a biomarker \\ for cardiovascular calcification}

The recovery after dilution was assessed by serially diluting serum from a healthy subject (mean t-ucMGP $869 \mathrm{nmol} / \mathrm{L}$ ) with dilution buffer (2\% HNBSA) as depicted in Figure 1. Further, we assessed spiking recovery and spiked serum samples depleted from MGP by immunoprecipitation (t-ucMGP $1.7 \mathrm{nmol} / \mathrm{L}$ ) with either synthetic full length ucMGP or CMGP. The ratio between the expected and observed t-ucMGP values was $64 \%$ for full length uCMGP and $<2.5 \%$ for full length CMGP, as measured with the t-ucMGP assay.

Figure 2: Reactivity of mAb-ucMGP with synthetic MGP-derived peptides

A

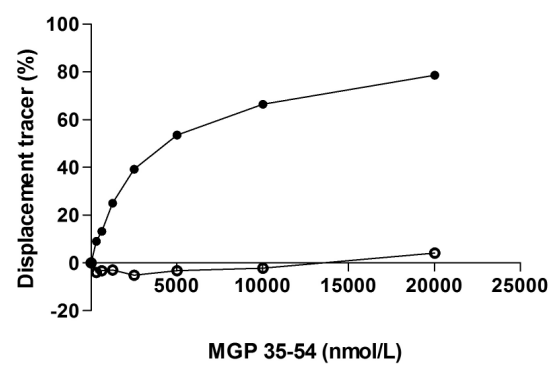

B

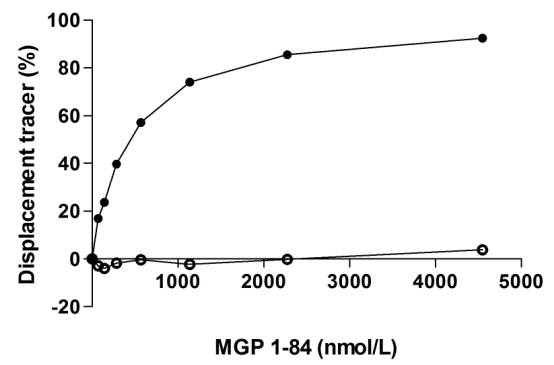

Reactivity of the mAb-ucMGP with synthetic MGP-derived peptides (A) and synthetic full length MGP (B). Serum was mixed with either uncarboxylated $(\bullet)$ or carboxylated species $(\circ)$ before testing. Points represent mean $\pm S D(n=12)$.

To assess the assay specificity, mAb-ucMGP was tested for its cross reaction with synthetic MGP peptides (CMGP ${ }^{35-54}$ and ucMGP ${ }^{35-54}$; Figure $2 \mathrm{~A}$ ) and synthetic full length MGP (either cMGP or ucMGP; Figure 2B). These synthetic polypeptides were used in competitive inhibition experiments with the biotinylated ucMGP tracer (ucMGP ${ }^{35-54}$ ). As shown in Figure 2, both ucMGP ${ }^{35-54}$ and full length ucMGP were competitive inhibitors in the assay, whereas $\mathrm{CMGP}^{35-54}$ and full length $\mathrm{CMGP}$ were not. Moreover, we tested the synthetic peptides $\mathrm{CMGP}^{35-49}$ and $\mathrm{uCMGP} \mathrm{P}^{35-49}$, in which 3 glutamate (Glu) residues are present. Both peptides did not bind to the mAb-ucMGP (data not shown). Therefore, the mAb-ucMGP recognizes only ucMGP with at least 4 Glu residues. It was concluded that only uCMGP is recognized by the mAb-ucMGP used in our conformation-specific assay. 
Identification of the product immunoprecipitated with the mAb-ucMGP was performed on Western blot analysis and showed a single band at $11 \mathrm{kD}$, which reacted positively with a monoclonal antibody against the $\mathrm{N}$-terminus and with a polyclonal antibody against the C-terminus of MGP (Figure 3). Analysis of the 11 kD-band of the second SDSPAGE gel on MALDI-TOF/TOF after in-gel digestion revealed ucMGP as protein identity in the immunoprecipitated product (Table 1).

Figure 3: Western blot analysis

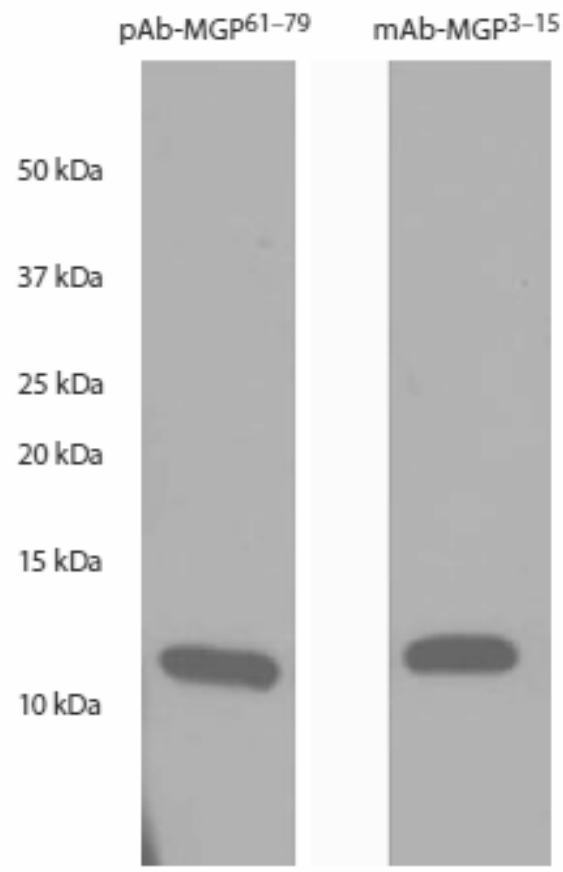

Western blot analysis of the immunoprecipitated product shows a single band at $\sim 11 \mathrm{kD}$ as detected with both a polyclonal antibody raised against the MGP C-terminus (pAb-MGP ${ }^{61-79}$ ) and a monoclonal antibody raised against the MGP N-terminus (mAb-MGP ${ }^{3-15}$ ). 
The circulating inactive form of

Matrix Gla-protein (ucMGP) as a biomarker

for cardiovascular calcification

Table 1: Identification of ucMGP by MALDI TOF/TOF

\begin{tabular}{|c|c|c|c|c|c|c|}
\hline Protein ID & & MS & & MS/MS & & \\
\hline $\begin{array}{l}\text { Accession } \\
\text { number }\end{array}$ & Protein name & MASCOT score & $\begin{array}{l}\text { Sequence } \\
\text { coverage }\end{array}$ & $\begin{array}{l}\text { Matched } \\
\text { peptides }\end{array}$ & $\begin{array}{l}\text { Peptide } \\
\text { sequence }\end{array}$ & $\begin{array}{l}\text { MASCOT } \\
\text { score }\end{array}$ \\
\hline P08493 & Matrix Gla-protein & 62 & $32 \%$ & 3 & $\begin{array}{l}\text { SKPVHELNR } \\
\text { NANTFISPQQR }\end{array}$ & 47 \\
\hline
\end{tabular}

After excision of the 11 kD-protein band from the second SDS-PAGE gel followed by in-gel digestion, the obtained mixture of peptides was analyzed by mass-spectrometry (MS); the resulting collection of masses (peptide fingerprint) was searched with the MASCOT search engine against the Swiss-Prot protein database. Matrix Glaprotein (MGP) was identified with a significant MASCOT probability score of 62 and sequence coverage of $32 \%$. To verify the identification of MGP, tandem mass spectrometry sequencing (MS/MS analysis) was performed; two peptide sequences of the three matched peptides could be determined with a significant MASCOT probability score of 47 , confirming that UCMGP is indeed the protein identity in the immunoprecipitated product.

\section{Sample preparation}

To further characterize our assay, the influence of variations in the sample preparation procedure was tested using pooled serum from 20 healthy subjects (mean t-ucMGP $543 \pm 23$ $\mathrm{nmol} / \mathrm{L}$ ). There was no measurable effect of centrifugation speed (1580 and $8000 \mathrm{~g}$ ) during serum preparation (mean t-ucMGP $560 \pm 10 \mathrm{nmol} / \mathrm{L}$ and $526 \pm 22 \mathrm{nmol} / \mathrm{L}$, respectively). Freeze-thawing up to 10 cycles did not affect the observed ucMGP concentration (mean tucMGP after 10 cycles $554 \pm 38 \mathrm{nmol} / \mathrm{L})$.

Normal range, within-day variations, and day-to-day variations

The healthy subjects were divided into 3 groups: subjects under the age of 40 (mean age $30 \pm 6$ years), those aged 40 to 60 years (mean age $51 \pm 6$ years) and those aged 60 years or more (mean age $68 \pm 11$ years). Baseline characteristics of the healthy subjects are shown in Table 2. The normal range for t-ucMGP was established in the group of 54 healthy males and females under the age of 40 (reference population), with a mean serum $t$ ucMGP level of $504 \pm 98 \mathrm{nmol} / \mathrm{L}$. The normal range of t-ucMGP levels, defined as the mean $\pm 2 \times S D$, was calculated to be between 308 and $699 \mathrm{nmol} / \mathrm{L}$; no difference was observed between males and females. The mean level of serum t-ucMGP in the group of subjects aged 40 to 60 years was $481 \pm 77 \mathrm{nmol} / \mathrm{L}$; in the group of subjects aged 60 years or more it was $441 \pm 97 \mathrm{nmol} / \mathrm{L}$. The mean values of these two groups were within the normal range, with tailing towards lower values (Figure 4). There was a significant difference in tucMGP levels between the three groups of healthy subjects $(p=0.02)$; additionally, an inverse correlation with age in the total population of healthy subjects was found $(r=-$ $0.81 ; p=0.02$ ). 


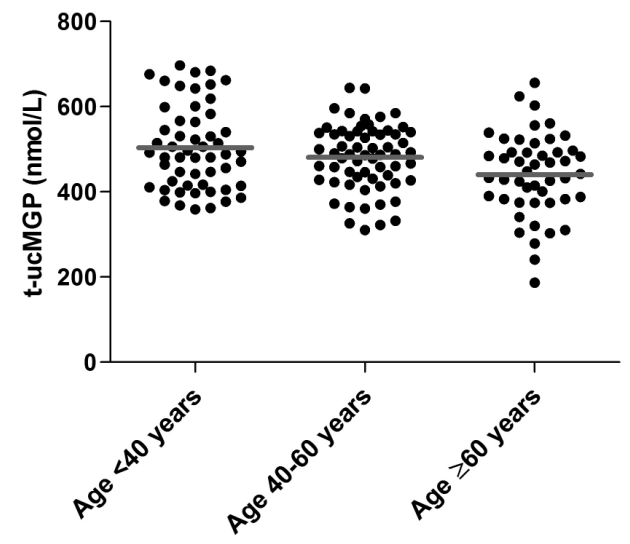

Serum t-ucMGP levels in the reference subjects, divided in 3 groups: subjects aged less than 40 years, aged 40 to 60 years and aged 60 or more. Mean \pm SD ucMGP levels were $504 \pm 98,481 \pm 77$, and $441 \pm 97 \mathrm{nmol} / \mathrm{L}$ respectively (depicted as horizontal bars). The difference between the 3 subpopulations was significant at $p=0.02$. The normal range, defined as the mean $\pm 2 \times S D$, was established in the group of subjects aged less than 40 years (reference population) and was calculated to be between 308 and $699 \mathrm{nmol} / \mathrm{L}$.

Table 2: Demographic and medical characteristics of healthy subjects

\begin{tabular}{lll}
\hline Reference population & Total & Aged < 40 years \\
\hline $\mathrm{N}$ & 165 & 54 \\
\hline Demographic characteristics & & \\
\hline Age (years) & $49 \pm 16$ & $30 \pm 6$ \\
BMI (kg/m²) & $23 \pm 4$ & $22 \pm 3$ \\
Gender (m/f) & $91 / 74$ & $29 / 25$ \\
\hline Clinical characteristics & & \\
\hline $\begin{array}{l}\text { Diabetes (number [\%]) } \\
\text { Hypertension (number [\%]) }\end{array}$ & $0(0)$ & $0(0)$ \\
History of heart disease (number [\%]) & $0(0)$ & $0(0)$ \\
\hline Medication use (number [\%]) & $0(0)$ & $0(0)$ \\
\hline
\end{tabular}

Data are given either as absolute values \pm SD or as numbers, with percentage of total between parentheses. Abbreviation used: BMI, body mass index. 


\section{The circulating inactive form of \\ Matrix Gla-protein (ucMGP) as a biomarker \\ for cardiovascular calcification}

The time-related variability of serum t-ucMGP was established in 4 healthy subjects (between 25 and 35 years old, 2 males and 2 females; mean t-ucMGP $578 \pm 233 \mathrm{nmol} / \mathrm{L}$ ) from whom blood was taken by venipuncture at 9 time points on one day and subsequently on 5 different days at 9 AM with 1-week intervals. The within-day variation was calculated for each subject separately by expressing the SD as a percentage of the mean of the 9 time points. Coefficients of variation for each subject were found to be $6.6 \%$ (mean t-ucMGP

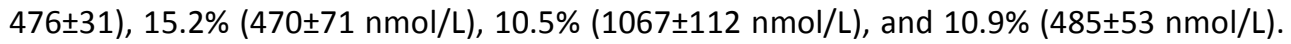
There was no observation of a distinct circadian pattern. In a similar way, the day-to-day variation was calculated from the 6 samples obtained at weekly intervals and coefficients of variation were found to be $14.7 \%(513 \pm 75 \mathrm{nmol} / \mathrm{L}), 9.2 \%(449 \pm 41 \mathrm{nmol} / \mathrm{L}), 10.5 \%$ $(1016 \pm 107 \mathrm{nmol} / \mathrm{L})$ and $10.7 \%(511 \pm 55 \mathrm{nmol} / \mathrm{L})$.

\section{Patient samples}

To evaluate the potential clinical utility of the t-ucMGP assay, we measured serum tucMGP in 4 different patient populations. The demographic and clinical characteristics of the patient populations are summarized in Table 3. Figure 5 shows the serum t-ucMGP distribution in the reference (mean t-ucMGP $504 \pm 98 \mathrm{nmol} / \mathrm{L}$ ) and coronary angioplasty $(389 \pm 182 \mathrm{nmol} / \mathrm{L})$, aortic stenosis $(312 \pm 109 \mathrm{nmol} / \mathrm{L})$, dialysis $(172 \pm 82 \mathrm{nmol} /)$, and calciphylaxis $(140 \pm 55 \mathrm{nmol} / \mathrm{L}$ ) population. Obviously, all 4 patient populations had significantly lower circulating t-ucMGP levels in comparison with the reference population (healthy subjects under the age of 40 ). Both the angioplasty population and aortic stenosis population showed a broader range as compared to the reference population with a strong tailing towards lower values. The t-ucMGP levels in hemodialysis as well as in calciphylaxis patients showed virtually no overlap with the normal range (Figure 5). 
Figure 5: t-ucMGP levels in the patient populations

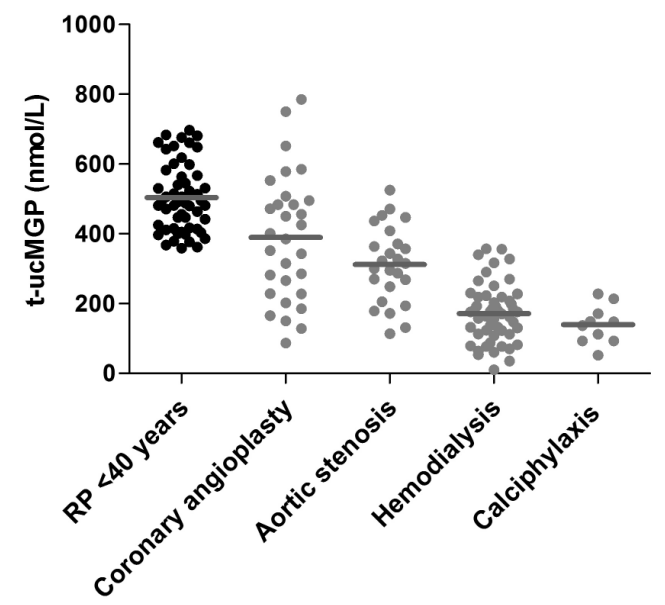

Serum t-ucMGP levels in patients compared to the reference population (healthy subjects aged less than 40 years). Mean $\pm S D$ t-ucMGP values of angioplasty, aortic stenosis, dialysis and calciphylaxis patients were $389 \pm 182,312 \pm 109,172 \pm 82$, and $140 \pm 55 \mathrm{nmol} / \mathrm{L}$, respectively (depicted as horizontal bars). Differences between the reference population and angioplasty, aortic stenosis, dialysis and calciphylaxis patients were statistically significant at $p=0.002, p<0.001, p<0.001$, and $p<0.001$, respectively. 


\section{The circulating inactive form of \\ Matrix Gla-protein (ucMGP) as a biomarker \\ for cardiovascular calcification}

Table 3: Demographic and medical characteristics of patients

\begin{tabular}{lllll}
\hline Patient populations & Coronary angioplasty & Aortic stenosis & Hemodialysis & Calciphylaxis \\
\hline $\mathrm{N}$ & 30 & 25 & 52 & 10 \\
\hline Demographic characteristics & & & & \\
\hline Age (years) & $52 \pm 8$ & $72 \pm 10$ & $64 \pm 13$ & $63 \pm 14$ \\
BMI (kg/m²) & $27 \pm 4$ & $28 \pm 4$ & $27 \pm 4$ & - \\
Gender (m/f) & $18 / 12$ & $14 / 11$ & $28 / 24$ & $5 / 5$ \\
\hline Clinical characteristics & & & & $4(40)$ \\
\hline $\begin{array}{l}\text { Diabetes (number [\%]) } \\
\text { Hypertension (number [\%]) }\end{array}$ & $5(15)$ & $7(28)$ & $18(35)$ & $9(90)$ \\
CAD (number [\%]) & $6(20)$ & $16(64)$ & $46(88)$ & $8(80)$ \\
CKD (number [\%]) & $30(100)$ & $15(60)$ & $28(54)$ & $10(100)^{2}$ \\
\hline
\end{tabular}

Data are given either as absolute values \pm SD or as numbers, with percentage of total between parentheses. Abbreviations used: BMI, body mass index; CAD, coronary artery disease; CKD, chronic kidney disease.

${ }^{1}$ All 52 hemodialysis patients were by definition in CKD stage $5 ;{ }^{2}$ seven patients in the calciphylaxis group were dialysis patients (CKD stage 5: 6 hemodialysis, 1 peritoneal dialysis), three patients were renal transplant recipients (CKD stage $3-4)$.

Additionally, t-ucMGP levels of the patient populations were compared with the group of healthy subjects of the same age. The t-ucMGP level of the angioplasty population was significantly lower in comparison with the group of healthy subjects aged 40 to 60 years $(p=0.003)$. The t-ucMGP levels of the aortic stenosis, dialysis, and calciphylaxis populations were significantly lower as compared to the group of healthy subjects aged 60 years or more $(p<0.001, p<0.001$, and $p<0.001$, respectively). These results indicate that low ucMGP levels in the patient populations are not merely an effect of age.

Finally, t-dpMGP levels were measured with the commercially available MGP assay in the 4 patient populations. Only the group means of aortic stenosis and calciphylaxis patients were significantly different from the reference population (data not shown). Additionally, more than $70 \%$ of the $t$-dpMGP levels in the patients showed overlap with the normal range. These data do not allow individual risk assessment, since the majority of the patients cannot be discriminated from the healthy reference population. 


\section{Discussion}

In this paper, we report on the development of an ELISA-based competitive assay for human total circulating ucMGP (t-ucMGP) species. To test the assay for its diagnostic utility, we analysed ucMGP in 4 different patient populations. Circulating t-ucMGP levels were significantly lower in both groups of cardiovascular patients as well as in dialysis and calciphylaxis patients. Moreover, t-ucMGP levels in the dialysis and calciphylaxis patients did not overlap with those in the reference population. Clearly, serum t-ucMGP is a marker capable of discriminating between healthy subjects and patients with end-stage renal disease.

Dialysis patients have a 60 to $80 \%$ prevalence of moderate to severe vascular calcifications [32-34]. Calciphylaxis is by definition characterized by extensive calcifications of cutaneous arterioles usually accompanied by large vessel calcifications [35, 36]. The angioplasty and aortic stenosis patient populations each represent a different vascular pathologic etiology. Aortic stenosis patients have a high incidence of calcification but can be free of atherosclerosis, whereas patients who underwent angioplasty suffer from intimal (atherosclerotic) vascular disease. Additionally, large inter-individual variation in the extent of vascular calcification can be expected in these patient groups and could explain the overlap with the reference population.

The specificity of the assay for ucMGP species was demonstrated by using synthetic MGP peptides; only the ucMGP peptides reacted with the MAb-ucMGP, whereas no affinity was observed for CMGP peptides. Recovery experiments using the same peptides to spike serum samples showed that only the recovery of UCMGP, and not of CMGP, was adequate. Full length MGP was identified by mass spectrometry as the major MGP species recognized by the mAb-ucMGP.

We hypothesized that in the presence of arterial calcification (arterial medial calcification or in atherosclerotic lesions), the circulating fraction of ucMGP may be decreased. The results obtained with our t-uCMGP assay show that in patient populations known for cardiovascular calcification serum t-ucMGP levels are significantly lower as compared to an apparently healthy reference population. The accumulation of ucMGP species in atherosclerotic lesions and areas of calcification has been reported in several studies [6, 7], including our recent study using immunohistochemical techniques [5]. The balance between tissue ucMGP and circulating ucMGP species in calcified arteries may be different from that in healthy ones with increased t-ucMGP levels in calcified tissues and consequently less ucMGP secretion in the circulation. 


\section{The circulating inactive form of \\ Matrix Gla-protein (ucMGP) as a biomarker \\ for cardiovascular calcification}

However, the exact mechanisms - including the process of MGP secretion in the circulation - remain to be elucidated. Alternative explanations for the low ucMGP levels in the selected patient populations may be lower synthesis of MGP and increased degradation of MGP before carboxylation in these patients.

A limitation of our study was the inability to measure circulating levels of cMGP. At the moment there is no assay available to measure this conformation of MGP; in the near future we aim to develop a dual-antibody ELISA for CMGP as well as for ucMGP. Also, the reference population should be demonstrated to be free from disease, e.g. free from cardiovascular calcification; this would require MSCT or electron-beam computed tomography (EBCT) screening of all healthy volunteers. Since these imaging techniques are expensive, and also because they expose subjects to significant X-ray radiation, we have not characterized our reference population by MSCT or EBCT. However, it has been reported that the majority of subjects under the age of 40 have very low coronary artery calcium scores as measured with EBCT, with a mean Agatston score for males of 10 and for females of 9 [37]. Therefore we defined the normal range of circulating t-ucMGP in the group of healthy subjects under the age of 40 .

Increasingly more patient populations at risk for vascular calcification are currently being identified, including young females with systemic lupus erythematosus [38], polycystic ovary syndrome [39], and patients with alkaptonuria [40]. The inactive ucMGP species could have potential to serve as a non-invasive biochemical marker for cardiovascular calcification and possibly contribute to the identification of patients requiring radiological assessment of cardiovascular calcification. Obviously, diagnostic applications of t-ucMGP measurements would require much further study. Seen from a therapeutic perspective, vitamin $\mathrm{K}$ therapy has the potential of increasing the activity of MGP, and possibly reducing the development of calcification. In an animal model system the efficacy of such therapy has recently been demonstrated $[29,41]$. The effect of vitamin $K$ therapy on circulating MGP levels remains to be determined.

The first commercially available MGP assay, measuring t-dpMGP levels, appears to be less suited for individual risk assessment. The cardiovascular patients as well as the patients prone for cardiovascular calcification could not be adequately separated from the reference population, since $73 \%$ of the t-dpMGP levels in these patients were within normal range. $\mathrm{A}$ principal difference with our newly developed t-ucMGP assay is that the commercial assay does not discriminate between carboxylated and uncarboxylated MGP species. 
In conclusion, we have developed a robust assay for circulating t-ucMGP allowing the assessment of the circulating levels of uncarboxylated MGP species. It was found that different patient groups with cardiovascular disease or with a propensity to develop vascular calcification had significantly lower levels of circulating t-ucMGP and could be discriminated from the healthy reference population. Further studies are necessary to determine the exact correlation between t-ucMGP levels and the extent of vascular calcification as well as the predictive value of t-ucMGP measurements. 


\section{The circulating inactive form of \\ Matrix Gla-protein (ucMGP) as a biomarker \\ for cardiovascular calcification}

\section{Acknowledgements}

The authors thank Prof. Dr. J. Rosing for critical reading of the manuscript.

\section{References}

1. Vermeer, C., Gamma-carboxyglutamate-containing proteins and the vitamin K-dependent carboxylase. Biochem J, 1990. 266(3): p. 625-36.

2. Luo, G., et al., The matrix Gla protein gene is a marker of the chondrogenesis cell lineage during mouse development. J Bone Miner Res, 1995. 10(2): p. 325-34.

3. Wallin, R., D. Cain, and D.C. Sane, Matrix Gla protein synthesis and gamma-carboxylation in the aortic vessel wall and proliferating vascular smooth muscle cells--a cell system which resembles the system in bone cells. Thromb Haemost, 1999. 82(6): p. 1764-7.

4. Berkner, K.L. and K.W. Runge, The physiology of vitamin $K$ nutriture and vitamin K-dependent protein function in atherosclerosis. J Thromb Haemost, 2004. 2(12): p. 2118-32.

5. Schurgers, L.J., et al., Novel conformation-specific antibodies against matrix gamma-carboxyglutamic acid (Gla) protein: undercarboxylated matrix Gla protein as marker for vascular calcification. Arterioscler Thromb Vasc Biol, 2005. 25(8): p. 1629-33.

6. Price, P.A., S.A. Faus, and M.K. Williamson, Warfarin-induced artery calcification is accelerated by growth and vitamin D. Arterioscler Thromb Vasc Biol, 2000. 20(2): p. 317-27.

7. Sweatt, A., et al., Matrix Gla protein (MGP) and bone morphogenetic protein-2 in aortic calcified lesions of aging rats. J Thromb Haemost, 2003. 1(1): p. 178-85.

8. Price, P.A., S.A. Faus, and M.K. Williamson, Warfarin causes rapid calcification of the elastic lamellae in rat arteries and heart valves. Arterioscler Thromb Vasc Biol, 1998. 18(9): p. 1400-7.

9. Luo, G., et al., Spontaneous calcification of arteries and cartilage in mice lacking matrix GLA protein. Nature, 1997. 386(6620): p. 78-81.

10. Murshed, M., et al., Extracellular matrix mineralization is regulated locally; different roles of two glacontaining proteins. J Cell Biol, 2004. 165(5): p. 625-30.

11. Price, P.A., et al., The elastic lamellae of devitalized arteries calcify when incubated in serum: evidence for a serum calcification factor. Arterioscler Thromb Vasc Biol, 2006. 26(5): p. 1079-85.

12. Zebboudj, A.F., M. Imura, and K. Bostrom, Matrix GLA protein, a regulatory protein for bone morphogenetic protein-2. J Biol Chem, 2002. 277(6): p. 4388-94.

13. Bostrom, K., et al., Matrix GLA protein modulates differentiation induced by bone morphogenetic protein-2 in C3H1OT1/2 cells. J Biol Chem, 2001. 276(17): p. 14044-52.

14. Nishimoto, S.K. and M. Nishimoto, Matrix Gla protein C-terminal region binds to vitronectin. Co-localization suggests binding occurs during tissue development. Matrix Biol, 2005. 24(5): p. 353-61.

15. Munroe, P.B., et al., Mutations in the gene encoding the human matrix Gla protein cause Keutel syndrome. Nat Genet, 1999. 21(1): p. 142-4.

16. Keutel, J., G. Jorgensen, and P. Gabriel, A new autosomal-recessive hereditary syndrome. Multiple peripheral pulmonary stenosis, brachytelephalangia, inner-ear deafness, ossification or calcification of cartilages. Dtsch Med Wochenschr, 1971. 96(43): p. 1676-81 passim.

17. Meier, M., et al., Tracheobronchial stenosis in Keutel syndrome. Eur Respir J, 2001. 17(3): p. 566-9.

18. Schurgers, L.J., et al., Oral anticoagulant treatment: friend or foe in cardiovascular disease? Blood, 2004. 104(10): p. 3231-2.

19. Koos, R., et al., Relation of oral anticoagulation to cardiac valvular and coronary calcium assessed by multislice spiral computed tomography. Am J Cardiol, 2005. 96(6): p. 747-9. 
20. Rosenhek, R., et al., Predictors of outcome in severe, asymptomatic aortic stenosis. N Engl J Med, 2000. 343(9): p. 611-7.

21. Raggi, P., et al., Progression of coronary calcium on serial electron beam tomographic scanning is greater in patients with future myocardial infarction. Am J Cardiol, 2003. 92(7): p. 827-9.

22. Raggi, P., et al., Prognostic value of coronary artery calcium screening in subjects with and without diabetes. J Am Coll Cardiol, 2004. 43(9): p. 1663-9.

23. Braam, L.A., et al., Assay for human matrix gla protein in serum: potential applications in the cardiovascular field. Arterioscler Thromb Vasc Biol, 2000. 20(5): p. 1257-61.

24. Schurgers, L.J., et al., Characteristics and performance of an immunosorbent assay for human matrix Glaprotein. Clin Chim Acta, 2005. 351(1-2): p. 131-8.

25. Jono, S., et al., Matrix Gla protein is associated with coronary artery calcification as assessed by electronbeam computed tomography. Thromb Haemost, 2004. 91(4): p. 790-4.

26. Moe, S.M., et al., Role of calcification inhibitors in the pathogenesis of vascular calcification in chronic kidney disease (CKD). Kidney Int, 2005. 67(6): p. 2295-304.

27. Mazzaferro, S., et al., Serum levels of calcification inhibition proteins and coronary artery calcium score: comparison between transplantation and dialysis. Am J Nephrol, 2007. 27(1): p. 75-83.

28. Hackeng, T.M., et al., Total chemical synthesis of human matrix Gla protein. Protein Sci, 2001. 10(4): p. 86470.

29. Schurgers, L.J., et al., Regression of warfarin-induced medial elastocalcinosis by high intake of vitamin $K$ in rats. Blood, 2007. 109(7): p. 2823-31.

30. Michielsen, E.C., et al., Highly sensitive immunoprecipitation method for extracting and concentrating lowabundance proteins from human serum. Clin Chem, 2005. 51(1): p. 222-4.

31. Bouwman, F., et al., Evaluation and improving the success rate of protein identification by peptide mass fingerprinting using matrix-assisted laser desorption/ionization time-of-flight mass spectrometry. Rapid Commun Mass Spectrom, 2005. 19(17): p. 2465-8.

32. Raggi, P., et al., Cardiac calcification in adult hemodialysis patients. A link between end-stage renal disease and cardiovascular disease? J Am Coll Cardiol, 2002. 39(4): p. 695-701.

33. Goodman, W.G., et al., Coronary-artery calcification in young adults with end-stage renal disease who are undergoing dialysis. N Engl J Med, 2000. 342(20): p. 1478-83.

34. Block, G.A., et al., Effects of sevelamer and calcium on coronary artery calcification in patients new to hemodialysis. Kidney Int, 2005. 68(4): p. 1815-24.

35. Coates, T., et al., Cutaneous necrosis from calcific uremic arteriolopathy. Am J Kidney Dis, 1998. 32(3): p. 384-91.

36. Mazhar, A.R., et al., Risk factors and mortality associated with calciphylaxis in end-stage renal disease. Kidney Int, 2001. 60(1): p. 324-32.

37. Nasir, K., et al., Coronary artery calcium volume scores on electron beam tomography in 12,936 asymptomatic adults. Am J Cardiol, 2004. 93(9): p. 1146-9.

38. Asanuma, Y., et al., Premature coronary-artery atherosclerosis in systemic lupus erythematosus. N Engl J Med, 2003. 349(25): p. 2407-15.

39. Talbott, E.O., et al., Evidence for an association between metabolic cardiovascular syndrome and coronary and aortic calcification among women with polycystic ovary syndrome. J Clin Endocrinol Metab, 2004. 89(11): p. 5454-61.

40. Phornphutkul, C., et al., Natural history of alkaptonuria. N Engl J Med, 2002. 347(26): p. 2111-21.

41. Spronk, H.M., et al., Tissue-specific utilization of menaquinone-4 results in the prevention of arterial calcification in warfarin-treated rats. J Vasc Res, 2003. 40(6): p. 531-7. 


\section{CHAPTER $\mathbf{3}$}

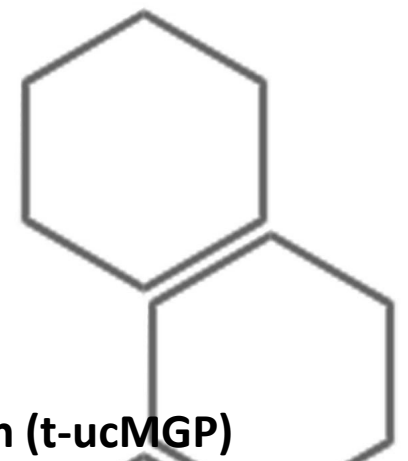

is associated with coronary artery calcification in hemodialysis patients

Ellen C.M. Cranenburg ${ }^{1}$, Vincent M. Brandenburg ${ }^{2}$, Cees Vermeer ${ }^{1}$, Melanie Stenger ${ }^{2}$, Georg Mühlenbruch $^{3}$, Andreas H. Mahnken ${ }^{3}$, Ulrich Gladziwa ${ }^{4}$, Markus Ketteler ${ }^{2}$, and Leon J. Schurgers ${ }^{1}$

${ }^{1}$ VitaK and Cardiovascular Research Institute Maastricht (CARIM), Maastricht University, Maastricht, The Netherlands; ${ }^{2}$ Department of Nephrology and Clinical Immunology, RWTH University Hospital Aachen, Aachen, Germany; ${ }^{3}$ Department of Diagnostic Radiology, RWTH University Hospital Aachen, Aachen, Germany; ${ }^{4}$ Kuratorium für Heimdialyse, Dialysis Center, Würselen, Germany

* The first two authors have contributed equally to this work.

Based on: Thromb Haemost 2009; 101(2): 359-66 


\begin{abstract}
Matrix $\gamma$-carboxyglutamate (Gla)-protein (MGP) is a potent local inhibitor of cardiovascular calcification and accumulates at areas of calcification in its uncarboxylated form (ucMGP). We previously found significantly lower circulating total ucMGP (t-ucMGP) levels in patients with a high vascular calcification burden.
\end{abstract}

Here we report on the potential of circulating t-ucMGP to serve as a biomarker for vascular calcification in hemodialysis (HD) patients. Circulating t-ucMGP levels were measured with an enzyme-linked immunosorbent assay (ELISA) in $40 \mathrm{HD}$ patients who underwent multislice computed tomography (MSCT) scanning to quantify the extent of coronary artery calcification (CAC). The mean t-ucMGP level in HD patients (193 $\pm 65 \mathrm{nmol} / \mathrm{L})$ was significantly lower as compared to apparently healthy subjects of the same age $(441 \pm 97$ $\mathrm{nmol} / \mathrm{L} ; \mathrm{p}<0.001)$ and patients with rheumatoid arthritis (RA) without CAC (560 \pm 140 $\mathrm{nmol} / \mathrm{L} ; \mathrm{p}<0.001)$. Additionally, t-ucMGP levels correlated inversely with CAC scores ( $\mathrm{r}=-$ $0.414, p=0.009$ ), and this correlation persisted after adjustment for age, dialysis vintage, and high-sensitivity C-reactive protein (hs-CRP).

Since circulating t-ucMGP levels are significantly and inversely correlated with the extent of CAC in HD patients, t-ucMGP may become a tool for identifying HD patients with a high probability of cardiovascular calcification. 


\section{Total uncarboxylated Matrix Gla-protein (t-ucMGP) \\ is associated with coronary artery calcification in \\ hemodialysis patients}

\section{Introduction}

Cardiovascular disease (CVD) in patients on hemodialysis (HD) is a clinically outstanding problem: CVD accounts for approximately 50\% of deaths among the dialysis population and cardiovascular mortality is about 10-20 times higher than in the general population $[1,2]$. Arterial intimal calcification is associated with atherosclerotic lesions. Arterial medial calcification is found in diabetic and dialysis patients as well as in most elderly subjects $[3,4]$. Arterial medial calcification was initially thought to be clinically irrelevant, but is now considered to be associated with all-cause and cardiovascular morbidity in dialysis patients, independent of traditional atherogenic risk factors $[5,6]$. Clinically, arterial calcification causes stiffening of the vascular wall, which in turn may result in decreased arterial compliance, increased afterload with development of left ventricular hypertrophy, and decreased coronary perfusion with increased risk for death [5-7].

The exact pathophysiology of arterial calcification in chronic kidney disease (CKD) is not fully understood. Numerous factors have been correlated with arterial calcification in CKD including age, dialysis vintage, and calcium $x$ phosphorus product [8-10]. Since concentrations of calcium and phosphate in extracellular fluid exceed their solubility product under physiological conditions, powerful calcification inhibitors are required to prevent spontaneous calcification of soft tissues throughout the body.

Matrix $\gamma$-carboxyglutamate (Gla)-protein (MGP) is the most potent inhibitor of soft tissue calcification presently known. Its main sources are cartilage and the vessel wall where it is synthesized by chondrocytes and vascular smooth muscle cells (VSMC), respectively [1113]. MGP contains nine glutamate (Glu) residues, five of which can be carboxylated in a vitaminK-dependent reaction to form calcium-binding Gla residues [14]. Additionally, mature MGP contains three serine residues which may be phosphorylated. The function of these phosphoserine residues is presently unknown, but it seems highly probable that they significantly contribute to the calcium-binding properties of MGP [15].

A poor vitamin $\mathrm{K}$ status caused by poor nutritional intake or the use of vitamin Kantagonists (oral anticoagulants), results in the synthesis of uncarboxylated MGP (ucMGP) [14, 16]. After the development of conformation-specific assays for MGP and osteocalcin, a vitamin K-dependent protein present in bone, it has been found that in healthy subjects uncarboxylated species of these proteins are present in the circulation [16]. Additionally, ucMGP was recently purified from plasma of healthy subjects as a mature $11 \mathrm{kD}$ protein [17]. This suggests that the majority of healthy adults is subclinically vitamin $\mathrm{K}$ deficient, resulting in a sub-optimal activity of extra-hepatic vitamin K-dependent proteins. 
Indeed, it was described that women with calcified atherosclerotic lesions had lower dietary vitamin $\mathrm{K}$ intake than women without calcifications [18]. In a large follow-up study it was found that vitamin $\mathrm{K}_{2}$ intake was inversely associated with severe aortic calcification in asymptomatic subjects [19]. Additionally, the risk of coronary heart disease mortality was significantly reduced in the upper tertile of vitamin $\mathrm{K}_{2}$ intake. Our recently developed competitive enzyme-linked immunosorbent assay (ELISA) measuring total ucMGP ( $t$ ucMGP) provides a way to investigate the correlation between systemic t-ucMGP levels and vascular calcification, as described in this paper.

Impaired expression of MGP results in arterial calcification, as was demonstrated in transgenic MGP-deficient mice which developed extensive calcifications of the main arteries followed by rupture of the aorta a few weeks after birth [20]. The arterial mineralization phenotype of these mice has been rescued after restoration of MGP expression locally in arteries, however not after systemically raising circulating levels of MGP [21]. This emphasizes the function of MGP as a local inhibitor of vascular calcification, with circulating $t$ ucMGP levels possibly reflecting the status of calcification in the vessel wall [15].

The t-ucMGP assay specifically recognizes ucMGP, independent of its degree of phosphorylation. Using this assay we found that the serum t-ucMGP levels in HD and calciphylaxis patients as well as in patients with cardiovascular disease are lower than those in a healthy reference population [17]. We have previously demonstrated the massive accumulation of ucMGP at sites of arterial calcification [22], suggesting that - most probably through its negatively charged phosphoserine residues - MGP binds to calcified tissue independent of its Gla residues. This local tissue accumulation may be the reason for a diminished systemic release of ucMGP species into the circulation and may well explain the relatively low serum t-ucMGP levels in patients with pronounced vascular calcification.

A high coronary artery calcification (CAC) score as assessed with computed tomography is associated with the severity of coronary artery disease and the presence of significant, stenotic lesions [23] as well with increased mortality in HD patients [24]. To investigate whether circulating ucMGP may serve as a non-invasive biomarker for arterial calcification and - consequently - for cardiovascular disease in HD patients, we evaluated the relationship between serum t-ucMGP levels and the extent of CAC as measured with multislice computed tomography (MSCT) scanning. 


\section{Total uncarboxylated Matrix Gla-protein (t-ucMGP) \\ is associated with coronary artery calcification in \\ hemodialysis patients}

\section{Methods}

\section{$\underline{\text { Subjects }}$}

We invited patients undergoing HD treatment at three dialysis centers to participate in this cross-sectional study. Patients with end-stage renal disease (CKD stage V) undergoing regular HD were eligible, irrespective of age, gender, the underlying primary renal disease, the presence of CVD, or traditional atherogenic risk factors. Exclusion criteria were a history of kidney transplantation, current bypass surgery, current treatment with oral anticoagulants, and atrial fibrillation. Complete data and blood samples were acquired from 40 consecutive patients. Data were obtained on demographic, clinical, and dialysisspecific characteristics during interview, and physical examination as well as from review from patient's files. Additionally, all HD patients underwent MSCT scanning to measure the extent of CAC.

The reference population consists of two groups: 48 apparently healthy subjects aged 60 years or more, who were recruited from the general Maastricht population and 21 patients with rheumatoid arthritis (RA) from a tertiary RA referral center in Aachen. The subjects of the first reference group, 19 men and 29 women, had no overt history of cardiovascular disease and were described previously [17]; MSCT scanning was not available for these subjects. The second reference group consisted of 1 male and 20 female RA patients in whom MSCT scanning excluded the presence of CAC and aortic valve calcification (CAC score at both anatomical sites 0 ). Informed consent was obtained from all participants and the study was approved by the local Medical Ethics Committees.

\section{Biochemical measurements}

Venous blood samples from the HD patients were taken at the start of HD after a long dialysis interval. Serum from all participants was prepared by standard centrifugation and stored at $-80^{\circ} \mathrm{C}$ until testing.

The method for t-ucMGP measurement was a competitive enzyme-linked immunosorbent assay (ELISA) using a monoclonal antibody (mAb-ucMGP) which was raised against the uncarboxylated human MGP Gla-domain (MGP sequence 35-49; VitaK BV, Maastricht, The Netherlands) $[17,25]$. The intra-assay and inter-assay coefficients of variation (CV) were $8.9 \%$ and $11.4 \%$ respectively. The specificity of the t-ucMGP assay has been demonstrated elsewhere by testing of mAb-ucMGP for its cross-reaction with synthetic peptides homologous to the uncarboxylated and carboxylated aminoacid sequences in human MGP (ucMGP ${ }^{35-54}$ and $\mathrm{CMGP}^{35-54}$, respectively) and synthetic full length MGP (either ucMGP or CMGP) [17]. 
These polypeptides were used in competitive inhibition experiments with the biotinylated ucMGP tracer (ucMGP ${ }^{35-54}$ ). Both $\mathrm{ucMGP}^{35-54}$ and full length ucMGP were competitive inhibitors in the assay, whereas CMGP ${ }^{35-54}$ and full length CMGP were not. Additionally, immunoprecipitation was performed in plasma using the mAb-ucMGP. Identification of the immunoprecipitated product on Western blot analysis showed a single band at $11 \mathrm{kD}$ and analysis of the band on MALDI-TOF/TOF revealed UCMGP as protein identity in the immunoprecipitated product [17].

Calcium (Ca), phosphate (P), hemoglobin ( $\mathrm{Hb})$, and alkaline phosphatase (AP) were measured using standard laboratory techniques. Serum analysis for high-sensitivity C-reactive protein (hs-CRP) was performed by means of particle enhanced immunonephelometry using standard CardioPhase hsCRP for BNII (Dade Behring Holding GmbH, Liederbach, Germany), with a detection limit of $0.1 \mathrm{mg} / \mathrm{L}$ and precision of $0.1 \mathrm{mg} / \mathrm{L}$. CRPI or CRPII assay protocols were used when appropriate. Interday precision controls revealed CV's below 9\%; testing for precision with a 20 -fold repetitive measurement of a single sample showed a CV of $7 \%$ [26]. Levels of intact parathyroid hormone (iPTH) were measured with the Roche's Elecsys Intact PTH immunochemiluminescent assay (Hoffmann-La Roche, Basel, Switzerland).

\section{Imaging procedure}

All MSCT examinations of the HD patients and RA patients were performed with a 16-slice MSCT scanner (SOMATOM, Sensation 16, Siemens, Forchheim, Germany). Scan parameters included a collimation of $12 \times 0.75 \mathrm{~mm}$, a rotation time of $420 \mathrm{~ms}$, a table feed of 3.4 $\mathrm{mm}$ per rotation, a tube voltage of $120 \mathrm{kV}$ and an effective tube current time product of $150 \mathrm{mAseff}$. For ECG-synchronization retrospective ECG-gating was applied. Axial images were reconstructed in mid-diastole at $60 \%$ of the RR-interval with an effective slice thickness of $3 \mathrm{~mm}$ and a reconstruction increment of $2 \mathrm{~mm}$. A dedicated convolution kernel (B35f) [27], a field of view of $180 \times 180 \mathrm{~mm}^{2}$ and a matrix of $512 \times 512$ were applied. All MSCT images were assessed by two radiologists with several years of training in cardiac imaging. A consensus reading was performed with both readers being blinded to all patient data. Image analysis was performed on a separate computer workstation (Leonardo, Siemens, Forchheim, Germany) equipped with a dedicated software tool for calcium scoring (Calcium Scoring CT, Siemens, Forchheim, Germany). CAC scores were calculated according to the method originally described by Agatston [28].

\section{Statistical analysis}

Continuous variables are presented as median and range or mean \pm standard deviation (SD). The distribution of variables was evaluated with frequency histograms and normal plots. 


\section{Total uncarboxylated Matrix Gla-protein (t-ucMGP) \\ is associated with coronary artery calcification in \\ hemodialysis patients}

Differences between the patient population and the reference groups were analyzed by independent T-tests and Mann-Whitney tests (for normally and non-normally distributed continuous variables, respectively). The HD patient population was divided into tertiles of the CAC score which resulted in three groups with CAC scores of $\leq 103.0,103.1$ to 600.0, and $\geq 600.1$. Differences in clinical and biochemical characteristics between the three HD patient groups were analyzed by Tukey post hoc testing and Kruskal-Wallis test (for normally and non-normally distributed continuous variables, respectively) and Fisher's exact test (for categorical variables).

Bivariate correlations between CAC scores, t-ucMGP, and other variables were performed using the Pearson correlation coefficient for normally distributed data and Spearman correlation coefficient for non-normally distributed data. Multivariate regression analysis was performed to investigate whether CAC was related to serum t-ucMGP levels in HD patients, simultaneously adjusting for all variables in the model; the dependent variable was logtransformed CAC score. Log-transformation was applied after adding a constant of 10, which resulted in normal distribution of CAC score, necessary for multivariate regression analysis. The independent variables were t-ucMGP, age, dialysis vintage, and hs-CRP. Significant colinearity was ruled out by calculating variance inflation factor for each independent variable. A pvalue of $<0.05$ was considered to be statistically significant; reported $p$ values are based on two-tailed tests of statistical significance. All statistical analyses were conducted using SPSS version 12.0 for Windows (SPSS Corp, Chicago, IL, USA). 


\section{Results}

\section{Subject characteristics}

The reference population consisted of two groups, apparently healthy subjects and RA patients in whom the presence of CAC or aortic valve calcification was excluded by MSCT. The healthy reference group was free from cardiovascular disease, hypertension, and diabetes and did not use medication, as described previously [17]. The median age of these subjects (67 years, range 60-90 years) was comparable with that of the HD patient population (67 years, range $36-87$ years; $p=0.519$ ). The RA reference group consisted of 21 RA patients with a median age of 54 years (range 31 to 81 years), which was significantly lower than the median age of the HD patient population $(p<0.001)$. The median duration of the RA was 16 years (range 7-32 years). A history of cardiovascular disease was present in 5 RA patients (23.8\%), 1 patient had diabetes (4.8\%), and 2 patients $(9.5 \%)$ were current and 4 patients (19.0\%) were former smokers, respectively. Median blood pressure in the RA patients was $125 / 80 \mathrm{~mm} \mathrm{Hg}$.

Demographic, clinical, and biochemical characteristics of the HD patient population are listed in Table 1, with HD patients stratified according to tertiles of the CAC score. Subjects in the intermediate CAC tertile was significantly older than the subjects in the lowest CAC tertile $(p=0.039)$, however not as compared to the highest CAC tertile $(p=0.791)$. There was no significant age difference between subjects in the lowest and the highest CAC tertiles $(p=0.163)$. For the remaining demographic, clinical, and biochemical characteristics (Table 1), there were no significant differences between the tertiles. 


\section{Total uncarboxylated Matrix Gla-protein (t-ucMGP) is associated with coronary artery calcification in hemodialysis patients}

Table 1. Characteristics of HD patients stratified according to tertiles of the CAC score.

\begin{tabular}{|c|c|c|c|c|}
\hline & $\begin{array}{l}\text { CAC score } \\
\leq 103.0\end{array}$ & $\begin{array}{l}\text { CAC score } \\
103.1-600.0\end{array}$ & $\begin{array}{l}\text { CAC score } \\
\geq 600.1\end{array}$ & Total population \\
\hline $\mathrm{N}$ & 13 & 14 & 13 & 40 \\
\hline \multicolumn{5}{|l|}{ Demographic and clinical characteristics } \\
\hline Age (years) & $63(36-87)$ & $70(57-83) *$ & $68(52-79)$ & $67(36-87)$ \\
\hline Sex (male/female ratio) & $3 / 10$ & $6 / 8$ & $8 / 5$ & $17 / 23$ \\
\hline Dialysis vintage (months) & $44.9(4.0-113.5)$ & $30.4(8.2-109.4)$ & $33.8(5.4-289.5)$ & $34.3(4.0-289.5)$ \\
\hline \multicolumn{5}{|l|}{ Presumed cause of ESRD } \\
\hline \multicolumn{5}{|l|}{ (number of patients) } \\
\hline Glomerulonefritis, Vasculitis & 3 & 4 & 2 & 9 \\
\hline Tubulointerstitial disease & 2 & 1 & 1 & 4 \\
\hline Amyloid nephropathy & 3 & 0 & 0 & 3 \\
\hline Vascular disease, Hypertension & 1 & 5 & 2 & 8 \\
\hline Hereditary disease, Cystic disease & 4 & 1 & 3 & 8 \\
\hline Diabetic kidney disease & 0 & 0 & 3 & 3 \\
\hline Miscellaneous & 0 & 3 & 2 & 5 \\
\hline Hypertension (number of patients) & 12 & 13 & 1 & 38 \\
\hline Diabetes (number of patients) & 1 & 2 & 3 & 6 \\
\hline Current smoking (number of patients) & 1 & 2 & 4 & 7 \\
\hline BMI $\left(\mathrm{kg} / \mathrm{m}^{2}\right)$ & $25.6(18.4-53.3)$ & $24.1(17.3-31.4)$ & $23.9(19.1-36.6)$ & $24.4(17.3-53.3)$ \\
\hline \multicolumn{5}{|l|}{ History of cardiovascular disease } \\
\hline (number of patients) & 2 & 6 & 7 & 15 \\
\hline CAD & 2 & 5 & 5 & 12 \\
\hline CVD & 1 & 1 & 3 & 4 \\
\hline PAD & 1 & 2 & 3 & 6 \\
\hline \multicolumn{5}{|l|}{ History of parathyroidectomy } \\
\hline (number of patients) & 0 & 1 & 0 & 1 \\
\hline Vitamin D supplementation & 7 & 12 & 8 & 27 \\
\hline Use of oral PB & 13 & 12 & 11 & 31 \\
\hline Ca-containing PB & 8 & 12 & 11 & 31 \\
\hline Aluminum-containing PB & 2 & 6 & 3 & 11 \\
\hline Sevelamer & 3 & 0 & 1 & 4 \\
\hline \multicolumn{5}{|l|}{ Biochemical characteristics } \\
\hline $\mathrm{Ca}(\mathrm{mmol} / \mathrm{L})$ & $2.3(1.4-2.7)$ & $2.4(2.1-3.0)$ & $2.5(2.1-2.8)$ & $2.4(1.4-3.0)$ \\
\hline $\mathrm{P}(\mathrm{mmol} / \mathrm{L})$ & $1.8(1.0-2.5)$ & $1.4(1.1-2.5)$ & $1.8(1.3-2.6)$ & $1.7(1.0-2.6)$ \\
\hline $\left.\mathrm{Ca} \times \mathrm{P}(\mathrm{nmol})^{2} / \mathrm{L}^{2}\right)$ & $3.9(2.3-6.6)$ & $3.3(2.7-5.7)$ & $4.2(2.9-7.3)$ & $4.1(2.3-7.3)$ \\
\hline hs-CRP (mg/L) & $2.5(0.3-18.9)$ & $5.6(1.3-24.8)$ & $7.2(0.2-21.4)$ & $4.8(0.2-24.8)$ \\
\hline Hemoglobin (mg/dL) & $104(88-124)$ & $109(95-144)$ & $106(95-156)$ & $105(88-156)$ \\
\hline $\mathrm{AP}(\mathrm{U} / \mathrm{L})$ & $58(41-232)$ & $52(40-252)$ & $51(34-217)$ & $54(34-252)$ \\
\hline iPTH (pmol/L) & $7.0(1.1-276)$ & $9.6(1.2-116)$ & $10.5(0.7-164)$ & $9.6(0.7-276)$ \\
\hline
\end{tabular}

Demographic, clinical, and biochemical characteristics of patients stratified according to tertiles of the CAC score: $C A C$ score $\leq 130.0,103.1$ to 600.0 and $\geq 600.1$. Data are presented as median (range). *: The intermediate CAC tertile was significantly older as compared to the lowest CAC tertile $(p=0.039)$. Otherwise, there were no significant differences between the CAC tertiles. Abbreviations used: CAC, coronary artery calcification; ESRD, end-stage renal disease; $\mathrm{BMI}$, body mass index; $\mathrm{CAD}$, coronary artery disease, defined as history of myocardial infarction, angina pectoris or evidence of obstructive disease by angiography; CVD, cerebrovascular disease, defined as a history of thrombotic stroke or transient ischemic attack; PAD, peripheral artery disease, defined as a history of claudication or lower extremity revascularization; PB, phosphate binders; Ca, calcium; P, phosphate; hs-CRP, high-sensitivity C-reactive protein; AP, alkaline phosphatase; iPTH, intact parathyroid hormone. 


\section{Circulating ucMGP}

The mean t-ucMGP levels of the apparently healthy subjects and RA patients without CAC were $441 \pm 97 \mathrm{nmol} / \mathrm{L}$ and $560 \pm 140 \mathrm{nmol} / \mathrm{L}$, respectively. The mean $\mathrm{t}$-uCMGP level of the HD patient population was $193 \pm 65 \mathrm{nmol} / \mathrm{L}$, with levels ranging from 60 to $352 \mathrm{nmol} / \mathrm{L}$ (Figure 1). The HD patient population had significantly lower circulating t-ucMGP levels in comparison with both reference groups $(p<0.001)$. Moreover, the serum $t$-ucMGP levels of the healthy subjects were significantly lower compared than those in RA patients $(p<0.001)$.

Figure 1: Serum t-ucMGP levels of the RA reference group $(n=21)$, the healthy reference group $(n=48)$, and the HD patient population $(n=40)$.

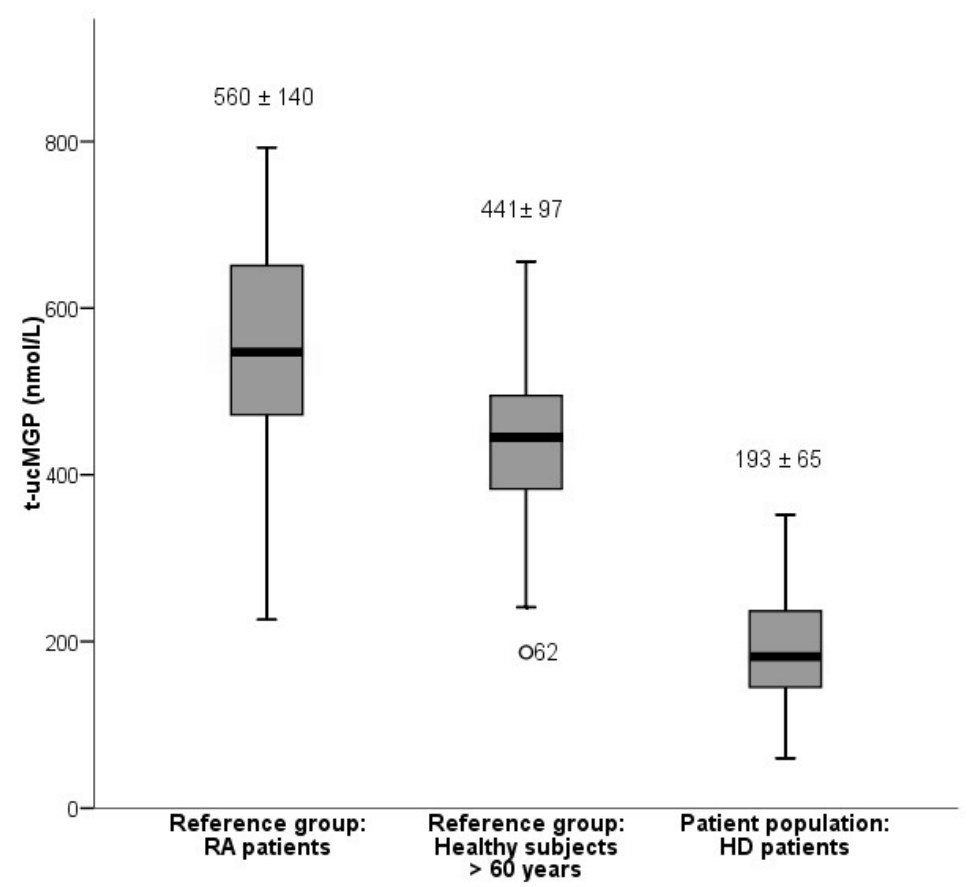

Circulating t-ucMGP levels were significantly lower in HD patients than in RA patients $(\mathrm{p}<0.001)$ and apparently healthy subjects aged 60 years or more $(p<0.001)$. One outlier, subject number 62 (depicted as $\circ 62$ ), was present in the healthy reference group. This subject (age 66 years) had a t-ucMGP level of $187 \mathrm{nmol} / \mathrm{L}$. Box diagrams represent median (horizontal line), the $75^{\text {th }}$ percentile (upper limit of the box) and the $25^{\text {th }}$ percentile (lower limit of the box). Whiskers show the range of values, with exception of outliers and extremes. Per box the mean $\pm S D$ ucMGP level is presented. Abbreviations used: RA, rheumatoid arthritis; HD, hemodialysis. 


\section{Total uncarboxylated Matrix Gla-protein (t-ucMGP) \\ is associated with coronary artery calcification in hemodialysis patients}

No significant correlations were observed between t-ucMGP levels and any demographic, clinical, or biochemical characteristic in the HD patient population. There was no difference in t-ucMGP levels between men and women; this was also found in both reference groups. Figure 2 shows the serum t-ucMGP levels of HD patients stratified according to tertiles of the CAC score; t-ucMGP levels were significantly lower in patients in the intermediate $(174 \pm 46 \mathrm{nmol} / \mathrm{L})$ and highest CAC tertile $(171 \pm 66 \mathrm{nmol} / \mathrm{L})$ in comparison with patients in the lowest CAC tertile $(237 \pm 66 \mathrm{nmol} / \mathrm{L} ; \mathrm{p}=0.022$ and $\mathrm{p}=0.021$, respectively).

Figure 2: Serum t-ucMGP levels of patients stratified according to tertiles of the CAC score $(n=40)$.

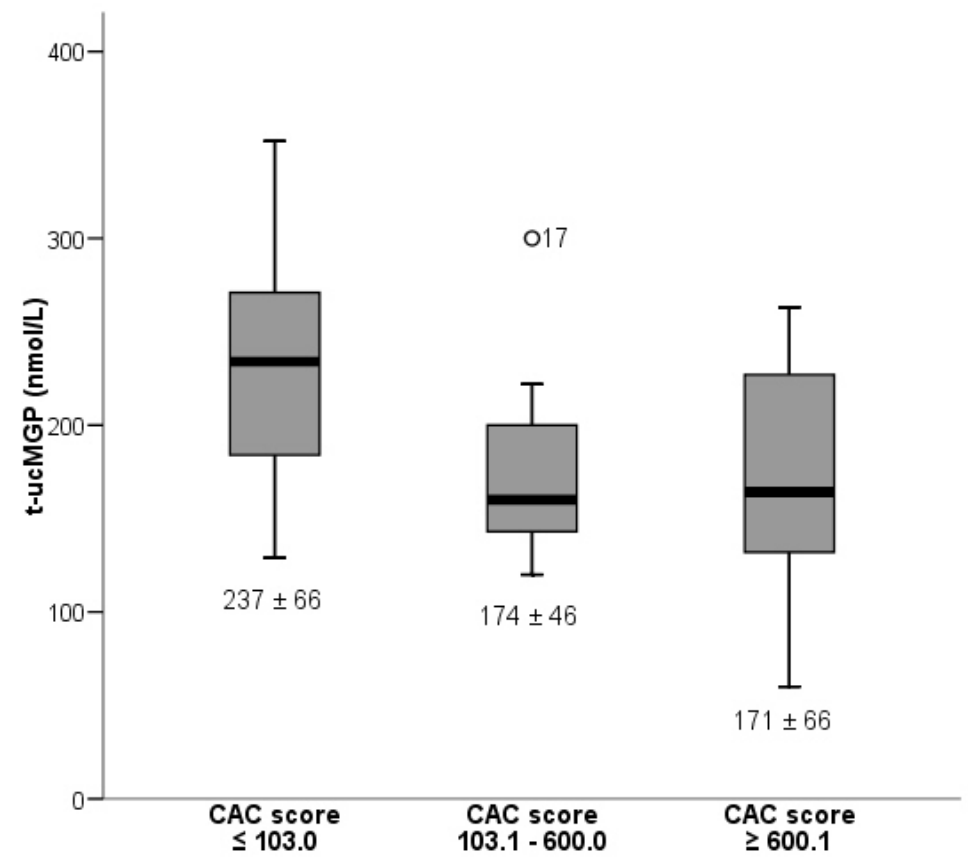

Circulating t-ucMGP levels in HD patients in the intermediate and highest CAC tertiles were significantly lower than in patients in the lowest $C A C$ tertile $(p=0.022$ and $p=0.021$ respectively). One outlier, patient number 17 (depicted as $\circ 17$ ), was present in the intermediate CAC tertile. This HD patient (age 60 years, dialysis vintage 21 months) had a circulating ucMGP level of $300 \mathrm{nmol} / \mathrm{L}$. Box diagrams represent median (horizontal line), the $75^{\text {th }}$ percentile (upper limit of the box) and the $25^{\text {th }}$ percentile (lower limit of the box). For further details, see the legend of Figure 1. Abbreviation used: CAC, coronary artery calcification. 


\section{t-ucMGP as a predictor for CAC in HD patients}

A high prevalence of CAC was a major finding in our patient population, with 18 out of 40 patients (45\%) having a CAC score of 400 or more, indicating extensive atherosclerotic plaque burden [29]. The median CAC score of the total HD patient population was 308.0 (range 0-2928). Serum t-ucMGP levels were inversely correlated with CAC scores $(r=-0.414, p=0.009)$; the only other factor that correlated with CAC scores was hs-CRP ( $r=0.404, p=0.011$; Table 2). In contrast, CAC scores did not correlate with any of the other clinical and biochemical characteristics (depicted in Table 2 for the main clinical and biochemical parameters). In a multivariate regression analysis t-ucMGP levels retained a strong and significant association with CAC scores ( $\beta=-0.004, p=0.02)$ after adjustment for age, dialysis vintage, and hs-CRP levels (Table 2).

Table 2. Coronary artery calcification correlations $(n=40)$.

\begin{tabular}{llll}
\hline Bivariate & $\mathbf{r}$ & $\mathbf{p}$ & \\
Age & 0.29 & 0.072 & \\
Dialysis vintage & 0.03 & 0.849 & \\
BMI & -0.17 & 0.295 & \\
Ca & 0.27 & 0.098 & \\
P & 0.01 & 0.964 & \\
CaxP & 0.06 & 0.713 & \\
hs-CRP & 0.40 & 0.011 & \\
AP & -0.047 & 0.781 & \\
iPTH & 0.068 & 0.687 & $\mathbf{9 5 \%} \mathbf{C l}$ \\
ucMGP & -0.41 & 0.009 & 0.007 to -0.001 \\
Multivariate & $\mathbf{\beta}$ coefficient & $\mathbf{p}$ & 0.001 to 0.032 \\
uCMGP & 0.004 & 0.017 & 0.002 to 0.006 \\
Age & 0.016 & 0.056 & 0.003 to 0.067 \\
Dialysis vintage & 0.002 & 0.382 & \\
hs-CRP & 0.035 & 0.031 & \\
\hline
\end{tabular}

For bivariate analysis (bivariate) the Spearman rank correlation coefficient was calculated. Circulating t-ucMGP levels and hs-CRP were significantly correlated with CAC scores; CAC scores did not correlate with any of the other clinical and biochemical parameters. In multivariate regression analysis (multivariate) t-ucMGP levels retained a strong association with (log-transformed) CAC scores while adjusting for age, dialysis vintage and hsCRP-levels. The overall model $p$ value was 0.002 , the model $R^{2}$ was 0.377 (adjusted $R^{2}=0.306$ ); the $\beta$ regression coefficient was calculated per $1 \mathrm{nmol} / \mathrm{L}$ t-ucMGP. Abbreviations used: BMI, body mass index; Ca, calcium; P, phosphate; AP, alkaline phosphatase; $\mathrm{PTH}$, intact parathyroid hormone; hs-CRP, high-sensitivity C-reactive protein. 


\section{Total uncarboxylated Matrix Gla-protein (t-ucMGP) \\ is associated with coronary artery calcification in \\ hemodialysis patients}

\section{Discussion}

The present study establishes serum levels of t-ucMGP as a possible novel biomarker for CAC in HD patients. HD patients had significantly lower levels of t-ucMGP than healthy subjects of the same age and patients without CAC. Additionally, HD patients in the intermediate and highest tertiles of the CAC score had significantly lowert-ucMGP levels in comparison with patients in the lowest CAC tertile. The inverse correlation between serum t-ucMGP and CAC scores was confirmed by multivariate regression analysis and persisted after adjustment for age, dialysis vintage, and hs-CRP - three well established predictors of CAC in HD patients [5, 6, 8-10, 30].

We previously found significantly lower t-ucMGP levels in several patient populations with a high vascular calcification burden, including HD patients and calciphylaxis patients [17]. The present study underlines and extends these findings and shows that t-ucMGP serum measurements may not only be used for intergroup comparisons of arterial calcification, but also help estimating the individual extent of CAC in a certain patient population. It is noteworthy that the mean $\pm S D$ t-ucMGP level of $140 \pm 55 \mathrm{nmol} / \mathrm{L}$ previously found in patients with calciphylaxis - a condition characterized by widespread arteriolar calcification - is even lower than that of HD patients with the highest CAC scores in the present study [17].

Defining t-ucMGP as a sensitive indicator for the presence of vascular calcification might also be the explanation for the surprising finding of lower circulating t-ucMGP levels in the apparently healthy subjects compared to the RA patients. Only in the RA reference group, the presence of CAC was truly excluded, while clinically unapparent CAC might be present in the presumably healthy subjects who were aged 60 years or more. Indeed, several studies have described median CAC scores $>100$ in elderly subjects without overt signs of atherosclerosis, especially in elderly men [31-33]. We speculate that subclinical arterial disease might contribute to the lower t-ucMGP levels in the "healthy" reference group. At the present time it cannot be determined, whether RA itself, or a consecutive treatment influences t-ucMGP levels in the RA reference group.

The design of the present study is cross-sectional and therefore we cannot establish a cause-and-effect relation between low t-ucMGP levels and high CAC scores. However, it does allow for assessment of t-ucMGP as a biomarker for CAC. The number of HD patients is limited and therefore we are limited with respect to full adjustment for all possible confounders in our multivariate analysis. Nevertheless, the present patient population is well characterized and by including age, dialysis vintage, and hs-CRP levels we could adjust at least for three of the most important potential confounders regarding CAC development in ESRD patients. 
ucMGP species have a high affinity to areas of calcification in the vessel wall. In rats treated with warfarin, Price et al. demonstrated that MGP accumulates at sites of calcification in its non-carboxylated form [34]. Sweatt et al. reported elevated concentrations of poorly carboxylated MGP in the aortic wall of aging rats [35]. Moreover, with immunohistochemical staining based on conformation-specific antibodies recognizing ucMGP and CMGP, we demonstrated that ucMGP rather than CMGP species are present at sites of arterial calcification [22]. Therefore, our working hypothesis is that the presence of Gla residues is not a requirement for the binding of MGP to sites of calcification. Indeed, thermal decarboxylation of MGP in vitro did not affect its ability to bind hydroxyapatite from serum [36].

Phosphorylation of MGP results in the presence of three phosphoserine residues in the Nterminal domain. These negatively-charged phosphate groups must be expected to contribute significantly to the calcium-binding capacity of MGP. We examined the role of phosphoserine-residues in a cell model of calcification [37]. This model consisted of VSMC exposed to high extracellular calcium concentrations. The synthetic MGP peptides PMGP ${ }^{3-}$ ${ }^{15}$ and $\mathrm{dpMGP} \mathrm{P}^{3-15}$, which were used in the experiments, contain three serine residues that are either phosphorylated or non-phosphorylated, respectively. These peptides do not contain Gla residues. After addition of the (biotinylated) peptides to the calcifying VSMC, staining revealed that $\mathrm{pMGP}^{3-15}$ specifically adhered to vesicular structures derived from the (calcifying) VSMC. These vesicles were presumably matrix vesicles, which are thought to precede calcification [38]. In contrast, dpMGP ${ }^{3-15}$ did not bind to either the cell surface or the vesicles. $\mathrm{PMGP}^{3-15}$ was also able to inhibit the calcification process, whereas dpMGP ${ }^{3-15}$ had no measurable effect. These results indicate that MGP containing phosphoserine residues, but no Gla residues, is able to bind to (initial) sites of calcification.

Since ucMGP species in the vascular tissue have a strong association with areas of calcification, circulating t-ucMGP levels could be particularly suited to reflect this degree of this calcification. A low systemic t-ucMGP level in patients with a high vascular calcification burden has been a consistent finding $[17,25,39]$. The low t-ucMGP levels in these patients can be explained by the accumulation of ucMGP species in areas of calcification, which may cause a diminished release of ucMGP species into the circulation. This accumulation is most probably due to binding of MGP's negatively charged phosphoserine residues to vascular calcifications. It is therefore likely that the majority of circulating ucMGP species is phosphorylated. Indeed, we have found no correlation between circulating ucMGP levels and non-phosphorylated MGP levels in dialysis patients ( $r=-0.144, p=0.38$; data not published). 


\section{Total uncarboxylated Matrix Gla-protein (t-ucMGP) \\ is associated with coronary artery calcification in \\ hemodialysis patients}

Since phosphorylation is often associated with cellular secretion, it is at least feasible that in the diseased vessel MGP phosphorylation is hampered, leading to decreased circulating levels. This could be an alternative explanation for the low circulating t-ucMGP levels in patients with vascular calcifications.

Recently, we developed a new sandwich MGP ELISA specifically measuring desphosphouncarboxylated MGP (dp-ucMGP) [15]. Since strong calcium-binding groups such as phosphoserine- or Gla-residues are absent in this molecule, we expect that dp-ucMGP will be readily set free into the circulation independent of present vascular calcification. This assay would therefore be suited to assess and follow-up vascular vitamin $\mathrm{K}$ status (e.g. when vitamin $\mathrm{K}$ supplementation or vitamin $\mathrm{K}$ antagonists are used). Preliminary data showed that dp-ucMGP is present in the circulation of healthy subjects [15]. High dpucMGP levels were found in subjects receiving vitamin $\mathrm{K}$ antagonists and low levels in subjects receiving vitamin $\mathrm{K}$ supplementation. The data presently available strongly suggest that dp-ucMGP reflects vascular vitamin $\mathrm{K}$ status. In contrast, $\mathrm{t}$-ucMGP levels as described in this paper seem to be relatively insensitive for vitamin $\mathrm{K}$ status (data not published) but are rather sensitive for the presence of arterial calcification. Full details of the dp-ucMGP assay will be published elsewhere.

The dialysis population exhibits a significant amount of vascular calcification that is associated with an increased risk of cardiovascular morbidity and mortality. According to the present data it can be hypothesized that measuring merely calcification promoters (e.g. serum calcium or serum phosphorus) does not give sufficient consideration to the role of calcification inhibitors in the process of vascular calcification. Based on the present data, circulating t-ucMGP could contribute substantially to the identification of those patients with high probability of cardiovascular calcification. Further studies need to be performed in order to clarify whether low t-ucMGP levels may eventually be used to identify those patients who should undergo a more thorough diagnostic cardiac work-up (e.g. noninvasive ischemia testing or coronary angiography).

In conclusion, the current study is the first analysis of circulating t-ucMGP levels in a well characterized population of adult HD patients and demonstrates that t-ucMGP is significantly and inversely correlated with CAC as assessed by MSCT. 


\section{Acknowledgements}

This work was supported by a grant from GENZYME. The authors thank Dr. A. Kester for his statistical advice and Mrs. M.-L. Boumans for her excellent work in laboratory analysis. The authors also wish to thank Prof. Dr. J. Rosing for critical reading the manuscript.

\section{References}

1. US Renal Data System. USRDS 1998 annual data report. Bethesda: National Institute of Diabetes and Kidney Diseases 1999: 63-90.

2. US Renal Data System. USRDS 1998 annual data report. Bethesda: National Institute of Diabetes and Kidney Diseases 1999: 79-90.

3. Shanahan, C.M., et al., Medial localization of mineralization-regulating proteins in association with Monckeberg's sclerosis: evidence for smooth muscle cell-mediated vascular calcification. Circulation, 1999. 100(21): p. 2168-76.

4. Lehto, S., et al., Medial artery calcification. A neglected harbinger of cardiovascular complications in noninsulin-dependent diabetes mellitus. Arterioscler Thromb Vasc Biol, 1996. 16(8): p. 978-83.

5. Blacher, J., et al., Arterial calcifications, arterial stiffness, and cardiovascular risk in end-stage renal disease. Hypertension, 2001. 38(4): p. 938-42.

6. London, G.M., et al., Arterial media calcification in end-stage renal disease: impact on all-cause and cardiovascular mortality. Nephrol Dial Transplant, 2003. 18(9): p. 1731-40.

7. London, G.M., et al., Arterial structure and function in end-stage renal disease. Nephrol Dial Transplant, 2002. 17(10): p. 1713-24.

8. Guerin, A.P., et al., Arterial stiffening and vascular calcifications in end-stage renal disease. Nephrol Dial Transplant, 2000. 15(7): p. 1014-21.

9. Raggi, P., et al., Cardiac calcification in adult hemodialysis patients. A link between end-stage renal disease and cardiovascular disease? J Am Coll Cardiol, 2002. 39(4): p. 695-701.

10. McCullough, P.A., et al., Determinants of coronary vascular calcification in patients with chronic kidney disease and end-stage renal disease: a systematic review. J Nephrol, 2004. 17(2): p. 205-15.

11. Luo, G., et al., The matrix Gla protein gene is a marker of the chondrogenesis cell lineage during mouse development. J Bone Miner Res, 1995. 10(2): p. 325-34.

12. Wallin, R., D. Cain, and D.C. Sane, Matrix Gla protein synthesis and gamma-carboxylation in the aortic vessel wall and proliferating vascular smooth muscle cells--a cell system which resembles the system in bone cells. Thromb Haemost, 1999. 82(6): p. 1764-7.

13. El-Maadawy, S., et al., Cartilage formation and calcification in arteries of mice lacking matrix Gla protein. Connect Tissue Res, 2003. 44 Suppl 1: p. 272-8.

14. Vermeer, C., Gamma-carboxyglutamate-containing proteins and the vitamin K-dependent carboxylase. Biochem J, 1990. 266(3): p. 625-36.

15. Schurgers, L.J., E.C.M. Cranenburg, and C. Vermeer, Matrix Gla-protein: The calcification inhibitor in need of vitamin K. Thromb Haemost, 2008. 100(4): p. 593-603.

16. Cranenburg, E.C., L.J. Schurgers, and C. Vermeer, Vitamin K: The coagulation vitamin that became omnipotent. Thromb Haemost, 2007. 98(1): p. 120-125.

17. Cranenburg, E.C., et al., The circulating inactive form of Matrix Gla Protein (ucMGP) as a biomarker for cardiovascular calcification J Vasc Res 2008. 45: p. 427-436.

18. Jie, K.S., et al., Vitamin K intake and osteocalcin levels in women with and without aortic atherosclerosis: a population-based study. Atherosclerosis, 1995. 116(1): p. 117-23. 


\section{Total uncarboxylated Matrix Gla-protein (t-ucMGP) \\ is associated with coronary artery calcification in \\ hemodialysis patients}

19. Geleijnse, J.M., et al., Dietary intake of menaquinone is associated with a reduced risk of coronary heart disease: the Rotterdam Study. J Nutr, 2004. 134(11): p. 3100-5.

20. Luo, G., et al., Spontaneous calcification of arteries and cartilage in mice lacking matrix GLA protein. Nature, 1997. 386(6620): p. 78-81.

21. Murshed, M., et al., Extracellular matrix mineralization is regulated locally; different roles of two glacontaining proteins. J Cell Biol, 2004. 165(5): p. 625-30.

22. Schurgers, L.J., et al., Novel conformation-specific antibodies against matrix gamma-carboxyglutamic acid (Gla) protein: undercarboxylated matrix Gla protein as marker for vascular calcification. Arterioscler Thromb Vasc Biol, 2005. 25(8): p. 1629-33.

23. Robinson, J., et al., Electron-Beam Computerized Tomography Correlates with Coronary Angiogram in Chronic Kidney Disease Patients. Am J Nephrol, 2007. 27(3): p. 247-52.

24. Block, G.A., et al., Mortality effect of coronary calcification and phosphate binder choice in incident hemodialysis patients. Kidney Int, 2007. 71(5): p. 438-41.

25. Hermans, M.M., et al., Undercarboxylated Matrix GLA Protein Levels Are Decreased in Dialysis Patients and Related to Parameters of Calcium-Phosphate Metabolism and Aortic Augmentation Index. Blood Purif, 2007. 25(5): p. 395-401.

26. Hermans, M.M., et al., Study on the relationship of serum fetuin-A concentration with aortic stiffness in patients on dialysis. Nephrol Dial Transplant, 2006. 21(5): p. 1293-9.

27. Mahnken, A.H., et al., Variation of the coronary calcium score depending on image reconstruction interval and scoring algorithm. Invest Radiol, 2002. 37(9): p. 496-502.

28. Agatston, A.S., et al., Quantification of coronary artery calcium using ultrafast computed tomography. J Am Coll Cardiol, 1990. 15(4): p. 827-32.

29. Rumberger, J.A., et al., Electron beam computed tomographic coronary calcium scanning: a review and guidelines for use in asymptomatic persons. Mayo Clin Proc, 1999. 74(3): p. 243-52.

30. Goodman, W.G., et al., Coronary-artery calcification in young adults with end-stage renal disease who are undergoing dialysis. N Engl J Med, 2000. 342(20): p. 1478-83.

31. Newman, A.B., et al., Coronary artery calcification in older adults to age 99: prevalence and risk factors. Circulation, 2001. 104(22): p. 2679-84.

32. Barrett-Connor, E., G.A. Laughlin, and C. Connor, Coronary artery calcium versus intima-media thickness as a measure of cardiovascular disease among asymptomatic adults (from the Rancho Bernardo Study). Am J Cardiol, 2007. 99(2): p. 227-31.

33. Fair, J.M., et al., Ethnic differences in coronary artery calcium in a healthy cohort aged 60 to 69 years. Am J Cardiol, 2007. 100(6): p. 981-5.

34. Price, P.A., S.A. Faus, and M.K. Williamson, Warfarin-induced artery calcification is accelerated by growth and vitamin D. Arterioscler Thromb Vasc Biol, 2000. 20(2): p. 317-27.

35. Sweatt, A., et al., Matrix Gla protein (MGP) and bone morphogenetic protein-2 in aortic calcified lesions of aging rats. J Thromb Haemost, 2003. 1(1): p. 178-85.

36. Price, P.A., S.A. Faus, and M.K. Williamson, Warfarin causes rapid calcification of the elastic lamellae in rat arteries and heart valves. Arterioscler Thromb Vasc Biol, 1998. 18(9): p. 1400-7.

37. Schurgers, L.J., et al., Post-translational modifications regulate matrix Gla protein function: importance for inhibition of vascular smooth muscle cell calcification. J Thromb Haemost, 2007. 5(12): p. 2503-11.

38. Proudfoot, D., et al., Apoptosis regulates human vascular calcification in vitro: evidence for initiation of vascular calcification by apoptotic bodies. Circ Res, 2000. 87(11): p. 1055-62.

39. Shroff, R.C., et al., The circulating calcification inhibitors, fetuin-A and osteoprotegerin, but not Matrix Gla protein, are associated with vascular stiffness and calcification in children on dialysis. Nephrol Dial Transplant, 2008. 23(10): p. 3263-71. 



\section{Chapter}

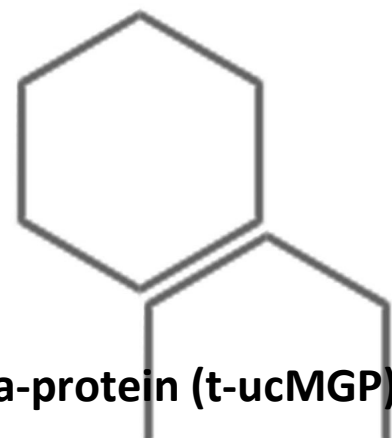

\section{is not associated with mortality in dialysis patients \\ Circulating total uncarboxylated Matrix Gla-protein (t-ucMGP}

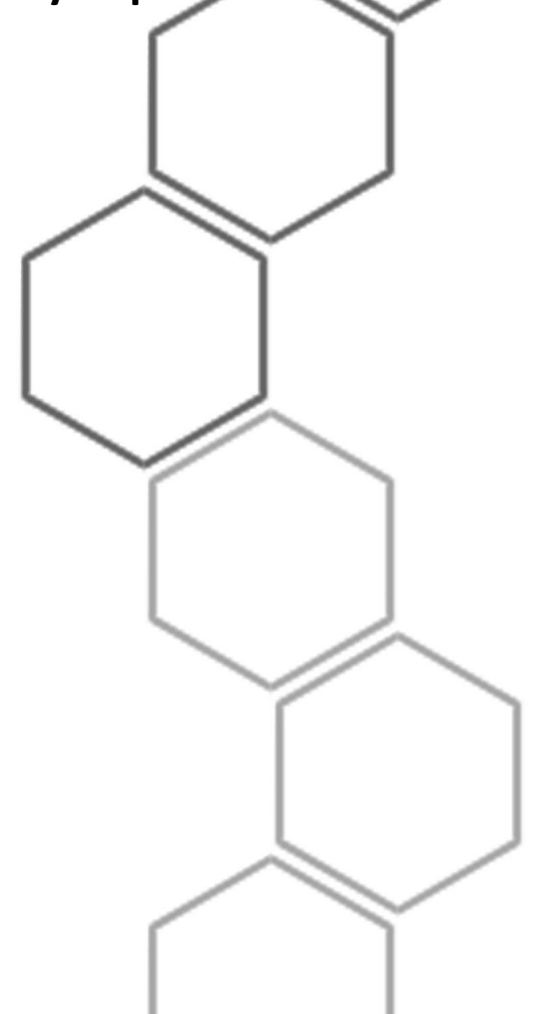

Ellen C.M. Cranenburg ${ }^{1}$, Marlies Noordzij ${ }^{2}$, Leon J. Schurgers ${ }^{1}$, Jeroen P. Kooman ${ }^{3}$, Marc M.H. Hermans ${ }^{4}$, Cees Vermeer ${ }^{1}$, Elisabeth W. Boeschoten ${ }^{5}$, Friedo W. Dekker ${ }^{6}$, and Marion Verduijn ${ }^{6}$ for the NECOSAD study group

${ }^{1}$ VitaK and Cardiovascular Research Institute Maastricht (CARIM), Maastricht, the Netherlands; ${ }^{2}$ Department of Medical Informatics, Academic Medical Center, University of Amsterdam, Amsterdam, the Netherlands; ${ }^{3}$ Department of Internal Medicine and Nephrology, Academic Hospital Maastricht, Maastricht, the Netherlands; ${ }^{4}$ Department of Internal Medicine , Viecuri medical center, Venlo, the Netherlands; ${ }^{5}$ Hans Mak Institute, Naarden, the Netherlands; ${ }^{6}$ Department of Clinical Epidemiology, Leiden University Medical Center, Leiden, the Netherlands. 


\begin{abstract}
Matrix $\mathrm{p}$-carboxyglutamate (Gla)-protein (MGP), a vitamin K-dependent protein, is an important local inhibitor of vascular calcification. Patients undergoing dialysis treatment are at high risk to develop vascular calcification, which results in significant morbidity and mortality. Vitamin K deficiency leads to synthesis of uncarboxylated MGP (UCMGP), which accumulates at sites of calcification in the arterial wall. Previous studies showed that circulating total ucMGP levels (t-ucMGP) were low in dialysis patients, and were inversely correlated with vascular calcification parameters. In the present study, we have investigated the predictive value of t-ucMGP for overall and cardiovascular mortality in incident dialysis patients.
\end{abstract}

Serum t-ucMGP levels were measured with a competitive enzyme-linked immunosorbent assay in 952 patients from NECOSAD, a large prospective multicenter Dutch cohort of incident hemodialysis (HD) and peritoneal dialysis (PD) patients. Patients were divided into tertiles according to t-ucMGP levels (high, medium, and low) to investigate whether there was a difference in mortality risk between the tertiles.

Almost all patients had t-ucMGP levels far below the normal range $(300-700 \mathrm{nmol} / \mathrm{L})$, with HD patients having significantly lower levels (mean $\pm S D ; 154 \pm 59 \mathrm{nmol} / \mathrm{L}$ ) compared to PD patients $(189 \pm 77 \mathrm{nmol} / \mathrm{L} ; \mathrm{p}<0.001)$. In the total study population, t-ucMGP levels inversely correlated with pulse pressure $(r=-0.157, p<0.001)$. No significant differences were found between the high, low, and medium t-ucMGP tertiles in overall, cardiovascular, and non-cardiovascular mortality.

Our results indicate that t-uCMGP is less suited for predicting mortality in this patient population, probably due to very low t-ucMGP levels in dialysis patients. 


\section{Circulating total-uncarboxylated Matrix \\ Gla-protein (t-ucMGP) is not associated with \\ mortality in dialysis patients}

\section{Introduction}

Mortality among dialysis patients is high, with expected life times ranging from one-fifth to half of those in the general population [1]. The presence and extent of vascular calcification are predictors of mortality, independent of traditional atherogenic risk factors in dialysis patients [2]. Clinically, vascular calcification may result in left ventricular hypertrophy and decreased coronary perfusion with increased risk of death [2-4].

The process of vascular calcification is an active, cell-mediated process in which both promoters and inhibitors play a role. An important inhibitor of vascular calcification present in the arterial wall is matrix $\gamma$-carboxyglutamate (Gla)-protein (MGP), which is synthesized by vascular smooth muscle cells. MGP can undergo two post-translational modifications: $y$-glutamate carboxylation and serine phosphorylation [5-7]. The oxidation of vitamin K-hydroquinone provides the energy for MGP carboxylation, and this results in the synthesis of carboxylated MGP (cMGP) [8]. CMGP is able to bind to calcium-crystals, and inhibit the osteogenic growth factor BMP-2 $[9,10]$; both mechanisms underlie the calcification-inhibitory activity of MGP [11-13]. In case of vitamin K deficiency, uncarboxylated MGP (ucMGP) will be secreted, which has been consistently found to accumulate at sites of calcification in the arterial wall [14-16].

Several enzyme-linked immunosorbent assays (ELISA) have been developed to measure circulating MGP levels. The first assay which was able to discriminate between CMGP and ucMGP, was the competitive ELISA detecting circulating total ucMGP (t-ucMGP), independent of phosphorylation state [17]. With this assay, low t-ucMGP levels have been found in patients with end-stage renal disease (ESRD) [17-19], which is consistent with the accumulation of ucMGP species in vascular tissue at sites of calcification [8]. In addition, an inverse correlation was found between circulating t-ucMGP levels and both the aortic augmentation index and the extent of coronary artery calcification in dialysis patients $[18,20]$.

In the present study, we evaluated the potential predictive value of circulating t-ucMGP for overall and cardiovascular (CV) mortality in dialysis patients, which has not been investigated previously. Serum t-ucMGP levels were therefore determined in a large, prospective, multicenter Dutch cohort study of incident hemodialysis (HD) and peritoneal dialysis (PD) patients. 


\section{Methods}

\section{Patients}

The Netherlands Cooperative Study on the Adequacy of Dialysis (NECOSAD) is a large prospective multicenter cohort study, in which ESRD patients were followed from the initiation of dialysis treatment until transplantation or death. All incident ESRD patients in 38 dialysis units in The Netherlands were consecutively invited to participate in the study. Patients had to be 18 years or older, with dialysis as their first renal replacement therapy. The study was approved by all local medical ethics committees and all patients gave informed consent before inclusion. For the present analysis, we included incident HD and PD patients who started on long-term dialysis treatment between 1997 and 2005 and who survived the first 3 months of dialysis. Patients were followed until transplantation, mortality, or the end of the study period (26 May 2009).

\section{Data collection}

Data on demographics, primary kidney disease, and comorbidity were collected 0-4 weeks before start of dialysis treatment. Data on residual renal function, biochemistry and dialysis characteristics were collected at 3 months after the start of dialysis.

Primary kidney disease and causes of death were classified according to the codes of the European Renal Association-Dialysis and Transplantation Association (ERA-EDTA) [21]. CV mortality was defined as any death attributed to myocardial ischemia and infarction, cardiac failure, cardiac arrest (cause unknown), cerebrovascular accident, fluid overload, hyper- and hypokalemia, haemorrhage from a ruptured aneurysm or mesenteric infarction. If the cause of death was uncertain, unknown or could not be determined, mortality was classified as cardiovascular. All other causes of death were classified as noncardiovascular.

Blood pressure measurements were performed at 3 months of dialysis. BP was measured before the start of a dialysis session and at the end of the same session in HD patients. In general, the measurements were performed with oscillometric devices incorporated in the dialysis machines.

Prevalent CV disease (CVD) was classified as the presence or history of ischemic heart disease, peripheral vascular disease, heart failure, and/or cerebrovascular disease. Comorbidity was defined according to the risk criteria of Khan et al. [22]. The Kahn index is a combination of age and comorbidity resulting in 3 risk groups: low, medium, and high. 


\section{Circulating total-uncarboxylated Matrix Gla-protein (t-ucMGP) is not associated with mortality in dialysis patients}

The nutritional status was scored on the standardized 7-point scale of the Subjective Global Assessment (SGA), which is based on the clinical judgement of the dialysis nurse. Malnourishment was defined as an SGA score of 5 or less.

Residual renal function was expressed as residual glomerular filtration rate (rGFR), calculated as the mean of creatinine and urea clearance adjusted for body surface area (in $\mathrm{ml} / \mathrm{min} / 1.73 \mathrm{~m}^{2}$ ). Dialysis dose, expressed as $\mathrm{K}_{\mathrm{t}} / V$ urea per week, was calculated as dialysis urea clearance divided by urea distribution volume (V) according to Watson et al. [23]. For HD patients, dialysis urea clearance was calculated by using a second-generation Daugirdas formula [24], and for PD patients peritoneal $\mathrm{K}_{\mathrm{t}} / V$ was calculated by using 24-h dialysate collection.

All laboratory analyses were performed in blood samples obtained 3 months after the initiation of dialysis treatment. In HD patients, blood samples were obtained just before the start of HD treatment after a short interval. Plasma calcium, phosphorus, and albumin were measured using standard laboratory techniques in the different centers. Calcium concentration ( $\mathrm{mmol} / \mathrm{l}$ ) was corrected for albumin concentration. Measurements of intact parathyroid hormone (iPTH) were performed by various first-generation immunometric iPTH-assays depending on the different participating centers. Measurements of highsensitivity C-reactive protein (hs-CRP) were performed by means of particle-enhanced immunonephelometry using a standard CardioPhase hsCRP for BNII (Dade Behring Holding $\mathrm{GmbH}$, Liederbach, Germany). Serum t-ucMGP levels were measured with a competitive ELISA, using a monoclonal antibody directed against the uncarboxylated MGP sequence 35-49, as described previously [17]. The intra-assay and inter-assay coefficients of variation were $8.9 \%$ and $11.4 \%$, respectively.

\section{Statistical analysis}

Continuous variables are presented as mean \pm standard deviation (SD) or as median with interquartile range (IQR). Patients were divided into tertiles according to t-ucMGP concentrations: low ( $\leq 129 \mathrm{nmol} / \mathrm{I})$, medium (130-188 nmol/l), and high (>189 nmol/l). Differences in clinical and biochemical characteristics between the patients in the t-ucMGP tertiles were analyzed by one-way ANOVA or by Kruskal-Wallis tests (for normally and non-normally distributed continuous variables, respectively) and $\chi^{2}$ tests (for categorical variables). Differences between HD and PD patients were analyzed by independent T-tests and Mann Whitney tests. Bivariate correlation analysis was performed to study the associations between t-ucMGP and clinical and biochemical variables. Multivariate regression analysis was used to adjust for potential confounders, which were age, rGFR, $\mathrm{K}_{\mathrm{t}} / \mathrm{V}$, hsCRP, calcium, and phosphorus levels. 
Cumulative mortality curves were calculated using Kaplan-Meier analysis for all-cause mortality. This method is known to profoundly overestimate the cumulative mortality when analyzing competing endpoints [25]. Separate analysis of CV mortality and non-CV mortality is a clear example of competing endpoints. For that reason, we calculated the cumulative mortality curves for $\mathrm{CV}$ mortality and non-CV mortality using competing risk analysis, taking account that patients dying for CV causes are no longer at risk to die for non-CV causes, and vice versa [26]. The survival time of patients was censored the time of transplantation, withdrawal from the study, or at the end of the study period (May 2009) with a maximum follow-up of five years. We calculated hazard ratios (HRs) for the tucMGP tertiles by Cox proportional hazard analysis, using the high t-ucMGP tertile as reference category. The multivariate models contained the variables age, sex, primary kidney disease, CVD, diabetes mellitus (DM), smoking, calcium, phosphorus, CRP, rGFR, nutritional status (SGA), and BMI.

A $p$ value of $<0.05$ was considered to be statistically significant; reported $p$ values are based on two-tailed tests of statistical significance. All statistical analyses were conducted using SPSS version 12.0 for Windows (SPSS Corp, Chicago, IL, USA). 


\section{Circulating total-uncarboxylated Matrix \\ Gla-protein (t-ucMGP) is not associated with \\ mortality in dialysis patients}

\section{Results}

Serum samples for t-ucMGP measurements were available for 952 patients from a total of 996 patients included in the NECOSAD cohort. Three patients were excluded from the analysis because of missing data regarding dialysis modality and survival. The mean t-ucMGP level of the total patient population ( $n=949$ ) was $165 \pm 67 \mathrm{nmol} / \mathrm{L}$, which is far below the normal range in healthy subjects $(300-700 \mathrm{nmol} / \mathrm{L}$; Figure 1$)$. The baseline characteristics of the patient population, 3 months after the start of dialysis treatment, are presented in Table 1 according to t-ucMGP tertiles (low, medium, high). Patients in the low t-ucMGP tertile were significantly older, had a higher prevalence of renal vascular disease as the primary cause of renal disease, moderate co-morbidities, and malnourishment (Table 1). There was no difference in the prevalence of CVD and DM among the t-ucMGP tertiles. The use of vitamin Kantagonists (coumarin derivatives) did not differ between the tertiles.

Figure 1: t-ucMGP values for hemodialysis (HD; $n=641$ ), peritoneal dialysis (PD; $n=308$ ) patients, and a healthy reference population (RP; $n=165)$

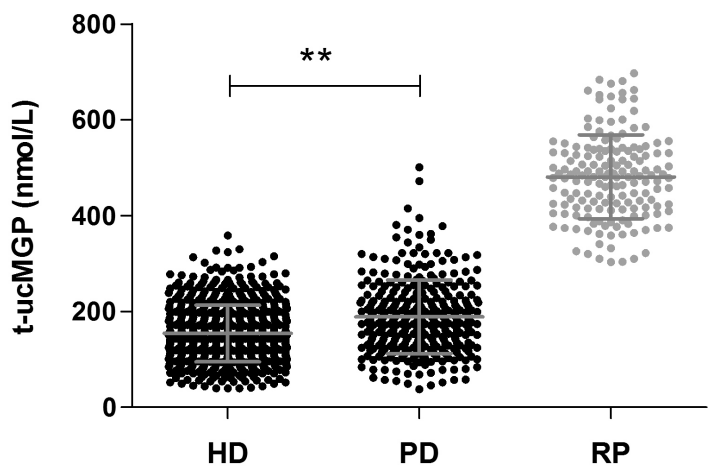

Circulating t-ucMGP levels in HD and PD patients and a previously described reference population [17] consisting of apparently healthy subjects aged between 20 and 80 years. Ninety-six percent of the dialysis patients had tucMGP levels below the normal range $(300-700 \mathrm{nmol} / \mathrm{L})$. PD patients had significantly higher t-ucMGP levels compared to hemodialysis patients $(* * p<0.001)$. 
Table 1: Baseline characteristics of the study population stratified according to t-ucMGP tertiles

\begin{tabular}{|c|c|c|c|c|}
\hline $\begin{array}{l}\text { Tertile } \\
\text { t-ucMGP (nmol/L) }\end{array}$ & $\begin{array}{l}\text { High } \\
\geq 189\end{array}$ & $\begin{array}{l}\text { Medium } \\
130-188\end{array}$ & $\begin{array}{l}\text { Low } \\
\leq 129\end{array}$ & P-value \\
\hline $\mathrm{N}$ & 315 & 309 & 325 & \\
\hline \multicolumn{5}{|l|}{ Demographic and clinical characteristics } \\
\hline Age (years) & $57 \pm 16^{\mathrm{a}}$ & $59 \pm 14^{a}$ & $62 \pm 15^{b}$ & $<0.001$ \\
\hline Sex (\% men) & 63 & 60 & 56 & 0.193 \\
\hline Dialysis modality (\% HD) & 55 & 70 & 77 & $<0.001$ \\
\hline Primary kidney disease (\%) & & & & $<0.001$ \\
\hline Diabetes & 16 & 15 & 15 & \\
\hline Glomerulonephritis & 15 & 16 & 8 & \\
\hline Renal vascular disease & 13 & 16 & 28 & \\
\hline Other & 57 & 54 & 50 & \\
\hline Co-morbidity (\%) & & & & 0.025 \\
\hline Low & 44 & 36 & 32 & \\
\hline Moderate & 32 & 36 & 42 & \\
\hline High & 24 & 28 & 26 & \\
\hline Diabetes (\%) & 22 & 24 & 22 & 0.855 \\
\hline Cardiovascular disease (\%) & 36 & 35 & 37 & 0.882 \\
\hline Current smoking (\%) & 25 & 26 & 19 & 0.172 \\
\hline \multicolumn{5}{|l|}{ Biochemical characteristics } \\
\hline $\mathrm{rGFR}(\mathrm{ml} / \mathrm{min})^{*}$ & $4.1(2.0-6.4)^{\mathrm{a}}$ & $2.9(1.1-4.9)^{b}$ & $3.2(1.6-5.0)^{b}$ & $<0.001$ \\
\hline Nutritional status (\% malnourished) & 21 & 29 & 33 & 0.002 \\
\hline $\mathrm{BMI}\left(\mathrm{kg} / \mathrm{m}^{2}\right)$ & $25 \pm 4$ & $25 \pm 5$ & $24 \pm 4$ & 0.081 \\
\hline $\mathrm{hsCRP}(\mathrm{mg} / \mathrm{L})^{*}$ & $4.3(1.7-10.2)$ & $4.2(1.7-13.3)$ & $5.5(1.7-15.5)$ & 0.198 \\
\hline Albumin (g/L) & $36 \pm 4.8$ & $36 \pm 5.5$ & $36 \pm 5.2$ & 0.447 \\
\hline Hemoglobin (nmol/L) & $7.1 \pm 0.9^{a}$ & $6.8 \pm 1.0^{b}$ & $6.8 \pm 1.1^{b}$ & $<0.001$ \\
\hline Corrected calcium (mmol/L) & $2.5 \pm 0.2^{a}$ & $2.4 \pm 0.3^{\mathrm{ab}}$ & $2.4 \pm 0.3^{b c}$ & 0.003 \\
\hline Phosphorus (mmol/L) & $1.8 \pm 0.5^{\mathrm{a}}$ & $1.9 \pm 0.5^{\mathrm{bc}}$ & $1.9 \pm 0.6^{\mathrm{ab}}$ & 0.039 \\
\hline iPTH (pmol/L)* & $11.9(4.2-28.0)$ & $12.9(5.5-29.3)$ & $12.3(4.2-29.0)$ & 0.625 \\
\hline Pulse pressure (mmHg) & $61 \pm 17^{\mathrm{a}}$ & $63 \pm 17^{\mathrm{a}}$ & $66 \pm 16^{b}$ & $<0.001$ \\
\hline Use of coumarin derivatives (\%) & 12 & 14 & 14 & 0.762 \\
\hline
\end{tabular}

Demographic, clinical and biochemical characteristics of patients stratified according to t-ucMGP tertiles: high ( $\geq 189 \mathrm{nmol} / \mathrm{L})$, medium (130-188 nmol/L), and low ( $\leq 129 \mathrm{nmol} / \mathrm{L})$. Data are presented as number and percentage of the t-ucMGP tertile, mean \pm standard deviation, or as median and IQR range (indicated with *). Abbreviations used: rGFR, residual glomerular filtration rate; BMI, body mass index; hsCRP, high-sensitivity C-reactive protein; iPTH, intact parathyroid hormone.

\section{t-ucMGP and dialysis modality}

The percentage of patients who underwent HD treatment was highest in the low t-ucMGP tertile (Table 1). Patients undergoing PD treatment had significantly higher t-ucMGP levels $(189 \pm 77 \mathrm{nmol} / \mathrm{L})$ compared to HD patients (154 $\pm 59 \mathrm{nmol} / \mathrm{L}, \mathrm{p}<0.001$; Figure 1$)$. The difference in t-ucMGP levels persisted after correction for age, sex, primary kidney disease, CVD, DM, rGFR, hs-CRP, calcium, phosphorus, and use of coumarin derivatives in multivariate linear regression analysis $(p=0.001)$. 


\section{Circulating total-uncarboxylated Matrix Gla-protein (t-ucMGP) is not associated with mortality in dialysis patients}

t-ucMGP correlations with other relevant parameters

Circulating t-ucMGP levels significantly correlated with $r G F R(r=0.136, p<0.001), K_{t} / V(r=-$ 0.073, $p=0.029)$, hsCRP ( $r=-0.068, p=0.035)$, calcium ( $r=0.105, p=0.001)$, and phosphorus levels $(r=-0.700, p=0.032)$. There was no significant correlation between $t$-ucMGP and iPTH $(p=0.683)$. Circulating t-ucMGP levels negatively correlated with pulse pressure $(r=-$ $0.157, p<0.001$ ), which persisted after correction for age, $r G F R, K_{t} / V, h s C R P$, calcium, and phosphorus levels in multivariate regression analysis ( $B$ for $t-u c M G P=-0.088 ; p=0.010$ ).

\section{t-ucMGP and survival}

Overall, five-year patient survival was $61.3 \%$. Median follow-up time was 862 days (IQR 410-1498). Censoring was observed in 488 patients. Of the total mortality (367 patients), approximately half (48.8\%) was attributed to CV death and half (51.2\%) to non-CV death. Common causes of non-CV death were infections $(n=41,11.2 \%)$, refusal of further treatment $(n=40,10.9 \%)$, cessation of therapy for any other reason $(n=31,8.5 \%)$, and malignant diseases $(n=29,7.9 \%)$. After 5 years, 278 HD patients $(43.4 \%)$ had died and 89 PD patients (28.9\%).

Figure 2 shows the crude survival curves for overall, CV and non-CV mortality of the total study population. Table 2 shows the adjusted hazard ratios (HR) for overall, CV and non$\mathrm{CV}$ death for the low and medium t-ucMGP tertiles compared with the high t-ucMGP tertile (reference group) for HD and PD patients. PD patients in the low and medium tucMGP tertiles had significantly increased risks of non-cardiovascular mortality of 2.12 (95\% Cl 1.01-4.44) and 2.32 (95\% Cl 1.10-4.91), respectively. The non-CV mortality risk remained high for PD patients in the medium and low ucMGP tertiles when adjusting for age, sex, primary kidney disease, CVD, DM, smoking, calcium, phosphorus, CRP, rGFR, malnourishment, and $\mathrm{BMI}(2.60$ [95\% Cl 1.02-6.16] and 2.50 [95\% Cl 1.02-6.16], respectively). For HD patients, neither the low nor the medium t-ucMGP tertile had an increased mortality risk in the unadjusted or adjusted Cox-regression analysis (Table 2). In the total study population, there were no significant differences between the t-ucMGP tertiles (data not shown). 
Figure 2: Mortality curves.

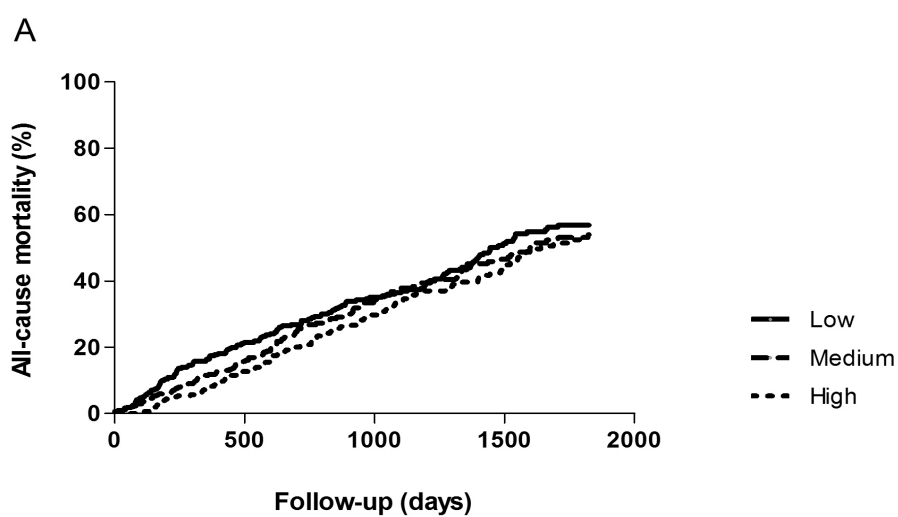

B

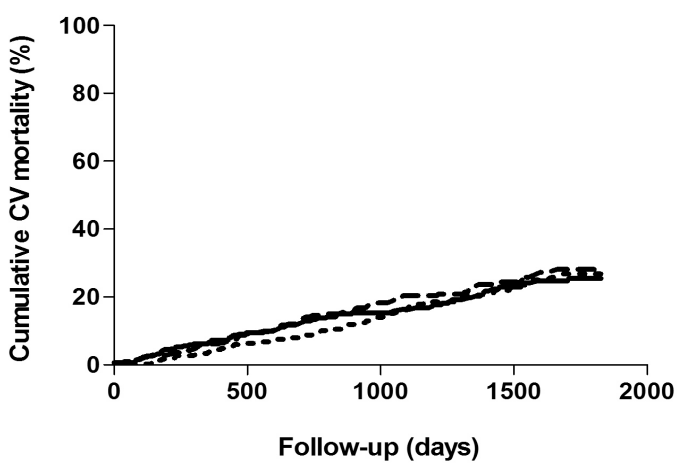

- Low

-- Medium

... High

C

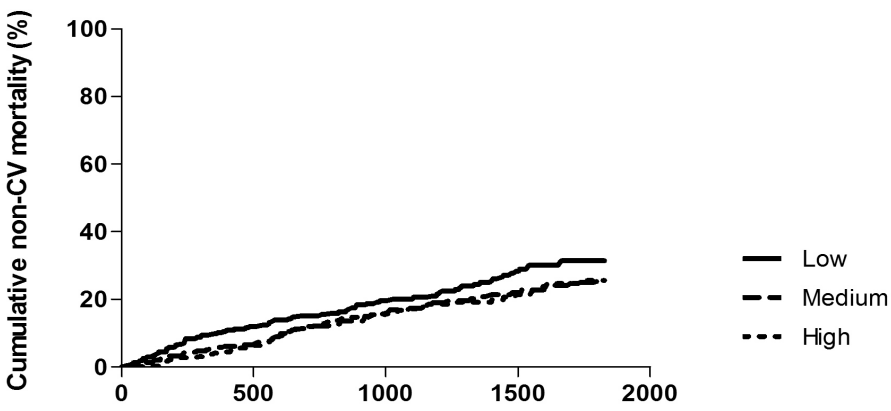

Follow-up (days)

Mortality curves showing all-cause (A), cardiovascular (B), and non-cardiovascular (C) mortality by tertile of tucMGP level (high $\geq 189 \mathrm{nmol} / \mathrm{L}$, medium 130-188 nmol/L, and low $\leq 129 \mathrm{nmol} / \mathrm{L}$ ) for the total study population. The latter two curves are based on competing-risks analysis. 


\section{Circulating total-uncarboxylated Matrix Gla-protein (t-ucMGP) is not associated with mortality in dialysis patients}

Table 2: Cox-regression analysis for the association of t-ucMGP tertiles with overall, CV and non-CV mortality in hemodialysis (A) and peritoneal dialysis (B) patients

\begin{tabular}{|c|c|c|c|c|c|c|}
\hline \multirow{3}{*}{$\begin{array}{l}\text { A. HD patients } \\
\mathrm{N} \\
\text { t-ucMGP tertile }\end{array}$} & \multicolumn{2}{|c|}{ Overall mortality } & \multicolumn{2}{|c|}{ Cardiovascular mortality } & \multicolumn{2}{|c|}{ Non-cardiovascular mortality } \\
\hline & \multicolumn{2}{|c|}{278} & \multicolumn{2}{|c|}{135} & \multicolumn{2}{|c|}{143} \\
\hline & HR & $95 \% \mathrm{Cl}$ & HR & $95 \% \mathrm{Cl}$ & HR & $95 \% \mathrm{Cl}$ \\
\hline \multicolumn{7}{|l|}{ Model 1} \\
\hline Low & 1.06 & $0.80-1.42$ & 1.09 & $0.71-1.67$ & 1.03 & $0.70-1.53$ \\
\hline Medium & 0.89 & $0.65-1.21$ & 1.04 & $0.67-1.62$ & 0.76 & $0.49-1.17$ \\
\hline High & 1.0 & & 1.0 & & 1.0 & \\
\hline \multicolumn{7}{|l|}{ Model 2} \\
\hline Low & 1.05 & $0.78-1.41$ & 1.11 & $0.72-1.70$ & 1.00 & $0.67-1.50$ \\
\hline Medium & 0.87 & $0.64-1.19$ & 1.05 & $0.67-1.63$ & 0.72 & $0.46-1.12$ \\
\hline High & 1.0 & & 1.0 & & 1.0 & \\
\hline \multicolumn{7}{|l|}{ Model 3} \\
\hline Low & 0.96 & $0.68-1.36$ & 1.01 & $0.61-1.68$ & 0.93 & $0.57-1.53$ \\
\hline Medium & 0.79 & $0.54-1.15$ & 0.83 & $0.49-1.40$ & 0.76 & $0.45-1.31$ \\
\hline High & 1.0 & & 1.0 & & 1.0 & \\
\hline B. PD patients & \multicolumn{2}{|c|}{ Overall mortality } & \multicolumn{2}{|c|}{ Cardiovascular mortality } & \multicolumn{2}{|c|}{ Non-cardiovascular mortality } \\
\hline $\mathrm{N}$ & 89 & & 44 & & 45 & \\
\hline t-ucMGP tertile & HR & $95 \% \mathrm{Cl}$ & HR & $95 \% \mathrm{Cl}$ & HR & $95 \% \mathrm{Cl}$ \\
\hline \multicolumn{7}{|l|}{ Model 1} \\
\hline Low & 1.35 & $0.79-2.30$ & 0.74 & $0.32-1.68$ & 2.32 & $1.10-4.91$ \\
\hline Medium & 1.64 & $1.01-2.67$ & 1.34 & $0.69-2.57$ & 2.12 & $1.01-4.44$ \\
\hline High & 1.0 & & 1.0 & & 1.0 & \\
\hline \multicolumn{7}{|l|}{ Model 2} \\
\hline Low & 1.30 & $0.75-2.24$ & 0.68 & $0.29-1.59$ & 2.29 & \\
\hline Medium & 1.72 & $1.04-2.84$ & 1.52 & $0.77-2.99$ & 2.05 & \\
\hline High & 1.0 & & 1.0 & & 1.0 & \\
\hline \multicolumn{7}{|l|}{ Model 3} \\
\hline Low & 1.31 & $0.72-2.39$ & 0.75 & $0.30-1.87$ & 2.50 & $1.02-6.16$ \\
\hline Medium & 1.30 & $0.75-2.26$ & 0.82 & $0.38-1.79$ & 2.60 & $1.09-6.21$ \\
\hline High & 1.0 & & 1.0 & & 1.0 & \\
\hline
\end{tabular}

Model 1, crude.

Model 2, age, sex, primary kidney disease, CVD, DM, smoking

Model 3, age, sex, primary kidney disease, CVD, DM, smoking, calcium, phosphorus, CRP, rGFR, malnourishment, BMI

Results of Cox-regression analysis showing the hazard ratio of the low and medium t-ucMGP tertile compared with the high t-ucMGP tertile for overall, $\mathrm{CV}$ and non-CV mortality for hemodialysis $(\mathrm{A})$ and peritoneal dialysis (B) patients. Abbreviations used: $\mathrm{CV}$, cardiovascular; $\mathrm{HR}$, hazard ratio; $\mathrm{Cl}$, confidence interval. 


\section{Discussion}

We determined serum t-ucMGP levels in a prospective, well-defined Dutch cohort of 949 incident dialysis patients to investigate the potential predictive value of circulating t-ucMGP for overall and cardiovascular mortality. Almost all patients (>96\%) undergoing dialysis treatment for 3 months had circulating t-ucMGP levels far below the normal range. PD patients had significantly higher t-ucMGP levels compared to HD patients, but these levels were still very low when compared to healthy subjects (normal range 300-700 nmol/L). When patients were divided into tertiles according to t-ucMGP levels, we found no difference between high, medium, and low circulating t-ucMGP levels for mortality risk.

The finding of low t-ucMGP levels in dialysis patients is in accordance with previous reports [17-19]. However, this is the first study that demonstrates in a large cohort that patients who started recently with dialysis treatment ( 3 months) already have low $\mathrm{t}$ ucMGP levels. This may be explained by the fact that all patients have reached the final stadium of chronic kidney disease. It has been shown that circulating t-ucMGP levels are inversely correlated with GFR in patients with stable CVD [27]. Moreover, in patients with aortic valve disease, those with CKD stage 4 had significantly lower t-ucMGP levels compared to patients with normal to moderately impaired renal function (CKD stage 1-3) [28]. It is therefore likely that circulating t-ucMGP levels are already lowered in the previous stages of CKD, and when patients have developed ESRD, apparently the majority of patients has very low t-ucMGP levels.

Since virtually all patients of the NECOSAD cohort had very low t-ucMGP levels, the tucMGP tertiles all represent relatively low t-ucMGP levels. This may contribute to the absence of a difference in mortality risk between the tertiles: whether a patient has a low or very low t-ucMGP level does not influence his or her mortality risk. However, the current finding of very low t-ucMGP levels in virtually all patients of the NECOSAD cohort supports the hypothesis that dialysis patients have a changed MGP status, which may contribute to the development of cardiovascular calcification in this patient population.

The present study is the first to investigate circulating t-ucMGP levels in a large group of PD patients and it is obvious that also patients undergoing this particular type of renal replacement therapy had low t-ucMGP levels. On average, HD patients had even lower tucMGP levels, but the difference was relatively small. It has been found in the NECOSAD cohort that patients with moderate to severe aortic arch calcifications were more often treated with HD than PD[29]. From the patients without calcification, the majority (55\%) underwent PD treatment. 


\section{Circulating total-uncarboxylated Matrix Gla-protein (t-ucMGP) is not associated with mortality in dialysis patients}

The higher t-ucMGP levels in PD patients might therefore be explained by less (severe) arterial calcification found in these patients. Residual confounding may be another explanation of the found difference in t-ucMGP levels between PD and HD patients.

Patients in the low t-ucMGP tertile were significantly older, had more renal vascular disease, moderate co-morbidities, and malnourishment. There was no difference in the prevalence of CVD and DM between the t-ucMGP tertiles. It has been previously found, using the same MGP assay, that patients with CVD have significantly lower t-ucMGP levels compared to healthy subjects $[17,27,28]$. However, the t-ucMGP levels of these patients were higher compared to t-ucMGP levels of dialysis patients [17]. The presence of ESRD seemed to result in a more extensive lowering of t-ucMGP levels than the presence of CVD. In the present cohort, in which virtually all patients have very low t-ucMGP levels, the effect of CVD on tucMGP levels in the presence of ESRD might not be detected. Circulating t-ucMGP correlated inversely with pulse pressure in the total patient population; this association persisted after correction for age, $\mathrm{rGFR}, \mathrm{K}_{\mathrm{t}} / \mathrm{V}$, hsCRP, calcium, and phosphate levels. In agreement, $\mathrm{t}$ ucMGP has been reported to correlate inversely with the aortic augmentation index and the coronary artery calcification score in dialysis patients $[18,20]$. Altogether, this suggests that t-ucMGP may serve as a biomarker for prevalent arterial calcification.

In the PD population, there was a significant difference between the t-ucMGP tertiles for non-CV death, which remained statistically significant when adjusting for several clinical and biochemical variables in Cox regression analysis. It should be noted, however, that the $95 \%$ confidence intervals were broad and that only 45 deaths accounted for this observation. In addition, for some patients the cause of death was uncertain or could not be determined. These deaths were classified as cardiovascular deaths, since the majority of these patients died acutely, pointing to a cardiovascular cause. This might result in an overestimation of the number of CV deaths, and analyses were therefore repeated with these deaths classified as non-CV. The differences between the t-ucMGP tertiles in Cox-regression analysis for non$\mathrm{CV}$ death among PD patients were then no longer significant (data not shown).

One of the limitations of the present study was that the mortality data relied on observations of treating physicians. This could induce some error in the definition of the exact cause of death. The classification of deaths with an uncertain or missing cause as CV mortality did influence our results, as previously described for PD patients. A major limitation was that no sensitive imaging technique such as multislice computed tomography scanning was applied to evaluate the precise amount of arterial calcification in the current population. 
The relationship between t-ucMGP, the extent of vascular calcifications, and mortality could therefore not be evaluated in the present study.

In conclusion, this is the first study in which circulating t-ucMGP was determined in a large number of incident HD as well as PD patients. Serum t-ucMGP levels were extremely low in almost all patients, which may explain the fact that mortality risk did not differ between the t-ucMGP tertiles. Low circulating t-ucMGP levels indicate a changed MGP status, which may contribute to the high vascular calcification-risk in dialysis patients. However, further investigation is needed to confirm this. 


\section{Circulating total-uncarboxylated Matrix \\ Gla-protein (t-ucMGP) is not associated with \\ mortality in dialysis patients}

\section{Acknowledgments}

We thank The Netherlands Cooperative Study on the Adequacy of Dialysis trial nurses and data managers for data collection and management. We thank M-L. Boumans for her excellent work in MGP analysis.

NECOSAD study group members: Apperloo AJ, Bijlsma JA, Boekhout $M$, Boer WH, Boog PJM van der, Büller HR, Buren M van, Charro FTh de, Doorenbos CJ, Dorpel MA van den, Es A van, Fagel WJ, Feith GW, Fijter CWH de, Frenken LAM, Grave W, Geelen JACA van, Gerlag PGG, Gorgels JPMC, Huisman RM, Jager KJ, Jie K, Koning-Mulder WAH, Koolen MI, Kremer Hovinga TK, Lavrijssen ATJ, Luik AJ, Parlevliet KJ, Raasveld MHM, Sande FM van der, Schonck MJM, Schuurmans MMJ, Siegert CEH, Stegeman CA, Stevens P, Thijssen JGP, Valentijn RM, Vastenburg GH, Verburgh CA, Verstappen VMC, Vincent HH, Vos PF.

\section{References}

1. U.S. Renal Data System, USRDS 2009 Annual Data Report: Atlas of End-Stage Renal Disease in the United States, National Institutes of Health, National Institute of Diabetes and Digestive and Kidney Diseases, Bethesda, MD, 2009.

2. Blacher, J., et al., Arterial calcifications, arterial stiffness, and cardiovascular risk in end-stage renal disease. Hypertension, 2001. 38(4): p. 938-42.

3. London, G.M., et al., Arterial media calcification in end-stage renal disease: impact on all-cause and cardiovascular mortality. Nephrol Dial Transplant, 2003. 18(9): p. 1731-40.

4. London, G.M., et al., Arterial structure and function in end-stage renal disease. Nephrol Dial Transplant, 2002. 17(10): p. 1713-24.

5. Price, P.A., M.R. Urist, and Y. Otawara, Matrix Gla protein, a new gamma-carboxyglutamic acid-containing protein which is associated with the organic matrix of bone. Biochem Biophys Res Commun, 1983. 117(3): p. 765-71.

6. Price, P.A. and M.K. Williamson, Primary structure of bovine matrix Gla protein, a new vitamin K-dependent bone protein. J Biol Chem, 1985. 260(28): p. 14971-5.

7. Price, P.A., J.S. Rice, and M.K. Williamson, Conserved phosphorylation of serines in the Ser-X-Glu/Ser(P) sequences of the vitamin K-dependent matrix Gla protein from shark, lamb, rat, cow, and human. Protein Sci, 1994. 3(5): p. 822-30.

8. Schurgers, L.J., E.C.M. Cranenburg, and C. Vermeer, Matrix Gla-protein: The calcification inhibitor in need of vitamin K. Thromb Haemost, 2008. 100(4): p. 593-603.

9. Wallin, R., et al., Modulation of the binding of matrix Gla protein (MGP) to bone morphogenetic protein-2 (BMP-2). Thromb Haemost, 2000. 84(6): p. 1039-44.

10. Wallin, R., L.J. Schurgers, and N. Wajih, Effects of the blood coagulation vitamin $K$ as an inhibitor of arterial calcification Thromb Res 2008. 122(3): p. 411-7.

11. Bostrom, K., et al., Matrix GLA protein modulates differentiation induced by bone morphogenetic protein-2 in C3H10T1/2 cells. J Biol Chem, 2001. 276(17): p. 14044-52.

12. Price, P.A., S.A. Faus, and M.K. Williamson, Warfarin causes rapid calcification of the elastic lamellae in rat arteries and heart valves. Arterioscler Thromb Vasc Biol, 1998. 18(9): p. 1400-7. 
13. Price, P.A., A.M. Roublick, and M.K. Williamson, Artery calcification in uremic rats is increased by a low protein diet and prevented by treatment with ibandronate. Kidney Int, 2006. 70(9): p. 1577-83.

14. Price, P.A., S.A. Faus, and M.K. Williamson, Warfarin-induced artery calcification is accelerated by growth and vitamin D. Arterioscler Thromb Vasc Biol, 2000. 20(2): p. 317-27.

15. Sweatt, A., et al., Matrix Gla protein (MGP) and bone morphogenetic protein-2 in aortic calcified lesions of aging rats. J Thromb Haemost, 2003. 1(1): p. 178-85.

16. Schurgers, L.J., et al., Novel conformation-specific antibodies against matrix gamma-carboxyglutamic acid (Gla) protein: undercarboxylated matrix Gla protein as marker for vascular calcification. Arterioscler Thromb Vasc Biol, 2005. 25(8): p. 1629-33.

17. Cranenburg, E.C., et al., The circulating inactive form of Matrix Gla Protein (ucMGP) as a biomarker for cardiovascular calcification J Vasc Res 2008. 45: p. 427-36.

18. Hermans, M.M., et al., Undercarboxylated Matrix GLA Protein Levels Are Decreased in Dialysis Patients and Related to Parameters of Calcium-Phosphate Metabolism and Aortic Augmentation Index. Blood Purif, 2007. 25(5): p. 395-401.

19. Shroff, R.C., et al., The circulating calcification inhibitors, fetuin-A and osteoprotegerin, but not Matrix Gla protein, are associated with vascular stiffness and calcification in children on dialysis. Nephrol Dial Transplant, 2008. 23(10): p. 3263-71.

20. Cranenburg, E.C.M., et al., Uncarboxylated matrix Gla protein (UCMGP) is associated with coronary artery calcification in haemodialysis patients. Thromb Haemost, 2009. 101(2): p. 359-66.

21. van Dijk, P.C., et al., Renal replacement therapy in Europe: the results of a collaborative effort by the ERAEDTA registry and six national or regional registries. Nephrol Dial Transplant, 2001. 16(6): p. 1120-9.

22. Khan, I.I.H., et al., Influence of coexisting disease on survival on renal-replacement therapy. Lancet, 1993. 341(8842): p. 415-8.

23. Watson, P.E., I.D. Watson, and R.D. Batt, Total body water volumes for adult males and females estimated from simple anthropometric measurements. Am J Clin Nutr, 1980. 33(1): p. 27-39.

24. Daugirdas, J.T., Second generation logarithmic estimates of single-pool variable volume Kt/V: an analysis of error. J Am Soc Nephrol, 1993. 4(5): p. 1205-13.

25. Southern, D.A., et al., Kaplan-Meier methods yielded misleading results in competing risk scenarios. J Clin Epidemiol, 2006. 59(10): p. 1110-4.

26. Satagopan, J.M., et al., A note on competing risks in survival data analysis. Br J Cancer, 2004. 91(7): p. 122935.

27. Parker, B.D., et al., Association of kidney function and uncarboxylated matrix Gla protein: Data from the Heart and Soul Study. Nephrol Dial Transplant, 2009. 24(7): p. 2095-101.

28. Koos, R., et al., Relation of circulating Matrix Gla-Protein and anticoagulation status in patients with aortic valve calcification. Thromb Haemost, 2009. 101(4): p. 706-13.

29. Noordzij, M., et al., Progression of aortic calcification is associated with disorders of mineral metabolism and mortality in chronic dialysis patients. Nephrol Dial Transplant, 2010. 


\section{CHAPTER 5}

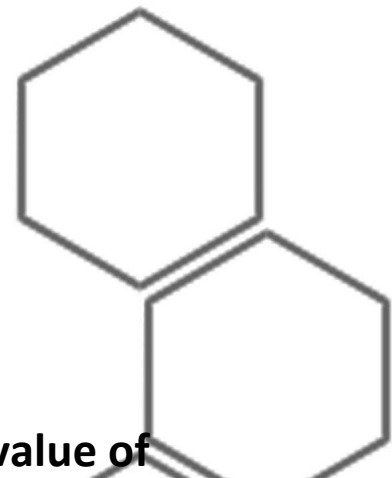

circulating Matrix Gla-protein (MGP) species

Ellen C.M. Cranenburg ${ }^{1}$, Ralf Koos ${ }^{2}$, Leon J. Schurgers ${ }^{1}$, Elke J. Magdeleyns ${ }^{1}$, Thea H.M. Schoonbrood ${ }^{3}$, Robert B. Landewé ${ }^{3}$, Vincent M. Brandenburg ${ }^{2 / 4}$, Otto Bekers ${ }^{5}$, Cees Vermeer $^{1}$

${ }^{1}$ VitaK and Cardiovascular Research Institute Maastricht (CARIM), Maastricht University, Maastricht, The Netherlands; ${ }^{2}$ Department of Cardiology, RWTH University Hospital Aachen, Aachen, Germany; ${ }^{3}$ Department of Rheumatology, University Hospital Maastricht, Maastricht, the Netherlands; ${ }^{4}$ Department of Nephrology and Clinical Immunology, RWTH University Hospital Aachen, Aachen, Germany; ${ }^{5}$ Department of Clinical Chemistry, University Hospital Maastricht, Maastricht, the Netherlands 


\begin{abstract}
Matrix $\gamma$-carboxyglutamate (Gla)-protein (MGP) is an important local inhibitor of vascular calcification, which can undergo two post-translational modifications: vitamin Kdependent $\gamma$-glutamate carboxylation and serine phosphorylation. While carboxylation is thought to have effects upon binding of calciumions, phosphorylation is supposed to affect the cellular release of MGP. Since both modifications can be exerted incompletely, various MGP species can be detected in the circulation.

MGP levels were measured with two commercially available competitive and two novel sandwich assays in healthy controls, in patients with rheumatic disease (RD), aortic valve disease (AVD), and end-stage renal disease (ESRD), as well as in volunteers after vitamin $\mathrm{K}$ supplementation (VKS) and treatment with vitamin K antagonists (VKA). Major differences were found between the MGP assays, including significantly different behaviour with regard to vascular disease and the response to VKA and VKS. The sandwich assay measuring non-phosphorylated, non-carboxylated MGP (dp-ucMGP) was particularly sensitive for these changes and would be suited to assess the vascular vitamin $\mathrm{K}$ status.
\end{abstract}

We conclude that the different assays for particular circulating MGP species allows the assessment of various aspects of the MGP system. 


\section{Characterisation and potential diagnostic value of circulating matrix Gla-protein (MGP) species}

\section{Introduction}

Matrix $\gamma$-carboxyglutamate (Gla)-protein (MGP) has gained increasing attention as an important local inhibitor of vascular calcification [1]. MGP is a small secretory protein synthesized by vascular smooth muscle cells (VSMC) and chondrocytes [2-4]. It may undergo two post-translational modifications: vitamin K-dependent $\mathrm{\gamma}$-glutamate carboxylation and serine phosphorylation [5-7]. MGP is thought to exert its function as a calcification inhibitor by binding to crystal nuclei in hydroxyapaptite, thereby preventing crystal growth $[8,9]$. Additionally, MGP is able to bind and inhibit bone morphogenetic protein-2, an osteogenic growth factor [10-12]. For both mechanisms of action, MGP has to be carboxylated $[10,13]$. The exact function of the phosphoserine residues is unclear, but recent findings indicate that they may regulate the secretion of MGP into the extracellular environment [14]. These negatively-charged residues may also contribute to the binding of MGP to calcium, since MGP retained its affinity for hydroxyapatite after thermal decarboxylation and non-carboxylated MGP accumulated at sites of calcification [8,15-17]. It has been shown in MGP-deficient mice that circulating MGP has little or none calcification-inhibitory activity [18].

MGP may exist as various species according to their state of phosphorylation and/or carboxylation (phosphorylated [pMGP], non-phosphorylated [desphospho, dpMGP], carboxylated [cMGP] or uncarboxylated [UCMGP]). Hence, in the circulation different MGP species may be detected including p-cMGP, p-ucMGP, dp-cMGP, and dp-ucMGP. In the vascular tissue, the affinity of these species for calcium salts and their calcification-inhibitory activity may differ widely. These various circulating MGP species may reflect the degree of calcification (inhibition) in the vascular wall and, since MGP carboxylation is vitamin Kdependent, certain species may also reflect the availability of vitamin $\mathrm{K}$ present in the vascular wall. Clinically, an insufficient vascular vitamin $\mathrm{K}$ status may result in an increased risk for arterial calcification [19-21]. Vitamin $\mathrm{K}$ occurs as vitamin $\mathrm{K}_{1}$ (phylloquinone) and vitamin $\mathrm{K}_{2}$ (menaquinones). Menaquinones are classified according to their aliphatic side chain length (MK-n) [22]. It has been demonstrated that MK-7 has a half-life time of several days and possess a higher efficacy in extra-hepatic Gla-protein carboxylation [23].

Unfortunately, there are currently no assays available to measure each circulating MGP species or even the total circulating MGP pool. In the literature, three different assays measuring circulating MGP have been described so far: one radio-immunoassay [24] and two competitive (mono-antibody) enzyme-linked immunosorbent assays (ELISA) [25, 26]. The first assay is not commercially available and was therefore not used in the current study. 
The latter two MGP ELISA quantify total dpMGP (t-dpMGP; Biomedica, Vienna, Austria) and total ucMGP (t-ucMGP; VitaK, Maastricht, the Netherlands). With the t-ucMGP assay, uniformly low levels have been measured in cardiovascular disease (CVD) and chronic kidney disease patients [26-29]. In contrast, with the t-dpMGP assay, both low and high MGP levels have been measured in patients with CVD [17, 25, 30]. A direct comparison between these two assays has not yet been performed.

We aimed to develop new, sandwich (dual-antibody) MGP assays which could detect both uncarboxylated and carboxylated MGP species. We have developed two assays detecting circulating dp-ucMGP and dp-cMGP. Due to the absence of phosphoserine residues, we hypothesized that these MGP species may be more easily set free into the circulation, independent of the presence of calcification in the vascular wall, and may therefore mainly be influenced by the availability of vitamin K. Changes in systemic vitamin K status, for instance resulting from the use of vitamin $\mathrm{K}$ supplementation (VKS) or vitamin $\mathrm{K}$ antagonists (VKA, coumarin derivatives), should then be reflected in circulating MGP measurements. This was tested in the current study.

In recent years, biomarkers assessing either the risk to develop vascular calcification or the extent of prevalent calcification have become of substantial importance. The prognostic value of cardiovascular calcification has already been clearly established in patients with cardiovascular disease, including aortic valve disease (AVD), and in patients with endstage renal disease (ESRD) [31-33]. Moreover, increasingly more high-risk patient populations are being identified, including patients with systemic lupus erythematosus (SLE) and rheumatoid arthritis (RA) [34-36]. In the present study, MGP levels were measured in these patient populations in order to compare the two available and the two newlydeveloped MGP assays and to evaluate their potential utility for disease monitoring and risk assessment. In addition, patients with cartilage calcification/destruction (chondrocalcinosis [CC] and gout) and soft tissue calcification (diffuse idiopathic skeletal hyperostosis [DISH], also known as Forestier's disease) were included to determine whether nonvascular pathologies would influence MGP levels. Finally, to test the suitability of the new $\mathrm{dp}$-ucMGP and dp-cMGP assays for assessing vascular vitamin $\mathrm{K}$ status, MGP levels were measured in healthy subjects receiving VKS and VKA. 


\section{Characterisation and potential diagnostic value of circulating matrix Gla-protein (MGP) species}

\section{Materials and methods}

\section{$\underline{\text { Subjects }}$}

Seventy-five apparently healthy subjects, aged between 25 and 80 years (y), were recruited from the general Maastricht population to serve as reference population (RP) and to determine the characteristics of the dp-ucMGP and dp-cMGP assays. These subjects had no overt history of cardiovascular, chronic kidney, and/or rheumatic diseases and did not use VKS or VKA. Patient samples were acquired from a total of 146 patients diagnosed with either rheumatic disease (RD $n=51), \operatorname{AVD}(n=50)$, or ESRD $(n=45)$ patients.

The RD population was diagnosed with either CC $(n=9)$, gout $(n=13), \operatorname{DISH}(n=4), \operatorname{RA}$ $(n=16)$, and SLE $(n=9)$ from the division Rheumatology of the University Hospital Maastricht (Maastricht, the Netherlands). RD patients with a history of CVD and/or CKD were excluded. Data were obtained on demographic and clinical characteristics during patient interviews as well as review of patients' files.

Patients with calcific AVD were recruited at the Echocardiography or Cardiology department of the Rheinisch-Westfälische Technische Hochschule (RWTH) University Hospital Aachen, Germany, as described previously [28].

The ESRD patient population consisted of 45 prevalent hemodialysis (HD) patients from three dialysis centers cooperating with the RWTH University Hospital Aachen, as described previously [27]. Etiologies for ESRD were glomerulonephritis/vasculitis ( $n=16)$, tubulointerstitial disease $(n=3)$, amyloid nephropathy $(n=2)$, vascular disease/hypertension $(n=4)$, hereditary disease/cystic disease $(n=6)$, diabetic kidney disease $(n=4)$, and miscellaneous $(n=10)$. From all patient populations, patients using VKA were excluded.

To determine the effect of changes in systemic vitamin $\mathrm{K}$ status on MGP levels, 2 groups of healthy volunteers were included: 52 subjects receiving VKS and 17 subjects receiving VKA. The VKS group consisted of 24 men and women aged between 20 and $40 \mathrm{y}$ (mean \pm SD age $28 \pm 7$ y) receiving $45,90,180$ or $360 \mu$ g menaquinone-7 (MenaQ7, NattoPharma, Oslo, Norway) daily during 12 weeks and of 28 postmenopausal women aged between 55 and $75 \mathrm{~s}(70 \pm 11 \mathrm{y})$ receiving $45 \mathrm{mg}$ menaquinone-4 (menatetrenone, EISAI Co, Tokyo, Japan) daily for 6 months. The subjects who received MK-7 as well as those receiving VKA were part of a separate study investigating supplementation with vitamin $\mathrm{K} 2$ and oral anticoagulant treatment (E. Theuwissen et al., unpublished data). The postmenopausal women of the MK-4 group were part of a clinical trial investigating the effect of MK-4 on bone mineral density and bone strength [37]. For comparison, 11 women $(76 \pm 14 \mathrm{y})$ from the same clinical trial receiving placebo were included. 
The VKA group consisted of 17 subjects, aged between 20 and $45 \mathrm{y}$. These subjects received acenocoumarol (Sintrom mitis $1 \mathrm{mg}$, Novartis Pharma, Arnhem, the Netherlands) in a once-daily dose (mean \pm SD daily dose $2.8 \pm 1.0 \mathrm{mg} \mathrm{mg}$ ) to achieve a target international normalized ratio (INR) of 2.0. Blood samples obtained at baseline and after 4 weeks, when a stable INR of 2.0 was reached, were used for MGP measurements. Subjects with a history of coagulation disorders, metabolic, gastrointestinal, and/or chronic inflammatory diseases and subjects using corticosteroids, VKS, or VKA were excluded.

The study was approved by the local Medical Ethics Committees and informed consent was obtained from all participants.

\section{Imaging procedure}

In 33 AVD patients, echocardiography was performed as described previously [28] and according to the ACC/AHA guidelines [38]. Patients with a transaortic flow velocity of 2.5$3.0 \mathrm{~m} / \mathrm{s}$ were classified as mild aortic stenosis, those with a velocity of $3.0-4.0 \mathrm{~m} / \mathrm{s}$ as moderate, and those with a velocity of $>4.0 \mathrm{~m} / \mathrm{s}$ as severe aortic stenosis [38]. Due to the low number of patients with severe aortic stenosis $(n=3)$, the latter 2 groups were considered as one category for analysis (moderate to severe aortic stenosis).

\section{Biochemical measurements}

Citrated plasma and serum from all participants was prepared by standard centrifugation and stored at $-80{ }^{\circ} \mathrm{C}$ until testing. From 4 healthy subjects (age $26 \pm 6 \mathrm{y}$ ), also EDTA and heparin plasma was prepared to determine the matrix effect on dp-ucMGP and dp-cMGP levels. Venous blood samples from the ESRD patients were taken at the start of HD after a long dialysis interval. Circulating MGP levels were measured with 4 different MGP assays, which are described below and in Table 1. Calcium, phosphate, hemoglobin, alkaline phosphatase, albumin, and C-reactive protein (CRP) were measured in the patients using standard laboratory techniques. 


\section{Characterisation and potential diagnostic value of circulating matrix Gla-protein (MGP) species}

dp-ucMGP measurement

The method for dp-ucMGP measurement was a dual-antibody ELISA, with the capture antibody directed against the non-phosphorylated MGP sequence 3-15 (mAb-dpMGP; VitaK BV, Maastricht, the Netherlands) and the detecting antibody directed against the uncarboxylated MGP sequence 35-49 (mAb-ucMGP; VitaK BV). The same antibodies have been used previously for immunohistochemical staining [17, 39, 40].

The mAb-ucMGP was biotinylated using the EZ-Link Maleimide $\mathrm{PEO}_{2}$-Biotin kit (Pierce, Etten-Leur, the Netherlands) according to the manufacturer's instructions. The synthetic peptide Acetyl-SHESMESYELNPF-(AADO)-VQERIRERSKPVHELNREAC-amide, designated as $\mathrm{dpMGP}^{3-15}$-(AADO)-ucMGP ${ }^{35-54}$ (Prolmmune, Oxford, UK), was used as standard. The first 13 amino acid residues of this peptide were homologous to the non-phosphorylated MGP sequence 3-15 and the last 20 amino acid residues to the uncarboxylated MGP sequence 35-54. AADO indicates the linker 8-amino-3,6-dioxaoctanoic acid.

The mAb-dpMGP was diluted 200 -fold in carbonate buffer $(33 \mathrm{mmol} / \mathrm{l}$ disodiumcarbonate, $66 \mathrm{mmol} / \mathrm{l}$ sodiumbicarbonate, $\mathrm{pH} \mathrm{9.6)}$ and used for coating of the microtiter plate (100 $\mu \mathrm{l} /$ well). After incubation for 4 hours at room temperature, the remaining binding sites were blocked with $200 \mu$ l blocking buffer (2\% HNBSA, consisting of HEPES-NaCl buffer [ $25 \mathrm{mmol} / \mathrm{l}$ HEPES, $175 \mathrm{mmol} / \mathrm{I} \mathrm{NaCl}, \mathrm{pH}$ 7.7] and 2\% bovine serum albumin [BSA; Sigma, St.Louis, MO, USA]). The plate was incubated overnight at $4{ }^{\circ} \mathrm{C}$ and subsequently washed 4 times with 300 $\mu \mathrm{l}$ washing buffer (0.05\% Tween-20 in HEPES-NaCl buffer). Next, $90 \mu$ l of citrate plasma sample were diluted in $135 \mu \mathrm{l} 0.2 \%$ Protifar (Nutricia, Zoetermeer, the Netherlands) and the $\mathrm{dpMGP}^{3-15}$-(AADO)-ucMGP ${ }^{35-54}$ peptide was diluted 83,000 fold in $0.2 \%$ Protifar; $75 \mu \mathrm{l}$ of either solution were transferred to the microtiter plate. Subsequently, $25 \mu \mathrm{L}$ of the biotinylated mAb-ucMGP diluted 750 -fold in $0.2 \%$ Protifar were added to the plate and the plate was incubated with shaking $(150 \mathrm{rpm})$ overnight at $4{ }^{\circ} \mathrm{C}$. After 4 washing cycles with washing buffer, the plate was incubated with $100 \mu \mathrm{l}$ streptavidin-peroxide (Zymed, Breda, the Netherlands) diluted in HEPES-NaCl buffer and 0.5\% BSA during $30 \mathrm{~min}$ at room temperature. The plate was stained with $100 \mu \mathrm{l}$ of 3,3',5,5'tetramethylbenzidine (KLP, Gennep, the Netherlands) during 6-7 min. The staining process was stopped by adding $50 \mu \mathrm{l}$ of $1.0 \mathrm{M} \mathrm{H}_{2} \mathrm{SO}_{4}$, and the plate was read at $450 \mathrm{~nm}$. 


\section{dp-cMGP measurement}

The method for dp-cMGP measurement was also a dual-antibody ELISA, in which mAbdpMGP again served as a capture antibody. The detecting antibody was directed against the carboxylated MGP sequence 35-54 (mAb-cMGP; VitaK BV). The mAb-cMGP was biotinylated using the EZ-Link Maleimide $\mathrm{PEO}_{2}$-Biotin kit (Pierce) according to the manufacturer's instructions. The standard peptide for the dp-cMGP assay, designated as dpMGP ${ }^{3-}$ ${ }^{15}$-(AADO)-cMGP ${ }^{35-54}$ (Prolmmune), was a similar peptide as described for the dp-ucMGP assay, in which glutamate was replaced by $\gamma$-carboxyglutamate (Gla).

In brief, the mAb-dpMGP was diluted 200-fold in carbonate buffer and the mAb-cMGP 1500 times in $0.2 \%$ Protifar. The dpMGP ${ }^{3-15}$-(AADO)-cMGP ${ }^{35-54}$ synthetic peptide was diluted 83,000 fold in $0.2 \%$ in Protifar. From the standard or citrate plasma dilution ( $30 \mu \mathrm{l}$ of citrate plasma in $195 \mu \mathrm{l}$ of $0.2 \%$ Protifar), $25 \mu \mathrm{l}$ were added to the microtiter plate. The method was otherwise the same as described for the dp-ucMGP assay.

\section{t-ucMGP measurement}

The method for t-ucMGP measurement was a mono-antibody ELISA, as described previously [26]. The method has been modified, however, by using the new peptide dpMGP ${ }^{3-}$ ${ }^{15}$-(AADO)-ucMGP ${ }^{35-54}$ as standard. Biotinylated synthetic MGP homologous to the uncarboxylated MGP sequence 35-54 (ucMGP ${ }^{35-54}$ ) was used as tracer (Pepscan, Lelystad, the Netherlands). In brief, mAb-ucMGP was coupled to the microtiter plate. Serum sample or standard was supplemented with tracer, transferred to the microtiter plate, and incubated overnight at $4{ }^{\circ} \mathrm{C}$. The t-ucMGP concentration was calculated with the aid of a calibration curve of dpMGP ${ }^{3-15}$-(AADO)-ucMGP ${ }^{35-54}$. The intra-assay and inter-assay coefficients of variation (CV) have been found to be $8.9 \%$ and $11.4 \%$, respectively (Table 1 ).

\section{t-dpMGP measurement}

Circulating t-dpMGP levels were measured with the commercially available kit from Biomedica (Vienna, Austria). The kit is based on the competitive (mono-antibody) ELISA principle, with mAb-dpMGP coated on the microtiter plate. Biotinylated synthetic MGP homologous to the non-phosphorylated MGP sequence 3-15 (dpMGP ${ }^{3-15}$ ) was used as tracer, which was simultaneously added to the wells with standard, samples or controls. The t-dpMGP concentration was calculated using a calibration curve of synthetic dpMGP ${ }^{3-15}$. The intra-assay and inter-assay coefficients of variation (CV) have been found to be $10.6 \%$ and $9.6 \%$, respectively (Table 1 ). 


\section{Characterisation and potential diagnostic value of circulating matrix Gla-protein (MGP) species}

$\underline{\text { Statistical analysis }}$

Data are presented as mean $\pm \mathrm{SD}$, unless indicated otherwise. The RP was divided into 3 age groups: subjects aged $25-40 \mathrm{y}, 41-65 \mathrm{y}$, and 66-80 y. Tukey post-hoc and Kruskal-Wallis tests were used to test for differences between age-groups and patient populations. Student's $t$ test for independent samples, Mann-Whitney $U$ tests, the $X$-square test for homogeneity of proportions, and Fisher's exact test were used to test for differences between the patient populations and the concomitant healthy age groups. Student's $t$ test for paired samples and Wilcoxon's sign-rank test were used to examine within-group differences. Bivariate correlation analysis was performed using the Pearson and Spearman correlation coefficient. A $p$ value of $\leq 0.05$ was considered to be statistically significant; reported $p$ values are based on two-tailed tests of statistical significance. All statistical analyses were conducted using SPSS version 12.0 for Windows (SPSS Corp, Chicago, IL, USA). 
Table 1: Characteristics of the MGP assays

\begin{tabular}{|c|c|c|c|c|}
\hline Assay & dp-ucMGP & dp-cMGP & t-ucMGP & t-dpMGP \\
\hline & This paper & This paper & $\begin{array}{l}\text { Cranenburg et al, } \\
\text { J Vasc Res } 2008\end{array}$ & $\begin{array}{l}\text { Schurgers et al, } \\
\text { Clin Chim Acta } 2005\end{array}$ \\
\hline \multicolumn{5}{|l|}{ Reference values } \\
\hline $\begin{array}{l}\text { Healthy population } 25-80 \text { y } \\
\text { (mean } \pm S D \text {; this paper) }\end{array}$ & $447 \pm 188 \mathrm{pM}$ & $1763 \pm 478$ pM & $4704 \pm 1053$ nM & $14 \pm 3 \mathrm{nM}$ \\
\hline $\begin{array}{l}\text { Standard range } \\
\text { (according to manufacturer) }\end{array}$ & - & - & - & $0-90 \mathrm{nM}$ \\
\hline \multicolumn{5}{|l|}{ Materials and methods } \\
\hline Assay type & dual-antibody ELISA & dual-antibody ELISA & mono-antibody ELISA & mono-antibody ELISA \\
\hline \multirow[t]{9}{*}{ Antibodies } & capture antibody & capture antibody: & mAb-ucMGP & mAb-dpMGP \\
\hline & mAb-dpMGP & mAb-dpMGP & $\rightarrow$ uncarboxylated MGP & $\rightarrow$ non phosphorylated \\
\hline & $\rightarrow$ non-phosphorylated & $\rightarrow$ non-phosphorylated & Gla-domain residues & MGP residues 3-15 \\
\hline & MGP residues 3-15 & MGP residues 3-15 & $35-49$ & \\
\hline & Detecting antibody: & Detecting antibody: & & \\
\hline & biotinylated mAb-ucMGP & biotinylated mAb-cMGP & & \\
\hline & $\rightarrow$ uncarboxylated MGP & $\rightarrow$ carboxylated MGP & & \\
\hline & Gla-domain residues 35-49 & Gla-domain residues & & \\
\hline & & $35-54$ & & \\
\hline Tracer & - & - & biotinylated ucMGP & biotinylated MGP ${ }^{3-15}$ \\
\hline Standard & $\begin{array}{l}\text { synthetic } \\
\text { dpMGP }{ }^{3-15}-(A A D O)- \\
\text { ucMGP }^{35-54}\end{array}$ & $\begin{array}{l}\text { synthetic } \\
\text { dpMGP }{ }^{3-15} \text {-(AADO)- } \\
\text { cMGP }^{35-54}\end{array}$ & $\begin{array}{l}\text { synthetic } \\
\text { dpMGP }{ }^{3-15}-(A A D O)- \\
\text { ucMGP }^{35-54}\end{array}$ & synthetic MGP ${ }^{3-15}$ \\
\hline \multicolumn{5}{|l|}{ Assay characteristics } \\
\hline Intra-assay variation & $5.6 \%$ & $3.7 \%$ & $8.9 \%$ & $10.6 \%$ \\
\hline Inter-assay variation & $9.9 \%$ & $11.5 \%$ & $11.4 \%$ & $9.6 \%$ \\
\hline Lower detection limit & $21 \mathrm{pM}$ & $60 \mathrm{pM}$ & $98 \mathrm{nM}$ & $0.14 \mathrm{nM}$ \\
\hline Within-day variation & $5.5 \%$ & $7.4 \%$ & $10.8 \%$ & - (no circadian rhythm) \\
\hline Day-to-day variation & $8.4 \%$ & $13.1 \%$ & $11.3 \%$ & - (no circadian rhythm) \\
\hline \multicolumn{5}{|l|}{ Sample preparation } \\
\hline Matrix effect & $\begin{array}{l}\text { dp-ucMGP only } \\
\text { measurable in plasma }\end{array}$ & $\begin{array}{l}\text { dp-cMGP only } \\
\text { measurable in plasma }\end{array}$ & $\begin{array}{l}\text { Good correlation of } \\
\text { serum and citrate } \\
\text { plasma levels } \\
(r=0.897 ; p<0.001)\end{array}$ & $\begin{array}{l}\text { Concentration in } \\
\text { citrated plasma } \\
(18.7 \pm 4.3 \mathrm{nM}) \\
\text { high compared to } \\
\text { serum concentration } \\
(8.2 \pm 1.7 \mathrm{nM})\end{array}$ \\
\hline Freeze-thawing & $\begin{array}{l}\text { Up to } 8 \text { cycles stable } \\
\text { (11\% loss) }\end{array}$ & $\begin{array}{l}\text { Up to } 8 \text { cycles stable } \\
\text { ( } 9 \% \text { loss) }\end{array}$ & Up to 10 cycles stable & $\begin{array}{l}\text { Up to } 10 \text { cycles } \\
\text { stable ( } 17 \% \text { loss) }\end{array}$ \\
\hline $\begin{array}{l}\text { Storage at room } \\
\text { temperature }\end{array}$ & $\begin{array}{l}\text { Decrease of } 3 \% \text { after } \\
3 \text { hours; } 20 \% \text { after } \\
6 \text { hours }\end{array}$ & $\begin{array}{l}\text { Decrease of } 11 \% \text { after } \\
3 \text { hours; } 21 \% \text { after } \\
6 \text { hours }\end{array}$ & - & $\begin{array}{l}\text { Decrease of } 20 \% \\
\text { temperature after } \\
8 \text { hours }\end{array}$ \\
\hline \multicolumn{5}{|l|}{ Recommended } \\
\hline Type of sample & Citrate or EDTA plasma & Citrate or EDTA plasma & Serum or plasma & Serum or plasma * \\
\hline Centrifugation & $1,520 \mathrm{~g}$ & $1,520 \mathrm{~g}$ & $1,520 \mathrm{~g}$ & - \\
\hline Long-term storage & $-80^{\circ} \mathrm{C}\left(-112^{\circ} \mathrm{F}\right)$ & $-80^{\circ} \mathrm{C}\left(-112^{\circ} \mathrm{F}\right)$ & $-80^{\circ} \mathrm{C}\left(-112^{\circ} \mathrm{F}\right)$ & $-70^{\circ} \mathrm{C}(-94 \mathrm{~F})^{*}$ \\
\hline
\end{tabular}

Overview of the dual-antibody assays measuring desphosphorylated-uncarboxylated (dp-uc) MGP anddesphosphorylatedcarboxylated (dp-c) MGP, and the mono-antibody assays measuring total uncarboxylated (t-uc) MGP and total desphosphorylated (t-dp) MGP. In the section Antibodies, it is indicated by an arrow $(\rightarrow$ ) against which MGP residues the described antibodies were raised. The recommended method of sample preparation by the manufacturer is indicated by an asterisk (*). Abbreviations used: ELISA, enzyme-linked immunosorbent assay; mAb, monoclonal antibody. 


\section{Characterisation and potential diagnostic value of circulating matrix Gla-protein (MGP) species}

\section{Results}

\section{Assay characteristics}

The characteristics of the MGP assays are summarized in Table 1. The mean MGP levels of the total RP are given as reference values. We investigated the recovery after dilution for the newly-developed dp-ucMGP and dp-cMGP assays, by serial dilution of plasma from healthy subjects. For the dp-ucMGP assay, the optimal dilution of plasma was between 20 and $45 \%$ and for the dp-cMGP assay between 5 and 12\%. Spiking recovery was determined in a pooled citrate plasma sample from healthy subjects. The sample was spiked with synthetic ucMGP ${ }^{35-54}$ and dp-ucMGP levels were measured; the recovery was $80 \%$. The same was done for the dp-cMGP assay using the synthetic CMGP ${ }^{35-54}$ peptide; the recovery was $99 \%$. No significant differences in dp-ucMGP and dp-cMGP concentrations were found when comparing citrate, EDTA and heparin plasma, although slightly lower levels were measured in heparin plasma (data not shown). Circulating dp-ucMGP and dpcMGP levels were not measurable in serum.

\section{Circulating MGP levels}

Circulating MGP levels were measured in 75 healthy subjects of the RP and 146 patients, of whom the baseline characteristics are depicted in Tables $2 \mathrm{~A}$ and $2 \mathrm{~B}$, respectively. In the RP, there was a significant difference in the prevalence of hypertension and hypercholesterolemia between the 3 age groups. Among the patients, the AVD population was significantly older compared to the RD and ESRD patients. Cardiovascular risk factors (hypertension, hypercholesterolemia, and smoking) were highly prevalent in the AVD and ESRD patients. 
Table 2: Baseline characteristics of the healthy population (A) and the patient populations (B)

\begin{tabular}{|c|c|c|c|c|c|}
\hline \multirow[t]{2}{*}{ A Healthy reference population } & \multirow[t]{2}{*}{ Total population } & \multicolumn{3}{|c|}{ Age-group } & \multirow[t]{2}{*}{$\mathbf{p}$} \\
\hline & & $25-40$ years & $41-65$ years & $66-80$ years & \\
\hline $\mathrm{N}$ & 75 & 25 & 25 & 25 & \\
\hline \multicolumn{6}{|l|}{ Demographic characteristics } \\
\hline Gender $(\mathrm{m} / \mathrm{f})$ & $31 / 44$ & $13 / 12^{a}$ & $5 / 20^{b}$ & $13 / 12^{a}$ & 0.030 \\
\hline Age (years) & $53 \pm 17$ & $31 \pm 4^{a}$ & $58 \pm 6^{b}$ & $70 \pm 3^{c}$ & $<0.001$ \\
\hline $\mathrm{BMI}\left(\mathrm{kg} / \mathrm{m}^{2}\right)$ & $26 \pm 3$ & $24 \pm 3^{a}$ & $27 \pm 3^{b}$ & $26 \pm 2^{b}$ & $<0.001$ \\
\hline \multicolumn{6}{|l|}{ Clinical characteristics } \\
\hline Hypertension (number [\%]) & $15(20)$ & $1(4)^{a}$ & $5(20)^{b}$ & $9(36)^{b}$ & 0.005 \\
\hline \multicolumn{6}{|l|}{ Hypercholesterolemia } \\
\hline (number [\%]) & $7(9)$ & $0(0)^{a}$ & $2(8)^{a b}$ & $5(20)^{b}$ & 0.050 \\
\hline Current smoking (number [\%]) & $7(9)$ & $1(4)$ & $5(20)$ & $1(4)$ & ns \\
\hline DM (number [\%]) & $0(0)$ & $0(0)$ & $0(0)$ & $0(0)$ & ns \\
\hline CVD (number [\%]) & $0(0)$ & $0(0)$ & $0(0)$ & $0(0)$ & ns \\
\hline B Patient population & RD & AVD & ESRD & p & \\
\hline $\mathrm{N}$ & 51 & 50 & 45 & & \\
\hline \multicolumn{6}{|l|}{ Demographic characteristics } \\
\hline Gender $(\mathrm{m} / \mathrm{f})$ & $27 / 24^{a}$ & $9 / 11^{b}$ & $20 / 25^{a}$ & 0.002 & \\
\hline Age (years) & $56 \pm 12^{a}$ & $70 \pm 10^{b}$ & $59 \pm 16^{a}$ & $<0.001$ & \\
\hline BMI $\left(\mathrm{kg} / \mathrm{m}^{2}\right)$ & $27 \pm 6^{a}$ & $28 \pm 4^{b}$ & $26 \pm 6^{a}$ & 0.005 & \\
\hline \multicolumn{6}{|l|}{ Clinical characteristics } \\
\hline Hypertension (number [\%]) & $17(32)^{a}$ & $43(86)^{b}$ & $40(89)^{b}$ & $<0.001$ & \\
\hline \multicolumn{6}{|l|}{ Hypercholesterolemia } \\
\hline (number $[\%]$ ) & $9(18)^{a}$ & $36(72)^{b}$ & Unknown & $<0.001$ & \\
\hline Current smoking (number [\%]) & $8(16)^{a}$ & $25(50)^{b}$ & $5(11)^{a}$ & $<0.001$ & \\
\hline DM (number [\%]) & $0(0)^{a}$ & $10(20)^{b}$ & $6(13)^{b}$ & 0.005 & \\
\hline CVD (number [\%]) & $0(0)^{a}$ & $50(100)^{b}$ & $17(38)^{c}$ & $<0.001$ & \\
\hline
\end{tabular}

Demographic and clinical characteristics of the healthy population according to age groups (25-40,41-65, and 66-80 years; A) and of the rheumatic disease (RD), aortic valve disease (AVD), and end-stage renal disease (ESRD) patients (B). Data are presented either as mean \pm SD or as numbers, with percentage of total between parentheses. $P$ values for overall comparisons between healthy age-groups (A) and patient populations (B) are given as well as significant differences between 2 groups or populations, which are depicted by superscripts $a, b$, $c,(p<0.05)$. Abbreviations used: BMI, body mass index; DM, diabetes mellitus (use of insulin or oral antidiabetic agents); CVD, cardiovascular disease (a history of coronary artery disease [myocardial infarction, angina pectoris or evidence of obstructive disease by angiography], cerebrovascular disease [thrombotic stroke or transient ischemic attack], calcific aortic valve disease, or peripheral artery disease [a history of claudication or lower extremity revascularization]). 


\section{Characterisation and potential diagnostic value of circulating matrix Gla-protein (MGP) species}

In Figure 1, the circulating concentrations of the various MGP species in the RP (according to age group) and in the patient populations are depicted. MGP levels in the RD and ESRD patients were compared with those in the concomitant healthy age group 41-65 y, and MGP levels in the AVD patients with those in the age group 66-80 y. In the RP, there was no difference in MGP levels between men and women. The dp-ucMGP and dp-cMGP levels significantly correlated with age in the $\operatorname{RP}(r=0.263, p=0.022$ and $r=0.714, p<0.001$, respectively).

Figure 1: Circulating MGP levels in the reference and patient populations

A

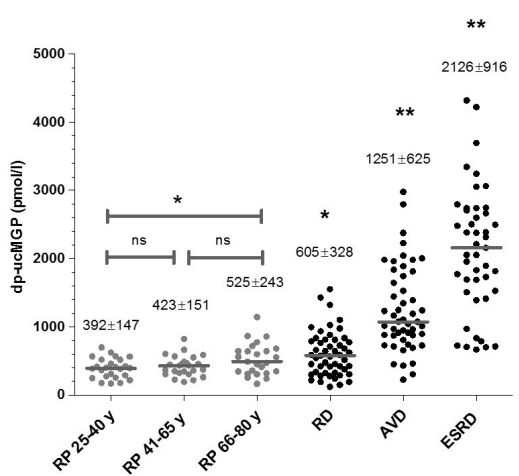

C

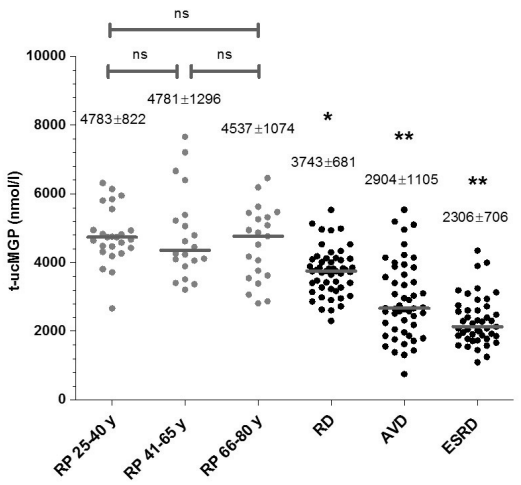

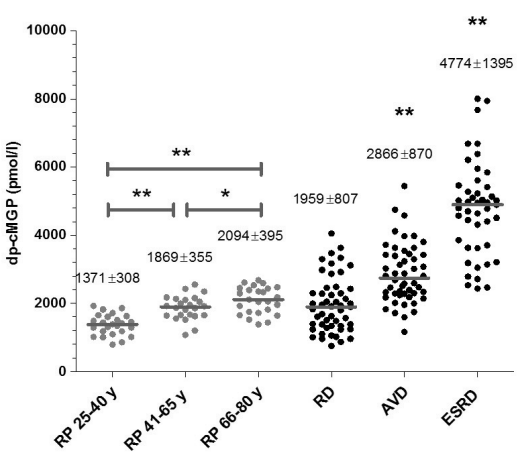

D

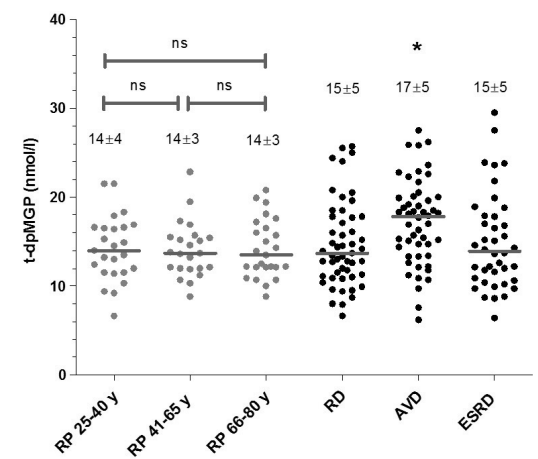

Circulating dp-ucMGP (A), dp-cMGP (B), t-ucMGP (C), and t-dpMGP (D) levels in the refence population (RP, $n=75$ ) according to age-group (25-40, 41-65, 66-80 years) and in the rheumatic disease (RD, $n=51)$, aortic valve disease (AVD, $n=50)$, and end-stage renal disease (ESRD, $n=45$ ) patient populations. Median MGP levels are depicted as horizontal bars and mean \pm SD MGP levels are given for each age group and patient population.

$*$ : $p<0.05, * *$ : $p<0.001$ ns: non significant. 
The dp-ucMGP levels (Figure 1A) in the RP were highest in the oldest age group. In all patient populations, dp-ucMGP levels were significantly higher than in the healthy group of comparable age. Particularly in the AVD and ESRD patients, very high dp-ucMGP levels were measured; mean dp-ucMGP levels were 2.4 and 5.0 times higher than in the corresponding age group, respectively. The dp-cMGP levels (Figure 1B) significantly increased with age in the RP. The dp-cMGP levels in AVD and ESRD patients were 1.4 and 2.6 times as high as those in the corresponding age-group, respectively. In the RD patients, dpcMGP levels were not significantly different compared to the healthy age group 41-65 y. The t-ucMGP levels (Figure 1C) were independent of age in the RP. All patient populations had significantly lower ucMGP levels. The t-dpMGP levels (Figure 1D) were significantly higher in AVD patients compared to the healthy age-group 66-80 y. Otherwise, there were no significant differences.

\section{MGP and clinical characteristics in patients}

Among the total patient population $(n=146)$, there was no significant correlation between circulating MGP and age. There was no difference between men and women, smoking and non-smoking patients, and patients with and without DM (data not shown).

Figure 2 shows the distribution of the MGP levels in the RD groups (CC, gout, DISH, and SLE) and in healthy subjects of the same age (41-65 y). Patients with gout had significantly higher dp-ucMGP levels (Figure 2A). Some individual RA and SLE patients had high dpucMGP levels (>700 pmol/l), but on a group level this was not significant. The dp-cMGP levels (Figure $2 \mathrm{~B}$ ) in the RD groups did not differ significantly from the healthy subjects, although again some RA patients had high dp-cMGP levels ( $>3000 \mathrm{pmol} / \mathrm{l})$. Patients with $\mathrm{CC}$ had slightly lower t-ucMGP levels (Figure $2 \mathrm{C}$ ) compared with the healthy subjects. The t-dpMGP levels (Figure 2D) in the RD groups were comparable with those of the healthy subjects. Unfortunately, only 4 DISH patients could be included, for which reliable statistical analysis could not be performed. Figure 2 shows that in general the MGP levels of these patients were comparable with those of healthy subjects. The range of dp-ucMGP levels in the DISH patients was relatively large (120-708 pmol/I). 


\section{Characterisation and potential diagnostic value of circulating matrix Gla-protein (MGP) species}

Figure 2: Circulating MGP levels in the rheumatic disease (RD) patient population
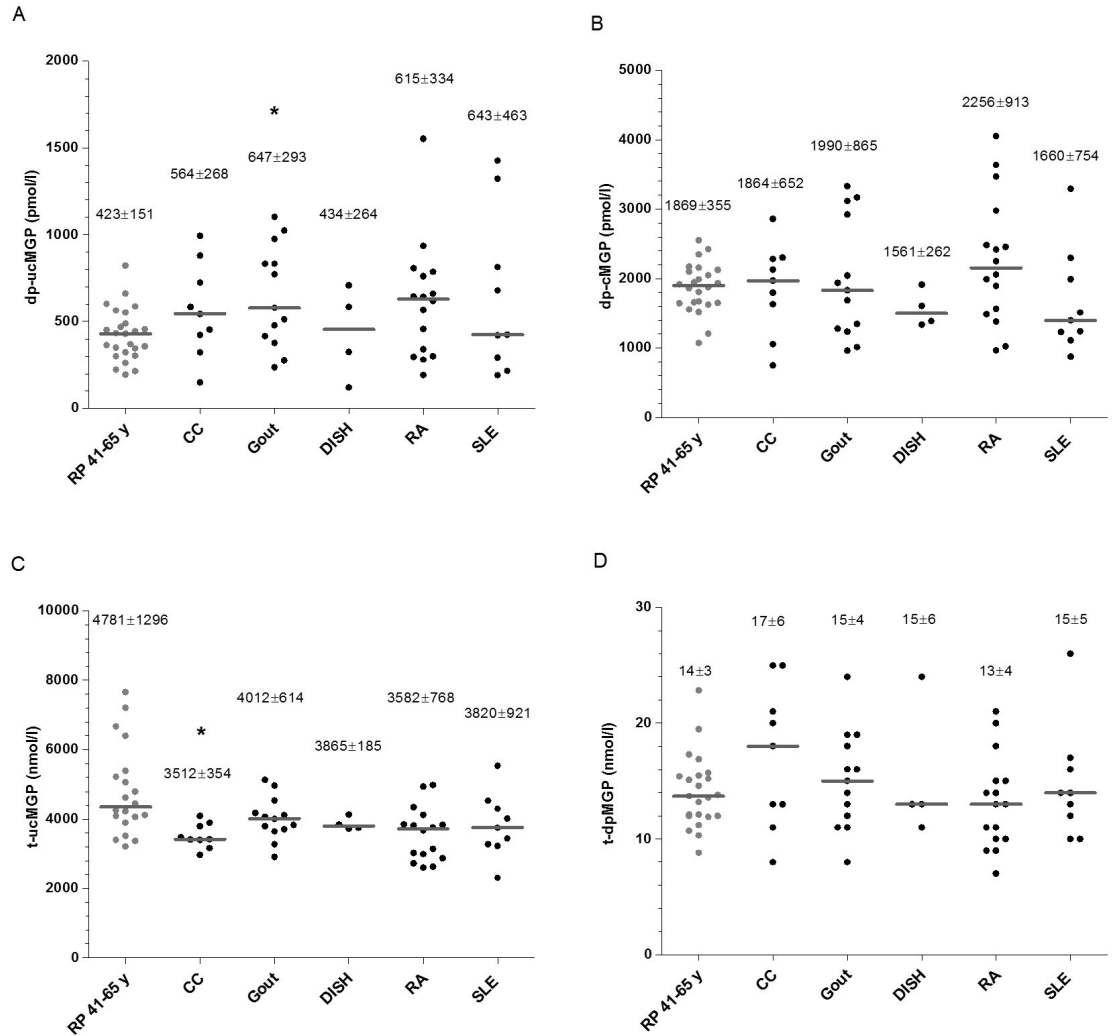

Circulating dp-ucMGP (A), dp-cMGP (B), t-ucMGP (C), and t-dpMGP (D) levels in RD patients with chondrocalcinosis (CC, $n=9)$, gout $(n=13)$, diffuse idiopathic skeletal hyperostosis (DISH, $n=4)$, rheumatoid arthritis $(R A, n=16)$, and systemic lupus erythematosus (SLE, $n=9$ ). For reference, MGP values in healthy subjects of comparable age (RP 41-65 years, $n=25$ ) are depicted. Median MGP levels are depicted as horizontal bars and mean \pm SD MGP levels are given for each group. ${ }^{*}: p<0.05,{ }^{* *}: p<0.001$.

In 33 patients of the AVD population, there were no significant differences in MGP levels between those with aortic valve sclerosis $(n=9)$, mild $(n=6)$, and moderate to severe $(n=18)$ aortic stenosis (data not shown). Circulating dp-ucMGP and dp-cMGP positively correlated with age $(r=0.401, p=0.004$ and $r=0.403, p=0.004$, respectively) in the AVD population. This was not seen in ESRD patients. There was also no correlation between circulating MGP and dialysis vintage in these patients. Circulating dp-ucMGP positively correlated with the calcium-phosphate product in ESRD patients $(r=0.473, p=0.006)$. 


\section{Vitamin K status}

In the both VKS groups (MK-7 and MK-4 supplementation), dp-ucMGP levels (Figure 3A) were significantly lower after supplementation. Remarkably, also the dp-cMGP levels (Figure 3B) were slightly lower after MK7 supplementation. The decrease in dp-cMGP levels was even more pronounced in the subjects receiving MK-4, which might be due to the fact that MK-4 was given in a much higher dose and 3 months longer than MK-7. Circulating t-ucMGP levels (Figure $3 \mathrm{C}$ ) remained the same in all groups, indicating that these are not influenced by changes in systemic vitamin $\mathrm{K}$ status. Circulating t-dpMGP levels (Figure 3D) were significantly increased in the MK-7 group, but remained unchanged in the MK-4 group. In the placebo-group, all MGP levels were unchanged after 6 months with exception of the t-dpMGP levels, which were slightly increased.

The MGP measurements in the healthy subjects receiving VKA for 4 weeks are also depicted in Figure 3. The dp-ucMGP levels (Figure $3 A$ ) increased in all 17 subjects, and were 1.6 to 5.3 times higher after VKA. The dp-cMGP (Figure 3B) and t-ucMGP (Figure 3C) levels remained unchanged. There was a significant increase in t-dpMGP levels after 4 weeks (Figure 3D). 


\section{Characterisation and potential diagnostic value of circulating matrix Gla-protein (MGP) species}

Figure 3: Circulating MGP levels before and after vitamin K supplementation (VKS) and vitamin K antagonists (VKA) treatment

Circulating dp-ucMGP (A), dp-cMGP (B), t-ucMGP (C), and t-dpMGP (D) levels in healthy subjects before and after supplementation with menaquinone-7 for 3 months (MK-7, n=24), with menaquinone-4 (MK-4, n=28) or placebo $(n=11)$ for 6 months, and after treatment with VKA for 4 weeks $(n=17)$. Median MGP levels are depicted as horizontal bars and mean \pm SD MGP levels are given for each group. $*$ : $p<0.05, * *: p<0.001$.

A
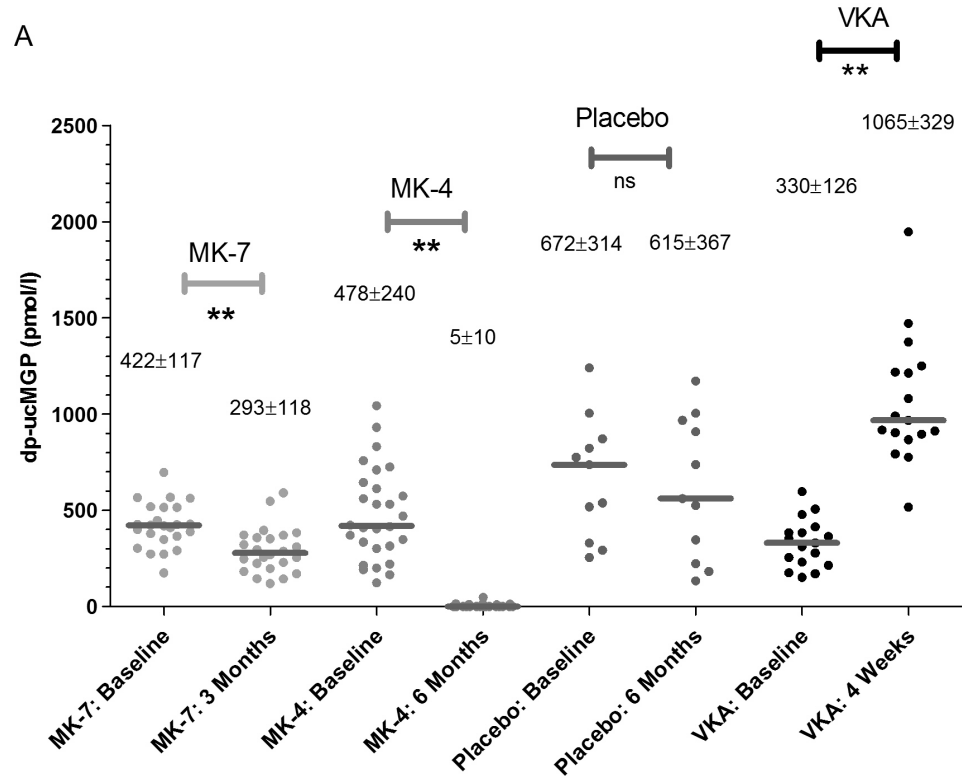

B

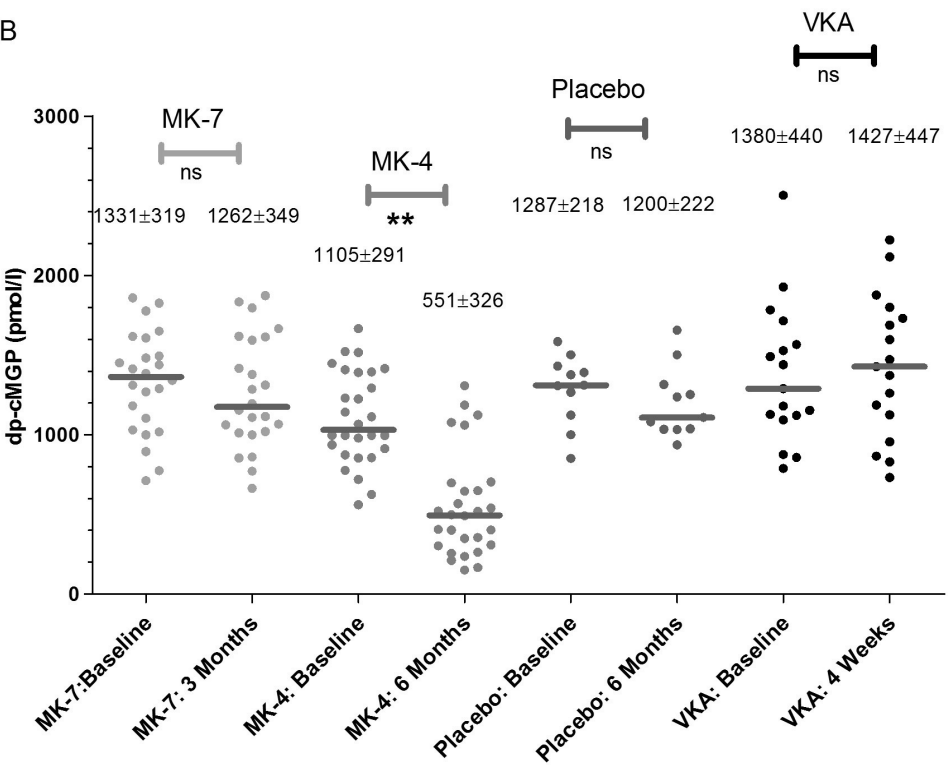



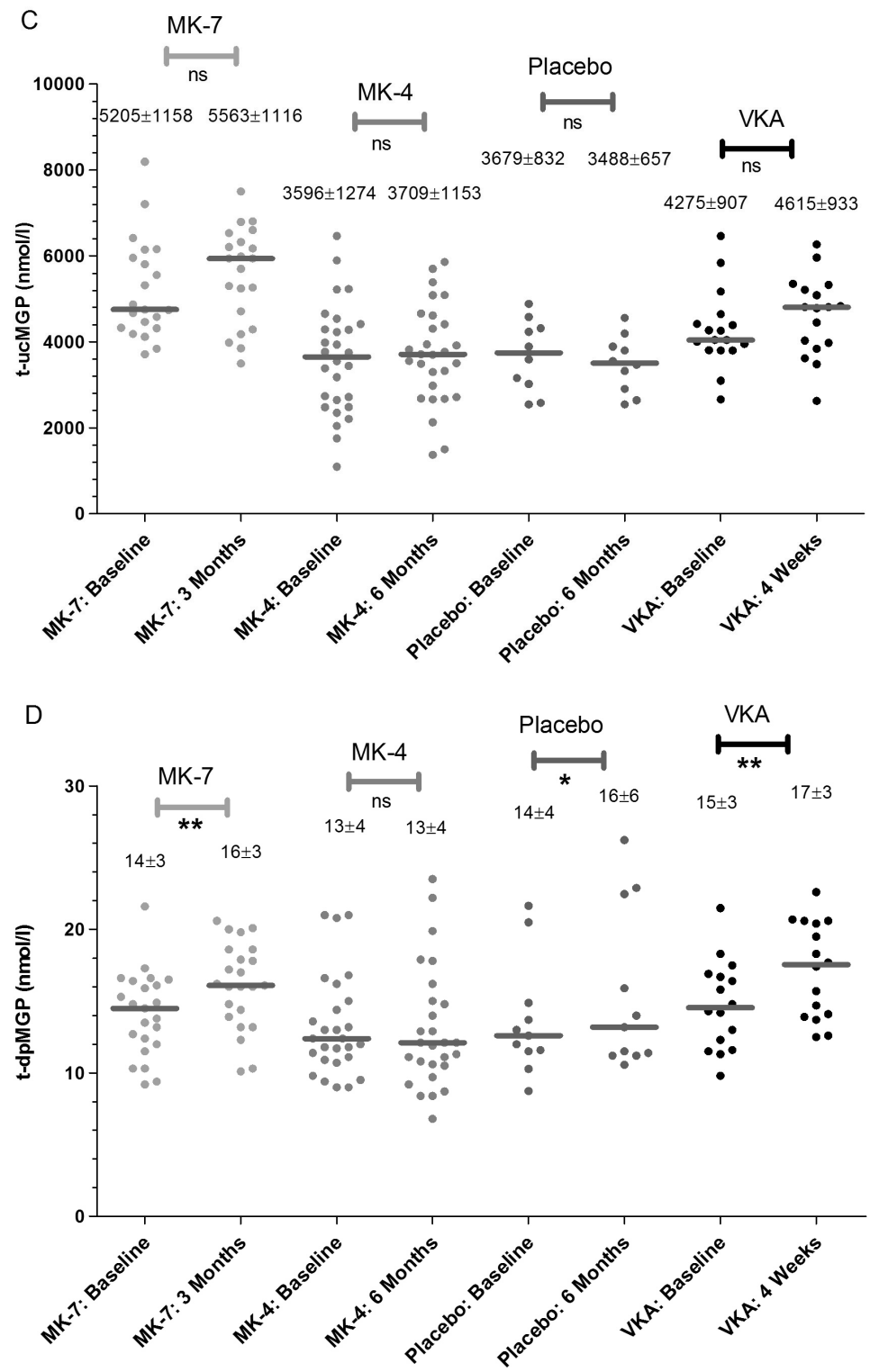


\section{Characterisation and potential diagnostic value of circulating matrix Gla-protein (MGP) species}

\section{Discussion}

Measuring MGP levels in the circulation may be valuable in two different settings: assessing the amount of vascular damage and the vascular vitamin $\mathrm{K}$ status. In this paper, we report on the comparison of four different MGP assays: the available mono-antibody $t$ ucMGP and t-dpMGP assays, and the novel dual-antibody dp-ucMGP and dp-cMGP assays. Each assay detects a different fraction of the total circulating MGP pool, which explains the widely different results when used in patient populations (Table 3 ). This is illustrated by the finding of both high (dp-ucMGP and dp-cMGP) and low (t-ucMGP) MGP levels in patients with AVD and ESRD compared to healthy subjects of the same age. Another major difference between the assays was the response to changes in systemic vitamin $\mathrm{K}$ status, for which both the dp-ucMGP and dp-cMGP assays were sensitive. Particularly the dp-ucMGP levels showed a fast response to both VKS (both MK-7 and MK4 supplementation) and VKA (4 week-treatment with acenocoumarol). Circulating dp-ucMGP is clearly a marker capable of detecting changes in vitamin $\mathrm{K}$ status.

The measurement of circulating dp-ucMGP confirms the previous reported finding of uncarboxylated, inactive MGP species in the circulation of healthy subjects [26]. This has also been reported for osteocalcin, a vitamin K-dependent protein synthesized in bone, of which uncarboxylated levels are also present in the circulation of healthy subjects. Measurements of circulating uncarboxylated and carboxylated osteocalcin are commonly used as a marker for vitamin $\mathrm{K}$ status of bone $[41,42]$. The dp-ucMGP levels decreased to almost immeasurably low levels after 6 months of VKS, indicating that the even in healthy subjects the vascular vitamin $\mathrm{K}$ status is insufficient for maximal MGP carboxylation. It is currently not known why also dp-cMGP levels decreased significantly after 6 months of MK-4 supplementation. The use of a ratio of uncarboxylated and carboxylated MGP may not be useful to assess vascular vitamin $K$ status, since the ratio will not change upon long-term VKS as both dp-ucMGP and dp-cMGP levels decrease.

Vascular vitamin $\mathrm{K}$ deficiency may result in an increased risk for arterial calcification. Indeed, a low dietary intake of menaquinones was associated with increased cardiovascular mortality and arterial calcification in several population studies [20, 21, 43]. In animal models it has been a consistent finding that treatment with VKA (coumarin derivatives) results in extensive arterial calcification $[8,39]$. Moreover, patients treated with coumarin were reported to have significantly more coronary artery and valve calcifications compared to those not treated with anticoagulation [44-46]. The newly-developed dp-ucMGP assay enables investigation of the potential correlation between vascular vitamin $\mathrm{K}$ status and (cardiovascular) risk, disease, or even mortality. 
In the present study, the AVD and ESRD patients had very high circulating dp-ucMGP levels, indicating a poor vascular vitamin K status. Preliminary results of two large AVD and ESRD patient cohorts show that high dp-ucMGP levels predict mortality in these patients [47].

The RD patient population was included to assess whether patients with an increased risk for cardiovascular calcification without CVD (RA and SLE), and patients with non-vascular pathologies (CC, DISH, gout), would have different circulating MGP levels. Overall, patients with RD had significantly higher dp-ucMGP levels and significantly lower ucMGP levels. In the individual patient populations, these results were seen in patients with gout and CC, respectively. However, the differences were very small and the influence of cartilage disease on circulating MGP levels seems to be minimal compared to that of CVD. Patients with SLE and RA did not have significantly different circulating MGP levels, indicating that a poor vascular vitamin $\mathrm{K}$ status might not contribute to the increased arterial calcification risk in these patients.

The performance of the dp-ucMGP and dp-cMGP assays, the first dual-antibody MGP assays, was satisfactory with respect to intra- and inter-assay variation, stability upon repeated thawing and freezing cycles, and storage at room temperature during several hours. An important finding was that dp-ucMGP and dp-cMGP levels could not be measured in serum. Incubation of full-length synthetic MGP with thrombin, a circulating serineprotease, resulted in four MGP fragments as analyzed with mass-spectrometry (T. Hackeng et al, personal communication). MGP therefore appears to have several cleavage sites for thrombin, and might be degraded by the thrombin generated during serum preparation, resulting in non-measurable serum dp-ucMGP and dp-cMGP levels. More research is needed to confirm this hypothesis.

The method for t-ucMGP measurement has been improved by using a new synthetic standard, which is the same as used in the dp-ucMGP assay enabling comparison between the assays. Therefore, the t-ucMGP levels were found to be higher as compared to levels previously measured [26], but the differences in circulating t-ucMGP levels between healthy subjects, AVD, and ESRD patients have remained the same. Previously, we have demonstrated that circulating t-ucMGP is inversely correlated with the extent of coronary artery calcification in HDpatients [27]. Circulating t-ucMGP may be an important biomarker for prevalent vascular calcification in high-risk subjects. It can be measured both in serum and plasma [26] and it has the additional advantage of being virtually uninfluenced by systemic vitamin $\mathrm{K}$ status, as demonstrated in the present study. 


\section{Characterisation and potential diagnostic value of circulating matrix Gla-protein (MGP) species}

The results of MGP measurements with the now commercially available t-dpMGP assay showed that there was a large overlap between t-dpMGP levels of healthy subjects and patients. In the past, low as well as high t-dpMGP levels have been reported in patients with atherosclerotic disease [25, 30]. Circulating t-dpMGP levels increased significantly after MK-7 supplementation, after coumarin treatment and also in the placebo group. The inconsistency of $\mathrm{t}$-dpMGP results, together with the large overlap between patient and reference values, indicates that this assay is not capable of identifying patient populations and would not be suited for individual patient diagnosis.

The present study was primarily designed to compare the available MGP assays and to characterize the newly-developed MGP assays. It is therefore less suited to assess full clinical value of the individual MGP assays, which will require much further study. Another limitation was the inability to measure other circulating MGP species, including circulating phosphorylated MGP levels, which could potentially clarify the results obtained with the dp-ucMGP and dp-cMGP assay. We are aiming to develop measurements for phosphorylated MGP, but it has proven to be technically difficult synthesize the relevant MGP sequence 3-15 in its phosphorylated form and to develop monoclonal antibodies against it. Therefore, there is no assay available to measure this fraction of MGP at this moment.

Not only the phosphorylation state of MGP, but also the synthesis and degradation of MGP may contribute to the variations in concentrations of MGP species within the patient populations. Measurements with both mono-antibody assays resulted in MGP levels within the nanomolar ranger, whereas with both dual-antibody assays levels within the picomolar range were measured. An explanation for the observed differences may be the breakdown of MGP into fragments. Although we have previously shown for the monoantibody t-ucMGP assay that it is able to detect full-length MGP [26], fragments of MGP could in theory also contribute to measurements of MGP with the mono-antibody assays. It is less likely that the dual-antibody MGP assays detect MGP fragments, since these assays are based on two antibodies directed against residues in the $\mathrm{N}$-terminal region (315) and in the central region of MGP (35-54). This may result in higher MGP levels measured with the mono-antibody assays. However, further fundamental research into the processes of MGP synthesis, transport, carboxylation, and phosphorylation is necessary to explain the results of the circulating MGP measurements on a cellular level. 
In conclusion, we compared four different assays to measure circulating MGP, including the first dual-antibody assays. Based on our results, each assay may have its own application for research and/or for diagnostic use. From these assays, the dp-ucMGP assay seems to be particularly suited to assess vascular vitamin $\mathrm{K}$ status, and may therefore contribute to cardiovascular risk assessment. Further research is necessary to investigate the full diagnostic potential of the different MGP assays. 


\section{Characterisation and potential diagnostic value of circulating matrix Gla-protein (MGP) species}

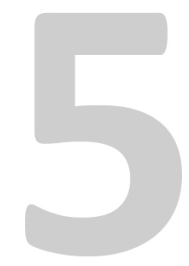

\section{Acknowledgements}

The authors thank dr. E. Theuwissen for critical reading of the manuscript.

\section{References}

1. Schurgers, L.J., E.C.M. Cranenburg, and C. Vermeer, Matrix Gla-protein: The calcification inhibitor in need of vitamin K. Thromb Haemost, 2008. 100(4): p. 593-603.

2. El-Maadawy, S., et al., Cartilage formation and calcification in arteries of mice lacking matrix Gla protein. Connect Tissue Res, 2003. 44 Suppl 1: p. 272-8.

3. Luo, G., et al., The matrix Gla protein gene is a marker of the chondrogenesis cell lineage during mouse development. J Bone Miner Res, 1995. 10(2): p. 325-34.

4. Wallin, R., D. Cain, and D.C. Sane, Matrix Gla protein synthesis and gamma-carboxylation in the aortic vessel wall and proliferating vascular smooth muscle cells--a cell system which resembles the system in bone cells. Thromb Haemost, 1999. 82(6): p. 1764-7.

5. Price, P.A., J.S. Rice, and M.K. Williamson, Conserved phosphorylation of serines in the Ser-X-Glu/Ser(P) sequences of the vitamin K-dependent matrix Gla protein from shark, lamb, rat, cow, and human. Protein Sci, 1994. 3(5): p. 822-30.

6. Price, P.A., M.R. Urist, and Y. Otawara, Matrix Gla protein, a new gamma-carboxyglutamic acid-containing protein which is associated with the organic matrix of bone. Biochem Biophys Res Commun, 1983. 117(3): p. 765-71.

7. Price, P.A. and M.K. Williamson, Primary structure of bovine matrix Gla protein, a new vitamin K-dependent bone protein. J Biol Chem, 1985. 260(28): p. 14971-5.

8. Price, P.A., S.A. Faus, and M.K. Williamson, Warfarin causes rapid calcification of the elastic lamellae in rat arteries and heart valves. Arterioscler Thromb Vasc Biol, 1998. 18(9): p. 1400-7.

9. Price, P.A., et al., The elastic lamellae of devitalized arteries calcify when incubated in serum: evidence for a serum calcification factor. Arterioscler Thromb Vasc Biol, 2006. 26(5): p. 1079-85.

10. Wallin, R., et al., Modulation of the binding of matrix Gla protein (MGP) to bone morphogenetic protein-2 (BMP-2). Thromb Haemost, 2000. 84(6): p. 1039-44.

11. Bostrom, K., et al., Matrix GLA protein modulates differentiation induced by bone morphogenetic protein-2 in C3H10T1/2 cells. J Biol Chem, 2001. 276(17): p. 14044-52.

12. Zebboudj, A.F., M. Imura, and K. Bostrom, Matrix GLA protein, a regulatory protein for bone morphogenetic protein-2. J Biol Chem, 2002. 277(6): p. 4388-94.

13. Wallin, R., L.J. Schurgers, and N. Wajih, Effects of the blood coagulation vitamin $K$ as an inhibitor of arterial calcification Thromb Res 2008. 122(3): p. 411-7.

14. Wajih, N., et al., Processing and transport of matrix gamma-carboxyglutamic acid protein and bone morphogenetic protein-2 in cultured human vascular smooth muscle cells: evidence for an uptake mechanism for serum fetuin. J Biol Chem, 2004. 279(41): p. 43052-60.

15. Price, P.A., S.A. Faus, and M.K. Williamson, Warfarin-induced artery calcification is accelerated by growth and vitamin D. Arterioscler Thromb Vasc Biol, 2000. 20(2): p. 317-27.

16. Sweatt, A., et al., Matrix Gla protein (MGP) and bone morphogenetic protein-2 in aortic calcified lesions of aging rats. J Thromb Haemost, 2003. 1(1): p. 178-85.

17. Schurgers, L.J., et al., Novel conformation-specific antibodies against matrix gamma-carboxyglutamic acid (Gla) protein: undercarboxylated matrix Gla protein as marker for vascular calcification. Arterioscler Thromb Vasc Biol, 2005. 25(8): p. 1629-33. 
18. Murshed, M., et al., Extracellular matrix mineralization is regulated locally; different roles of two glacontaining proteins. J Cell Biol, 2004. 165(5): p. 625-30.

19. Holden, R.M. and S.L. Booth, Vascular calcification and chronic kidney diseases; the role of vitamin K. . Nat Clin Pract Nephrol, 2007. 3(10): p. 522-3.

20. Beulens, J.W., et al., High dietary menaquinone intake is associated with reduced coronary calcification. Atherosclerosis, 2009. 203(2): p. 489-93.

21. Gast, G.C., et al., A high menaquinone intake reduces the incidence of coronary heart disease. Nutr Metab Cardiovasc Dis, 2009. 19(7): p. 504-10.

22. Shearer, M.J. and P. Newman, Metabolism and cell biology of vitamin K. Thromb Haemost, 2008. 100(4): p. 530-47.

23. Schurgers, L.J., et al., Vitamin K-containing dietary supplements: comparison of synthetic vitamin $K 1$ and natto-derived menaquinone-7. Blood, 2007. 109(8): p. 3279-83.

24. Otawara, Y.Y. and P.P.A. Price, Developmental appearance of matrix GLA protein during calcification in the rat. J Biol Chem, 1986. 261(23): p. 10828-32.

25. Schurgers, L.J., et al., Characteristics and performance of an immunosorbent assay for human matrix Glaprotein. Clin Chim Acta, 2005. 351(1-2): p. 131-8.

26. Cranenburg, E.C., et al., The circulating inactive form of Matrix Gla Protein (UCMGP) as a biomarker for cardiovascular calcification J Vasc Res 2008. 45: p. 427-36.

27. Cranenburg, E.C.M., et al., Uncarboxylated matrix Gla protein (UcMGP) is associated with coronary artery calcification in haemodialysis patients. Thromb Haemost, 2009. 101(2): p. 359-66.

28. Koos, R., et al., Relation of circulating Matrix Gla-Protein and anticoagulation status in patients with aortic valve calcification. Thromb Haemost, 2009. 101(4): p. 706-13.

29. Parker, B.D., et al., Association of kidney function and uncarboxylated matrix Gla protein: Data from the Heart and Soul Study. Nephrol Dial Transplant, 2009. 24(7): p. 2095-2101.

30. Braam, L.A., et al., Assay for human matrix gla protein in serum: potential applications in the cardiovascular field. Arterioscler Thromb Vasc Biol, 2000. 20(5): p. 1257-61.

31. Rosenhek, R., et al., Predictors of outcome in severe, asymptomatic aortic stenosis. N Engl J Med, 2000. 343(9): p. 611-7.

32. Blacher, J., et al., Arterial calcifications, arterial stiffness, and cardiovascular risk in end-stage renal disease. Hypertension, 2001. 38(4): p. 938-42.

33. London, G.M., et al., Arterial media calcification in end-stage renal disease: impact on all-cause and cardiovascular mortality. Nephrol Dial Transplant, 2003. 18(9): p. 1731-40.

34. Asanuma, Y., et al., Premature coronary-artery atherosclerosis in systemic lupus erythematosus. N Engl J Med, 2003. 349(25): p. 2407-15.

35. Wang, S., et al., Prevalence and extent of calcification over aorta, coronary and carotid arteries in patients with rheumatoid arthritis. J Intern Med, 2009. 266(5): p. 445-52.

36. Chung, C.P., et al., Increased coronary-artery atherosclerosis in rheumatoid arthritis: relationship to disease duration and cardiovascular risk factors. Arthritis Rheum, 2005. 52(10): p. 3045-53.

37. Knapen, M.H., L.J. Schurgers, and C. Vermeer, Vitamin $K(2)$ supplementation improves hip bone geometry and bone strength indices in postmenopausal women. Osteoporos Int, 2007. 18(7): p. 963-72.

38. Bonow, R.O., et al., ACC/AHA 2006 guidelines for the management of patients with valvular heart disease: a report of the American College of Cardiology/American Heart Association Task Force on Practice Guidelines (writing Committee to Revise the 1998 guidelines for the management of patients with valvular heart disease) developed in collaboration with the Society of Cardiovascular Anesthesiologists endorsed by the Society for Cardiovascular Angiography and Interventions and the Society of Thoracic Surgeons. J Am Coll Cardiol, 2006. 48(3): p. 1-148.

39. Schurgers, L.J., et al., Regression of warfarin-induced medial elastocalcinosis by high intake of vitamin $K$ in rats. Blood, 2007. 109(7): p. 2823-31. 


\section{Characterisation and potential diagnostic value of circulating matrix Gla-protein (MGP) species}

40. Shroff, R.C., et al., Dialysis accelerates medial vascular calcification in part by triggering smooth muscle cell apoptosis. Circulation, 2008. 118(17): p. 1748-57.

41. Sokoll, L.J., et al., Changes in serum osteocalcin, plasma phylloquinone, and urinary gammacarboxyglutamic acid in response to altered intakes of dietary phylloquinone in human subjects. Am J Clin Nutr, 1997. 65(3): p. 779-84.

42. Sokoll, L.J. and J.A. Sadowski, Comparison of biochemical indexes for assessing vitamin $K$ nutritional status in a healthy adult population. Am J Clin Nutr, 1996. 63(4): p. 566-73.

43. Geleijnse, J.M., et al., Dietary intake of menaquinone is associated with a reduced risk of coronary heart disease: the Rotterdam Study. J Nutr, 2004. 134(11): p. 3100-5.

44. Schurgers, L.J., et al., Oral anticoagulant treatment: friend or foe in cardiovascular disease? Blood, 2004. 104(10): p. 3231-2.

45. Koos, R., et al., Relation of oral anticoagulation to cardiac valvular and coronary calcium assessed by multislice spiral computed tomography. Am J Cardiol, 2005. 96(6): p. 747-9.

46. Lerner, R.G., et al., Warfarin use and the risk of valvular calcification. J Thromb Haemost, 2009. 7: p. 202327.

47. Schurgers, L.J., et al., The circulating inactive form of matrix gla protein is a surrogate marker for vascular calcification in chronic kidney disease: a preliminary report. Clin J Am Soc Nephrol, 2010. 5(4): p. 568-75. 



\section{CHAPTER 6}

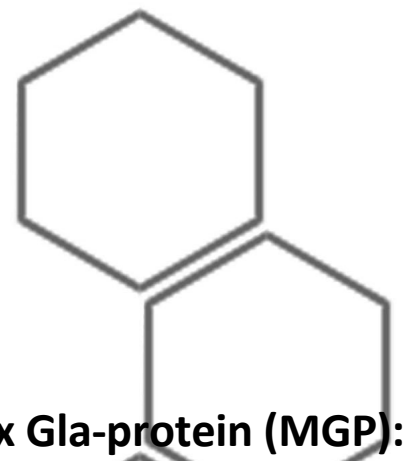

a new case of Keutel syndrome serves as a model

for exploring MGP functions in humans

Rare mutations in the gene encoding Matrix Gla-protein (MGP):

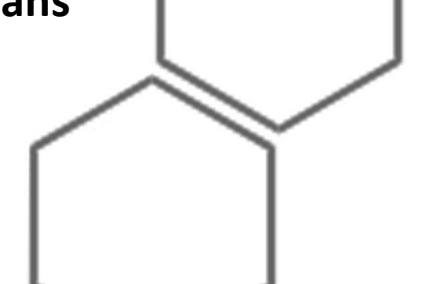

Ellen C.M. Cranenburg ${ }^{1 *}$, Karin Y. van Spaendonck-Zwarts ${ }^{2 *}$, Luisa Bonafe ${ }^{3}$, Laureane Mittaz Crettol ${ }^{3}$, Lars A. Rödiger ${ }^{4}$, Freek G. Dikkers ${ }^{5}$, Ton J. van Essen ${ }^{2}$, Andrea Superti-Furga ${ }^{6}$, Elias Alexandrakis ${ }^{7}$, Cees Vermeer ${ }^{1}$, Leon J. Schurgers ${ }^{1}$, Goos D. Laverman ${ }^{8}$

${ }^{1}$ VitaK and Cardiovascular Research Institute Maastricht (CARIM), Maastricht University, Maastricht, The Netherlands; ${ }^{2}$ Department of Genetics, University Medical Center Groningen, University of Groningen, Groningen, the Netherlands; ${ }^{3}$ Division of Molecular Paediatrics, Centre Hospitalier Universitaire Vaudois, Lausanne, Switzerland; ${ }^{4}$ Department of Radiology, University Medical Center Groningen, University of Groningen, Groningen, the Netherlands; ${ }^{5}$ Department of Otorhinolaryngology, University Medical Center Groningen, University of Groningen, Groningen, the Netherlands; ${ }^{6}$ Centre for Paediatrics and Adolescent Medicine, Freiburg University Hospital, Freiburg, Germany; ${ }^{7}$ Department of Pathology, Klinikum Kassel, Kassel, Germany; ${ }^{8}$ Department of Internal Medicine, Division of Nephrology, University Medical Center Groningen, University of Groningen, Groningen, the Netherlands

* The first two authors have contributed equally to this work. 


\begin{abstract}
Matrix $\mathrm{\gamma}$-carboxyglutamate (Gla)-protein (MGP), a vitamin K-dependent protein, is recognised as a potent local inhibitor of vascular calcification. Studying patients with Keutel syndrome (KS), a rare autosomal recessive disorder resulting from MGP mutations, provides an opportunity to investigate the functions of MGP.

We describe a novel homozygous MGP mutation (c. $61+1 \mathrm{G}>\mathrm{A}$ ) in a newly identified KS patient, of whom the phenotype was carefully characterised. No signs of arterial calcification were found, in contrast to findings in MGP knockout mice that die within two months due to arterial calcification. This patient is the first in whom circulating MGP species were characterised, which revealed a high level of phosphorylated MGP and a low level of carboxylated MGP. Contrary to expectations, vitamin K supplements did not improve the circulating carboxylated MGP levels. In addition, we characterised MGP species in various affected tissues of the first KS patient originally described in 1971. Phosphorylated MGP was also found to be present in this patient.
\end{abstract}

The investigation of the phenotype and MGP species in the circulation and tissues of KS patients contributes to our understanding of MGP functions in various tissues and to further elucidation of the difference in arterial phenotype between MGP-deficient mice and humans. 


\section{Rare mutations in the gene encoding matrix Gla-protein (MGP): \\ a new case of Keutel syndrome serves as a model \\ for exploring MGP functions in humans}

\section{Introduction}

Matrix $\gamma$-carboxyglutamate (Gla)-protein (MGP) is increasingly recognised as a potent inhibitor of arterial calcification with important clinical implications. MGP is present in the vascular wall and in cartilage, where it is synthesised by vascular smooth muscle cells and chondrocytes, respectively. It can undergo two post-translational modifications: $\gamma$ glutamate carboxylation, which is vitamin K-dependent, and serine phosphorylation. Uncarboxylated MGP is invariably found to accumulate in atherosclerotic lesions and areas of arterial calcification in tissue; high circulating levels are found in patients with a high risk of prevalent cardiovascular calcification [1-3]. The two post-translational modifications result in several MGP species (carboxylated, non-carboxylated, phosphorylated, and non-phosphorylated MGP). Carboxylation, which is vitamin K-dependent, enhances the binding of MGP to calcium nuclei in hydroxyapatite $[4,5]$ and is a prerequisite for its inhibition of bone morphogenetic protein-2 (BMP-2) [6-8], an osteogenic growth factor. Although the exact function of phosphorylation is unclear, it may regulate MGP secretion into the extracellular environment [9]. Several enzyme-linked immunoassays (ELISAs) have been developed to measure different MGP species in the circulation.

Genetically determined loss of MGP function may provide important insights into the functions of the protein. MGP knockout mice were born without abnormalities, but developed severe calcification of all elastic and muscular arteries, eventually resulting in rupture of the calcified aorta and subsequent death within two months [10]. Histological examination of MGP knockout mice revealed extensive calcification of the elastic lamellae at the age of two weeks, and the appearance of cartilage nodules in the calcified aorta at the age of one month [11]. They also displayed cartilage calcification at sites of proliferating chondrocytes: the lower end of the trachea, the main bronchi, and the growth plate, which resulted in short stature, osteopenia, and fractures [10].

Abnormal cartilage calcification is the hallmark of Keutel syndrome (KS), an extremely rare autosomal recessive disorder in humans, resulting from mutations in the MGP gene [12]. So far, only 26 patients with KS have been described in the literature [12-28]. In addition to abnormal cartilage calcification, KS is characterised by brachytelephalangism, peripheral pulmonary artery stenosis, hearing loss (sensorineural, mixed, and conductive), and facial abnormalities (i.e. mid-face hypoplasia and a depressed nasal bridge) [26]. Four MGP mutations have been reported thus far, which all predict absent or non-functional MGP $[12,26]$. Remarkably, KS patients do not seem to suffer from extensive arterial calcification. 
We describe a novel MGP mutation, found in a newly identified KS patient. His phenotype was carefully characterised, paying specific attention to signs of vascular calcification. In addition, this is the first patient in whom circulating MGP species have been characterised. These measurements were used to evaluate whether he would benefit from vitamin $\mathrm{K}$ supplements. In addition to these studies, we investigated the presence of MGP and other calcification-inhibitors in tissues from the first KS patient originally described by Keutel et al. [13]. 


\section{Rare mutations in the gene encoding matrix Gla-protein (MGP): \\ a new case of Keutel syndrome serves as a model \\ for exploring MGP functions in humans}

\section{Materials and Methods}

\section{Subjects}

A male patient was referred from the Department of Otorhinolaryngology to our clinical genetics outpatient clinic and subsequently diagnosed with Keutel syndrome. This patient and his first-degree relatives (parents and two siblings) were included in our study. All participants gave their informed consent. To assess the effect of increased vitamin $\mathrm{K}$ intake on his circulating MGP levels, he was prescribed $10 \mathrm{mg}$ vitamin $\mathrm{K}_{1}$ (phylloquinone; Konakion, Roche Nederland, Woerden, the Netherlands) daily for 3 months at the age of 21 years. This is the standard form and dosage of vitamin $\mathrm{K}$ which is prescribed in the Netherlands.

No tissue from our patient was available for immunohistochemical staining. Fortunately, tissues from another male patient diagnosed with Keutel syndrome were available. This was one of the two siblings originally described by Keutel et al. [13]. Meier et al. described the follow-up and post-mortem examination of this patient (their case 1) in 2001 [29]. He died at age 38 years due to right heart failure. He had previously suffered from increasing dyspnoea and coughing, seizures with ischemic cerebral lesions, and had been diagnosed with mediastinal seminoma, for which he had received chemotherapy.

\section{Genetic analysis}

Genomic DNA was isolated from blood samples (QIAamp DNA Blood Mini Kit) obtained from our newly identified patient with KS and his four first-degree relatives. The four coding exons, as well as exon-intron boundaries, of MGP were amplified by polymerase chain reaction (PCR) using the following oligonucleotides: exon1- $F$ (CTCTCAACTGCTCTGGTTC) and exon1-R (AAGTAAGCCAAAGTCAGAGGC), exon2-F (TTTCCTCTTCTTCCATCCCTG) and exon2-R(CCCTCCCTGTTATATATCTTTC), exon3-F (TATTCACGGAAATATTTCCAGC) and exon3-R (CAGATCTGTGATCTACACTG), exon4-F (GTATTTTTCCACTTTATCCTTC) and exon4-R (AAAATCAGGTGCCAGCCTC), and analysed by bidirectional fluorescent direct sequencing. Results were confirmed in a second amplification product.

A skin biopsy of the patient was performed to obtain fibroblasts, of which mRNA was extracted. The effect of the identified MGP mutation on mRNA splicing has been elucidated by performing RT-PCR on the obtained mRNA. The entire MGP coding sequence was amplified by RT-PCR and analyzed by bidirectional fluorescent direct sequencing. 


\section{Biochemical measurements}

Blood samples were collected from our patient and, for comparison, from his first-degree relatives. For circulating MGP and osteocalcin measurements, blood was collected by venipuncture in serum (10 ml; BD Vacutainer systems, Plymouth, UK) and in sodium citrate tubes (10 ml; BD Vacutainer systems, Plymouth, UK) and stored for 20 minutes at room temperature before centrifugation $(15 \mathrm{~min}, 1580 \mathrm{~g}$ ). All data are means of duplicate measurements, unless stated otherwise.

Circulating MGP levels were quantified with four different MGP ELISA. Circulating nonphosphorylated MGP was measured with a kit from Biomedica (Vienna, Austria), in which the monoclonal antibody against the human non-phosphorylated MGP residues 3-15 is coated to the microtitre plate [30]. Circulating phosphorylated MGP was measured with a competitive ELISA, using a monoclonal antibody raised against the phosphorylated MGP residues 3-15 (VitaK BV, Maastricht, the Netherlands). The antibody was selected for its specificity towards phosphorylated MGP using standard techniques, as described for the other MGP antibodies [1]. For the phosphorylated MGP ELISA, the antibody was coupled to the microtitre plate. Serum sample or standard was supplemented with tracer (biotinylated phosphorylated MGP) and transferred to the microtitre plate and incubated overnight at $4^{\circ} \mathrm{C}$. MGP concentration was calculated with the aid of a calibration curve of synthetic phosphorylated MGP. Biotinylated, synthetic phosphorylated MGP was made by Thinkpeptides (Prolmmune, Oxford, UK).

Circulating uncarboxylated and carboxylated MGP were quantified with two sandwich ELISA (VitaK BV). In these assays, the antibody against non-phosphorylated MGP residues 3-15 served as a detecting antibody. The biotinylated antibodies against the uncarboxylated MGP residues 35-49 and the carboxylated MGP residues 35-54 served as capture antibodies, respectively [31]. These assays seem to be particularly suited to assess the effect of changes in vitamin $\mathrm{K}$ status on MGP levels.

The carboxylation status of circulating osteocalcin (OC), a vitamin K-dependent protein present in bone, was assessed by measurements of uncarboxylated (ucOC) and carboxylated (COC) osteocalcin using dual-antibody OC ELISAs (Takara, Shiga, Japan). The ratio of $\mathrm{ucOC}$ and $\mathrm{COC}(\mathrm{ucOC} / \mathrm{cOC}$ ) was calculated from these measurements. A decrease in $\mathrm{ucOC} / \mathrm{cOC}$ over time indicates an improved vitamin $\mathrm{K}$ status [32]. 


\section{Rare mutations in the gene encoding matrix Gla-protein (MGP): \\ a new case of Keutel syndrome serves as a model for exploring MGP functions in humans}

Immunohistochemistry

Immunohistochemistry for Keutel's original first male patient was performed on sections of lung (from trachea to bronchi of the third generation), aorta, skin, and heart tissue embedded in paraffin. All sections were stained for hematoxylin and eosin (HE). Von Kossa and Elastica von Gieson staining were performed to visualise calcification and elastic fibres, respectively. Immunostaining for MGP was performed as described previously [1], with the same monoclonal MGP antibodies as used in the MGP ELISA directed against phosphorylated MGP, non-phosphorylated MGP, carboxylated MGP, and noncarboxylated MGP. Sections were also stained with rabbit anti-human fetuin-A (BioVendor, Heidelberg, Germany) and rabbit anti-human osteopontin (Abcam, Cambridge, UK). For MGP, staining was performed using biotinylated sheep anti-mouse IgG (60 minutes at room temperature; Dako, Golstrup, Denmark) as a secondary antibody, followed by incubation with avidin-linked alkaline phosphatase complex (30 minutes at room temperature; Dako). Staining was then performed using the Vector Red Alkaline Phosphatase Substrate kit I (Vector Laboratories, Burlingame, CA, USA), yielding a red colour. For fetuin-A and osteopontin, biotinylated goat anti-rabbit IgG was used as a secondary antibody, followed by incubation with peroxidase-labelled streptavidin (LSAB2 System-HRP, Dako), according to the manufacturers' instructions. Staining was then performed by the Vector NovaRED substrate kit (Vector Laboratories), yielding a brown colour. Sections were counterstained with hematoxylin. Controls for the immunoreactions were performed by omitting the primary antibody. 


\section{Results}

\section{Phenotypic characterisation}

A 16-year old male was referred to the Department of Otorhinolaryngology, University Medical Center Groningen, for evaluation of exertional dyspnoea. His medical history included mild congenital bilateral peripheral pulmonary stenosis and respiratory problems, refractive to the use of bronchodilators. Psychomotor development was normal. He is the second child of consanguineous Turkish parents, living in the Netherlands. He has two sisters and the younger one has a history of childhood epilepsy. All four first-degree relatives were apparently unaffected.

Physical examination of the patient revealed a slight inspiratory stridor on auscultation. There were no obvious dysmorphic facial features (Figure 1). However, his auricles were strikingly stiff, his chest was asymmetric, and his hands showed shortening of all distal phalanges. His height was -1 SD for age (with target height plus 1 SD) according to Turkish growth charts.

Lung function tests revealed obstructive upper airway disease. Laryngotracheoscopy showed a short and posteriorly tilted epiglottis, leading to difficult intubation. The vocal folds were remarkably short with a large posterior chink. The cricoid cartilage was stenosed (Figure 2A), providing the explanation for his exertional dyspnoea. The trachea showed an irregular appearance (Figure $2 \mathrm{~B}$ ). A CT scan revealed extensive cartilage calcification of his larynx, trachea, main bronchi, costochondral junctions, and auricles. The severity of the stenosis of the cricoid cartilage was clearly shown by three-dimensional CT scan reconstructions (Figure 3). Hearing thresholds were $10 \mathrm{~dB}$ (normal) for the right ear, with a $20 \mathrm{~dB}$ conductive hearing loss for the high tones in the left ear. An electrocardiogram showed no abnormalities. An echocardiogram showed normal dimensions and no functional abnormalities except for a small increase in the flow of the left pulmonary artery. Ophthalmological evaluation did not reveal any abnormalities and he did not have any skin abnormalities. No abnormalities were found with routine blood haematology and chemistry, including calcium, phosphate, liver function tests, and clotting tests. Overall, these features were best compatible with a diagnosis of Keutel syndrome (Table 1) and mutation analysis of the MGP gene was performed to confirm this diagnosis. 
Rare mutations in the gene encoding matrix Gla-protein (MGP):

a new case of Keutel syndrome serves as a model for exploring MGP functions in humans

Figure 1: Frontal and lateral view of our patient

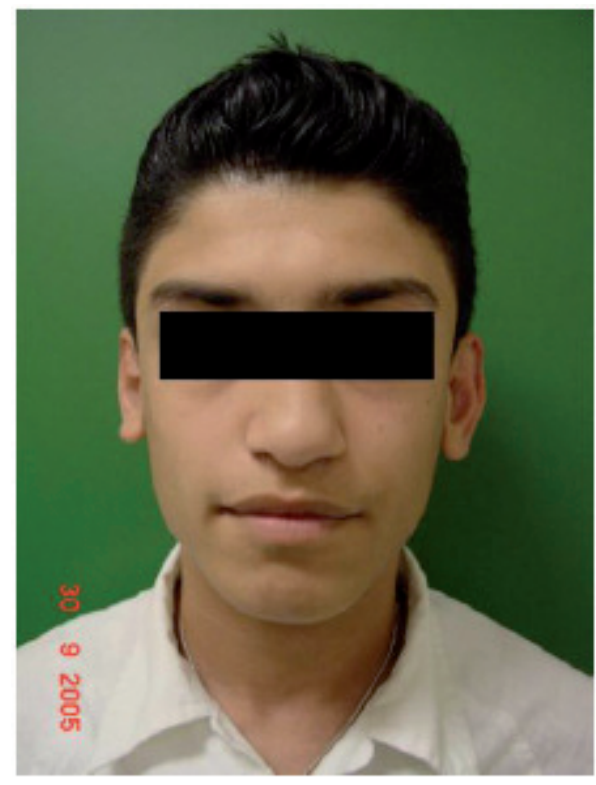

A

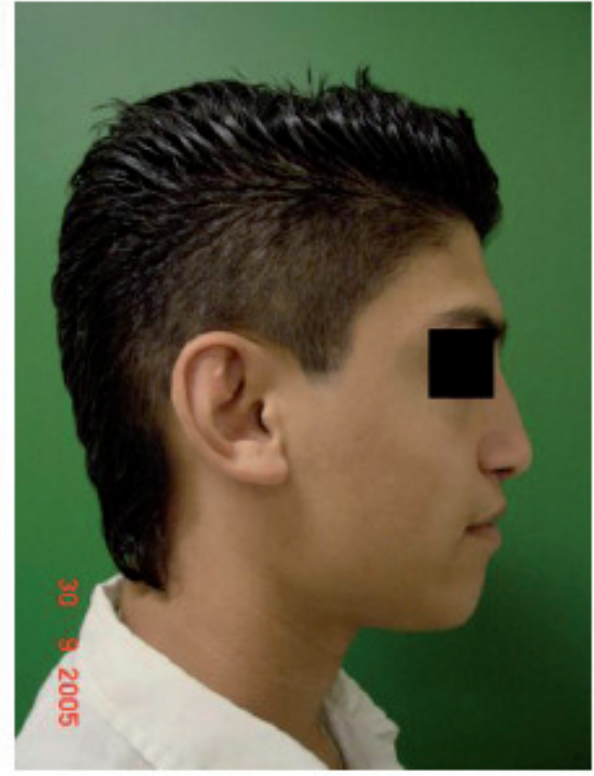

B

Frontal (A) and lateral (B) view of patient. Note the calcification present in the patient's auricle.

Figure 2: Laryngotracheoscopy
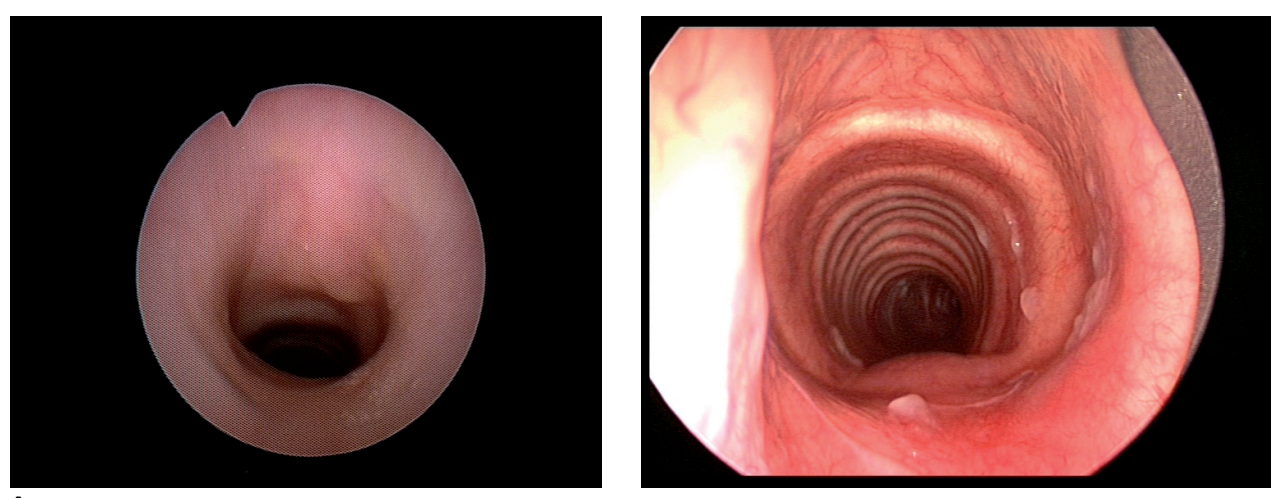

A

a 


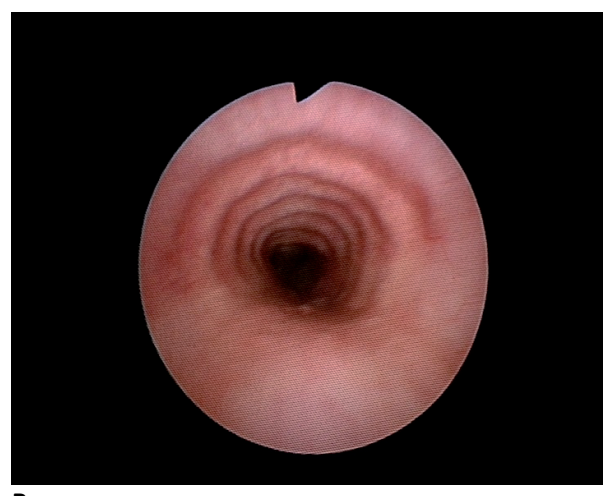

B

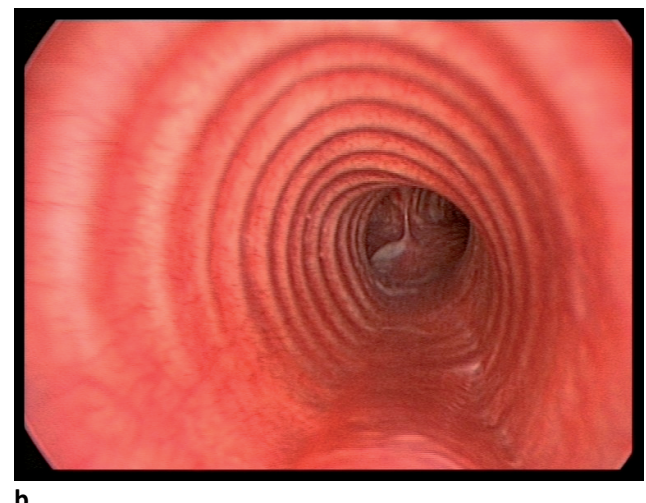

Laryngotracheoscopy images illustrating the stenosed cricoid cartilage (A) and irregular appearance of the trachea of the patient (B), with images of the normal configuration for comparison (a,b). (A) Patient's cricoids with irregular stenosed appearance. No visible tracheal rings due to stenosis. (a) Far left the left true vocal cord can be seen, and in the center the almost circular appearance of cricoids. Several patches of mucus are present. The trachea is visible distally. (B) Patient's irregular narrowed lumen of the trachea with tracheal rings shining through mucosa. (b) Regular smooth tracheal rings. Note: Do not compare picture size, but configuration.

Figure 3: Three-dimensional CT scan reconstructions

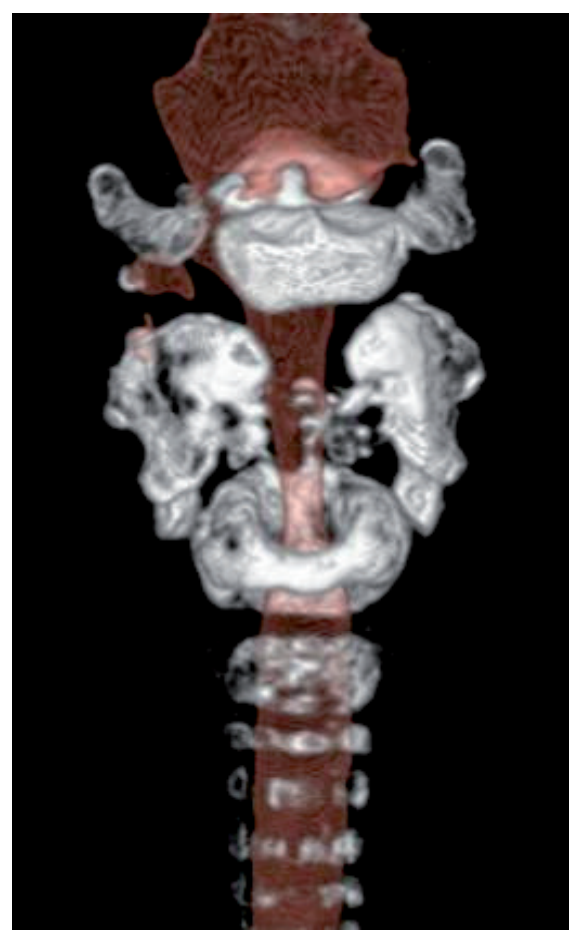

A

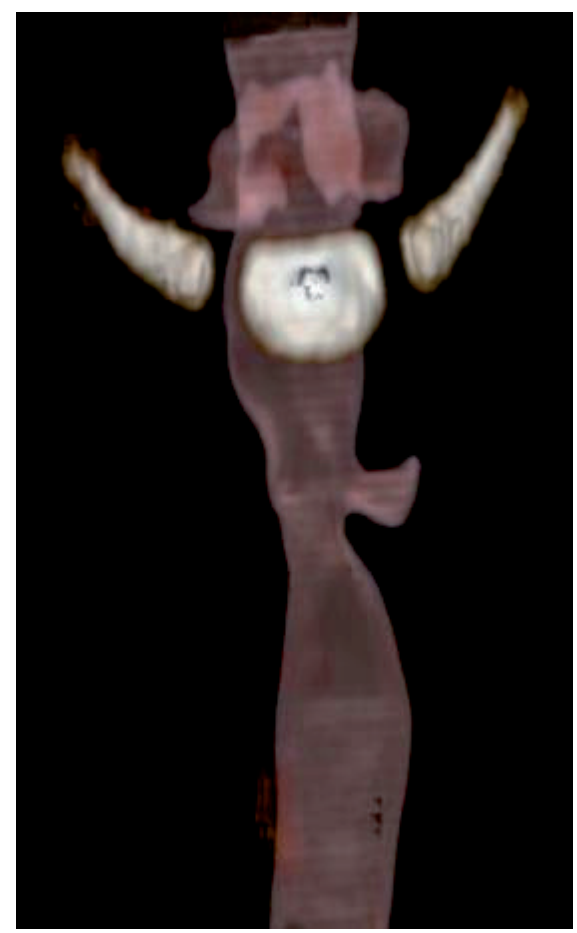

a 


\section{Rare mutations in the gene encoding matrix Gla-protein (MGP): \\ a new case of Keutel syndrome serves as a model for exploring MGP functions in humans}

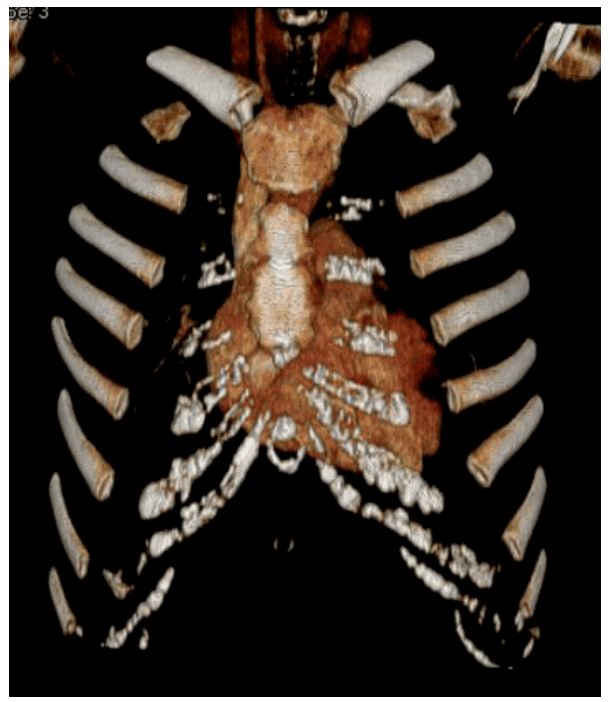

B

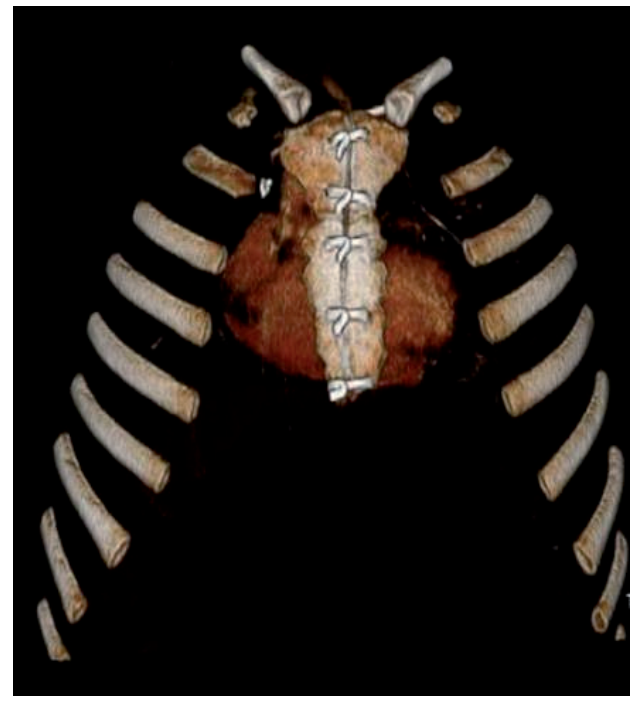

b

Three-dimensional CT scan reconstructions illustrating the severity of the patient's cricoid cartilage stenosis (A), and calcifications of his rib cartilage (B), with images of the normal configuration for comparison $(a, b)$.

(A) CT neck, volume dataset, virtual rendering mode, anterior-posterior view: pronounced ossification of hyoid and extensive calcification of cricoids, thyroid, and tracheal ring cartilage visible in white. Air band of hypopharynx and proximal trachea visible in red. (a) Patient of the same age with small left lateral laryngocele. Normal ossification of hyoid. No cartilage calcifications visible.

(B) CT thorax, volume dataset virtual rendering mode anterior-posterior view: Calcifications of bilateral rib arch cartilage (white) visible medial to the regularly ossified ventral ribs. (b) Patient of the same age (status after sternotomy). No rib cartilage calcifications visible.

Note the extensive calcification of the cricoid, thyroid, hyoid and tracheal rings. 
Table 1: Clinical features of Keutel syndrome

\begin{tabular}{|c|c|c|}
\hline Clinical features & Our patient & Literature $(n=26)$ \\
\hline Age at diagnosis (years [mean $\pm S D]$ ) & 16 & $8.3 \pm 4.8$ \\
\hline Gender & M & $11 \mathrm{M}, 15 \mathrm{~F}$ \\
\hline Consanguinity & + & $19 / 21$ \\
\hline \multicolumn{3}{|l|}{ Cartilage calcification } \\
\hline Nose (septum, ala nasi) & - & $8 / 8$ \\
\hline Ear (outer and middle) & + & $12 / 12$ \\
\hline Larynx & + & $13 / 13$ \\
\hline Trachea and/or bronchi & + & $20 / 20$ \\
\hline Ribs & + & $9 / 9$ \\
\hline Epiphyseal stippling & - & $3 / 5$ \\
\hline \multicolumn{3}{|l|}{ Characteristic facial features } \\
\hline Midface hypoplasia & - & $14 / 14$ \\
\hline Broad, depressed nasal bridge & - & $21 / 21$ \\
\hline Brachytelephalangism & + & $25 / 25$ \\
\hline Respiratory problems & + & $14 / 18$ \\
\hline Recurrent otitis media / sinusitis & - & $9 / 13$ \\
\hline Hearing loss & right -, left + & $12 / 17$ \\
\hline Peripheral pulmonary stenosis & + & $14 / 17$ \\
\hline Heart defects ${ }^{A}$ & - & $3 / 15$ \\
\hline Cerebral defects $^{\mathrm{B}}$ & - & $4 / 12$ \\
\hline Seizures & - & $4 / 6$ \\
\hline Developmental delay & - & $5 / 15$ \\
\hline Mild short stature (length $\leq 10^{\text {th }}$ percentile) & - & $10 / 21$ \\
\hline
\end{tabular}

Summary of the clinical features of our patient and the patients with Keutel syndrome described in the literature

A Reported heart defects are ventricular septal defect $(n=1)$, patent ductus arteriosus and foramen ovale $(n=1)$, and thick mitral valve leaflets $(n=1)$.

${ }^{B}$ Reported cerebral defects are multiple calcifications $(n=2)$, leukodystrophy (with bilateral periventricular and subcortical with matter changes on brain MRI after 3 years; $n=1$ ), mild atrophy of the cerebral hemisphere (secondary to meningitis; $n=1$ ), and encephalomalacia $(n=1)$.

Vascular evaluation was performed when the patient was 21 years old, to investigate whether any arterial calcification was present. He had no complaints consistent with cardiovascular disease. His blood pressure was $122 / 78 \mathrm{~mm} \mathrm{Hg}$, the ankle-brachial index was $108 \%$ on the right, $112 \%$ on the left side, and after exercise $82 \%$ and $79 \%$, respectively (values above $140 \%$ may indicate arterial calcification [33]). Carotid intima-media thickness of the right and left common carotid arteries were both $0.45 \mathrm{~mm}$; right and left internal carotid arteries $0.45 \mathrm{~mm}$ and $0.40 \mathrm{~mm}$, respectively; bulbus right and left $0.5 \mathrm{~mm}$ and $0.4 \mathrm{~mm}$, respectively (ultrasound upper limit of normal range for adolescents [34]). No arterial calcifications were seen on a chest X-ray of the upper abdomen (hepatic, lienal, renal arteries). A cranial CT scan did not reveal any arterial calcifications of intracranial arteries of the circle of Willis. Multi-slice CT (MSCT) scanning of the coronary arteries revealed no calcification (calcium score 0 ). 


\section{Rare mutations in the gene encoding matrix Gla-protein (MGP): \\ a new case of Keutel syndrome serves as a model for exploring MGP functions in humans}

\section{MGP mutation}

The clinical diagnosis was confirmed by the identification of a novel, homozygous MGP mutation in this patient: c.61+1G>A, indicating nucleotide substitution of guanine (G) by adenine (A) in cDNA of the first nucleotide in the introns after base 61 (donor splice site)ENTER weg(Figure 4). The effect of this mutation on mRNA splicing has been elucidated by performing RT-PCR. This identified mutation eliminates the consensus donor splice site at the end of exon 1 (TG-gt is replaced by TG-at). Instead of this mutated splice site, a new donor splice site is used, 19 bases upstream of the usual one. Thus the mutation results in the deletion of 19 bases in the cDNA, which gives rises to a frameshift. $A$ novel stop codon in the 3'untranslated sequence of the MGP gene is used, 49 bases downstream of the normal stop codon. The new predicted protein is composed of 113 amino-acids (instead of 103) and only 14 amino-acids of the original sequence are conserved in the sequence upstream of the deletion. Both parents and the older sister of the patient are heterozygous carriers of the mutation. His younger sister does not carry the mutation.

Figure 4: $M G P$ gene and identified mutations

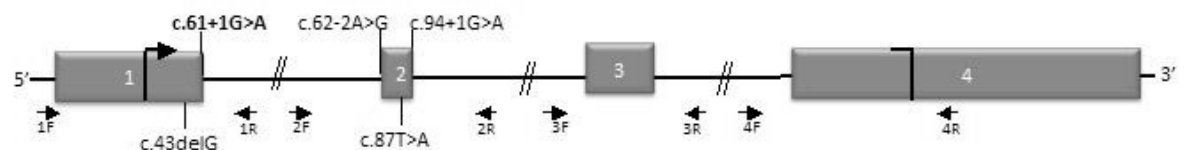

The MGP gene in which exons are shown as boxes and introns as lines (not to scale). Oligonucleotides used for PCR and sequencing are noted as arrows. The start codon is indicated by an arrow and the stop codon by a line, at the top of the exons. Nucleotide 1 has been counted as the transcription initiation site. Mutations previously reported are indicated, and the mutation reported here is indicated in bold. Mutations affecting splice sites are indicated above the gene, other mutations are indicated below.

Circulating MGP species were measured in our patient's father (parent 1, age 43), mother (parent 2, age 42), our patient (age 19), his older sibling (sibling 1, age 20), and in his younger sibling (sibling 2, age 15). Non-phosphorylated MGP and phosphorylated MGP (A) as well as uncarboxylated and carboxylated MGP (B) could be detected in blood samples from the patient and his family members. For reference, the normal range for each measured MGP species is given in brackets. The normal range, defined as the mean $\pm 2 \times$ standard deviation (SD), was based on MGP measurements in 50 healthy subjects. The mean \pm SD age of this reference population ( $28 \pm 7$ years) was comparable with that of the patient and family members ( $28 \pm 14$ years). 


\section{Circulating MGP}

Four different assays were used to characterise circulating MGP. The circulating nonphosphorylated and phosphorylated MGP levels (Figure 5A) of the patient and his firstdegree relatives were within the normal range. He had the lowest concentration of nonphosphorylated MGP in comparison with his relatives. In contrast, he had the highest concentration of phosphorylated MGP, which was 1.5-2 times as high as those of his relatives. The circulating uncarboxylated and carboxylated MGP levels (Figure 5B) were low to very low in all family members, including our patient.

Since MGP carboxylation depends on the presence of vitamin K, we aimed to investigate whether increased intake of vitamin $\mathrm{K}$ would influence his MGP levels. In theory, increased MGP carboxylation would enhance its calcification-inhibitory activity, which would have a beneficial effect in preventing the development of arterial calcification. The measurements of uncarboxylated and carboxylated MGP are suited for detecting the effect of changes in vitamin K status on MGP levels. Uncarboxylated MGP, in particular, is sensitive for changes in vitamin $\mathrm{K}$ status and decreases significantly within 12 weeks upon vitamin $\mathrm{K}$ supplementation in healthy subjects [31]. The patient received $10 \mathrm{mg}$ vitamin $\mathrm{K}_{1}$ daily during 3 months. The decrease in the ucOC/cOC ratio from 0.70 at baseline to 0.08 after 3 months of supplements confirmed that his systemic vitamin $\mathrm{K}$ status had improved. The results of MGP measurements are shown in Figure 6. The uncarboxylated MGP levels remained unchanged; there was a decrease in carboxylated MGP levels after 3 months. This small decrease is within the intra-individual variation, and was not considered to be an effect of increased vitamin $\mathrm{K}$ intake. It was therefore concluded that the increased intake of vitamin $\mathrm{K}_{1}$ during 3 months had not affected circulating MGP levels in our patient. 


\section{Rare mutations in the gene encoding matrix Gla-protein (MGP): \\ a new case of Keutel syndrome serves as a model for exploring MGP functions in humans}

Figure 5. Circulating MGP measurements

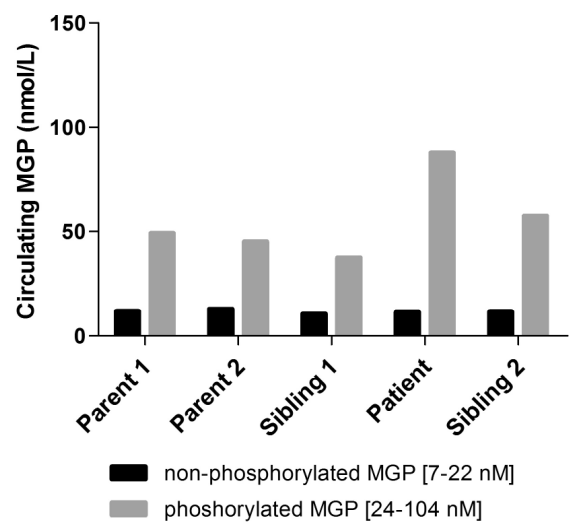

A

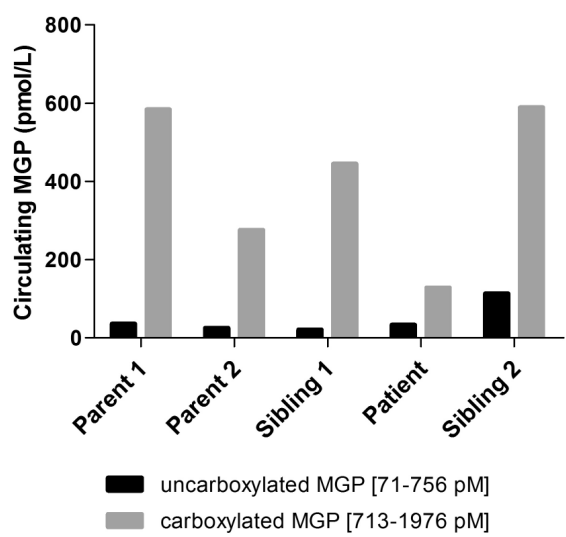

B

Circulating MGP species were measured in our patient's father (parent 1, age 43), mother (parent 2, age 42), our patient (age 19), his older sibling (sibling 1, age 20), and in his younger sibling (sibling 2, age 15). Nonphosphorylated MGP and phosphorylated MGP (A) as well as uncarboxylated and carboxylated MGP (B) could be detected in blood samples from the patient and his family members. For reference, the normal range for each measured MGP species is given in brackets. The normal range, defined as the mean $\pm 2 \times$ standard deviation (SD), was based on MGP measurements in 50 healthy subjects. The mean \pm SD age of this reference population ( $28 \pm 7$ years) was comparable with that of the patient and family members ( $28 \pm 14$ years). 
Figure 6. Effect of vitamin K supplementation on MGP levels

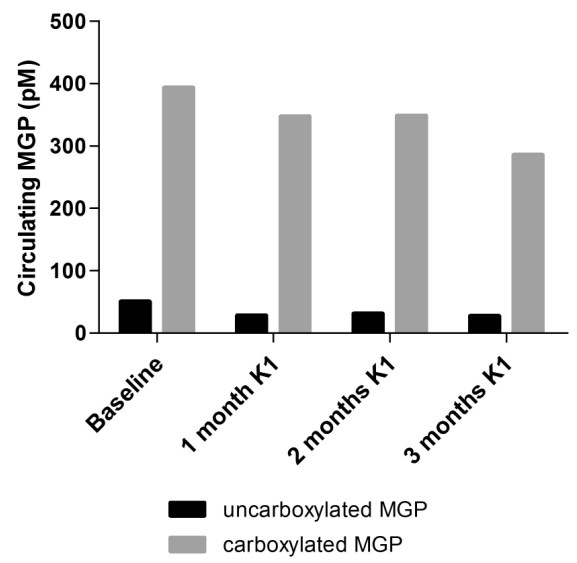

Circulating uncarboxylated and carboxylated MGP levels of the patient are shown at baseline and after 3 months of vitamin $\mathrm{K}_{1}$ supplementation ( $10 \mathrm{mg}$ daily). The uncarboxylated MGP level remained unchanged. Although there was a very small decrease in the carboxylated MGP level, this was not considered to be clinically significant. 


\section{Rare mutations in the gene encoding matrix Gla-protein (MGP): \\ a new case of Keutel syndrome serves as a model for exploring MGP functions in humans}

\section{MGP in tissue}

$\mathrm{HE}$ and Von Kossa staining of lung tissue from the first male patient described by Keutel et al. revealed concentric arterial calcification in the region of the internal elastic lamina of the small- and medium-sized pulmonary arteries, which was often accompanied by intimal hypertrophy (Figure 7A). This type of calcification was also seen in the coronary arteries and skin arteries. In the lung tissue, calcifications were also seen in the connective tissue surrounding the bronchi and vasculature. The presence of extra-vascular calcifications was also clearly demonstrated by Von Kossa staining of the skin, revealing ossifications and elastic fibre calcifications in the dermis (Figure 7A). Staining for MGP was found to be positive in the arteries of the different tissues, with the predominant MGP species being phosphorylated MGP (Figure 7B). Staining for fetuin-A was positive in the vasculature (not shown), as was osteopontin staining. Positive osteopontin staining was also seen in the aortic media, in which Von Kossa staining revealed minor calcifications along the elastic lamellae (Figure 7B).

Figure 7: Immunohistochemical stainings. (See next page.)

7A I, II: Hematoxylin and eosin (HE) and Von Kossa staining of lung tissue

7A III, IV: HE and Von Kossa staining of skin tissue

7B I, II: Phosphorylated MGP stainings of lung tissue

7B III, IV: Von Kossa and osteopontin staining of the aorta

Original magnifications x100 (AI, AII, AIV, BI) and x200 (AIII, BII-IV). HE staining of lung tissue obtained at postmortem examination of the male patient originally described by Keutel et al. revealed extensive distortion of normal anatomy, indicating arterial calcification (A I; arrows). Specks of calcifications are also seen in the connective tissue surrounding the bronchi and arteries (A II; black, arrow). The presence of extra-vascular calcifications was also clearly demonstrated in the dermis of the right thigh, revealing ectopic calcifications (A III and IV; arrow) and elastic fibre calcifications (A IV; black, dotted arrow). The presence of MGP in the tissue was clearly demonstrated by staining of phosphorylated MGP in the media (M) of a pulmonary artery, which appeared to colocalize with vascular smooth muscle cells (B I; red, arrow). A band of tissue with the morphological appearance of cartilage $(C)$ was present at the luminal side of an extensively calcified artery, with the chondrocyte-like cells positively stained for phosphorylated MGP (B II; red, arrow). Von Kossa staining of the aorta revealed calcium deposition along the elastic fibres in the aortic media (B III; black, arrows). Osteopontin staining was positive in the aortic media (B IV; brown, arrow). M: Media; L: Lumen; C: Cartilage. 
Figure 7: Immunohistochemical stainings

$\mathrm{HE}$

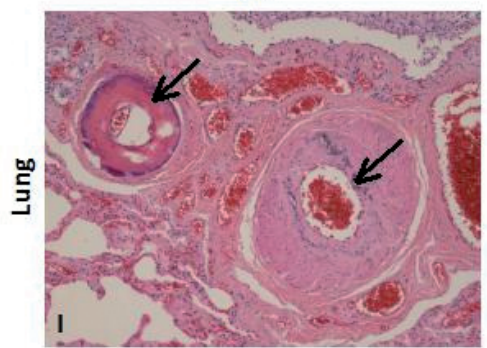

HE

A

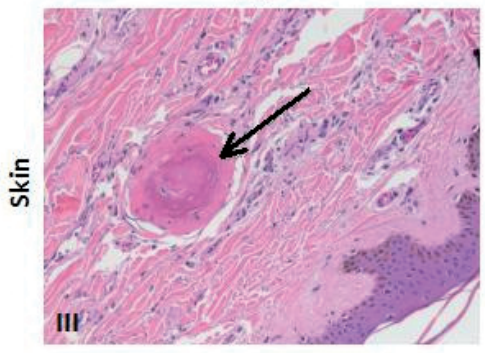

Von Kossa

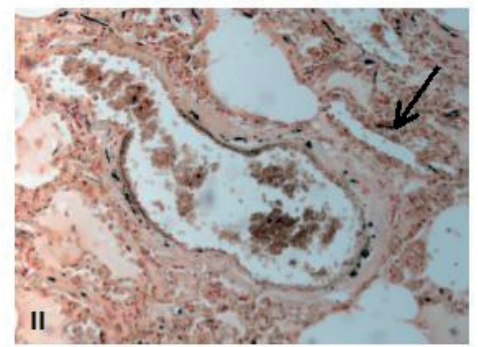

Von Kossa

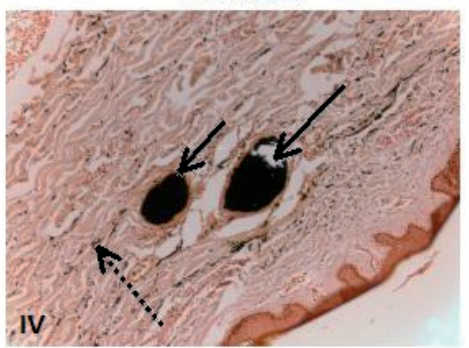

Phosphorylated MGP

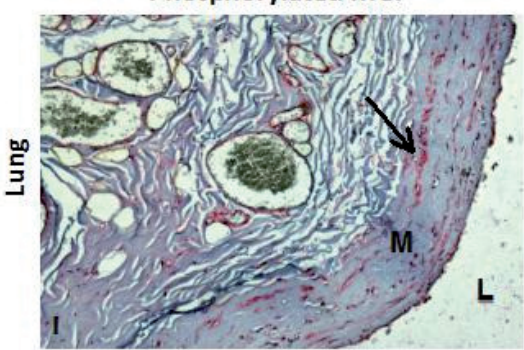

Von Kossa

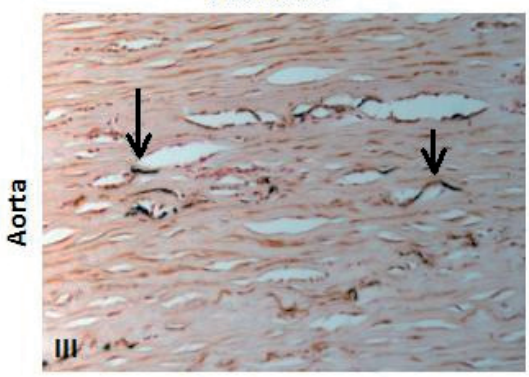

Phosphorylated MGP

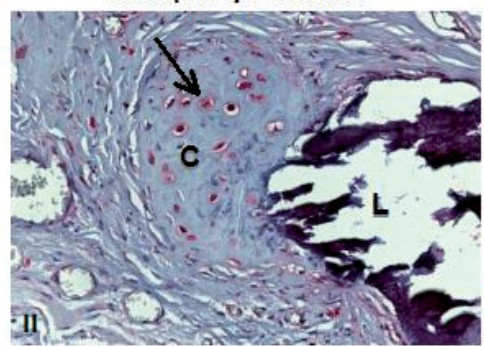

Osteopontin

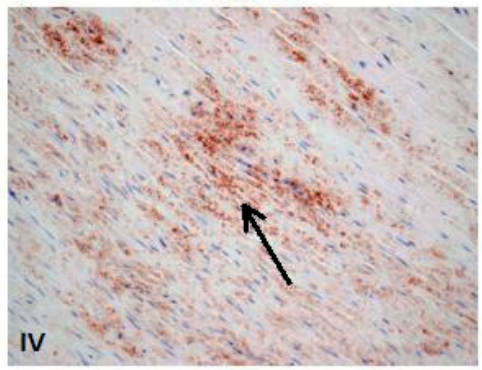




\section{Rare mutations in the gene encoding matrix Gla-protein (MGP): \\ a new case of Keutel syndrome serves as a model \\ for exploring MGP functions in humans}

\section{Discussion}

This study was performed in two patients with Keutel syndrome (KS), a rare autosomal recessive disorder resulting from mutations in the gene encoding the protein MGP which inhibits calcification and is vitamin K-dependentK. First we describe a novel homozygous MGP mutation in a newly identified KS patient. Second, this is the first report on circulating MGP species in the presence of a functional mutation and it demonstrates high levels of phosphorylated MGP and low levels of carboxylated MGP. Third, we found that vitamin $\mathrm{K}$ supplements did not improve the patient's MGP carboxylation. Finally, we were able to demonstrate the presence of phosphorylated MGP in the vasculature, obtained at postmortem examination, of the first KS patient originally described by Keutel et al. [13].

Our patient presented with exertional dyspnoea refractive to bronchodilatory drugs. Laryngotracheoscopy revealed cricoid stenosis and an abnormal trachea, and CT scanning showed abnormal cartilage calcification. Other features, including brachytelephalangism and peripheral pulmonary artery stenoses, were consistent with the diagnosis of KS. Mutation analysis revealed a novel MGP mutation (c.61+1G>A). This is the fifth MGP mutation identified so far. This particular mutation results in the loss of the consensus donor splice site at the exon1-intron1 junction. The previously identified MGP mutations were two splice site mutations and two introducing a stop codon $[12,26]$. Although all these mutations are expected to coincide with absent or non-functional MGP, circulating MGP species have not been previously characterised in KS patients.

In our patient, measurements of circulating MGP revealed a high level of phosphorylated MGP and a low level of carboxylated MGP compared to both his heterozygous first-degree relatives and to the sibling without the MGP mutation. The presence of different MGP species (nonphosphorylated, phosphorylated, non-carboxylated, carboxylated MGP) originates from two post-translational modifications that may occur: vitamin K-dependent carboxylation and serine phosphorylation. Phosphorylation of extracellular matrix proteins is thought to be important for control of biomineralization [35]. This has been extensively studied for osteopontin (OPN), a phosphorylated glycoprotein that inhibits soft tissue calcification. Non-phosphorylated OPN greatly reduces its ability to inhibit hydroxyapatite formation as well as smooth muscle cell calcification [36-38]. It has been postulated that phosphorylation results in a protein conformation change increasing the protein's affinity for calcium crystals thereby limiting their growth and proliferation [35]. Moreover, it may facilitate the recruitment and activation of macrophages that remove pathologic calcium deposits [39]. For MGP, a (additional) function for phosphorylation may be the regulation of MGP secretion from the cell [9]. Moreover, it has been shown in experiments with vascular smooth muscle cells that phosphorylated MGP residues (without carboxylated MGP residues) may also partly inhibit calcification [40]. 
The phosphoserine domain, the Gla domain, and the C-terminus seem to be important for normal MGP function. The high level of phosphorylated MGP in our patient may be explained by a relatively high synthesis of MGP, possibly induced by the high calcification load. The carboxylation of MGP is a prerequisite for its inhibition of BMP-2, and the low circulation levels of carboxylated MGP indicate that this mechanism of MGP action is likely to be impaired.

MGP acts as a local inhibitor of calcification, which has been illustrated by MGP knockout mice, of which the phenotype can be rescued if MGP expression is selectively restored locally in the vascular smooth muscle cell [41]. However, increasing the circulating MGP level did not rescue the calcifying phenotype [41]. The finding of MGP in the circulation of our patient is therefore not clear proof that MGP still has calcification-inhibitory in the patient. However, it does confirm that MGP is synthesized in the tissues of our patient. Of note, circulating MGP species may reflect various aspects of calcification inhibition by MGP in the tissue.

Vitamin $\mathrm{K}$ deficiency, either due to nutritional deficit or the use of vitamin $\mathrm{K}$ antagonists (e.g. coumarin-derivatives), will result in undercarboxylation of MGP and thus impair its biological function [42]. In contrast, increased availability of vitamin K for MGP in tissue may enhance its calcification-inhibiting activity and possibly reduce the development or progression of calcification in cartilage, the arterial wall, or other soft tissues. We were able to measure circulating uncarboxylated MGP levels in our patient, which have previously been found to decrease with vitamin $\mathrm{K}$ supplements given to healthy subjects. We therefore aimed to assess whether vitamin $\mathrm{K}$ would also affect MGP levels in our patient. If so, this would be a first indication that the patient might benefit from vitamin $\mathrm{K}$ supplements and further exploration of the effect on clinical symptoms would be justified. However, no clinically relevant changes in either uncarboxylated of carboxylated MGP levels were seen after 3 months of vitamin $K_{1}$ supplements. The question arises as to why a beneficial effect from vitamin $\mathrm{K}$ supplements was lacking. We can speculate that carboxylation of MGP was not possible due to the mutation, since only the first 14 aminoacids of the mutated protein are comparable with those of the mature MGP protein. Alternatively, since the levels of uncarboxylated and carboxylated MGP were very low in our patient, a modest effect due to vitamin $\mathrm{K}$ supplements might not have been detected. Since the increased availability of vitamin K did not measurably affect MGP, it is unlikely that vitamin $\mathrm{K}$ supplements would improve the clinical symptoms or the long-term outcome in this particular KS patient. 


\section{Rare mutations in the gene encoding matrix Gla-protein (MGP): \\ a new case of Keutel syndrome serves as a model for exploring MGP functions in humans}

Additional phenotypic characterisation of our patient included a thorough vascular evaluation, which revealed no signs of arterial calcification. In contrast to the MGP knockout mice, which displayed severe arterial calcifications soon after birth, no clinical signs of vascular calcification have been reported in most KS patients. In 2001, Meier et al. reported on the follow-up of the siblings originally described by Keutel et al. [29]. The youngest sibling died at age 38 years from right heart failure, and a post mortem revealed concentric calcification in several vascular beds, including the coronary, hepatic, renal, meningeal, and cerebral arteries. This is the only KS patient in which extensive arterial calcification has been described. Tissues were available from this patient for immunohistochemical staining, although none were available from our own patient. We were able to confirm Meier et al.'s finding of concentric arterial calcification of several vascular beds. We also demonstrated the presence of MGP, predominantly in its phosphorylated form, in the vasculature of this patient. The finding of elastic fibre calcification in the dermis is in accordance with a report from Nanda et al., who demonstrated fragmentation of mid-dermal elastic fibres in biopsies of normal-looking skin from two patients with KS [43]. These results suggest a possible role for MGP in preventing elastic fibre calcification in the skin.

The difference in the arterial phenotype between the MGP knockout mice model and the human KS is intriguing. It could be argued that the apparent absence of arterial calcifications in our patient is due to an insufficient sensitivity of the MSCT scanning to detect early calcifications. It has been shown in vascular tissue from young dialysis patients that apoptosis and damage of vascular smooth muscle cells, accompanied by increased tissue levels of uncarboxylated MGP, precede clinically overt calcification and even positive Von Kossa tissue staining for calcification [44]. Irrespective of this, the KS cases described so far may represent mutations accompanied with some residual calcification-inhibiting activity of MGP, which might be sufficient to prevent the development of arterial calcification. In both the patients presented here we found strong indications of the presence of phosphorylated MGP. Even if this is not the entire mature MGP protein, but fragments of MGP containing phosphoserine residues, binding of calcium crystals by MGP might still be possible.

In the MGP knockout mice, Speer et al. demonstrated that OPN was upregulated and accumulated in the calcified arterial wall [45]. In addition, mice deficient in both MGP and OPN showed twice as much calcification at the age of two weeks compared to MGP knockout mice, and they died approximately two weeks earlier due to vascular rupture [45]. It may therefore be hypothesised that other calcification inhibitors are upregulated in our patient that might prevent the development of arterial calcification. 
Our study is the first to confirm Kaartinen et al.'s hypothesis that KS patients lacking functional MGP have arteries containing mineral-inhibiting OPN, possibly allowing for the extended survival of KS patients compared to MGP knockout mice [46]. Patients with KS may not develop overt arterial calcification unless other risk factors are present: either the loss of another inhibitor or the presence of a promoter of arterial calcification. These initiators might be absent in the relatively young KS patients described in the literature so far (mean age 8.3 years), which is supported by the absence of laboratory abnormalities in 19 patients. In contrast, several potential triggers were present in the only KS patient with extensive arterial calcifications described so far (chronic bronchitis, chemotherapy for recurrent malignancy) [29]. This patient was also older at the time of post-mortem examination than the described patients thus far (38 years).

In conclusion, we characterised the phenotype and circulating MGP species of a patient with KS, in whom we had identified a novel MGP mutation. In addition, we characterised MGP species in the tissues of the first KS patient originally described by Keutel et al.. Phosphorylated MGP was found to be present in both patients, indicating that some residual MGP activity might be present. Obviously, further investigation of the function of MGP phosphorylation is needed. Vitamin K supplements did not affect MGP activity in our patient, but might have a beneficial effect for patients in whom carboxylation of MGP is possible. The investigation of KS patients' phenotype and their circulating MGP species contributes to the understanding of MGP functions in various tissues, and helps to elucidate the difference in arterial phenotype between MGP-deficient mice and humans. 


\section{Rare mutations in the gene encoding matrix Gla-protein (MGP): \\ a new case of Keutel syndrome serves as a model \\ for exploring MGP functions in humans}

\section{Acknowledgements}

We thank the patient and his family members for participating in this study. We thank Elke Magdeleyns and Marjolein Herfs (research technicians of VitaK, Maastricht University) for excellent technical assistance in MGP measurements and immunohistochemical stainings. We thank Carole Chiesa Buzzi for excellent technical assistance in RT-PCR analysis. We thank Jackie Senior (science editor of the Department of Genetics, University Medical Center, Groningen University of Groningen) for providing editorial assistance to the authors during preparation of this manuscript.

\section{References}

1. Schurgers, L.J., et al., Novel conformation-specific antibodies against matrix gamma-carboxyglutamic acid (Gla) protein: undercarboxylated matrix Gla protein as marker for vascular calcification. Arterioscler Thromb Vasc Biol, 2005. 25(8): p. 1629-33.

2. Cranenburg, E.C., et al., The circulating inactive form of Matrix Gla Protein (UcMGP) as a biomarker for cardiovascular calcification J Vasc Res 2008. 45: p. 427-36.

3. Cranenburg, E.C.M., et al., Uncarboxylated matrix Gla protein (ucMGP) is associated with coronary artery calcification in haemodialysis patients. Thromb Haemost, 2009. 101(2): p. 359-66.

4. Price, P.A., S.A. Faus, and M.K. Williamson, Warfarin causes rapid calcification of the elastic lamellae in rat arteries and heart valves. Arterioscler Thromb Vasc Biol, 1998. 18(9): p. 1400-7.

5. Price, P.A., A.M. Roublick, and M.K. Williamson, Artery calcification in uremic rats is increased by a low protein diet and prevented by treatment with ibandronate. Kidney Int, 2006. 70(9): p. 1577-83.

6. Wallin, R., et al., Modulation of the binding of matrix Gla protein (MGP) to bone morphogenetic protein-2 (BMP-2). Thromb Haemost, 2000. 84(6): p. 1039-44.

7. Bostrom, K., et al., Matrix GLA protein modulates differentiation induced by bone morphogenetic protein-2 in C3H10T1/2 cells. J Biol Chem, 2001. 276(17): p. 14044-52.

8. Zebboudj, A.F., M. Imura, and K. Bostrom, Matrix GLA protein, a regulatory protein for bone morphogenetic protein-2. J Biol Chem, 2002. 277(6): p. 4388-94.

9. Wajih, N., et al., Processing and transport of matrix gamma-carboxyglutamic acid protein and bone morphogenetic protein-2 in cultured human vascular smooth muscle cells: evidence for an uptake mechanism for serum fetuin. J Biol Chem, 2004. 279(41): p. 43052-60.

10. Luo, G., et al., Spontaneous calcification of arteries and cartilage in mice lacking matrix GLA protein. Nature, 1997. 386(6620): p. 78-81.

11. El-Maadawy, S., et al., Cartilage formation and calcification in arteries of mice lacking matrix Gla protein. Connect Tissue Res, 2003. 44 Suppl 1: p. 272-8.

12. Munroe, P.B., et al., Mutations in the gene encoding the human matrix Gla protein cause Keutel syndrome. Nat Genet, 1999. 21(1): p. 142-4.

13. Keutel, J., G. Jorgensen, and P. Gabriel, A new autosomal-recessive hereditary syndrome. Multiple peripheral pulmonary stenosis, brachytelephalangia, inner-ear deafness, ossification or calcification of cartilages. Dtsch Med Wochenschr, 1971. 96(43): p. 1676-81 passim.

14. Say, B., et al., Unusual calcium deposition in cartilage associated with short stature and peculiar facial features: a case report. Pediatr Radiol, 1973. 1(2): p. 127-9.

15. Walbaum, R., et al., Proceedings: Keutel's syndrome. Pediatrie, 1975. 30(3): p. 313-4. 
16. Fryns, J.P., et al., Calcification of cartilages, brachytelephalangy and peripheral pulmonary stenosis. Confirmation of the Keutel syndrome. Eur J Pediatr, 1984. 142(3): p. 201-3.

17. Cormode, E.J., M. Dawson, and R.B. Lowry, Keutel syndrome: clinical report and literature review. Am J Med Genet, 1986. 24(2): p. 289-94.

18. Khosroshahi, H.E., et al., Keutel syndrome: a report of four cases. Eur J Pediatr, 1989. 149(3): p. $188-91$.

19. Ziereisen, F., C. De Munter, and N. Perlmutter, The Keutel syndrome. Report of a case and review of the literature. Pediatr Radiol, 1993. 23(4): p. 314-5.

20. Haddad, M.C., et al., Premature tracheobronchial, laryngeal and costochondral cartilage calcification in children. Clin Radiol, 1993. 47(1): p. 52-5.

21. Buchsteiner, I., et al., [Congenital subglottic laryngeal stenosis in 2 brothers with chondrodysplasia syndrome (Keutel-Gabriel syndrome)]. Laryngo-rhino-otologie, 1998. 77(7): p. 363-6.

22. Teebi, A.S., et al., Keutel syndrome: further characterization and review. Am J Med Genet, 1998. 78(2): p. 1827.

23. Tuysuz, B., et al., Picture of the month. Keutel syndrome. Arch Pediatr Adolesc Med, 1999. 153(7): p. 765-6.

24. Gilbert, B. and D. Lacombe, Keutel syndrome and miscarriages. Am J Med Genet, 1999. 83(3): p. $209-11$.

25. Miller, S.F., Brachytelephalangy with sparing of the fifth distal phalanx: a feature highly suggestive of Keutel syndrome. Pediatr Radiol, 2003. 33(3): p. 186-9.

26. Hur, D.J., et al., A novel MGP mutation in a consanguineous family: review of the clinical and molecular characteristics of Keutel syndrome. Am J Med Genet A, 2005. 135(1): p. 36-40.

27. Parmar, H., et al., Petrified ears in a patient with Keutel syndrome: temporal bone CT findings. Pediatr Radiol, 2006. 36(3): p. 241-3.

28. Ozdemir, N., et al., Tracheobronchial calcification associated with Keutel syndrome. Turk J Pediatr, 2006. 48(4): p. 357-61.

29. Meier, M., et al., Tracheobronchial stenosis in Keutel syndrome. Eur Respir J, 2001. 17(3): p. 566-9.

30. Schurgers, L.J., et al., Characteristics and performance of an immunosorbent assay for human matrix Glaprotein. Clin Chim Acta, 2005. 351(1-2): p. 131-8.

31. Cranenburg, E.C., et al., Characterisation and potential diagnostic value of circulating matrix Gla protein (MGP) species. Thromb Haemost, 2010. 104(4): p. 811-22.

32. Schurgers, L.J., et al., Vitamin K-containing dietary supplements: comparison of synthetic vitamin $K 1$ and nattoderived menaquinone-7. Blood, 2007. 109(8): p. 3279-83.

33. Al-Qaisi, M., et al., Ankle brachial pressure index (ABPI): An update for practitioners. Vasc Health Risk Manag, 2009. 5: p. 833-41.

34. Jourdan, C., et al., Normative values for intima-media thickness and distensibility of large arteries in healthy adolescents. J Hypertens, 2005. 23(9): p. 1707-15.

35. Gericke, A., et al., Importance of phosphorylation for osteopontin regulation of biomineralization. Calcif Tissue Int, 2005. 77(1): p. 45-54.

36. Boskey, A.L., et al., Osteopontin-hydroxyapatite interactions in vitro: inhibition of hydroxyapatite formation and growth in a gelatin-gel. Bone Miner, 1993. 22(2): p. 147-59.

37. Hunter, G.K., C.L. Kyle, and H.A. Goldberg, Modulation of crystal formation by bone phosphoproteins: structural specificity of the osteopontin-mediated inhibition of hydroxyapatite formation. Biochem J, 1994. 300 ( Pt 3): p. 723-8.

38. Jono, S., C. Peinado, and C.M. Giachelli, Phosphorylation of osteopontin is required for inhibition of vascular smooth muscle cell calcification. J Biol Chem, 2000. 275(26): p. 20197-203.

39. Steitz, S.A., et al., Osteopontin inhibits mineral deposition and promotes regression of ectopic calcification. Am J Pathol, 2002. 161(6): p. 2035-46.

40. Schurgers, L.J., et al., Post-translational modifications regulate matrix Gla protein function: importance for inhibition of vascular smooth muscle cell calcification. J Thromb Haemost, 2007. 5(12): p. 2503-2511. 


\section{Rare mutations in the gene encoding matrix Gla-protein (MGP): \\ a new case of Keutel syndrome serves as a model for exploring MGP functions in humans}

41. Murshed, M., et al., Extracellular matrix mineralization is regulated locally; different roles of two gla-containing proteins. J Cell Biol, 2004. 165(5): p. 625-30.

42. Berkner, K.L. and K.W. Runge, The physiology of vitamin $K$ nutriture and vitamin K-dependent protein function in atherosclerosis. J Thromb Haemost, 2004. 2(12): p. 2118-32.

43. Nanda, A., et al., Keutel syndrome with overlapping features of cutis laxa: a new variant. Am J Med Genet A, 2006. 140(13): p. 1487-9; author reply 1490.

44. Shroff, R.C., et al., Dialysis accelerates medial vascular calcification in part by triggering smooth muscle cell apoptosis. Circulation, 2008. 118(17): p. 1748-57.

45. Speer, M., et al., Inactivation of the osteopontin gene enhances vascular calcification of matrix Gla proteindeficient mice: evidence for osteopontin as an inducible inhibitor of vascular calcification in vivo. J Exp Med, 2002. 196(8): p. 1047-55.

46. Kaartinen, M.T., et al., Osteopontin upregulation and polymerization by transglutaminase 2 in calcified arteries of Matrix Gla protein-deficient mice. J Histochem Cytochem, 2007. 55(4): p. 375-86. 



\section{CHAPTER 7}

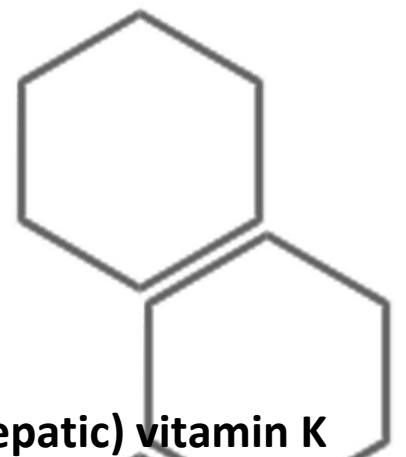

\section{Vitamin $\mathrm{K}$ intake and (hepatic and extra-hepatic) vitamin $\mathrm{K}$}

status in hemodialysis patients: a pilot study

Ellen C.M. Cranenburg ${ }^{1}$, Leon J. Schurgers ${ }^{1}$, Herma Uiterwijk ${ }^{2}$, Joline W.J. Beulens ${ }^{3}$, Gerdien W. Dalmeijer ${ }^{3}$, Ralf Westerhuis ${ }^{2}$, Elke J. Magdeleyns ${ }^{1}$, Marjolein Herfs ${ }^{1}$, Cees Vermeer $^{1}$, G.D. Laverman ${ }^{2,4}$.

${ }^{1}$ VitaK and Cardiovascular Research Institute Maastricht (CARIM), Maastricht University, Maastricht, the Netherlands; ${ }^{2}$ Dialysis Center Groningen, Groningen, the Netherlands; ${ }^{3}$ Julius Center for Health Sciences and Primary Care, University Medical Center Utrecht, Utrecht, the Netherlands; ${ }^{4}$ Department of Internal Medicine, Division of Nephrology, University Medical Center Groningen, University of Groningen, Groningen, the Netherlands 


\begin{abstract}
Vitamin $\mathrm{K}$ is essential for the activity of $\mathrm{\gamma}$-carboxyglutamate ( $\mathrm{Gla}$ )-proteins, including matrix Gla-protein (MGP) and osteocalcin (OC). MGP is a potent local inhibitor of vascular calcification and $\mathrm{OC}$ is the most abundant non-collagenous bone matrix protein. Insufficient intake of vitamin $\mathrm{K}$ will lead to the production of uncarboxylated, inactive proteins. Vitamin $\mathrm{K}$ deficiency could contribute to the high cardiovascular calcification risk in hemodialysis (HD) patients. In this study we investigated vitamin $K_{1}$ and $K_{2}$ intake, as well as vitamin $\mathrm{K}$ status in a cohort of 40 Dutch HD patients.

Vitamin $\mathrm{K}$ intake was calculated using food diaries. Vitamin $\mathrm{K}$ status was assessed using measurements of circulating vitamin K, uncarboxylated MGP, uncarboxylated OC, and protein induced by vitamin $\mathrm{K}$ absence II (PIVKA-II; uncarboxylated prothrombin). Vitamin K intake was low in dialysis patients (median [range]; 140 [30-546] $\mu \mathrm{g} / \mathrm{day}$ ), especially on days of dialysis treatment and weekend days, as compared to intakes reported in Dutch population studies (>225 $\mathrm{\mu g} /$ day). PIVKA-II levels were elevated in $82.5 \%$ of patients, indicating hepatic subclinical vitamin $\mathrm{K}$ deficiency in the majority of patients. In addition, the very high uncarboxylated MGP levels found in all patients suggest vascular vitamin $\mathrm{K}$ deficiency.
\end{abstract}

It is concluded that vitamin $\mathrm{K}$ intake in HD patients is too low for maximal carboxylation of both hepatic and extra-hepatic Gla-proteins. Particularly the high inactive MGP levels may result in an increased risk for arterial calcification. Increasing the intake of vitamin $\mathrm{K}$ may therefore have significant health benefits for HD patients. 


\section{Vitamin K intake and (hepatic and extra-hepatic) vitamin $\mathrm{K}$ status in hemodialysis patients: a pilot study}

\section{Introduction}

Vitamin $\mathrm{K}$ is necessary for the function of proteins containing $\mathrm{\gamma}$-carboxyglutamate (Gla) residues. Well-known vitamin K-dependent proteins (Gla-proteins) are the vitamin Kdependent coagulation factors, which are mainly synthesized in the liver. Extra-hepatic Gla-proteins are osteocalcin (OC), synthesized in bone, and matrix Gla-protein (MGP), synthesized in the vasculature and in cartilage. Gla-residues are formed during a vitamin $\mathrm{K}$-dependent posttranslational carboxylation reaction and are essential for the activity of Gla-proteins [1]. Insufficient dietary intake of vitamin $\mathrm{K}$ will lead to the production of uncarboxylated (i.e. inactive) proteins [2].

MGP synthesized by vascular smooth muscle cells is the strongest physiological inhibitor of vascular calcification currently known. Deficiency of carboxylated MGP may contribute substantially to the development and progression of arterial calcification. Vascular calcifications are found in 60 to $80 \%$ of hemodialysis (HD) patients $[3,4]$ and are associated with a high cardiovascular risk, independent of traditional atherogenic risk factors $[5,6]$. Areas of calcification in vascular tissue are associated with accumulation of uncarboxylated MGP species, which has also been found to precede the development of clinically overt calcification in children on dialysis [7].

Vitamin $\mathrm{K}$ intake may be differentiated for the intake of vitamin $\mathrm{K}_{1}$ (phylloquinone) and vitamin $K_{2}$ (group name for menaquinones). The estimated daily vitamin $K$ intake in a Western diet ranges from 60 to $200 \mu \mathrm{g}$, which is mainly determined by vitamin $\mathrm{K}_{1}$ (phylloquinone) intake. Vitamin $\mathrm{K}_{1}$ intakes reported in the Netherlands are typically higher (>200 $\mu \mathrm{g} /$ day) [8-13]. Vitamin $\mathrm{K}_{1}$ and vitamin $\mathrm{K}_{2}$ content of food products in the Netherlands has been extensively studied by our laboratory in the past [14], resulting in a comprehensive dietary vitamin $K_{1}$ and $K_{2}$ database. Previous studies using this database demonstrated that intake of vitamin $K_{2}$ was inversely associated with cardiovascular calcification and mortality $[10,12,13]$.

Systemic vitamin $\mathrm{K}$ status can be determined by 1 ) measuring circulating vitamin $\mathrm{K}_{1}$ and $\mathrm{K}_{2}$ levels and 2) by measuring circulating inactive forms of vitamin K-dependent proteins. Intake of vitamin K-containing food products will readily influence measurements of circulating vitamin $\mathrm{K}$ levels. In contrast, measurements of uncarboxylated prothrombin (known as protein induced by vitamin $\mathrm{K}$ absence/antagonism II [PIVKA-II]), uncarboxylated OC, and dp-ucMGP will reflect utilization of vitamin $\mathrm{K}$ in tissues. We recently developed a new MGP assay, measuring desphospho-uncarboxylated (dp-uc) MGP species. First results show that this inactive, immature MGP species is suited to assess extra-hepatic vitamin $\mathrm{K}$ status, particularly in the vasculature [15]. 
In the present study, we therefore aimed to investigate vitamin $\mathrm{K}_{1}$ and $\mathrm{K}_{2}$ intake as well as vitamin $\mathrm{K}$ status in $\mathrm{HD}$ patients. Vitamin $\mathrm{K}$ intake was estimated using a comprehensive dietary vitamin $\mathrm{K}$ database, which has been previously used in Dutch population studies $[12,13]$. Vitamin $\mathrm{K}$ status was evaluated with measurements of circulating vitamin $\mathrm{K}_{1}$ and $\mathrm{K}_{2}$ levels, as well as of hepatic and extra-hepatic vitamin $\mathrm{K}$-dependent proteins, including PIVKA-II, MGP, and OC. With the present study we expected to obtain new insights in different aspects of vitamin $\mathrm{K}$ metabolism in HD patients, and to increase our knowledge on the risk factors and pathogenesis of arterial calcification in these patients. Additionally, the results of this study will give an indication whether HD patients may benefit from an increased dietary vitamin $\mathrm{K}$ intake. 


\section{Vitamin K intake and (hepatic and extra-hepatic) vitamin $\mathrm{K}$ status in hemodialysis patients: a pilot study}

\section{Methods}

\section{Patients}

For this explorative observational study, patients with end-stage renal disease who had been on regular HD treatment at the Dialysis Center Groningen for at least 3 months, were invited to participate. Patients with a minimum age of 18 years were eligible, irrespective of gender, the underlying primary renal disease, the presence of cardiovascular disease (CVD), diabetes mellitus (DM), or traditional atherogenic risk factors. Malignancy in the past 6 months, abnormal liver function, a history of gastrointestinal disease or metabolic disease, or an active infection were exclusion criteria. Additional exclusion criteria were use of coumarin derivatives, anticonvulsants (such as phenytoin, carbamazepine, and phenobarbital), which may influence vitamin $\mathrm{K}$ metabolism, and use of vitamin $\mathrm{K}$ supplements. Data were obtained on demographic, clinical, and dialysis-specific characteristics during interview and from review of patient's files. Complete data were acquired from 40 patients. Informed consent was obtained from all participants and the study was approved by the local Medical Ethics Committee.

\section{Vitamin K intake}

Energy and nutrient intake were estimated by registration of all food items consumed, including beverages, and the amount consumed during 4 non-consecutive days in food diaries by the patients, within a time-period of 2 weeks. All participants received verbal and written instructions before receiving the food diary. The diary consisted of 2 week days and 1 weekend day, during which the patient did not undergo HD treatment, and 1 week day during which the patient underwent HD treatment (dialysis day).

Diaries were analyzed using a nutrient software program (part of Evry dietist, Ensemble BV, Zoetermeer, the Netherlands), which is based on the Dutch national food compositions (NEVO) table. Energy and nutrient intake were calculated using the 2006 version of the NEVO table [16]. Concentrations of vitamin $\mathrm{K}_{1}$ and $\mathrm{K}_{2}$ (MK-4 through MK-10) of 260 foods have been added to the NEVO (2006) food database, as described previously [13]. All nutrients were adjusted for total energy intake by using the residual method [17]. Due to the variance intake on week, weekend, and dialysis days, intake was measured on nonconsecutive days on 1 dialysis day, 2 separate week days, and 1 weekend day. These intake measurements were weighted to reflect their frequence in a 2-week period. This resulted in the following weightings: $6 / 14$ for weekdays, $3 / 14$ for weekend days, and 5/14 for dialysis days. In our study population, vegetables contributed $71 \%$ of vitamin $\mathrm{K}_{1}$ intake, cheese contributed $59 \%$, milk products $14 \%$, and meat $18 \%$ of vitamin $\mathrm{K}_{2}$ intake. 


\section{Vitamin K status}

Vitamin $\mathrm{K}$ status was assessed by measurements of circulating vitamin $\mathrm{K}_{1}$ and $\mathrm{K}_{2}$ (menaquinones (MK)-4 through MK-10), and the vitamin K-dependent proteins PIVKA-II, dpUCMGP, UCOC, and COC. Venous blood samples were taken before the initiation of a HDsession after the two-day dialysis-free interval. Participants were asked to adhere to their dietary habits, but to refrain from consumption of vitamin K-containing food items (e.g. green vegetables and fermented food products) the evening before and the day of blood withdrawal. Before serum preparation, blood was kept for $20 \mathrm{~min}$ at room temperature. Plasma and serum were prepared by standard centrifugation and stored at $-80^{\circ} \mathrm{C}$ until testing.

The circulating concentrations of vitamin $\mathrm{K}$ species (vitamin $\mathrm{K}_{1}$, MK-4 through MK-10) were measured using reversed phase high-performance liquid chromatography as described previously [14].

PIVKA-II levels were measured with the ASSERACHROM PIVKA-II kit (Diagnostica Stago, Asnières-sur-Mer, France). This enzyme-linked immunosorbent assay (ELISA) is based on mouse monoclonal $F\left(a b^{\prime}\right) 2$ fragments specific for PIVKA-II, without reactivity with native prothrombin.

Circulating dp-ucMGP levels were determined in plasma using a dual-antibody ELISA (VitaK BV, Maastricht, the Netherlands). In this assay, the capture antibody is directed against the non-phosphorylated MGP sequence 3-15 and the detection antibody against the uncarboxylated MGP sequence 35-49. This assay was shown to be particularly suited to assess vascular vitamin $\mathrm{K}$ status [15].

Markers used to evaluate the carboxylation status of circulating osteocalcin were uncarboxylated osteocalcin (ucOC), carboxylated osteocalcin ( $\mathrm{COC}$ ), and the ratio between ucOC and $\mathrm{COC}(\mathrm{ucOC} / \mathrm{cOC})$. Both ucOC and $\mathrm{cOC}$ levels were measured with ELISAs based conformation-specific OC antibodies (Takara, Shiga, Japan).

Other biochemical measurements, including calcium, phosphorus, cholesterol, and triglycerides, were performed using standard laboratory techniques. 


\section{Vitamin K intake and (hepatic and extra-hepatic) vitamin $\mathrm{K}$ status in hemodialysis patients: a pilot study}

Statistical analysis

Normally and non-normally distributed continuous variables are presented as mean \pm standard deviation (SD) or as median [range], respectively, and categorical variables as numbers and frequencies. Differences in vitamin $\mathrm{K}$ intake between day types (week, weekend, and dialysis days) were analyzed with Kruskal-Wallis and Mann-Whitney $\mathrm{U}$ tests. Within-group differences in vitamin $\mathrm{K}$ intake and parameters of vitamin $\mathrm{K}$ status were analyzed with Kruskal-Wallis and Mann-Withney $U$ tests. Bonferroni correction was applied for multiple comparisons. Bivariate correlation analysis was performed using the Pearson correlation coefficient for normally distributed data and Spearman correlation coefficient for non-normally distributed data. A pvalue of $\leq 0.05$ was considered to be statistically significant; reported $\mathrm{p}$ values are based on two-tailed tests of statistical significance. All statistical analyses were conducted using SPSS version 15.0 for Windows (SPSS Corp, Chicago, IL, USA). 


\section{Results}

\section{Patient population}

Vitamin $\mathrm{K}$ intake and status were investigated in the entire cohort of $40 \mathrm{HD}$ patients, consisting of 22 male and 18 female patients with a mean age of $62 \pm 16$ years. The median [range] dialysis vintage was 40 [4-385] months. All baseline characteristics of the patient population are presented in Table 1. As expected, the prevalence of CVD and hypertension was high. Control of calcium and phosphorus levelswas quite well. .

\section{Vitamin K intake}

Total vitamin $\mathrm{K}$ intake of patients, registered during 4 days, was 140 [30-546] $\mu \mathrm{g} /$ day, consisting of 118 [18-494] $\mu \mathrm{g} /$ day vitamin $\mathrm{K}_{1}$ and 21 [2-68] $\mu \mathrm{g} /$ day vitamin $\mathrm{K}_{2}$ (Table 2). As expected, total vitamin $\mathrm{K}$ intake was predominantly determined by intake of vitamin $\mathrm{K}_{1}$. One-third of the patients $(n=13,33 \%)$ had an average total vitamin $K$ intake of less than $100 \mu \mathrm{g} /$ day. Total vitamin $\mathrm{K}$ intake was considerably lower on weekend days and dialysis days (Table 2 ), as compared to week days ( $p=0.003$ for both comparisons). These differences originated from differences in vitamin $K_{1}$ intake between these day-types $(p=0.003$ for both comparisons), without significant differences in vitamin $\mathrm{K}_{2}$ intake.

There were no differences in total vitamin $\mathrm{K}, \mathrm{K}_{1}$, or $\mathrm{K}_{2}$ intake between males and females, or between patients with CVD or DM and patients without these conditions (Table 2). In correlation analysis, total vitamin $\mathrm{K}$ intake was not associated with age, BMI, dialysis vintage, or any of the biochemical characteristics. 


\section{Vitamin K intake and (hepatic and extra-hepatic) vitamin K status in hemodialysis patients: a pilot study}

Table 1: Characteristics of the $\mathbf{4 0}$ HD patients

\begin{tabular}{|c|c|}
\hline Demographic and clinical characteristics & HD population $(n=40)$ \\
\hline Age (years) & $65(23-86)$ \\
\hline Sex (male/female ratio) & $22 / 18$ \\
\hline Dialysis vintage (months) & $40(4-385)$ \\
\hline \multicolumn{2}{|l|}{ Presumed cause of ESRD (number of patients [\%]) } \\
\hline Glomerular disease & $9[23]$ \\
\hline Vascular disease, Hypertension & $10[25]$ \\
\hline Polycystic kidney disease & $9[23]$ \\
\hline Diabetic kidney disease & $2[5]$ \\
\hline Miscellaneous & $6[15]$ \\
\hline Unknown & $4[10]$ \\
\hline Hypertension (number of patients [\%]) & $27[67.5]$ \\
\hline Diabetes (number of patients [\%]) & $6[15.0]$ \\
\hline Current smoking (number of patients [\%]) & $6[15.0]$ \\
\hline BMI $\left(\mathrm{kg} / \mathrm{m}^{2}\right)$ & $24.6 \pm 3.7$ \\
\hline Cardiovascular disease (number of patients [\%]) & $15[37.5]$ \\
\hline Coronary artery disease & $10[25.0]$ \\
\hline Peripheral artery disease & $5[12.5]$ \\
\hline Cerebrovascular disease & $4[10]$ \\
\hline History of renal transplantation & $7[17.5]$ \\
\hline History of parathyroidectomy (number of patients [\%]) & $5[12.5]$ \\
\hline \multicolumn{2}{|l|}{ Biochemical characteristics } \\
\hline $\mathrm{Hb}(\mathrm{mmol} / \mathrm{L})$ & $7.2 \pm 0.8$ \\
\hline $\mathrm{Ca}(\mathrm{mmol} / \mathrm{L})$ & $2.3 \pm 0.2$ \\
\hline $\mathrm{P}(\mathrm{mmol} / \mathrm{L})$ & $1.6 \pm 0.3$ \\
\hline $\mathrm{Ca} \times \mathrm{P}\left(\mathrm{nmol}{ }^{2} / \mathrm{L}^{2}\right)$ & $3.6 \pm 0.8$ \\
\hline $\operatorname{AP}(U / L)$ & $72 \pm 23$ \\
\hline PTH (pmol/L) & $15(1-94)$ \\
\hline Cholesterol (mmol/L) & $4.0 \pm 1.0$ \\
\hline Triglycerides (mmol/L) & $1.9 \pm 0.8$ \\
\hline Vitamin D supplementation (number of patients [\%]) & $37(92.5 \%)$ \\
\hline Cinacalcet (number of patients [\%]) & $4(10)$ \\
\hline Oral PB (number of patients [\%]) & $35(87.5)$ \\
\hline$>1$ oral PB & $20(50)$ \\
\hline Ca-containing PB & $16(40)$ \\
\hline Non-Ca containing PB & $29(72.5)$ \\
\hline Antihypertensive medication (number of patients [\%]) & $28(70)$ \\
\hline$>1$ medication & $10(25)$ \\
\hline Iron injections (number of patients [\%]) & $38(95)$ \\
\hline Darbepoetin alfa (number of patients [\%]) & $37(92.5)$ \\
\hline \multicolumn{2}{|l|}{ Dietary intake } \\
\hline Total energy $(\mathrm{kJ} / \mathrm{d})$ & $7502 \pm 2119$ \\
\hline Total protein $(\mathrm{g} / \mathrm{d})$ & $66 \pm 8.5$ \\
\hline Total fat $(\mathrm{g} / \mathrm{d})$ & $62 \pm 9$ \\
\hline Mono-unsaturated fat (g/d) & $21 \pm 4$ \\
\hline Poly-unsaturated fat (g/d) & $11 \pm 3$ \\
\hline Saturated fat $(g / d)$ & $26 \pm 5$ \\
\hline Fiber $(g / d)$ & $20 \pm 12$ \\
\hline Calcium (mg/d) & $814 \pm 235$ \\
\hline Vitamin C (mg/d) & $68 \pm 31$ \\
\hline
\end{tabular}


Data are given as mean $\pm \mathrm{SD}$, median [range], or as absolute numbers with percentage of total between parentheses. Cardiovascular disease was defined as a history of coronary artery disease (myocardial infarction, angina pectoris or evidence of obstructive disease by angiography), cerebrovascular disease (thrombotic stroke or transient ischemic attack), calcific aortic valve disease, or peripheral artery disease (a history of claudication or lower extremity revascularization). Abbreviations used: BMI, body mass index; Ca, calcium; P, phosphorus.

Table 2: Vitamin K intake

\begin{tabular}{|c|c|c|c|c|}
\hline \multicolumn{5}{|c|}{ Intake ( $\mu \mathrm{g} /$ day) of energy-adjusted vitamin K } \\
\hline & & Total & Vitamin $K_{1}$ & Vitamin $\mathrm{K}_{2}$ \\
\hline \multicolumn{5}{|l|}{ Average all days } \\
\hline & & $140[30-546]$ & 118 [18-494] & $21[2-68]$ \\
\hline \multicolumn{5}{|l|}{ Day types } \\
\hline Week (Monday-Friday) & & $171[41-888]$ & & \\
\hline Weekend (Saturday, Sunday) & & $53[13-1075]$ & & \\
\hline \multirow[t]{2}{*}{ Hemodialysis } & & $66[5-452]$ & & \\
\hline & $\mathbf{p}$ & $<0.001$ & $<0.001$ & 0.624 \\
\hline \multicolumn{5}{|l|}{ Patients } \\
\hline Male $(n=22)$ & & 115 [42-393] & 96 [33-360] & $19[2-48]$ \\
\hline \multirow[t]{2}{*}{ Female $(n=18)$} & & $178[30-546]$ & 144 [18-494] & $22[4-68]$ \\
\hline & $\mathbf{p}$ & 0.289 & 0.302 & 0.471 \\
\hline with CVD ( $n=15)$ & & $113[30-546]$ & $93[18-494]$ & $18[3-52]$ \\
\hline \multirow[t]{2}{*}{ without CVD $(n=25)$} & & $162[42-413]$ & 134 [18-385] & $22[2-68]$ \\
\hline & $\mathbf{p}$ & 0.121 & 0.158 & 0.451 \\
\hline with DM & & 139 [66-292] & $107[47-272]$ & 19 [4-48] \\
\hline \multirow[t]{2}{*}{ without DM } & & $140[30-546]$ & $123[18-494]$ & $22[2-68]$ \\
\hline & $\mathbf{p}$ & 0.791 & 0.910 & 0.691 \\
\hline
\end{tabular}

Vitamin $\mathrm{K}$ intakes (total, $\mathrm{K}_{1}$, and $\mathrm{K}_{2}$ ) are given for different day types (week, weekend, and dialysis) and for different patient groups (female and male, with and without cardiovascular disease and diabetes mellitus). Data are given as median [range]. P-values are based on Kurskal-Wallis tests (for comparison of day-types) and Mann-Whitney $U$ tests (for comparison of patient groups). Abbreviations used: CVD, cardiovascular disease; DM, diabetes mellitus.

\section{Vitamin K status}

Serum vitamin $\mathrm{K}_{1}$ concentration showed a broad range (Table 3 ). Almost half of patients $(n=18 ; 45 \%)$ had circulating vitamin $K_{1}$ levels below the normal range. Of the menaquinones (vitamin $\mathrm{K}_{2}$ ), only MK-4 was measurable in low quantities in the blood. MK-5 through MK-10 were undetectable in the circulation of the patients (Table 3). Hepatic vitamin $\mathrm{K}$ status, as assessed with PIVKA-II measurement, was suboptimal in the patient cohort. Thirty-three patients (82.5\%) had elevated PIVKA-II levels. The median level of 3.8 [1.4-12.4] $\mathrm{ng} / \mathrm{ml}$ was almost twice the upper normal level. Plasma PIVKA-II levels were not associated with any of the baseline characteristics. 


\section{Vitamin $\mathrm{K}$ intake and (hepatic and extra-hepatic) vitamin $\mathrm{K}$ status in hemodialysis patients: a pilot study}

\begin{tabular}{|c|c|c|}
\hline Vitamin K status & HD patients & Reference values \\
\hline \multicolumn{3}{|c|}{ 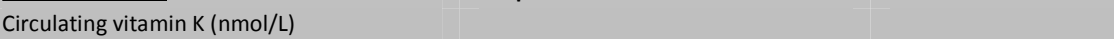 } \\
\hline Vitamin $\mathrm{K}_{1}$ & $0.30[0.00-5.71]$ & $0.029-2.65$ \\
\hline MK-4 & $0.00[0.00-1.13]$ & $<$ lower detection limit \\
\hline MK-5 trough MK-10 & $0.00[0.00-0.00]$ & $<$ lower detection limit \\
\hline \multicolumn{3}{|l|}{ Hepatic vitamin $\mathrm{K}$ status } \\
\hline PIVKA-II (ng/ml) & $3.8[1.4-12.4]$ & $<2$ \\
\hline \multicolumn{3}{|c|}{ Extra-hepatic vitamin $\mathrm{K}$ status } \\
\hline dp-ucMGP (pmol/l) & $1868 \pm 870$ & $50-700$ \\
\hline $\mathrm{ucOC}(\mathrm{ng} / \mathrm{ml})$ & $6.1 \pm 28$ & $2-4$ \\
\hline $\mathrm{cOC}(\mathrm{ng} / \mathrm{ml})$ & $11.7 \pm 2.8$ & $5-9$ \\
\hline ratio ucOC/cOC & $0.5[0.1-0.9]$ & $<1.0$ \\
\hline
\end{tabular}

Values of different markers for vitamin $\mathrm{K}$ status are given: circulating vitamin $\mathrm{K}_{1}$ and $\mathrm{K}_{2}$ levels, PIVKA-II levels for hepatic vitamin $\mathrm{K}$ status, and dp-ucMGP and OC levels for extra-hepatic vitamin $\mathrm{K}$ status. Reference values in healthy subjects are given based on literature (vitamin $K_{1}$ and $K_{2}$ levels), information provided by manufacturer of the assay (PIVKA-II), and measurements in healthy subjects aged above 40 years (dp-ucMGP and OC). Data are given as mean \pm SD or median [range].

Extra-hepatic vitamin $\mathrm{K}$ status was assessed by dp-ucMGP and OC measurements, reflecting vascular and bone vitamin $\mathrm{K}$ status, respectively. Plasma dp-ucMGP levels were elevated in all 40 patients (Table 3 ), indicating suboptimal vascular vitamin $\mathrm{K}$ status in the entire patient cohort. The dp-ucMGP levels were positively correlated with dialysis vintage (Spearman's rho=0.440, $\mathrm{p}=0.035$ ). In addition, patients with CVD had significantly higher dp-ucMGP levels $(2226 \pm 1103 \mathrm{pmol} / \mathrm{L})$ compared to patients without CVD $(1653 \pm 626 \mathrm{pmol} / \mathrm{L} ; \mathrm{p}=0.034)$. The mean $\mathrm{ucOC}$ level was considerably increased compared with average levels found in healthy reference subjects aged $>40$ years (Table 3 ). However, since cOC levels were also elevated, the average ucOC/cOC ratio was 0.5 , which is within the normal range.

When investigating the associations between the different parameters for vitamin $\mathrm{K}$ status, plasma dp-ucMGP levels were positively correlated with PIVKA-II levels (Spearman's rho $=0.384, p=0.028$ ), depicted in Figure 1 . There were no other significant correlations. Investigation of the associations between vitamin $\mathrm{K}$ intake (total, $\mathrm{K}_{1}$, and $\mathrm{K}_{2}$ intake) and measurements of vitamin $\mathrm{K}$ status, revealed no significant associations. 
Figure 1: Correlation between dp-ucMGP and PIVKA-II measurements

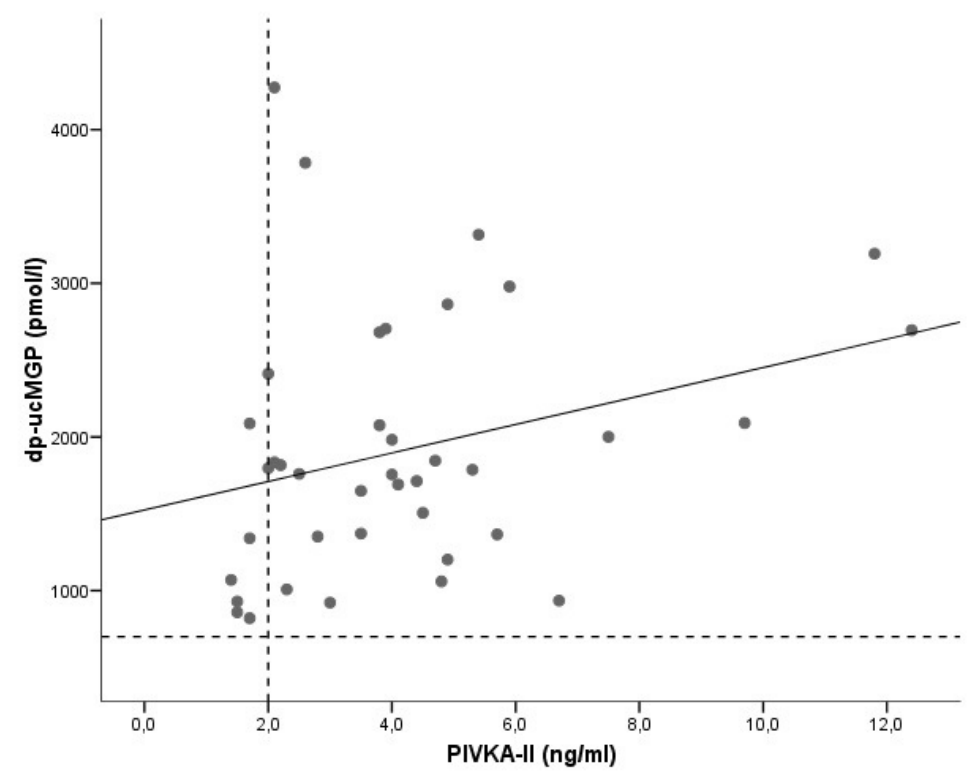

Graph depicting the correlation between dp-ucMGP and PIVKA-II measurements (Spearman's rho=0.384, $\mathrm{p}=0.028)$. The dotted lines indicate the reference values of circulating dp-ucMGP $(<700 \mathrm{pmol} / \mathrm{L})$ and PIVKA-II $(<2$ $\mathrm{ng} / \mathrm{ml}$ ). It can be clearly seen that the majority of patients had elevated dp-ucMGP as well as PIVKA-II levels. 


\section{Vitamin K intake and (hepatic and extra-hepatic) vitamin $\mathrm{K}$ status in hemodialysis patients: a pilot study}

\section{Discussion}

This is the first study fully assessing vitamin $\mathrm{K}$ intake and vitamin $\mathrm{K}$ status in HD patients. The main finding was that vitamin $\mathrm{K}$ intake in the present $\mathrm{HD}$ patient cohort was relatively low. The second important finding is that both hepatic as well as extra-hepatic vitamin $\mathrm{K}$ status were strikingly poor, with the most extreme situation for the vascular wall.

Dietary intake of vitamin $\mathrm{K}$ in patients was below values previously found in our country (>225 $\mu \mathrm{g} /$ day) [8-13]. Low vitamin $\mathrm{K}$ intake of HD patients may be related to the dietary regimen generally prescribed for HD patients, which includes restriction of sodium and potassium intake [18]. Sodium is present in cheese and potassium is abundantly present in green vegetables, which are the food products containing abundant amounts of vitamin $K_{1}$ and $K_{2}$, respectively. Nevertheless, vitamin $\mathrm{K}$ intake of our patients was still higher than might have been expected since it was previously predicted that a typical renal diet would contain $80 \mu \mathrm{g}$ or less of vitamin $\mathrm{K}$ per day [19]. This may have been be related to a relatively high green vegetable and cheese intake in the Netherlands. Vitamin $\mathrm{K}$ intake was lower on weekend days and dialysis days as compared to week days, reflecting a different dietary pattern on these days. Consistent with the literature, intake of vitamin $\mathrm{K}_{2}$ seems low compared to that of vitamin $\mathrm{K}_{1}$ [8-13]. However, intake of vitamin $\mathrm{K}_{2}$ is probably highly relevant for cardiovascular health. Vitamin $K_{2}$ intake (and not vitamin $K_{1}$ ) was inversely associated with cardiovascular calcification and mortality in the general population $[10,12,13]$. Vitamin $\mathrm{K}_{2}$ is more uniformly distributed over the various tissues, whereas vitamin $\mathrm{K}_{1}$ is preferentially targeted to the liver [20]. It has been shown for instance, that there is a higher accumulation and utilization of MK-4 in the vascular wall compared to vitamin $\mathrm{K}_{1}$ [21].

Vitamin $\mathrm{K}$ status was investigated using measurements of circulating vitamin K, PIVKA-II, $\mathrm{dp}$-ucMGP, and OC. Circulating vitamin $\mathrm{K}$ levels were below the normal range in almost half of the patients. The majority of the menaquinones (MK-5 through MK-13) were below the lower detection limit, which is usually found in humans [22]. It should be kept in mind that circulating vitamin $\mathrm{K}$ concentrations fluctuate, among others influenced by recent dietary intake. Also circulating vitamin $\mathrm{K}$ may not reflect the utilization of vitamin $\mathrm{K}$ in tissue. The high PIVKA-II levels found in the vast majority of patients indicate that vitamin $\mathrm{K}$ availability in the liver is too low to produce fully carboxylated clotting factors. Although an increased PIVKA-II level may not result in clinical haemostatic problems, it indicates that the hepatic vitamin $\mathrm{K}$ status is poor. The high dp-ucMGP levels found in all patients confirm previous findings in $\mathrm{HD}$ patients $[15,23]$ and indicate a poor vascular vitamin $\mathrm{K}$ status, which may well be a contributing factor in cardiovascular calcification. 
Plasma dp-ucMGP was the only marker that correlated with PIVKA-II levels, stressing its value as a vascular biomarker for vitamin $\mathrm{K}$ status. With respect to osteocalcin, both the ucOC and cOC levels were high, resulting in a normal osteocalcin ratio. This may be explained by retention of circulating OC (fragments) in uremic serum [24, 25]. An alternative explanation may be that ESRD associated increased bone turnover resulted in an overall increased $O C$ synthesis. Further investigations are needed to elucidate why both ucOC and $\mathrm{COC}$ levels measured with ELISAs are high in HD patients. Overall, the application of OC measurements in HD patients seems to be complicated and OC carboxylation may not be a useful marker for vitamin $\mathrm{K}$ status in HD patients [25].

In general, dietary vitamin $\mathrm{K}$ intake in healthy subjects and in HD patients is sufficient to maintain normal haemostasis, but may not be sufficient for full carboxylation (activation) of the extra-hepatic Gla-proteins. Booth and coworkers showed in a cohort of $142 \mathrm{HD}$ patients that vitamin $\mathrm{K}$ status in this patient population is suboptimal [25]. Low circulating vitamin $\mathrm{K}_{1}$ levels and poor OC carboxylation were found in $29 \%$ and $93 \%$ of subjects, respectively. A recent study by the same group in 172 subjects with stage 3 to 5 CKD, showed that the criteria for subclinical vitamin $\mathrm{K}$ deficiency were met by $6 \%$ of the patients based on circulating $\mathrm{K}_{1}$ measurements, by $60 \%$ based on OC carboxylation, and by $97 \%$ based on PIVKA-II levels [26]. The present study confirms these findings, with exception of poor OC carboxylation. It should be noted, however, that OC carboxylation was measured with a different technique in these studies (a radio-immunoassay based on the different affinities of ucOC and COC for hydroxyapatite) than used in the present study (ELISA based on monoclonal antibodies against ucOC and $\mathrm{COC}$ ). Their outcomes may therefore not be comparable with ours. Due to its lipophilic charactertistics and incorporation into lipoproteins, vitamin $\mathrm{K}$ is not expected to be removed by HD treatment [8]. It can therefore be hypothesized that vitamin $\mathrm{K}$ deficiency in HD patients is due to a diminished dietary intake, which may increase the risk of arterial calcification. Our study confirms that the dietary vitamin $\mathrm{K}$ intake in HD patients is low, although we could not demonstrate a correlation between vitamin $\mathrm{K}$ intake and markers for vitamin $\mathrm{K}$ status. This might be due to the relatively low number of patients.

The recommended daily allowance (RDA) for vitamin $\mathrm{K}$ of $1 \mu \mathrm{g} / \mathrm{day} / \mathrm{kg}$ bodyweight is based on intakes of vitamin $\mathrm{K}_{1}$ needed to maintain adequate synthesis of blood coagulation factors. The (higher) requirements of extra-hepatic tissues for maximal carboxylation of Gla-proteins were not taken into account in this recommendation, and although vitamin $\mathrm{K}$ intake met this intake level for the majority of patients, it is clear that it is too low for maximal carboxylation of extra-hepatic Gla-proteins. Based on the elevated plasma levels of PIVKA-II it may even be concluded that the hepatic vitamin K status was not adequate to fully support the carboxylation of the vitamin K-dependent clotting factors. 


\section{Vitamin K intake and (hepatic and extra-hepatic) vitamin $\mathrm{K}$ status in hemodialysis patients: a pilot study}

The number of patients included in our study is too low to investigate whether factors other than low dietary intake may cause insufficient vitamin $\mathrm{K}$ status and to evaluate its long-term consequences. Further studies need to be performed to confirm the present results in larger cohorts of CKD and dialysis patients, including peritoneal dialysis patients. Also, it may be relevant to evaluate kidney transplant recipients, because these patients may well maintain their previous dietary habits as dialysis patient.

What may be the clinical implications of our findings? Improvement of vitamin $\mathrm{K}$ status in HD patients may readily be achieved by food supplements. The bioavailability of vitamin $\mathrm{K}$ from supplements is probably better than that from most foods [22]. For OC it is known that, although $\mathrm{uCOC}$ is responsive to increased dietary vitamin $\mathrm{K}$, a maximal response can only been achieved with pharmacological vitamin $K$ intakes [22]. The most common vitamin $\mathrm{K}$ form in food supplements and multivitamins is $\mathrm{K}_{1}$. Two commercially available $\mathrm{K}_{2}$ vitamins are MK-4 and MK-7. The half-life of MK-7 (approximately 3 days), is much longer compared to that of MK-4 and $K_{1}$, with a steady state plasma level reached after 2 weeks of daily supplementation [27]. In a pilot study among 53 HD patients, the administration of MK-7 (dosage 45, 135, or $360 \mu \mathrm{g} /$ day) resulted in significantly decreased ucOC and dpucMGP levels within 6 weeks (Westenfeld R et al.: ASN Renal Week 2008, Abstract Book). In the highest dosage-group, the response-to-treatment for dp-ucMGP levels was even $100 \%$. To improve the vitamin $\mathrm{K}$ status of HD patients, supplementation may be indeed be the best method, since increasing the dietary intake of vitamin $\mathrm{K}$ is probably not possible due to the generally prescribed dietary regimen, as discussed previously. Since HD patients are generally prescribed multivitamins, it may be relatively easy to add vitamin $\mathrm{K}$ to these preparations. A contra-indication for vitamin $\mathrm{K}$ supplementation is the use of vitamin K-antagonists (oral anticoagulants).

In conclusion, this is the first study demonstrating that HD patients have a vitamin $\mathrm{K}$ intake too low to prevent poor overall vitamin $\mathrm{K}$ status. The high dp-ucMGP levels are strongly suggestive for vascular vitamin $\mathrm{K}$ insuffiency to a level that the calcification-inhibitory activity of MGP is impaired, which may contribute to the extremely high risk for arterial calcification in HD patients. These data therefore warrant vitamin $\mathrm{K}$ intervention studies to elucidate the potential clinical benefit of vitamin $\mathrm{K}$ supplementation in HD patients. 


\section{Acknowlegdements}

The authors would like to thank Diane ter Doest for excellent work in calculating vitamin $\mathrm{K}$ intakes. The authors are grateful to all patients who participated in this study.

\section{References}

1. Vermeer, C., Gamma-carboxyglutamate-containing proteins and the vitamin K-dependent carboxylase. Biochem J, 1990. 266(3): p. 625-36.

2. Berkner, K.L. and K.W. Runge, The physiology of vitamin K nutriture and vitamin K-dependent protein function in atherosclerosis. J Thromb Haemost, 2004. 2(12): p. 2118-32.

3. Goodman, W.G., et al., Coronary-artery calcification in young adults with end-stage renal disease who are undergoing dialysis. N Engl J Med, 2000. 342(20): p. 1478-83.

4. Raggi, P., et al., Cardiac calcification in adult hemodialysis patients. A link between end-stage renal disease and cardiovascular disease? J Am Coll Cardiol, 2002. 39(4): p. 695-701.

5. Blacher, J., et al., Arterial calcifications, arterial stiffness, and cardiovascular risk in end-stage renal disease. Hypertension, 2001. 38(4): p. 938-42.

6. London, G.M., et al., Arterial media calcification in end-stage renal disease: impact on all-cause and cardiovascular mortality. Nephrol Dial Transplant, 2003. 18(9): p. 1731-40.

7. Shroff, R.C., et al., Dialysis accelerates medial vascular calcification in part by triggering smooth muscle cell apoptosis. Circulation, 2008. 118(17): p. 1748-57.

8. Krueger, T., et al., Vitamin $K$ deficiency in CKD patients: a modifiable risk factor for vascular calcification? Kidney Int, 2009. 76(1): p. 18-22.

9. Jie, K.S., et al., Vitamin $K$ intake and osteocalcin levels in women with and without aortic atherosclerosis: a population-based study. Atherosclerosis, 1995. 116(1): p. 117-23.

10. Geleijnse, J.M., et al., Dietary intake of menaquinone is associated with a reduced risk of coronary heart disease: the Rotterdam Study. J Nutr, 2004. 134(11): p. 3100-5.

11. Maas, A.H., et al., Vitamin K intake and calcifications in breast arteries. Maturitas, 2007. 56(3): p. 273-9.

12. Gast, G.C., et al., A high menaquinone intake reduces the incidence of coronary heart disease. Nutr Metab Cardiovasc Dis, 2009. 19(7): p. 504-10.

13. Beulens, J.W., et al., High dietary menaquinone intake is associated with reduced coronary calcification. Atherosclerosis, 2009. 203(2): p. 489-93.

14. Schurgers, L.J. and C. Vermeer, Determination of phylloquinone and menaquinones in food. Effect of food matrix on circulating vitamin $K$ concentrations. Haemostasis, 2000. 30(6): p. 298-307.

15. Cranenburg, E.C., et al., Characterisation and potential diagnostic value of circulating matrix Gla protein (MGP) species. Thromb Haemost. 104(4): p. 811-22.

16. Nederlands Voedingsstoffenbestand, Den Haag, Dutch. Voorlichtinsbureau voor de voeding, 2006.

17. Willett, W.C., G.R. Howe, and L.H. Kushi, Adjustment for total energy intake in epidemiologic studies. Am J Clin Nutr, 1997. 65(4 Suppl): p. 1220-8.

18. Beto, J.A. and V.K. Bansal, Medical nutrition therapy in chronic kidney failure: integrating clinical practice guidelines. J Am Diet Assoc, 2004. 104(3): p. 404-9.

19. Stein, G., H. Sperschneider, and S. Koppe, Vitamin levels in chronic renal failure and need for supplementation. Blood purification, 1985. 3(1-3): p. 52-62.

20. Cranenburg, E.C., L.J. Schurgers, and C. Vermeer, Vitamin K: The coagulation vitamin that became omnipotent. Thromb Haemost, 2007. 98(1): p. 120-5. 


\section{Vitamin K intake and (hepatic and extra-hepatic) vitamin K status in hemodialysis patients: a pilot study}

21. Spronk, H.M., et al., Tissue-specific utilization of menaquinone-4 results in the prevention of arterial calcification in warfarin-treated rats. J Vasc Res, 2003. 40(6): p. 531-7.

22. Vermeer, C., et al., Beyond deficiency: potential benefits of increased intakes of vitamin $K$ for bone and vascular health. Eur J Nutr, 2004. 43(6): p. 325-35.

23. Schurgers, L.J., et al., The circulating inactive form of matrix gla protein is a surrogate marker for vascular calcification in chronic kidney disease: a preliminary report. Clin J Am Soc Nephrol, 2010. 5(4): p. 568-75.

24. Urena, P. and M.C. De Vernejoul, Circulating biochemical markers of bone remodeling in uremic patients. Kidney Int, 1999. 55(6): p. 2141-56.

25. Pilkey, R.M., et al., Subclinical vitamin K deficiency in hemodialysis patients. Am J Kidney Dis, 2007. 49(3): p. 432-9.

26. Holden, R.M., et al., Vitamins $k$ and $d$ status in stages 3-5 chronic kidney disease. Clin J Am Soc Nephrol, 2010. 5(4): p. 590-7.

27. Schurgers, L.J., et al., Vitamin K-containing dietary supplements: comparison of synthetic vitamin K1 and natto-derived menaquinone-7. Blood, 2007. 109(8): p. 3279-83. 



\section{CHAPTER 8}

\section{General Discussion}

Ellen C.M. Cranenburg

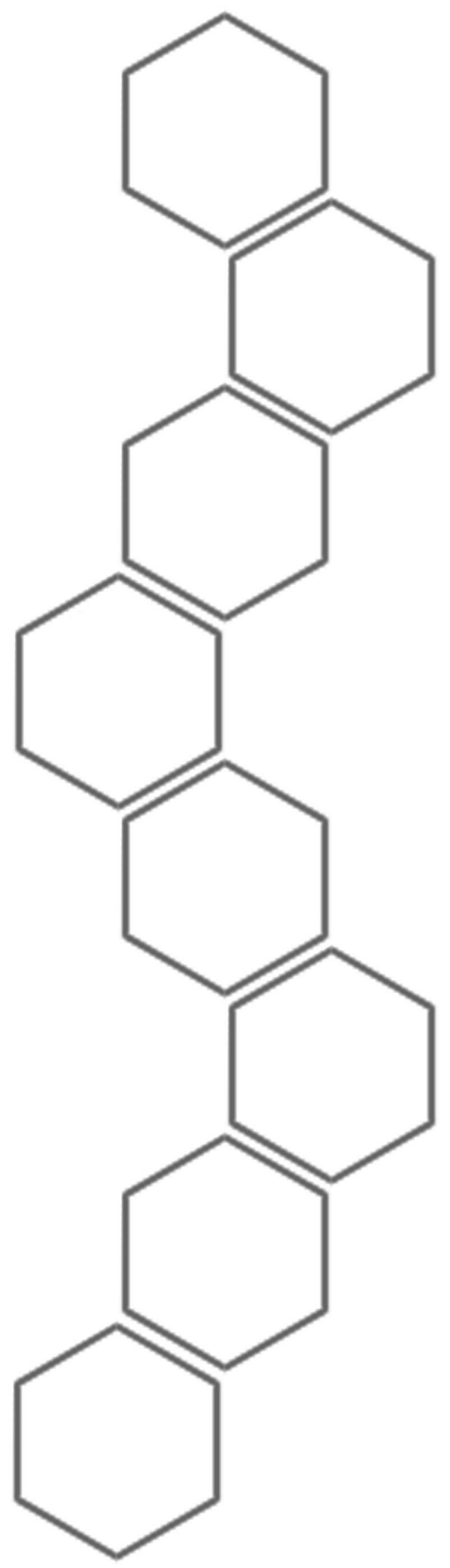

VitaK and Cardiovascular Research Institute Maastricht (CARIM), Maastricht University, Maastricht, The Netherlands 


\section{Introduction}

Matrix $y$-carboxyglutamate (Gla)-protein (MGP) is the most potent local inhibitor of arterial calcification currently known. The vascular calcification phenotype of MGP null mice could not be rescued by raising systemic MGP levels [1]. However, restoration of MGP expression in the vascular smooth muscle cells (VSMC), prevented the development of arterial calcification. These experiments show that MGP exerts its function as a calcification-inhibitor locally in its tissue of synthesis. Although MGP may not be active in the circulation, circulating MGP species may reflect the (inhibition of) calcification processes in the vascular wall.

MGP can undergo two post-translational modifications: $\boldsymbol{\gamma}$-carboxylation of specific glutamate (Glu) residues and phosphorylation of serine residues. The conversion of Glu residues into Gla residues confers calcium-binding properties to vitamin K-dependent proteins, also called Gla-proteins. $\gamma$-Carboxylation is thought to be essential for the function of these proteins [2]. Indeed, it was demonstrated in the MGP null mice that MGP required at least $4 \mathrm{Gla}$ residues to prevent extra-cellular matrix calcification [1]. In addition, the Gla-domain of MGP is involved in binding of bone morphogenetic protein-2 (BMP-2), an osteogenic growth factor [3]. As a result, binding of BMP-2 to its receptor is blocked, thereby inhibiting its action [4]. Next to binding of MGP to crystal nuclei to prevent their growth, this is considered a mechanism of action of MGP.

The exact role of phosphoserine residues remains to be elucidated, but it has been suggested that they play a role in the secretion of MGP into the extracellular matrix. We have hypothesized that these negatively-charged groups are also able to bind to insoluble calcium salts [5]. In addition, experiments with VSMC have shown that phosphorylated MGP residues may also contribute to its calcification-inhibitory activity [6]. As a result of both post-translational modifications, several MGP species may be synthesized: phosphorylated (pMGP), nonphosphorylated (desphospho, dpMGP), carboxylated (cMGP), or uncarboxylated (ucMGP). With the development of species-specific antibodies, it became possible to detect MGP species in tissues by immunohistochemical staining and in the circulation by enzyme-linked immunoassays (ELISA). 


\section{General Discussion}

Vitamin $\mathrm{K}$ serves as a cofactor for the enzyme $\mathrm{\gamma}$-glutamyl carboxylase, thereby promoting the carboxylation reaction that is coupled to vitamin $\mathrm{K}$ recycling. Vitamin $\mathrm{K}$ is a group name for several $\mathrm{K}$ vitamins, with vitamin $\mathrm{K}_{1}$ (phylloquinone) and vitamin $\mathrm{K}_{2}$ (menaquinones) being responsible for the dietary vitamin $\mathrm{K}$ intake. Vitamin $\mathrm{K}_{1}$ is present in green leafy vegetables and vitamin $\mathrm{K}_{2}$ in cheese, curd, and the Japanese food natto. The recommended daily allowance (RDA) is $1 \mu \mathrm{g} /$ day/kg bodyweight, which is based on requirements for normal haemostasis. The requirements for carboxylation of extra-hepatic Gla-proteins, including MGP, has not been taken into consideration in establishing the RDA [7].

Arterial calcification is associated with cardiovascular morbidity and mortality in the general population, as well as in high-risk patient populations [8-11]. These include patients with chronic kidney disease (CKD) [8, 12], diabetes mellitus (DM) [10], and inflammatory diseases including rheumatic diseases $[13,14]$. Arterial calcification is currently regarded as an active, cell-mediated process. The VSMC and their potential to secrete matrix vesicles that can form the nidus for calcification, and to transform from a contractile phenotype into osteoblastic/chondrocytic-phenotype, play a central role in this process [15]. The balance between promoters and inhibitors of calcification is thought to play an important role in the development and progression of arterial calcification [16]. A general literature overview on MGP, vitamin K, and arterial calcification is given in Chapter 1.

Circulating MGP levels may serve as a biomarker for prevalent arterial calcification both in high-risk patient populations and the general population. Measurements of MGP may also contribute to the identification of those patients at high risk to develop arterial calcification, due to an impaired MGP as a consequence of vascular vitamin K deficiency. Within this context, we developed ELISA to measure circulating MGP species and investigated their potential for research and clinical use. 


\section{Circulating MGP measurements}

\subsection{The mono-antibody MGP assay measuring t-ucMGP}

Before we started the work described in this thesis, there were two assays describedin the literature to measure circulating MGP species. One is a radio-immunoassay, not commercially available, that is based on MGP polyclonal antibodies purified from human bone [17]. The second assay is a single-antibody (competitive) enzyme-linked immunosorbent assay (ELISA), that is based on a monoclonal antibody raised against the non-phosphorylated MGP sequence 3-15 $[18,19]$. This assay therefore measures circulating levels of total desphospho MGP species (t-dpMGP) and does not reflect the carboxylation status of MGP.

In Chapter 2, we report on the development of a single-antibody ELISA for human circulating total ucMGP (t-ucMGP) species. Using a monoclonal ucMGP antibody (directed against the sequence 35-49) in immunohistochemical techniques, it was shown that ucMGP species accumulated at sites of arterial calcification in the vascular wall [20]. Circulating UCMGP species may therefore be particularly suited to reflect the presence of arterial calcification. The accumulation of ucMGP species at sites of arterial calcification and its probable high affinity for hydroxyapatite [20-22], suggest that ucMGP species are not easily set free into the circulation. We hypothesize therefore that in the presence of arterial calcification, the levels of circulating UCMGP species are decreased. The specificity of the t-ucMGP assay was demonstrated by using synthetic MGP peptides; only the ucMGP peptides reacted with the monoclonal ucMGP antibody, whereas no affinity was observed for cMGP peptides. Full length ucMGP was also identified by mass spectrometry as the major MGP species recognized by the monoclonal ucMGP antibody.

Circulating t-ucMGP levels were measured in coronary angioplasty, aortic valve stenosis, dialysis, and calciphylaxis patients. All patient populations had significantly lower t-ucMGP levels compared to healthy subjects of the same age. There wassome overlap between the t-ucMGP values of the coronary angioplasty and aortic stenosis patients, which may be explained by the expected larger inter-individual variation in the extent of vascular calcification in these patient populations. In addition, aortic stenosis patients can be free from atherosclerosis, whereas coronary angioplasty patients suffer from intimal (atherosclerotic) calcification. Almost all hemodialysis and calciphylaxis patients had lower t-ucMGP levels than the healthy subjects, indicating that t-ucMGP is a marker capable of discriminating between healthy subjects and patients with end-stage renal disease (ESRD; CKD stage V). These results support our hypothesis of low circulating t-ucMGP levels in the presence of arterial calcification and indicate that circulating t-ucMGP has the potential to become a non-invasive biomarker for cardiovascular calcification. 


\section{General Discussion}

However, one of the major limitations of this study was the absence of arterial calcification quantification. Nevertheless, we measured CAC in our second study to investigate the potential of circulating t-ucMGP as a biomarker for cardiovascular calcification.

Circulating t-ucMGP levels were measured in a population of hemodialysis (HD) patients, who underwent multislice CT scanning (MSCT) to assess the extent of coronary artery calcification (CAC), described in Chapter 3. In HD patients, CAC has been associated with the severity of coronary artery disease and the presence of stenotic lesions [23] as well with increased mortality [24]. This study enabled us to correlate t-ucMGP measurements with the extent of CAC in a high-risk patient population. Two reference populations were used for comparison of t-ucMGP levels: healthy subjects and patients with rheumatoid arthritis (RA) who had no CAC (CAC score 0 on MSCT scan). The healthy subjects did not underwent MSCT scanning. We therefore included the group of RA patients, although it was at that time unknown if RA itself influenced t-ucMGP levels. The HD patient cohort had significantly lower t-ucMGP levels compared to both the healthy subjects and the RA patients without CAC. CAC scores in the HD patients ranged from 0 (no CAC) to 2928 (extreme CAC). When the HD patients were divided into tertiles according to CAC score (CAC $\leq 103.0,103.1$ to 600.0 , and $\geq 600.1$ ), patients in the low CAC tertile had significantly higher t-ucMGP levels compared to the other tertiles. In addition, t-ucMGP levels were inversely correlated with CAC in the HD patients. This association persisted after correction for age, dialysis vintage, and hs-CRP in multivariate regression analysis. The maximal number of possible confounders which could be included in the regression analysis was four (including t-ucMGP), due to the limited number of patients $(n=40)$. However, with the variables age, dialysis vintage, and hs-CRP included, we could adjust at least for three of the most important potential confounders regarding CAC development in ESRD patients. This study confirms the finding that t-ucMGP levels are low in patient populations with a high burden of vascular calcification (HD and calciphylaxis patients). Moreover, t-ucMGP measurements may not only be used for inter-group comparisons of arterial calcification, but also for estimating the individual extent of CAC.

In cooperation with the Department of Cardiology, RWTH Aachen University, t-ucMGP levels were also measured in patients diagnosed with aortic valve stenosis [25]. $\mathrm{s}, \mathrm{t}-$ ucMGP levels in these patients were significantly lower compared to a control group, which is in accordance with our findings. Serum t-ucMGP did not correlate with either severity of aortic stenosis or extent of aortic valve calcification. 
To investigate the potential predictive value of circulating t-ucMGP for overall and cardiovascular mortality, we determined serum t-ucMGP levels in a prospective, well-defined Dutch cohort of 949 incident HD and peritoneal dialysis (PD) patients (NECOSAD; Chapter 4). Circulating t-ucMGP levels were measured in serum samples obtained at the time of 3 months of dialysis treatment. Almost all patients (>96\%) had circulating t-ucMGP levels below the normal range. PD patients had significantly higher t-ucMGP levels compared to HD patients, but these levels were still well below those of healthy subjects. When patients were divided into tertiles of t-ucMGP levels, no difference was found between high, medium, and low circulating t-ucMGP in predicting mortality risk. The circulating low t-ucMGP levels are in accordance with our previous results. This study demonstrated that patients who started recently with dialysis treatment ( 3 months) already have low t-ucMGP levels. This may be explained by the fact that all patients have reached the final stadium of chronic kidney disease (ESRD).

Circulating t-ucMGP levels were shown to inversely correlate with glomerular filtration rate (GFR) in patients with stable cardiovascular disease (CVD) [26]. Moreover, in patients with aortic valve disease, those with CKD stage 4 had significantly lower t-ucMGP levels than patients with normal to moderately impaired renal function (CKD stage 1-3) [25]. It is therefore likely that circulating t-ucMGP levels are already lowered in the previous stages of CKD.

This was the first study investigating t-ucMGP levels in a large group of PD patients. These patients also had low t-ucMGP levels. On average, HD patients had even lower t-ucMGP levels, but the difference was relatively small. It has been found in the NECOSAD cohort that patients with moderate to severe aortic arch calcifications were more often treated with HD than PD[27]. From the patients without calcification, the majority (55\%) underwent PD treatment. The higher t-ucMGP levels in PD patients might therefore be explained by less (severe) arterial calcification found in these patients. Residual confounding may be another explanation of the found difference in t-ucMGP levels between PD and HD patients. 


\section{General Discussion}

There was no difference in t-ucMGP levels between patients with and without CVD. This might be due to the fact that the range of t-ucMGP levels in CVD patients is relatively wide (Chapter 2; coronary angioplasty and aortic stenosis patients). The presence of ESRD seemed to result in a more extensive lowering of t-ucMGP levels than the presence of CVD (Chapter 2). In the NECOSAD cohort, in which virtually all patients had very low $\mathrm{t}$ ucMGP levels, the effect of CVD on t-ucMGP levels might therefore not have been detected. Circulating t-ucMGP correlated inversely with pulse pressure in the total patient population; this association persisted after correction for several variables, including age, hsCRP, calcium, and phosphate levels. This is in agreement with our findings of an inverse correlation between t-ucMGP and CAC in HDpatients (Chapter 3). These results support the hypothesis that t-ucMGP may serve as a biomarker for prevalent arterial calcification.

The mortality data in the NECOSAD study relied on observations of treating physicians, which could induce some error in the definition of the exact cause of death. The classification of deaths with an uncertain or missing cause as CV mortality did influence our results, as previously described for PD patients.

The major limitation of this study was that no sensitive imaging technique such as MSCT scanning was applied to evaluate the precise amount of arterial calcification in the current population. The relationship between t-ucMGP, the extent of vascular calcifications, and mortality could therefore not be evaluated in the present study. However, this was the first study in which circulating t-ucMGP levels were determined in a large number of incident HD as well as PD patients.

The extent of aortic calcification was investigated in 384 patients of the NECOSAD cohort[27]. This was done by screening annual chest X-rays for calcification in the aortic arch and patients were categorised as having no, moderate, or severe calcification. The progression of aortic calcification was significantly related to an increased mortality risk. Together, we investigated the association between t-ucMGP levels (measured at 3 months of dialysis) and aortic calcification in the NECOSAD cohort. Although patients without aortic calcification had somewhat higher t-ucMGP levels $(191 \pm 87 \mathrm{nmol} / \mathrm{L} ; \mathrm{n}=83$ ) compared to patients with moderate $(176 \pm 69 \mathrm{nmol} / \mathrm{L} ; \mathrm{n}=205)$ and severe calcification $(172 \pm 62 \mathrm{nmol} / \mathrm{L} ; \mathrm{n}=96)$, the difference was not statistically significant. It is possible that the screening of $X$-rays is too insensitive to detect small arterial calcifications, resulting in misclassification of patients as having no aortic calcification. 


\subsection{The dual-antibody assays measuring dp-ucMGP and dp-cMGP}

Since MGP carboxylation is vitamin K-dependent, certain species may also reflect the availability of vitamin $\mathrm{K}$ present in the vascular wall. Clinically, an insufficient vascular vitamin $\mathrm{K}$ status may result in an increased risk for arterial calcification. We aimed to develop MGP assays which would reflect the availability of vitamin $\mathrm{K}$ in the vascular wall for MGP carboxylation (vascular vitamin K status).

We developed two assays detecting circulating desphospho-uncarboxylated (dp-uc) MGP and desphospho-carboxylated (dp-c) MGP. Due to the absence of phosphoserine residues, we hypothesized that these MGP species may be more easily set free into the circulation than the corresponding phosphorylated species, independent of the presence of calcification in the vascular wall. They may therefore mainly be influenced by the availability of vitamin $\mathrm{K}$ (see also paragraph 2.3). Changes in systemic vitamin $\mathrm{K}$ status, for instance resulting from the use of vitamin $\mathrm{K}$ supplementation or vitamin $\mathrm{K}$ antagonists (coumarin derivatives), should then be reflected in circulating MGP measurements.

This was tested in the study described in Chapter 5, which revealed that particularly dpucMGP is sensitive to changes in vitamin $\mathrm{K}$ status. Healthy subjects receiving vitamin $\mathrm{K}$ supplementation during several weeks had significantly decreased dp-ucMGP levels, whereas subjects who received vitamin $\mathrm{K}$ antagonists (acenocoumarol) during 4 weeks had significantly increased dp-ucMGP levels. The dp-cMGP levels also decreased after highdosage vitamin $\mathrm{K}$ supplementation (45 mg MK-4 during 6 months), it is currently not known why (see also paragraph 2.3). Treatment with acenocoumarol had no significant effect on dp-cMGP levels. Circulating dp-cMGP seems therefore less suited as a marker for vitamin $\mathrm{K}$ status. The use of a ratio of uncarboxylated and carboxylated MGP may not be useful to assess vascular vitamin $K$ status, since the ratio will not change upon longterm vitamin $\mathrm{K}$ supplementation as both dp-ucMGP and dp-cMGP levels will decrease.

Since a direct comparison between the commercially available MGP assay (t-dpMGP assay) and the assays developed by our group (t-ucMGP, dp-ucMGP, and dp-cMGP assay) had not yet been performed, we also measured MGP levels in several patient populations with these assays. The patient populations included patients with aortic valve disease (AVD) and ESRD, in which the prognostic value of cardiovascular calcification has been clearly established $[8,12,28]$. Additionally, a rheumatic disease (RD) patient population consisting of patients with systemic lupus erythematosus (SLE), rheumatoid arthritis (RA), chondrocalcinosis (CC), gout, and diffuse idiopathic skeletal hyperostosis (DISH) was included. 


\section{General Discussion}

Overall, patients with RD had significantly higher dp-ucMGP levels and significantly lower t-ucMGP levels. In the RD patient groups (SLE, RA, CC, gout, and DISH), these results were seen in patients with gout andCC, respectively. However, the differences were very small and the influence of cartilage disease on circulating MGP levels seems to be minimal compared to that of CVD. Patients with SLE and RA did not have significantly different circulating MGP levels, indicating that a poor vascular vitamin $\mathrm{K}$ status might not contribute to the increased arterial calcification risk in these patients.

Patients with ESRD and AVD had very high circulating dp-ucMGP levels, indicating a poor vascular vitamin $\mathrm{K}$ status. In a preliminary report, the group of Massy in cooperation with VitaK described dp-ucMGP measurements in 107 patients with different stages of chronic kidney disease (CKD stage 2-5D) [29]. It was found that circulating dp-ucMGP levels increased progressively with CKD stage. The use of coumarins was also independently associated with plasma dp-ucMGP levels.

Moreover, dp-ucMGP was positively and independently associated with the severity of aortic calcification. In a crude analysis, a plasma dp-ucMGP level above the median level (>921 pmol/L) predicted overall mortality (log rank test $p=0.006)$. Ueland et al. from the Rikshospitalet Radiumhospitalet Medical Centre, University of Oslo, investigated dpucMGP and dp-cMGP levels in 147 patient with calcific valvular aortic stenosis (submitted for publication). Patients with high levels of dp-ucMGP ( $>950 \mathrm{pmol} / \mathrm{L}$ ) had an increased all-cause mortality risk compared to patient with low dp-ucMGP levels (HR 9.2, p<0.001). For dp-cMGP, there were no significant associations with mortality. In this study there was also a positive correlation between dp-ucMGP and warfarin use $(r=0.65, p<0.001)$. A poor vascular vitamin $\mathrm{K}$ status, indicated by high dp-ucMGP levels, may therefore be associated with an increased mortality. Importantly, in both of these studies the dp-ucMGP levels of the patients were significantly higher compared to normal controls, which is in agreement with our findings in ESRD and AVD patients.

The dp-cMGP levels in AVD and ESRD patients were 1.4 and 2.6 times fold higher than those in the corresponding age-groups, respectively. The t-ucMGP levels were significantly lower in these patient populations, which is in accordance with the results described in Chapter 2. The t-dpMGP levels were significantly higher in AVD patients; otherwise there were no significant differences between patient populations and the healthy reference groups of comparable age. In the past, low as well as high t-dpMGP levels have been reported in patients with atherosclerotic disease $[18,19]$. Circulating t-dpMGP levels increased significantly after MK-7 supplementation, after coumarin treatment and also in the placebo group. 
The inconsistency of t-dpMGP results, together with the large overlap between patient and reference values, indicates that this assay is not capable of identifying patient populations and would not be suited for individual patient diagnosis.

An important finding was that dp-ucMGP and dp-cMGP levels could not be measured in serum. We hypothesized that the thrombin generated during serum preparation could be the cause of this. Previous experiments with incubation of full-length synthetic MGP with thrombin, a circulating serine-protease, resulted in four MGP fragments as analyzed with mass-spectrometry (T. Hackeng, personal communication). MGP therefore appears to have several cleavage sites for thrombin, and might be degraded by the thrombin, resulting in non-measurable serum dp-ucMGP and dp-cMGP levels. More research is needed to confirm this hypothesis.

The measurement of circulating dp-ucMGP confirms the previous finding of uncarboxylated, inactive MGP species in the circulation of healthy subjects, measured with the tucMGP assay (Chapter 2). This has also been reported for osteocalcin, a vitamin Kdependent protein synthesized in bone, of which uncarboxylated levels are also present in the circulation of healthy subjects. The decrease of dp-ucMGP levels to almost immeasurably low levels after 6 months of VKS, indicates that the even in healthy subjects the vascular vitamin $\mathrm{K}$ status might be insufficient for maximal MGP carboxylation. Based on our results, each assay may have its own application for research and/or for diagnostic use. From these assays, the dp-ucMGP assay seems to be particularly suited to assess vascular vitamin $\mathrm{K}$ status. 


\subsection{Hypothesis regarding circulating MGP species}

In Figure 1, our general hypothesis regarding circulating MGP species and vitamin Kdependent regulation of vascular calcification is illustrated. This hypothesis does not explain the results obtained with the dp-cMGP assay. Since carboxylation of MGP is vitamin K-dependent, we expected that dp-cMGP levels would increase upon high intake of vita$\min \mathrm{K}$ (use of vitamin $\mathrm{K}$ supplementation) and decrease upon vitamin $\mathrm{K}$ deficiency (use of vitamin $\mathrm{K}$ antagonists). Our expectation was that the dp-cMGP species would respond to changes in vitamin $\mathrm{K}$ status in an opposite direction as the dp-ucMGP species. However, circulating dp-cMGP levels decreased upon high-dosage vitamin $\mathrm{K}$ supplementation and remained unchanged upon (short-term) use of vitamin $\mathrm{K}$ antagonists (Chapter 5 ). One possible explanation for these results may be found in the second post-translational modification: phosphorylation. It is possible that upon (high-dosage) vitamin Ksupplementation, dp-cMGP species are not only better carboxylated but that the carboxylated precursor proteins are also better substrates to be phosphorylated. This would result in formation of $\mathrm{p}$-cMGP species, which remain undetected with the dp-cMGP assay. The dp-cMGP levels would thus be decreased. Another explanation could be that the dpcMGP species that are synthesized upon vitamin $\mathrm{K}$ supplementation remain in the tissue and are not readily secreted into the circulation. It is clear that more fundamental research into MGP phosphorylation is necessary to investigate this hypothesis and to investigate why dp-cMGP species do not respond to lower vitamin $\mathrm{K}$ dosages and to short-term vitamin $\mathrm{K}$ antagonist use. 
Concerning the binding of MGP to areas of calcification in tissue, we expect that the negatively-charged phosphoserine residues contribute significantly to the calcium-binding capacity of MGP. The binding of UcMGP species to areas of calcification in tissue, as demonstrated with immunohistochemical staining, is most probably due to the presence of phosphoserine residues. The role of phosphoserine residues has been examined in a cell model of calcification [6]. This model consisted of VSMC exposed to high extracellular calcium concentrations. The synthetic MGP peptides $\mathrm{PMGP}^{3-15}$ and dpMGP ${ }^{3-15}$, contain three serine residues that are phosphorylated or non-phosphorylated, respectively. These peptides do not contain Glaresidues. After addition of the (biotinylated) peptides to the calcifying VMSC, staining revealed that $\mathrm{PMGP}^{3-15}$ specifically adhered to vesicular structures derived from the VSMC. These vesicles were presumably matrix vesicles, preceding the formation of calcification [30]. In contrast, $\mathrm{dpMGP}^{3-15}$ did not bind to either the cell surface or the vesicles. pMGP $\mathrm{P}^{3-15}$ was also able to inhibit the calcification process, whereas dpMGP ${ }^{3-15}$ had no measurable effect. These results indicate that MGP containing phosphoserine residues, but no Gla residues, is able to bind to (initial) sites of calcification.

The presence of Gla-residues does not seem to be a prerequisite for MGP's binding to calciumsalts and/or -crystals. Indeed, Price et al. had showed previously with in vitro experiments that thermal decarboxylation of MGP did not affect its calcium-binding properties [21]. The majority of the uncarboxylated MGP species measured with the t-ucMGP assay may therefore be phosphorylated ( $\mathrm{p}$-ucMGP), whereas only a minor part may be nonphosphorylated (dp-ucMGP). The t-ucMGP levels would therefore behave as the $p$-ucMGP levels indicated in Figure 1D. The phosphorylated, uncarboxylated species will adhere to the sites of calcification (low circulating t-ucMGP levels) whereas non-phosphorylated, uncarboxylated MGP species will be easily set free in the circulation (high dp-ucMGP levels). Taken also into account that dp-ucMGP species may constitute only a minor part of the total uncarboxylated MGP species, this may result in a combination of high circulating t-ucMGP levels and low dp-ucMGP levels in certain patient populations. Since phosphorylation is often associated with cellular secretion of proteins, it is at least feasible that in the diseased vessel MGP phosphorylation is hampered, leading to decreased circulating levels. This could be an alternative explanation for the low circulating t-ucMGP levels in patients with vascular calcifications. Other alternative explanations may be lower synthesis of MGP and increased degradation of MGP before carboxylation in these patients. 


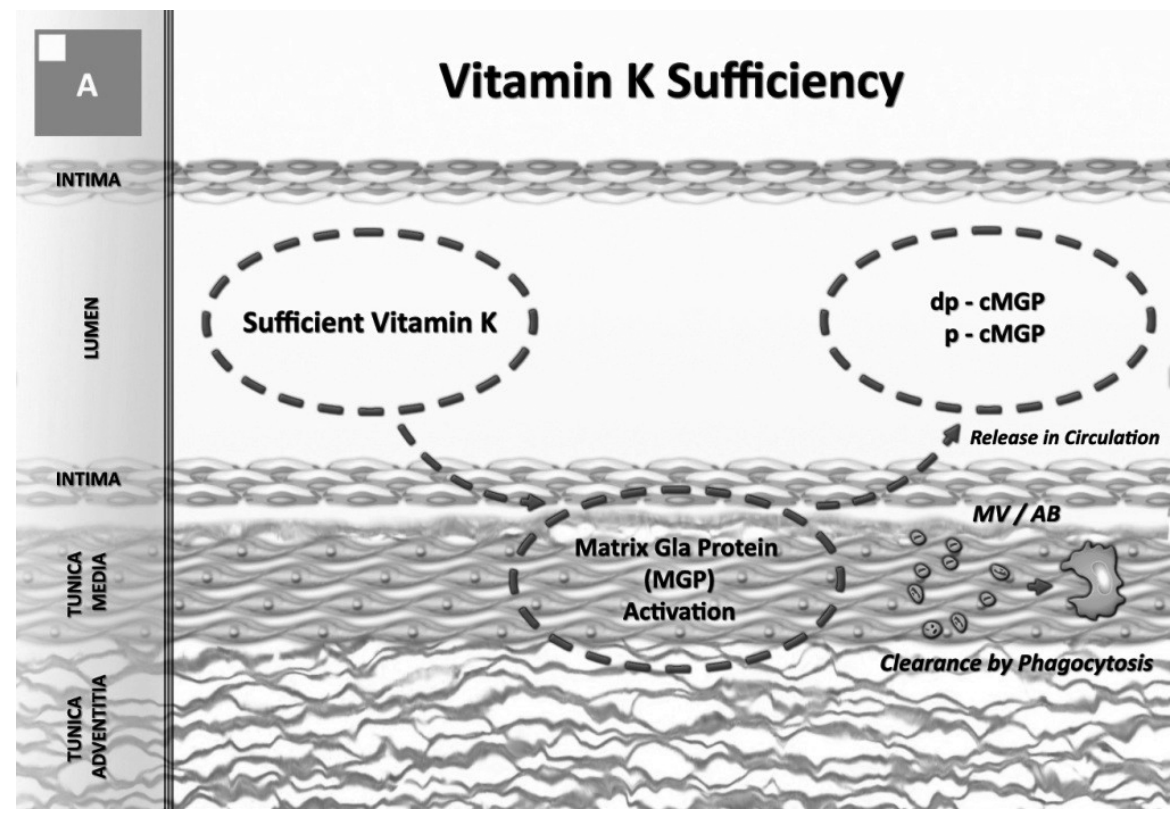

\section{A. Vitamin K sufficiency}

In the case of sufficient vitamin $\mathrm{K}$ supply (via dietary intake) and in the absence of vascular disease, all MGP species synthesized in the VSMC are activated to prevent vascular calcification. Macrophages and/or surrounding VSMC will clear matrix vesicles and apoptotic bodies (MV/AB) that have the potential to form the nidus for calcification through phagocytosis. The nidus for calcification is then absent. There is no formation of hydroxyapatite in the tunica media, to which MGP can bind. MGP species that are set free into the circulation are desphospho-carboxylated (dp-c) and phospho-carboxylated (p-c) MGP. 


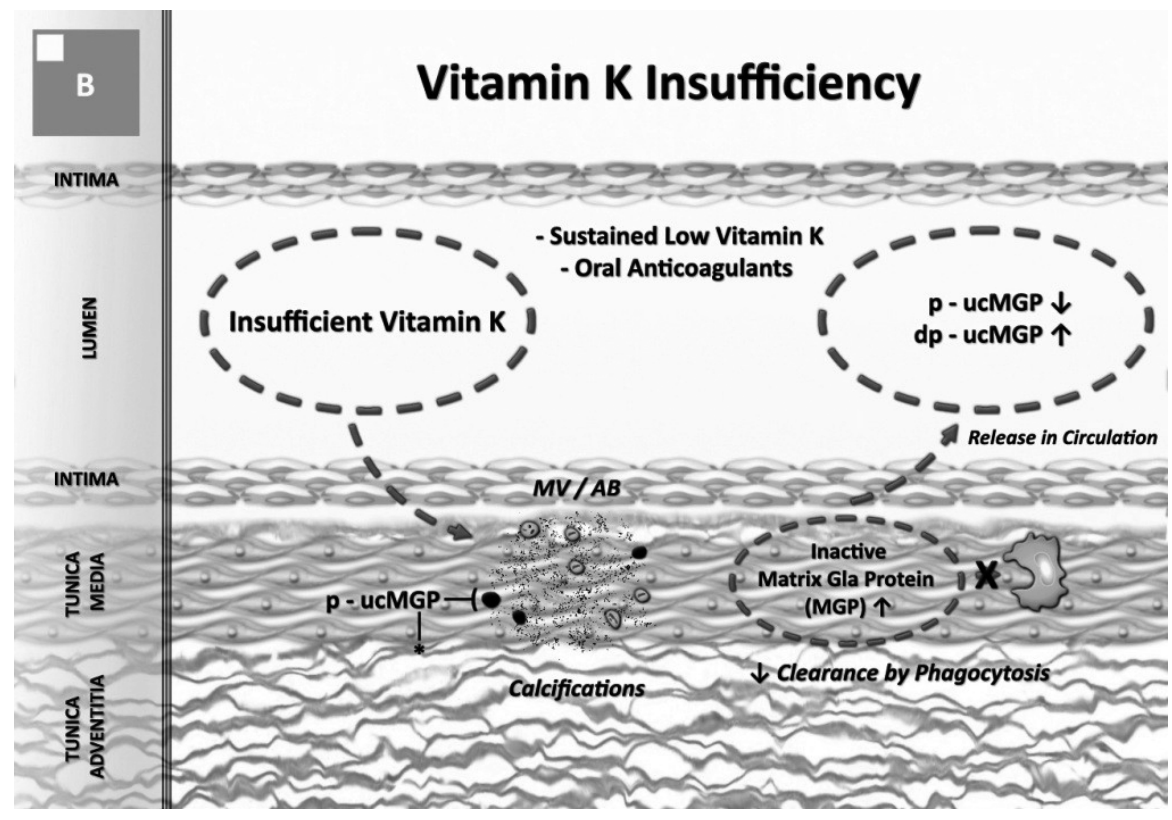

\section{B. Vitamin K insufficiency}

Vitamin $\mathrm{K}$ insufficiency results from a low dietary vitamin $\mathrm{K}$ intake or the use of vitamin $\mathrm{K}$ antagonists (coumarins). The expression of MGP is normal. Uncarboxylated, inactive MGP will be synthesized, possibly leading to decreased clearance of the calcification vesicles. This will result in deposition of negatively charged phospholipid-remnants in the arterial media. These remnants have the capacity to nucleate calcium and phosphate, and subsequently calcify in the absence of the calcification-inhibitory function of MGP. The phosphorylateduncarboxylated ( $p-u c$ ) MGP species will bind to vascular calcifications, and thus p-ucMGP in the circulation is lowered. The dp-ucMGP species are easily set free into the circulation, since there are no calcium-binding groups present in these proteins, resulting in increased levels in the circulation. In the majority of healthy subjects (and patients) the vitamin K status seems to be insufficient for MGP carboxylation, as deduced from inactive ucMGP species in the circulation. 


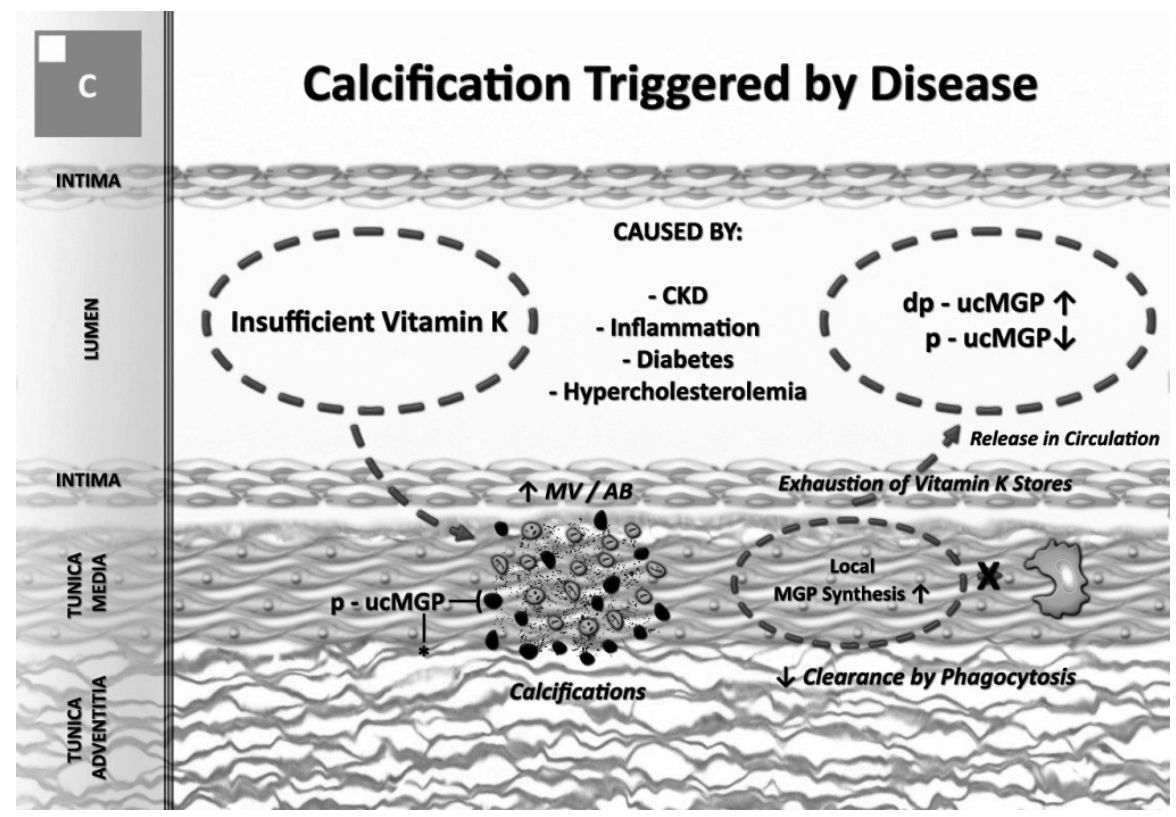

C. Vascular calcification present

In diseases leading to the shedding of high numbers calcification-vesicles, the development of calcification is triggered and the need for active MGP is high, to counteract the nidus for calcification. This includes diseases with a disordered mineral balance (high calcium-phosphate product) and inflammation (leading to apoptosis). Therefore, the demand for vitamin $\mathrm{K}$ is increased. The average dietary vitamin $\mathrm{K}$ intake is insufficient to address this higher demand of vitamin $\mathrm{K}$ required for the activation of all newly synthesized MGP. Therefore, a major part will be synthesized as UcMGP species, unable to stop the calcification process. The ucMGP species with phosphoserine residues ( $p$-ucMGP) will bind to local vascular calcifications, which will be measured by the lower plasma p-ucMGP levels. 


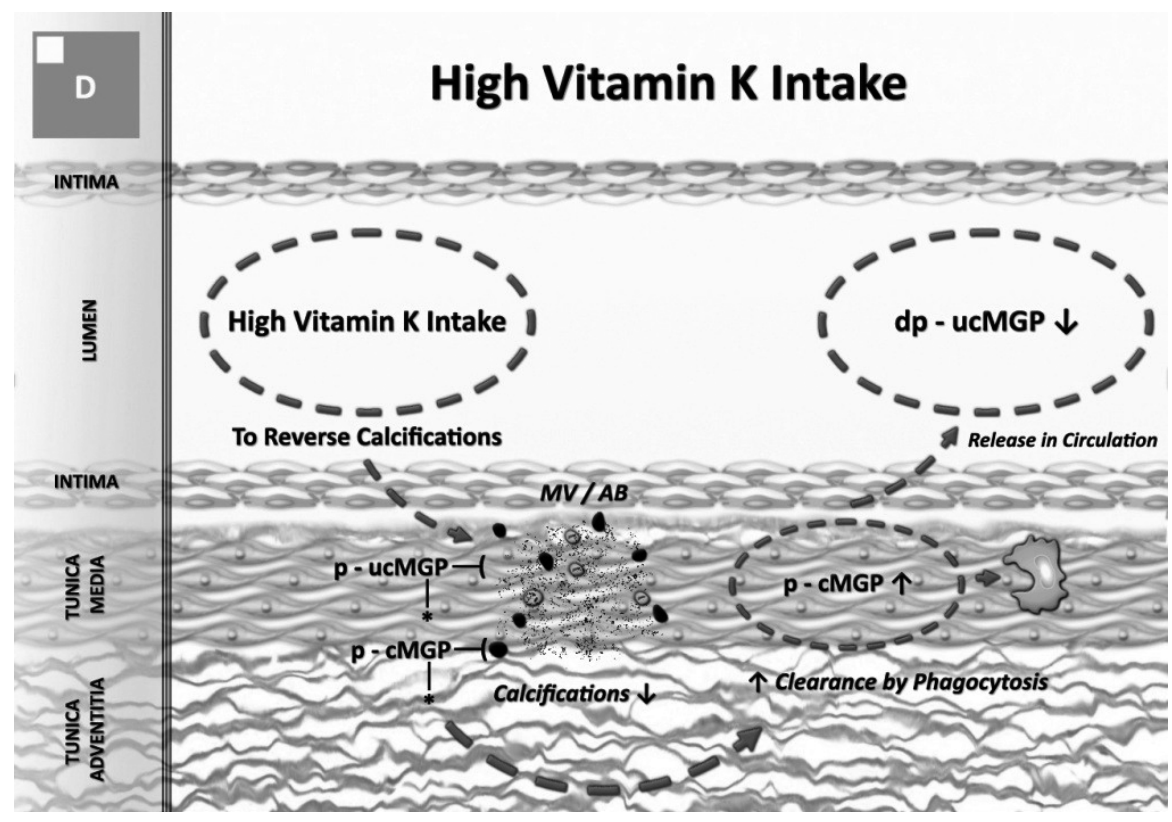

D. Increasing the vitamin $\mathrm{K}$ intake (high vitamin $\mathrm{K}$ intake)

When increasing the vitamin $\mathrm{K}$ intake, all newly synthesized MGP will be activated via the $\gamma$-carboxylation reaction. This will result in synthesis of dp-cMGP and p-cMGP species. However, since we found that dp-cMGP levels decreased rather than increased upon vitamin $\mathrm{K}$ supplementation, it is possible that dp-cMGP is converted to $p$ cMGP, thereby decreasing the dp-cMGP levels (see paragraph 2.3.). As the active MGP fraction will support clearance/regression of calcification, the amount of calcification will reduce. In the circulation, this will be reflected by a lowered dp-ucMGP level, demonstrating a beneficial shift in the tissue. 


\section{Keutel syndrome investigations}

Keutel syndrome (KS) is a rare autosomal recessive disorder resulting from mutations in the MGP gene. We performed a study in two patients with KS, which included a characterization of the KS phenotype to further elucidate the function of MGP, as well as investigation of the presence of MGP in the circulation and tissues of KS patients (Chapter 6).

The first patient presented with exertional dyspnoea at the Department of Otorhinolaryngology of the University Medical Center Groningen. Laryngotracheoscopy revealed cricoid stenosis and an abnormal trachea, and subsequent CT scanning showed abnormal cartilage calcification. Cartilage calcification is the hallmark of KS and other clinical features, including brachytelephalangism and peripheral artery stenoses, were consistent with the diagnosis of KS [31]. Mutation analysis revealed a novel MGP mutation (c.61+1G>A), which is the fifth MGP mutation identified thus far. This particular mutation results in the loss of the consensus donor splice site at the exon1-intron1 junction. The previously identified MGP mutations were two splice site mutations and two mutations introducing a stop codon. Although all these mutations are expected to coincide with absent or non-functional MGP, circulating MGP species have not been characterized previously in KS patients.

The development of our species-specific MGP assays allowed us to investigate the presence of different circulating MGP species in the KS patient described above. We used the dpucMGP and dp-cMGP assays to characterize circulating MGP species. In addition, we used a very recently developed mono-antibody assay to measure phosphorylated MGP species (tpMGP). The patient had low dp-ucMGP levels, which were comparable to those of his firstdegree relatives, one of whom was a younger sibling without the MGP mutation (noncarrier). However, he had the lowest dp-cMGP levels and, surprisingly, the highest t-pMGP levels, compared to his relatives and healthy subjects. The high level of t-pMGP may be explained by a relatively high synthesis of MGP, possibly induced by the high calcification load.

Vitamin $\mathrm{K}$ supplementation was shown to decrease dp-ucMGP levels in healthy subjects, probably indicating an improved vascular vitamin $\mathrm{K}$ status and improved calcification inhibition by MGP (Chapter 6 ). We therefore aimed to assess whether vitamin $\mathrm{K}$ also affects MGP levels in our patient. If so, this would be a first indication that a KS patient may benefit from vitamin $\mathrm{K}$ supplements. Further exploration of the effect on clinical symptoms would then be justified. The patient received a high dose of $10 \mathrm{mg}$ vitamin $K_{1}$, which is the dosage and type of vitamin $\mathrm{K}$ that can be prescribed as a medical drug in the Netherlands (Konakion, Roche Nederland, Woerden, the Netherlands). 
No clinically relevant changes in either ucMGP or cMGP levels were seen after 3 months of supplementation. Probably, carboxylation of MGP was not possible due to the mutation, since only the first 14 amino-acids of the mutated protein are comparable with those of the mature MGP protein. In addition, dp-ucMGP and dp-cMGP levels were low in our patient, and therefore a modest effect due to vitamin $\mathrm{K}$ supplementation may not have been detected. Since the increased availability of vitamin K did not measurably affect MGP levels, it is unlikely that vitamin $\mathrm{K}$ supplementation would improve theclinical symptoms or the long-term outcome of this particular KS patient. MGP is a local inhibitor of calcification in the vascular wall. Increasing the circulating MGP level in the MGP null mice did not rescue the vascular calcification phenotype of these mice. The finding of MGP in the circulation of our KS patient is therefore not clear proof that MGP still has calcification-inhibitory activity. However, it does confirm that MGP is synthesized in the tissues of our patient.

Vascular evaluation of the patient, including MSCT scanning of the coronary arteries, revealed no signs of arterial calcification. This is consistent with a report by Hur et al., who describe that body CT scan of a KS patient (8 years) revealed cartilage calcifications, without involvement of the soft tissue [31]. KS patients seem to survive well into adulthood, and extensive vascular calcification has been reported only in one patient (see below). It should be noted, however, that vascular evaluation has not been performed in the majority of patients described in the literature [31-47]. Overall, the phenotype of KS patients, mainly characterized by cartilage calcification, seems to be in contrast with the arterial calcification phenotype of the MGP null mice.

There is one paper describing extensive arterial calcification in a KS patient. This paper describes the post-mortem examination of the first patient described by Keutel et al. in 1971. This examination revealed concentric arterial calcification in several vascular beds, including the coronary, hepatic, renal, meningeal, and cerebral arteries [48]. This is to our knowledge the only described post-mortem examination of a KS patient. Some tissues of this patient were available to examine the presence MGP species and other calcification inhibitors. Using the species-specific MGP antibodies, the presence of MGP - predominantly in its phosphorylated form - was shown in the vasculature. Elastic fiber calcification was seen in the skin. This is in accordance with a report from Nanda et al., who demonstrated fragmentation of mid-dermal elastic fibres in biopsies of normal-looking skin from two KS patients. These results suggest a possible role for MGP in preventing elastic fiber calcification in the skin (see also paragraph 5.1.). Unfortunately, no tissues were available from our Dutch KS patient. 


\section{General Discussion}

The difference in the arterial phenotype between the MGP null mice model and the human $\mathrm{KS}$ is intriguing. The apparent absence of arterial calcifications in our patient may be due to an insufficient sensitivity of the MSCT scanning. It has been shown in vascular tissue from young dialysis patients that apoptosis and damage of vascular smooth muscle cells is accompanied by increased tissue levels of ucMGP. This preceded clinically overt calcification and even positive Von Kossa tissue staining for calcification [49]. Irrespective of this, the KS cases described so far may represent mutations accompanied with some residual calcification-inhibiting activity of MGP, which might be sufficient to prevent the development of arterial calcification. In both the patients presented here we found strong indications of the presence of phosphorylated MGP. Even if this is not the entire mature MGP protein, but fragments of MGP containing phosphoserine residues, binding of calcium crystals by MGP might still be possible.

It may be hypothesized that other calcification inhibitors are upregulated in our patient that might prevent the development of arterial calcification. Our study is the first to confirm Kaartinen et al.'s hypothesis that KS patients lacking functional MGP have arteries containing mineral-inhibiting osteopontin, possibly allowing for the extended survival of KS patients compared to MGP null mice [50]. Patients with KS may not develop overt arterial calcification unless other risk factors are present: either the loss of another inhibitor or the presence of a promoter of arterial calcification. These initiators might be absent in the relatively young KS patients described in the literature so far (mean age 8.3 years), which is supported by the absence of laboratory abnormalities in $19 \mathrm{KS}$ patients. In contrast, several potential triggers may be present in the only KS patient with extensive arterial calcifications described so far (chronic bronchitis, chemotherapy for recurrent malignancy) [48].

Phosphorylated MGP was found to be present in both patients, indicating that some residual MGP activity might be present. Obviously, further investigation of the function of MGP phosphorylation is needed. Vitamin K supplements did not affect MGP activity in our patient, but might have a beneficial effect for patients in whom carboxylation of MGP is possible. The investigation of KS patients' phenotype and their circulating MGP species contributes to the understanding of MGP functions in various tissues, and helps to elucidate the differences in arterial phenotype between MGP-deficient mice and humans. 


\section{Vitamin $\mathrm{K}$ and vascular health}

\subsection{Vitamin $\mathrm{K}$ intake}

Low dietary vitamin $\mathrm{K}$ intake may be a risk factor for arterial calcification, probably due to decreased activity of MGP. The population-based Rotterdam study was the first to show an inverse correlation between dietary vitamin $\mathrm{K}_{2}$ intake (and not vitamin $\mathrm{K}_{1}$ intake) with cardiovascular calcification and death in men and women aged 55 years and over [51]. The correlation between vitamin $\mathrm{K}_{2}$ intake and CVD was further investigated in three observational studies conducted in Dutch female participants (aged 49-70 years) of the PROSPECT study. The first study among 1,689 women showed no association between dietary vitamin $\mathrm{K}_{1}$ and $\mathrm{K}_{2}$ intake and calcification of breast arteries. In the second study among 16,057 women, it was found that vitamin $\mathrm{K}_{2}$ intake (especially MK-7, MK-8, and MK-9 intake) was associated with a reduced risk for coronary heart disease (CHD) (HR 0.91, 95\% Cl 0.85-1.00) [52]. A third study among post-menopausal participants showed that a high dietary vitamin $\mathrm{K}_{2}$ intake was associated with reduced coronary calcification [53]. In these latter two studies, vitamin $\mathrm{K}_{1}$ intake was not related to the described outcomes.

The majority of observational studies investigating only vitamin $K_{1}$ intake have not shown an association between vitamin $K_{1}$ intake and cardiovascular outcomes. Jie et al. found in women aged $60-69$ years that those with abdominal aortic calcific lesions $(n=34)$ had a lower vitamin $K_{1}$ intake than those without these lesions $(n=79)$ [54]. This association was not confirmed in women aged between 70 and 79 years. In a cohort of 807 men and women aged 39-45 years without known CHD, Villines et al. found no significant correlation between vitamin $\mathrm{K}_{1}$ intake and CAC [55]. In 72,874 female nurses aged 38-65 years of the Nurses' Health Study (1984-2000), it was concluded that high vitamin $\mathrm{K}_{1}$ intake $(300 \mu \mathrm{g} /$ day) is a marker for slightly reduced risks of total and non-fatal CHD. These associations were independent of established CVD risk factors, but were attenuated after adjustment for dietary variables associated with CHD (for instance fat, cereal fiber, and folate). The authors hypothesized that dietary and lifestyle patterns associated with $\mathrm{K}_{1}$ intakes, rather than the intake of the nutrient itself, may account for all or part of the weak association. In a second study by Erkkila et al. in 40,087 men aged 40-75 years of the Health Professionals' Follow-up Study (1986-2000), no significant associations were found between vitamin $\mathrm{K}_{1}$ intake and $\mathrm{CHD}$ events and strokes after adjustment for lifestyle and dietary factors. When controlling for lifestyle and other dietary factors associated with CVD in statistical analysis, the association between vitamin $\mathrm{K}_{1}$ intake and CVD is often lost. The main source of dietary vitamin $K_{1}$ is formed by green leafy vegetables, which indicate generally healthier diets and lifestyle $[53,56]$. Indeed, a strong overlap was found between low vitamin $\mathrm{K}_{1}$ intake and other dietary CVD risk factors in the Framingham Offspring Cohort [57]. Vitamin $\mathrm{K}_{1}$ can therefore be seen as an intake marker for a heart-healthy diet [53]. 


\section{General Discussion}

HD patients are at high risk to develop arterial calcification [8, 12]. Based on a typical renal diet, it can be predicted that the vitamin $\mathrm{K}$ intake of HD patients is low $(80 \mu \mathrm{g}$ or less of vitamin $\mathrm{K}$ per day) [58]. Booth et al. reported low circulating $\mathrm{K}_{1}$ levels and a poor carboxylation grade of osteocalcin (OC) in $29 \%$ and $93 \%$, respectively, of a cohort of $142 \mathrm{HD}$ patients [59]. A recent study by the same group in 172 subjects with stage 3 to 5 CKD, showed that the criteria for subclinical vitamin $\mathrm{K}$ deficiency were met by $6 \%$ of the patients based on circulating $\mathrm{K}_{1}$ measurements, $60 \%$ based on the carboxylation grade of $\mathrm{OC}$, and $97 \%$ based on protein induced by vitamin $\mathrm{K}$ absence II levels (PIVKA-II; uncarboxylated prothrombin) [60]. Due to its lipophilic properties and incorporation into lipoproteins, vitamin $\mathrm{K}$ is not expected to be removed by HD [61]. It can therefore be hypothesized that the vitamin $\mathrm{K}$ deficiency seen in HD patients may be due to a diminished dietary intake, which may increase the risk of arterial calcification. We have therefore investigated vitamin $\mathrm{K}$ intake as well as vitamin $\mathrm{K}$ status in a HD patient population (Chapter 7 ). We found a relatively low vitamin $\mathrm{K}$ intake in $40 \mathrm{HD}$ patients compared to intakes previously found in the Netherlands ( $>225 \mu \mathrm{g} /$ day) [51-54, 61, 62]. The second important finding was that both hepatic as well as extra-hepatic vitamin $\mathrm{K}$ status were strikingly poor, with the most extreme situation for the vascular wall. Low vitamin $\mathrm{K}$ intake of HD patients may be related to the dietary regimen generally prescribed for HD patients, which includes restriction of sodium and potassium intake [63]. Vitamin $\mathrm{K}$ intake was lower on weekend days and dialysis days as compared to week days, reflecting a different dietary pattern on these days.

Vitamin $\mathrm{K}$ status was investigated using measurements of circulating vitamin K, PIVKA-II, dp-ucMGP, and OC. Circulating vitamin $K$ levels were below the normal range in almost half of the patients. The majority of the menaquinones (MK-5 through MK-13) were below the lower detection limit, which is usually found in humans [7]. The high PIVKA-II levels found in the vast majority of patients indicate that vitamin $\mathrm{K}$ availability in the liver is too low to produce fully carboxylated clotting factors. The high dp-ucMGP levels found in all patients confirm our previous findings in HD patients and indicate a poor vascular vitamin $\mathrm{K}$ status. Plasma dp-ucMGP was the only marker that correlated with PIVKA-II levels, stressing its value as a vascular biomarker for vitamin $\mathrm{K}$ status. With respect to osteocalcin, both the uncarboxylated OC (ucOC) and carboxylated OC (cOC) levels were high, resulting in a normal osteocalcin ratio. This may be explained by retention of circulating $O C$ (fragments) in uremic serum [59, 64]. An alternative explanation may be that ESRDassociated increased bone turnover resulted in an overall increased $\mathrm{OC}$ synthesis. Further investigations are needed to elucidate why both ucOC and $\mathrm{COC}$ levels measured with ELISA are high in HD patients. Overall, the application of $O C$ measurements in HD patients seems to be complicated and $\mathrm{OC}$ carboxylation may not be a useful marker for vitamin $\mathrm{K}$ status in HD patients [59]. 
In general, dietary vitamin $\mathrm{K}$ intake in healthy subjects and in HD patients is sufficient to maintain normal haemostasis, but may not be sufficient for full carboxylation (activation) of the extra-hepatic Gla-proteins. The present study confirms the findings of Booth and coworkers $[59,60]$, with exception of poor OC carboxylation. It should be noted, however, that OC carboxylation was measured with a different technique in these studies than used in the present study. Their outcomes may therefore not be comparable with ours. It can be hypothesized that vitamin $\mathrm{K}$ deficiency in HD patients is due to a diminished dietary intake, which may increase the risk of arterial calcification. Our study confirms that the dietary vitamin $\mathrm{K}$ intake in HD patients is low, although we could not demonstrate a correlation between vitamin $\mathrm{K}$ intake and markers for vitamin $\mathrm{K}$ status. This might be due to the relatively low number of patients.

The recommended daily allowance (RDA) for vitamin $\mathrm{K}$ of $1 \mu \mathrm{g} / \mathrm{day} / \mathrm{kg}$ bodyweight is based on intakes of vitamin $\mathrm{K}_{1}$ needed to maintain adequate synthesis of blood coagulation factors. The (higher) requirements of extra-hepatic tissues for maximal carboxylation of Gla-proteins were not taken into account in this recommendation, and although vitamin $\mathrm{K}$ intake met this intake level for the majority of HD patients, it is clear that it is too low for maximal carboxylation of extra-hepatic Gla-proteins. Based on the elevated plasma levels of PIVKA-II it may even be concluded that the hepatic vitamin $\mathrm{K}$ status was not adequate to fully support the carboxylation of the vitamin K-dependent clotting factors. The number of patients included in our study is too low to investigate whether factors other than low dietary intake may cause insufficient vitamin $\mathrm{K}$ status and to evaluate its long-term consequences. Further studies need to be performed to confirm the present results in larger cohorts of CKD and dialysis patients, including peritoneal dialysis patients. Also, it may be relevant to evaluate kidney transplant recipients, because these patients may well maintain their previous dietary habits as dialysis patient.

What may be the clinical implications of our findings? Improvement of vitamin K status in $\mathrm{HD}$ patients may readily be achieved by food supplements. The bioavailability of vitamin $\mathrm{K}$ from supplements is probably better than that from most foods [7]. For OC it is known that, although ucOC is responsive to increased dietary vitamin $\mathrm{K}$, a maximal response can only been achieved with pharmacological vitamin $\mathrm{K}$ intakes [7]. The most common vitamin $\mathrm{K}$ form in food supplements and multivitamins is $K_{1}$. Two commercially available $K_{2}$ vitamins are MK-4 and MK-7. The half-life of MK-7 (approximately 3 days), is much longer compared to that of MK-4 and $\mathrm{K}_{1}$, with a steady state plasma level reached after 2 weeks of daily supplementation [65]. To improve the vitamin $K$ status of HD patients, supplementation may be indeed be the best method, since increasing the dietary intake of vitamin $\mathrm{K}$ is probably not possible due to the generally prescribed dietary regimen, as discussed previously. 


\section{General Discussion}

Since HD patients are generally prescribed multivitamins, it may be relatively easy to add vitamin $\mathrm{K}$ to these preparations. A contra-indication for vitamin $\mathrm{K}$ supplementation is the use of vitamin K-antagonists (oral anticoagulants).

In conclusion, this is the first study demonstrating that HD patients have a vitamin $\mathrm{K}$ intake too low to prevent poor overall vitamin $\mathrm{K}$ status. The high dp-ucMGP levels are strongly suggestive for vascular vitamin $\mathrm{K}$ insuffiency to a level that the calcification-inhibitory activity of MGP is impaired, which may contribute to the extremely high risk for arterial calcification in HD patients. These data therefore warrant vitamin $\mathrm{K}$ intervention studies to elucidate the potential clinical benefit of vitamin $\mathrm{K}$ supplementation in HD patients.

\subsection{Vitamin $\mathrm{K}$ antagonists}

Vitamin $\mathrm{K}$ antagonists, also known as oral anticoagulants (OAC), are widely prescribed for the treatment and prevention of thromboembolic diseases. Coumarin derivatives remain the only licensed oral anticoagulant agents for the treatment of venous thromboembolism to this day. Warfarin, a coumarin derivative, was approved in 1954 for medical use. Limitations of OAC include their slow onset of action and the variability in effectiveness between subjects which results in part from genetic polymorphisms in the metabolic pathways of OAC [66]. Moreover, frequent monitoring and dose adjustments are required to maintain the international normalized ratio (INR) within the therapeutic range. OAC block vitamin $\mathrm{K}$ recycling, resulting in undercarboxylated Gla-proteins. Besides coagulation factors, OAC treatment can also affect the extra-hepatic Gla-proteins, including MGP (in cartilage and vasculature) and OC(in bone).

It has been shown in animal models that vitamin $\mathrm{K}$ antagonists induce vascular calcification and cartilage calcification $[21,67,68]$. Several independent human studies investigated the potential association between OAC use and arterial/valvular calcification in humans. Schurgers et al. first addressed this question in patients undergoing aortic valve replacement. Patients treated pre-operative with marcoumar (target INR 2-3, mean duration 25 months) had significantly more valve calcification compared to non-treated patients (mean calcified area $16 \%$ and $37 \%$, respectively; $\mathrm{p}<0.02$ ) [69]. Koos et al. showed that patients undergoing long-term OAC treatment (mean duration 88 months) also had increased CAC (mean score 2,410), compared to non-treated patients (mean score 1,070; $p=0.024)$ [70]. The association between OAC use and soft tissue calcification was confirmed in two other studies. Lerner et al. showed a significant association between the use of warfarin and the risk of calcification in 1155 patients with atrial fibrillation (OR unadjusted $1.71,95 \% \mathrm{Cl} 1.34-2.18$ ), of whom $63 \%$ was treated with warfarin and $37 \%$ without warfarin. 
The association remained significant after adjustment for various confounding risk factors. Further, Rennenberg et al. found a high odds ratio of 8.5 (95\% Cl 2.01-35.95) for calcification in 19 patients receiving coumarins for more than 10 years as compared to 18 matched controls.

The association between OAC treatment and soft tissue calcification has also been investigated in HD patients. Holden et al. assessed the extent of aortic valve calcification in 108 HD patients with echocardiography [71]. The 18 patients who received warfarin for more than 6 months were more likely to have severe aortic valve calcification $(p=0.04)$. There was also a significant association between lifetime months of warfarin exposure and severity of aortic valve calcification in these patients $(p=0.004)$. Long-term warfarin treatment therefore appeared be associated with aortic valve calcification in HD patients. However, the results of these studies need to be confirmed in prospective randomized, placebo-controlled clinical trials.

The most common indication for OAC treatment in HD patients is to maintain vascular access for dialysis treatment [72]. The role of low-intensity OAC treatment (target INR 1.4-1.9) for prevention of vascular access-thrombosis has been investigated in a randomized placebo-controlled trial in 107 patients [73]. This study, however, was cessated after the occurrence of serious bleedings in the warfarin group. The use of warfarin for vascular access therefore seems to confer little benefit to these patients and appears to expose them to an unacceptably high risk of major bleedings [71]. Moreover, the safety and efficacy of OAC treatment in dialysis patients still has to be demonstrated in randomized clinical trials.

Another indication for OAC treatment in dialysis patients is atrial fibrillation. A recent paper by Chan et al. showed in a large retrospective cohort of 1671 incident HD patients (average follow-up 1.6 years) that warfarin use is associated with a significantly increased risk for new (ischemic and hemorrhagic) stroke (HR1.93 compared to non-use; $95 \% \mathrm{Cl}$ 1.29-2.90). Warfarin use was not associated with increased all-cause mortality or hospitalization. The authors discussed that in addition to a hemorrhagic mechanism for stroke, warfarin may potentiate vascular calcification to increase the risk for ischemic stroke. Wizemann et al. also found that warfarin use was associated with a significantly higher stroke risk in HD patients, particularly in those over 75 years of age ( $n=171 ; H R 2.17,95 \%$ $\mathrm{Cl}$ 1.04-4.53, p=0.04). In addition, OAC therapy (warfarin) was not associated with fewer cerebrovascular events. It can be concluded that the effectiveness and safety of warfarin use in HD patients should be further investigated. 


\section{General Discussion}

Cozzolino and Brandenburg have recently reviewed the potential adverse effect of OAC therapy in CKD patients [74]. Patients with calciphylaxis are described as striking evidence for the deleterious role of OAC in inducing ectopic calcification. These patients develop calcification of the skin arteries, resulting in necrotic ulcers, and eventually often death. Brandenburg has registered the use of warfarin in these patients as part of the German section of the International Collaborative Calciphylaxis Network (ICNN) registry. He found that about $50 \%$ of all calciphylaxis patients reported to the registry received treatment with OAC before the onset of symptoms (V. Brandenburg, unpublished data). In cooperation with Brandenburg, we found that calciphylaxis patients had the lowest t-ucMGP levels (Chapter 2 ) and the highest dp-ucMGP values (unpublished data). These results indicate that MGP may be involved in the development of arterial calcification in these patients. Moreover, the high dp-ucMGP levels indicate a poor vascular vitamin $\mathrm{K}$ status, which would be worsened by OAC therapy. It could be hypothesized that these patients would benefit from vitamin $\mathrm{K}$ supplementation. Circulating MGP measurements may contribute to elucidation of the underlying mechanism and to investigation of other treatment strategies.

\subsection{Vitamin $\mathrm{K}$ supplementation}

Whereas vitamin $\mathrm{K}$ antagonists may increase the risk of soft tissue calcification, the use of vitamin $\mathrm{K}$ supplementation may reduce the risk of vascular calcification. The vitamin $\mathrm{K}$ forms that are available in multivitamins or food supplements are vitamin $K_{1}$ (synthetic and the predominant form used), MK-4 (prepared by organic synthesis and almost exclusively used in Japan), and MK-7 (a natural form prepared by extraction of natto food) [65]. After intestinal absorption, $\mathrm{K}$ vitamins are mainly transported in the circulation by lipoproteins to their target tissues. In animal experiments, vitamin $K_{1}$ has been found to preferentially accumulate in the liver, whereas menaquinones had a more widespread distribution pattern [75, 76]. Schurgers et al. demonstrated that during the first hours after oral intake, vitamin $\mathrm{K}_{1}$ and $\mathrm{K}_{2}$ (MK-4 and MK-9, all vitamins dissolved in corn oil) predominantly associated with the triacylglycerol-rich lipoprotein fraction, suggesting that initially most $\mathrm{K}$ vitamins are first transported to the liver [77]. Both menaquinones were also found in the low density-lipoprotein fraction, which is equally transported to extra-hepatic tissues. In the warfarin rat-model, it was shown that MK-4 and not vitamin $\mathrm{K}_{1}$ could inhibit warfarininduced arterial calcification [78]. There was also a higher accumulation of MK-4 in the aortic tissue and a more efficient utilization of MK-4 by the aorta. These results suggest tissue-specific uptake of $\mathrm{K}$ vitamins, in which menaquinones could be more distributed to the extra-hepatic tissues. 
Moreover, Buitenhuis et al. showed that vitamin $\mathrm{K}_{2}$, especially the long chain $\mathrm{K}_{2}$ vitamins such as MK-7, have lower KM values for the enzyme $\mathrm{Y}$-glutamyl carboxylase. Menaquinones may therefore be the preferred cofactors for vascular carboxylase [79]. Recently, Wallin et al. showed that specifically vitamin $\mathrm{K}_{2}$ acts as an anti-calcification component in the vessel wall by increasing the gene expression with a 4.8-fold higher specific activity of DT-diaphorase, an enzyme of the vitamin K-cycle [3]. Menaquinones may therefore have the potential to more effectively influence MGP and arterial calcification than vitamin $\mathrm{K}_{1}$.

Thus far, only two clinical trials have been conducted investigating vitamin $\mathrm{K}$ supplementation and vascular health. The first trial examined the effects of vitamin $K_{1}$ and $D$ supplementation on vascular properties among 181 post-menopausal women aged 55-65 years [80]. Women received placebo, a supplement containing minerals and vitamin D (MD-group), or the same supplement with $1 \mathrm{mg}$ vitamin $\mathrm{K}_{1}$ (MDK-group). The elastic properties of the common carotid artery, assessed with echography, remained unchanged during the three-year study-period, but decreased in the MD- and placebo-group. However, the change in intima-media thickness did not differ between the groups. Shea et al. investigated the effect of $500 \mu \mathrm{g}$ vitamin $\mathrm{K}_{1}$ in a multivitamin (as compared to a multivitamin alone) on the progression of CAC during 3 years in 388 healthy men and postmenopausal women [81]. In a subgroup analysis of participants with an adherence of $\geq 85 \%$ to supplementation ( $n=367$ ), CAC progression was $6 \%$ less in those who received vitamin $K_{1}$. MGP was also measured with a MGP radio-immunoassay, which cannot determine the carboxylation status of MGP. Neither baseline or changes in MGP concentrations predicted the change in CAC. The first placebo-controlled clinical trial investigating the effect of MK-7 supplementation on bone health as well as vascular characteristics is currently being conducted.

Adequate intakes of vitamin K, particularly of menaquinones, could be of importance for the prevention of cardiovascular disease. However, there are only very low amounts of vitamin $\mathrm{K}$ in the diet, with only a few food products containing sufficient amounts of particularly menaquinones. Supplementation could therefore be of major importance. In a comparison of synthetic $\mathrm{K} 1$ and natto-derived MK-7, it was found that the half-life time of MK-7 is very long (several days) compared to that of K1 (several hours), resulting in more stable circulating levels and accumulation of MK-7 to higher levels during prolonged intake [65]. MK-7 is therefore longer available for uptake by extra-hepatic tissues. In addition, MK-7 was more effective in both catalyzing osteocalcin carboxylation and counteracting coumarin-derivatives in the liver. 


\section{General Discussion}

The finding of circulating dp-ucMGP confirms the previous reported finding of uncarboxylated, inactive MGP species in the circulation of healthy subjects [82]. This may indicate that the majority of the healthy population is not optimally protected against calcification, since part of their MGP occurs in an uncarboxylated, inactive form. This has also been reported for $\mathrm{OC}$, of which uncarboxylated levels are also present in the circulation of healthy subjects. The dp-ucMGP levels decreased to almost immeasurably low levels after 6 months of vitamin $\mathrm{K}$ supplementation, indicating that the even in healthy subjects the vascular vitamin $\mathrm{K}$ status is insufficient for maximal MGP carboxylation. 


\section{Value of circulating MGP measurements}

\subsection{For research}

With the t-ucMGP assay, the total pool of uncarboxylated MGP can be measured both in plasma and serum, regardless of the phosphorylation status (Chapter 2). We found consistently low t-ucMGP levels in dialysis patients (Chapter 2 and 4), and showed that t-ucMGP is inversely correlated with arterial calcification in this patient population (Chapter 3 ). The search for biomarkers reflecting or predicting (the risk of) arterial calcification has led to the development of ELISA measuring proteins involved in the calcification process. Circulating t-ucMGP as a biomarker could easily be included in such studies, since it is an important local inhibitor of arterial calcification. Moreover, MGP is the only calcification inhibitor of which the function of MGP can be modulated by either the use of vitamin $\mathrm{K}$ supplementation or vitamin $\mathrm{K}$ antagonists.

For studies in which vascular vitamin $\mathrm{K}$ status needs to measured, dp-ucMGP seems to be particularly suited as a biomarker (Chapter 5 ). The major drawback of this assay is that dpucMGP levels are only measurable in plasma samples. We demonstrated in healthy subjects that dp-ucMGP levels decrease upon vitamin $\mathrm{K}$ supplementation and increase upon vitamin $\mathrm{K}$ antagonist use. In collaboration with Dr. Westenfeld from the department of Cardiology, RWTH Aachen University, a pilot study was performed among 50 HD patients, who received MK-7 supplementation in a dosage of 45,135 , or $360 \mu \mathrm{g} /$ day (R. Westenfeld, submitted for publication). The dp-ucMGP levels significantly decreased after only 6 weeks of supplementation. In the highest dosage-group, the response-rate for dp-ucMGP levels was even $100 \%$. Circulating dp-ucMGP may therefore serve as a biomarker to follow-up vitamin $\mathrm{K}$ status not only in healthy subjects, but also in patient populations. Additionally, dp-ucMGP could serve as a marker for investigating the effect of high vitamin $\mathrm{K}$ intake on the development or progression of arterial calcification. Any changes in $\mathrm{dp}$ ucMGP levels could then be correlated with (changes in) clinical calcification scores. Investigating the effect of vitamin $\mathrm{K}$ intake on vascular calcification has been performed previously [81], however at time no species-specific MGP assay was available. Using dp-ucMGP as a biomarker, it can be investigated whether any (beneficial) effect of high vitamin $\mathrm{K}$ intake works via MGP. Currently, trials investigating the effect of vitamin K supplementation on dp-ucMGP and vascular calcification are in progress. Measurements of dp-ucMGP levels could also contribute to gaining insight in the underlying mechanism between OAC therapy and arterial calcification. Clinical trials investigating this association, can directly link any changes in dp-ucMGP levels with clinical markers for arterial calcification. 


\section{General Discussion}

Thus far, tools to investigate the vascular vitamin $\mathrm{K}$ status were unavailable. Such investigations would contribute to the elucidation of the role of MGP in the association between OAC therapy and arterial calcification.

The development of the MGP assays described in this thesis has led to many collaborations in which we measured circulating MGP species in different patient cohorts. An overview of these and our studies and their major findings is given in Table 1 . These studies included investigations of MGP as a biomarker in vascular pathologies, but also of MGP's role in non-vascular pathologies. Moreover, MGP levels are currently being measured in a large population-based study investigating vitamin $\mathrm{K}$ status, intake, and cardiovascular morbidity and mortality (collaboration between VitaK in Maastricht and the Julius Center in Utrecht, for which a grant of the Dutch Heart Foundation has been received).

Pseudoxanthoma elasticum (PXE) is an outstanding example of a disease in which measurements of MGP have contributed to the knowledge of the calcification process in this pathology. PXE is an autosomal recessive disorder caused by mutations in the $A B C C 6$ gene (ATP-binding cassette subfamily $C$ member 6 ). A characteristic feature of this disease is mineralization of connective tissues, particularly of elastic fibers, affecting mainly the skin, eyes, and cardiovascular system. PXE-like clinical findings have been seen in patients suffering from mutations in either the GGCX or VKOR1 genes [83], which are normally associated with deficiency of the vitamin K-dependent coagulation factors. Staining of skin samples from patients with mutations in the GGCX gene, revealed predominantly the presence of ucMGP species in areas of mineralization in the dermis [83]. Li et al. hypothesized that reduced carboxylase activity in these patients leads to a reduction of MGP carboxylation, allowing peripheral mineralization to occur [83]. The same group demonstrated low t-ucMGP values and high dp-ucMGP levels in a family with ABCC6 and GGCX mutations [84] (Table 1). The involvement of MGP in classic PXE was also demonstrated by this group and confirmed by the group of Gheduzzi, showing that fibroblasts from PXE patients almost exclusively produce the inactive UCMGP, which is not able to block or inhibit calcification $[85,86]$. The very low cMGP production in pathological fibroblasts compared to controls suggests that these cells have an impaired vitamin $\mathrm{K}$ metabolism, which may play an important role in the ectopic calcification in PXE. 
Table 1: Circulating MGP measurements with the t-ucMGP, dp-ucMGP, and dp-cMGP assays

\begin{tabular}{|c|c|}
\hline t-ucMGP assay & Results \\
\hline Hermans et al., Blood Purif 2007 & $\begin{array}{l}\text { Dialysis patients }(n=120) \text { had significantly lower t-ucMGP levels as com- } \\
\text { pared to healthy controls. t-ucMGP was negatively correlated with the } \\
\text { aortic augmentation index, but not with pulse wave velocity or pulse } \\
\text { pressure. }\end{array}$ \\
\hline Van Summeren et al., Pediatr Nephrol 2008 & $\begin{array}{l}\text { Pediatric renal transplant recipients }(n=29) \text { had no different } t \text {-ucMGP levels } \\
\text { as compared to healthy controls. t-ucMGP was not associated with vascu- } \\
\text { lar parameters. }\end{array}$ \\
\hline Shroff et al., Nephrol Dial Transplant 2008 & $\begin{array}{l}\text { Pediatric dialysis patients }(n=61) \text { had significantly lower MGP levels as } \\
\text { compared to healthy controls. t-ucMGP was not associated with vascular } \\
\text { parameters, including CAC scores. }\end{array}$ \\
\hline Cranenburg et al., J Vasc Res 2008 & $\begin{array}{l}\text { Angioplasty }(n=30) \text {, aortic stenosis }(n=25) \text {, hemodialysis }(n=52) \text {, and } \\
\text { calciphylaxis }(n=10) \text { patients had significantly lower t-ucMGP levels as } \\
\text { compared to healthy controls. }\end{array}$ \\
\hline Cranenburg et al., Thromb Haemost 2009 & $\begin{array}{l}\text { Hemodialysis patients }(n=40) \text { had significantly lower t-ucMGP levels as } \\
\text { compared to healthy controls and patients without CAC. t-ucMGP was } \\
\text { inversely associated with CAC scores in hemodialysis patients. }\end{array}$ \\
\hline Parker et al., Nephrol Dial Transplant 2009 & $\begin{array}{l}\text { Among stable CVD patients }(n=842) \text { decreased t-ucMGP levels were } \\
\text { associated with a reduced glomerular filtration rate. }\end{array}$ \\
\hline Koos et al., Thromb Haemost 2009 & $\begin{array}{l}\text { Aortic valve disease patients ( } n=191 \text { ) had significantly lower t-ucMGP levels } \\
\text { compared to control subjects. t-ucMGP was not associated with the } \\
\text { severity of aortic valve disease or calcification. }\end{array}$ \\
\hline Schlieper et al., Kidney Blood Press Res 2009 & $\begin{array}{l}\text { Among hemodialysis patients }(n=188) \text { t-ucMGP was not associated with } \\
\text { semiquantitive cardiovascular calcification scores. }\end{array}$ \\
\hline Parker et al., Atherosclerosis 2010 & $\begin{array}{l}\text { Among stable CVD patients }(n=839) \text { higher t-ucMGP was associated with } \\
\text { lower odds of mitral annular calcification in patients without diabetes, and } \\
\text { higher odds of calcification in patients with diabetes. }\end{array}$ \\
\hline Parker et al., Ann Intern Med 2010 & $\begin{array}{l}\text { Among stable CAD patients }(n=833) \text { those in the highest } t \text {-ucMGP tertile } \\
\text { had a } \sim 50 \% \text { lower mortality risk compared to those in the lowest tertile. }\end{array}$ \\
\hline Rennenberg et al., Eur J Clin Invest 2010 & $\begin{array}{l}\text { Among hypertensive patients }(n=36) \text { t-ucMGP was not associated within } \\
\text { calcification scores in univariate analysis. }\end{array}$ \\
\hline Noordzij et al.,Nephrol Dial Transplant 2010 & $\begin{array}{l}\text { Among hemodialysis and peritoneal dialysis patients ( } n=160) \text { t-ucMGP was } \\
\text { not associated with (progression of) aortic calcification. }\end{array}$ \\
\hline Cranenburg et al., submitted for publication & $\begin{array}{l}\text { Among hemodialysis and peritoneal dialysis patients }(n=952) \text { there was no } \\
\text { difference between t-ucMGP tertiles in overall, cardiovascular, or non- } \\
\text { cardiovascular mortality. t-ucMGP was inversely associated with pulse } \\
\text { pressure. }\end{array}$ \\
\hline
\end{tabular}




\begin{tabular}{|c|c|}
\hline dp-ucMGP and/or dp-cMGP assay & Results \\
\hline Schurgers et al., Clin J Am Soc Nephrol 2010 & $\begin{array}{l}\text { Among CKD patients ( } n=107) \text { dp-uCMGP levels increased with CKD stage } \\
\text { and were positively associated with aortic calcification score. }\end{array}$ \\
\hline Cranenburg et al.,Thromb Haemost 2010 & $\begin{array}{l}\text { Aortic valve disease }(n=50) \text { and hemodialysis }(n=45) \text { patients had signifi- } \\
\text { cantly higher dp-ucMGP and dp-cMGP levels as compared to healthy } \\
\text { controls. dp-ucMGP decreased upon vitamin K supplementation and } \\
\text { increased upon vitamin K antagonist use in healthy subjects ( } n=52 \text { and } \\
n=17) \text {. dp-cMGP decreased upon high-dosage vitamin } K \text { supplementation } \\
(n=28) \text {. }\end{array}$ \\
\hline Schlieper et al., submitted for publication & $\begin{array}{l}\text { Hemodialysis patients ( } n=188 \text { ) had significantly higher dp-ucMGP and dp- } \\
\text { cMGP levels compared to controls. dp-ucMGP was positively associated } \\
\text { with PIVKA-II. Patients with low dp-cMGP levels had higher vascular scores } \\
\text { and an increased all-cause and cardiovascular mortality risk. }\end{array}$ \\
\hline Cranenburg et al., submitted for publication & $\begin{array}{l}\text { Hemodialysis patients ( } n=40 \text { ) had high dp-ucMGP levels, which were } \\
\text { positively associated with PIVKA-II levels. }\end{array}$ \\
\hline Ueland et al., submitted for publication & $\begin{array}{l}\text { Patients with calcific valvular aortic stenosis ( } n=147) \text { had significantly } \\
\text { higher dp-ucMGP and dp-cMGP levels compared to controls. dp-ucMGP } \\
\text { was associated with cardiac function and long-term mortality in multivari- } \\
\text { ate analysis. }\end{array}$ \\
\hline Westenfeld et al., submitted for publication & $\begin{array}{l}\text { Hemodialysis patients }(n=50) \text { had } 4.5 \text { fold higher dp-ucMGP levels com- } \\
\text { pared to controls. dp-ucMGP levels decreased upon } 6 \text { weeks of MK- } 7 \\
\text { supplementation }(45,135 \text {, or } 360 \mu \mathrm{g} / \text { day), with a response rate of } 80 \% \text { in } \\
45 \mu \mathrm{g} \text { group and of } 100 \% \text { in the } 360 \mu \text { group. }\end{array}$ \\
\hline
\end{tabular}

\begin{tabular}{ll}
\hline t-ucMGP, dp-ucMGP and dp-cMGP assays & Results \\
\hline Li et al., J Invest Dermatol 2009 & A family with PXE-like syndrome had low-normal to low t-ucMGP levels as \\
& compared to unrelated controls. Two family members with mutations in \\
& the $G G C X$ gene had high dp-ucMGP levels and a high dp-ucMGP/dp-cMGP \\
& ratio $(>1.0)$, whereas the other family-members had low dp-ucMGP levels \\
& and a ratio of $<1.0$. \\
& PXE patients ( $n=16)$ had significantly lower t-ucMGP levels as compared to \\
& controls. PXE-like patients ( $\mathrm{n}=4$ ) had lower t-ucMGP and higher dp-ucMGP \\
Vanakker et al., Lab Invest 2010 & levels as compared to controls. dp-cMGP levels were not different in \\
& patients. \\
\hline
\end{tabular}

Abbreviations used: CAC, coronary artery calcification; CVD, cardiovascular disease; CKD, chronic kidney disease; PIVKA-II, protein induced by vitamin K absence II; MK-7, menaquinones-7; PXE, pseudoxanthoma elasticum; GGCX, y-glutamyl carboxylase. 


\subsection{For clinical application}

Circulating t-ucMGP was inversely associated with arterial calcification in the dialysis patients (Chapter 3 ) and could therefore contribute to the identification of patients with high probability of cardiovascular calcification and who would benefit from a more thorough diagnostic work-up (e.g. non-invasive ischemia testing or coronary angiography). However, this has not yet been confirmed in patient populations besides. More research needs to be performed before any clinical application can be considered.

Measurements of dp-ucMGP could contribute to the identification of patients with vascular vitamin K-deficiency, possibly at high risk for developing arterial calcification (Chapter 5). First, however, it should be investigated whether subjects with high dp-ucMGP levels are indeed at risk to develop arterial calcification, independent of the presence of traditional atherogenic risk factors or known promoters of arterial calcification (for instance high calcium and phosphate levels). Studies investigating the effect of both vitamin K supplementation and vitamin $\mathrm{K}$ antagonists on the development of arterial calcification, could help in establishing the clinical value of dp-ucMGP measurements. In these intervention studies, it should be investigated whether any decrease or increase in dp-ucMGP is associated with changes in clinical markers for arterial calcification. Until such studies have been conducted, dp-ucMGP measurements cannot be applied in clinical practice. A possible clinical application for dp-ucMGP measurements is in CKD patients to identify those patients who are at high risk for warfarin-induced vascular calcification [74].

Lateral flow tests are now being developed for ucMGP species. Such tests may be used for point of care or even home diagnostics providing information to the consumer about his/her vitamin $\mathrm{K}$ status and the potential benefit of taking vitamin $\mathrm{K}$ supplements. By monitoring MGP carboxylation on a regularly basis, this system would provide a unique possibility to visualize short-term benefits of vitamin supplements. Without doubt, such system should not be brought to the market before the health benefits of nutritional doses of supplemental vitamin $\mathrm{K}$ have been confirmed in long-term clinical trials.

Besides clinical research to further explore the diagnostic value of MGP measurements, more fundamental research is needed to unravel the processes of underlying MGP synthesis, transport, carboxylation, and phosphorylation. Such basic knowledge is needed to explain the value of circulating MGP measurements as marker for local vascular processes. Animal experiments using the MGP null mouse and knocked-in constructs of MGP in which serine residues have been replaced could for instance be conducted to further investigate the role of MGP phosphorylation. On short term, paired plasma and tissue samples would contribute to full understanding of results of the MGP assays. 


\section{General Discussion}

Regarding measurements of circulating MGP species, the mono-antibody t-pMGP assay is currently further investigated in different patient populations. The development of an assay to measure the total circulating MGP pool, using for instance antibodies directed against the $\mathrm{C}$ - terminal end of MGP, would contribute significantly to our understanding of the circulating MGP species. 


\section{Conclusions}

Vascular calcification is a major determinant of cardiovascular mortality and recent data demonstrate that one of the main calcification inhibitors in the vasculature is MGP. The measurement of biomarkers, which can reflect the early signs of vascular disease, could be of great importance. We have developed three different MGP assays to measure various circulating MGP species. The first studies investigating MGP levels in different patient populations are described in this thesis. The t-ucMGP assay has the potential to become a biomarker for prevalent arterial calcification, although this has to be further confirmed in ESRD and other patient populations. The dp-ucMGP assay seems to be particularly suited to assess vascular vitamin $\mathrm{K}$ status and may therefore contribute to cardiovascular risk assessment. In conclusion, based on our results, each MGP assay may have its own merit for research and/or for diagnostic use. Further research is necessary to investigate the full diagnostic potential of the different MGP assays. 


\section{References}

1. Murshed, M., et al., Extracellular matrix mineralization is regulated locally; different roles of two glacontaining proteins. J Cell Biol, 2004. 165(5): p. 625-30.

2. Berkner, K.L., The vitamin K-dependent carboxylase. J Nutr, 2000. 130(8): p. 1877-80.

3. Wallin, R., L.J. Schurgers, and N. Wajih, Effects of the blood coagulation vitamin $K$ as an inhibitor of arterial calcification Thromb Res 2008. 122(3): p. 411-7.

4. Zebboudj, A.F., M. Imura, and K. Bostrom, Matrix GLA protein, a regulatory protein for bone morphogenetic protein-2. J Biol Chem, 2002. 277(6): p. 4388-94.

5. Cranenburg, E.C.M., et al., Uncarboxylated matrix Gla protein (UCMGP) is associated with coronary artery calcification in haemodialysis patients. Thromb Haemost, 2009. 101(2): p. 359-66.

6. Schurgers, L.J., et al., Post-translational modifications regulate matrix Gla protein function: importance for inhibition of vascular smooth muscle cell calcification. J Thromb Haemost, 2007. 5(12): p. 2503-11.

7. Vermeer, C., et al., Beyond deficiency: potential benefits of increased intakes of vitamin $K$ for bone and vascular health. Eur J Nutr, 2004. 43(6): p. 325-35.

8. Blacher, J., et al., Arterial calcifications, arterial stiffness, and cardiovascular risk in end-stage renal disease. Hypertension, 2001. 38(4): p. 938-42.

9. Rodriguez Garcia, M., M. Naves Diaz, and J.B. Cannata Andia, Bone metabolism, vascular calcifications and mortality: associations beyond mere coincidence. J Nephrol, 2005. 18(4): p. 458-63.

10. Lehto, S., et al., Medial artery calcification. A neglected harbinger of cardiovascular complications in noninsulin-dependent diabetes mellitus. Arterioscler Thromb Vasc Biol, 1996. 16(8): p. 978-83.

11. Rennenberg, R.J.M.W., et al., Vascular calcifications as a marker of increased cardiovascular risk: a metaanalysis.Vasc Health Risk Manag, 2009. 5(1): p. 185-97.

12. London, G.M., et al., Arterial media calcification in end-stage renal disease: impact on all-cause and cardiovascular mortality. Nephrol Dial Transplant, 2003. 18(9): p. 1731-40.

13. Asanuma, Y., et al., Premature coronary-artery atherosclerosis in systemic lupus erythematosus. N Engl J Med, 2003. 349(25): p. 2407-15.

14. Wang, S., et al., Prevalence and extent of calcification over aorta, coronary and carotid arteries in patients with rheumatoid arthritis. J Intern Med, 2009. 266(5): p. 445-52.

15. Shanahan, C.M., Vascular calcification--a matter of damage limitation? Nephrol Dial Transplant, 2006. 21(5): p. 1166-9.

16. Johnson, R.C., J.A. Leopold, and J. Loscalzo, Vascular calcification: pathobiological mechanisms and clinical implications. Circ Res, 2006. 99(10): p. 1044-59.

17. Otawara, Y.Y. and P.P.A. Price, Developmental appearance of matrix GLA protein during calcification in the rat. J Biol Chem, 1986. 261(23): p. 10828-32.

18. Braam, L.A., et al., Assay for human matrix gla protein in serum: potential applications in the cardiovascular field. Arterioscler Thromb Vasc Biol, 2000. 20(5): p. 1257-61.

19. Schurgers, L.J., et al., Characteristics and performance of an immunosorbent assay for human matrix Glaprotein. Clin Chim Acta, 2005. 351(1-2): p. 131-8.

20. Schurgers, L.J., et al., Novel conformation-specific antibodies against matrix gamma-carboxyglutamic acid (Gla) protein: undercarboxylated matrix Gla protein as marker for vascular calcification. Arterioscler Thromb Vasc Biol, 2005. 25(8): p. 1629-33.

21. Price, P.A., S.A. Faus, and M.K. Williamson, Warfarin causes rapid calcification of the elastic lamellae in rat arteries and heart valves. Arterioscler Thromb Vasc Biol, 1998. 18(9): p. 1400-7.

22. Sweatt, A., et al., Matrix Gla protein (MGP) and bone morphogenetic protein-2 in aortic calcified lesions of aging rats. J Thromb Haemost, 2003. 1(1): p. 178-85.

23. Robinson, J., et al., Electron-Beam Computerized Tomography Correlates with Coronary Angiogram in Chronic Kidney Disease Patients. Am J Nephrol, 2007. 27(3): p. 247-52.

24. Block, G.A., et al., Mortality effect of coronary calcification and phosphate binder choice in incident hemodialysis patients. Kidney Int, 2007. 71(5): p. 438-41.

25. Koos, R., et al., Relation of circulating Matrix Gla-Protein and anticoagulation status in patients with aortic valve calcification. Thromb Haemost, 2009. 101(4): p. 706-13. 
26. Parker, B.D., et al., Association of kidney function and uncarboxylated matrix Gla protein: Data from the Heart and Soul Study. Nephrol Dial Transplant, 2009. 24(7): p. 2095-2101.

27. Noordzij, M., et al., Progression of aortic calcification is associated with disorders of mineral metabolism and mortality in chronic dialysis patients. Nephrol Dial Transplant, 2010.

28. Rosenhek, R., et al., Predictors of outcome in severe, asymptomatic aortic stenosis. N Engl J Med, 2000. 343(9): p. 611-7.

29. Schurgers, L.J., et al., The circulating inactive form of matrix gla protein is a surrogate marker for vascular calcification in chronic kidney disease: a preliminary report. Clin J Am Soc Nephrol, 2010. 5(4): p. 568-75.

30. Proudfoot, D., et al., Apoptosis regulates human vascular calcification in vitro: evidence for initiation of vascular calcification by apoptotic bodies. Circ Res, 2000. 87(11): p. 1055-62.

31. Hur, D.J., et al., A novel MGP mutation in a consanguineous family: review of the clinical and molecular characteristics of Keutel syndrome. Am J Med Genet A, 2005. 135(1): p. 36-40.

32. Munroe, P.B., et al., Mutations in the gene encoding the human matrix Gla protein cause Keutel syndrome. Nat Genet, 1999. 21(1): p. 142-4.

33. Keutel, J., G. Jorgensen, and P. Gabriel, A new autosomal-recessive hereditary syndrome. Multiple peripheral pulmonary stenosis, brachytelephalangia, inner-ear deafness, ossification or calcification of cartilages. Dtsch Med Wochenschr, 1971. 96(43): p. 1676-81 passim.

34. Say, B., et al., Unusual calcium deposition in cartilage associated with short stature and peculiar facial features: a case report. Pediatr Radiol, 1973. 1(2): p. 127-9.

35. Walbaum, R., et al., Proceedings: Keutel's syndrome. Pediatrie, 1975. 30(3): p. 313-4.

36. Fryns, J.P., et al., Calcification of cartilages, brachytelephalangy and peripheral pulmonary stenosis. Confirmation of the Keutel syndrome. Eur J Pediatr, 1984. 142(3): p. 201-3.

37. Cormode, E.J., M. Dawson, and R.B. Lowry, Keutel syndrome: clinical report and literature review. Am J Med Genet, 1986. 24(2): p. 289-94.

38. Khosroshahi, H.E., et al., Keutel syndrome: a report of four cases. Eur J Pediatr, 1989. 149(3): p. $188-91$.

39. Ziereisen, F., C. De Munter, and N. Perlmutter, The Keutel syndrome. Report of a case and review of the literature. Pediatr Radiol, 1993. 23(4): p. 314-5.

40. Haddad, M.C., et al., Premature tracheobronchial, laryngeal and costochondral cartilage calcification in children. Clin Radiol, 1993. 47(1): p. 52-5.

41. Buchsteiner, I., et al., [Congenital subglottic laryngeal stenosis in 2 brothers with chondrodysplasia syndrome (Keutel-Gabriel syndrome)]. Laryngo- rhino- otologie, 1998. 77(7): p. 363-6.

42. Teebi, A.S., et al., Keutel syndrome: further characterization and review. Am J Med Genet, 1998. 78(2): p. 182-7.

43. Tuysuz, B., et al., Picture of the month. Keutel syndrome. Arch Pediatr Adolesc Med, 1999. 153(7): p. 765-6.

44. Gilbert, B. and D. Lacombe, Keutel syndrome and miscarriages. Am J Med Genet, 1999. 83(3): p. $209-11$.

45. Miller, S.F., Brachytelephalangy with sparing of the fifth distal phalanx: a feature highly suggestive of Keutel syndrome. Pediatr Radiol, 2003. 33(3): p. 186-9.

46. Parmar, H., et al., Petrified ears in a patient with Keutel syndrome: temporal bone CT findings. Pediatr Radiol, 2006. 36(3): p. 241-3.

47. Ozdemir, N., et al., Tracheobronchial calcification associated with Keutel syndrome. Turk J Pediatr, 2006. 48(4): p. 357-61.

48. Meier, M., et al., Tracheobronchial stenosis in Keutel syndrome. Eur Respir J, 2001. 17(3): p. 566-9.

49. Shroff, R.C., et al., Dialysis accelerates medial vascular calcification in part by triggering smooth muscle cell apoptosis. Circulation, 2008. 118(17): p. 1748-57.

50. Kaartinen, M.T., et al., Osteopontin upregulation and polymerization by transglutaminase 2 in calcified arteries of Matrix Gla protein-deficient mice. J Histochem Cytochem, 2007. 55(4): p. 375-86.

51. Geleijnse, J.M., et al., Dietary intake of menaquinone is associated with a reduced risk of coronary heart disease: the Rotterdam Study. J Nutr, 2004. 134(11): p. 3100-5.

52. Gast, G.C., et al., A high menaquinone intake reduces the incidence of coronary heart disease. Nutr Metab Cardiovasc Dis, 2009. 19(7): p. 504-10.

53. Beulens, J.W., et al., High dietary menaquinone intake is associated with reduced coronary calcification. Atherosclerosis, 2009. 203(2): p. 489-93. 
54. Jie, K.S., et al., Vitamin K intake and osteocalcin levels in women with and without aortic atherosclerosis: a population-based study. Atherosclerosis, 1995. 116(1): p. 117-23.

55. Villines, T.C., et al., Vitamin K1 intake and coronary calcification. Coron Artery Dis, 2005. 16(3): p. 199-203.

56. Booth, S.L., Roles for vitamin K beyond coagulation. Annu Rev Nutr, 2009. 29: p. 89-110.

57. Braam, L., et al., Dietary phylloquinone intake as a potential marker for a heart-healthy dietary pattern in the Framingham Offspring cohort. J Am Diet Assoc, 2004. 104(9): p. 1410-4.

58. Stein, G., H. Sperschneider, and S. Koppe, Vitamin levels in chronic renal failure and need for supplementation. Blood Purif, 1985. 3(1-3): p. 52-62.

59. Pilkey, R.M., et al., Subclinical vitamin K deficiency in hemodialysis patients. Am J Kidney Dis, 2007. 49(3): p. 432-9.

60. Holden, R.M., et al., Vitamins $k$ and $d$ status in stages 3-5 chronic kidney disease. Clin J Am Soc Nephrol, 2010. 5(4): p. 590-7.

61. Krueger, T., et al., Vitamin $K$ deficiency in CKD patients: a modifiable risk factor for vascular calcification? Kidney Int, 2009. 76(1): p. 18-22.

62. Maas, A.H., et al., Vitamin K intake and calcifications in breast arteries. Maturitas, 2007. 56(3): p. 273-9.

63. Beto, J.A. and V.K. Bansal, Medical nutrition therapy in chronic kidney failure: integrating clinical practice guidelines. J Am Diet Assoc, 2004. 104(3): p. 404-9.

64. Urena, P. and M.C. De Vernejoul, Circulating biochemical markers of bone remodeling in uremic patients. Kidney Int, 1999. 55(6): p. 2141-56.

65. Schurgers, L.J., et al., Vitamin K-containing dietary supplements: comparison of synthetic vitamin K1 and natto-derived menaquinone-7. Blood, 2007. 109(8): p. 3279-83.

66. Ruff, C.T. and E. Braunwald, Will Warfarin Ever Be Replaced? J Cardiovasc Pharmacol Ther, 2010: p. 210-9..

67. Price, P.A., S.A. Faus, and M.K. Williamson, Warfarin-induced artery calcification is accelerated by growth and vitamin D. Arterioscler Thromb Vasc Biol, 2000. 20(2): p. 317-27.

68. Howe, A.M. and W.S. Webster, The warfarin embryopathy: a rat model showing maxillonasal hypoplasia and other skeletal disturbances. Teratology, 1992. 46(4): p. 379-90.

69. Schurgers, L.J., et al., Oral anticoagulant treatment: friend or foe in cardiovascular disease? Blood, 2004. 104(10): p. 3231-2.

70. Koos, R., et al., Relation of oral anticoagulation to cardiac valvular and coronary calcium assessed by multislice spiral computed tomography. Am J Cardiol, 2005. 96(6): p. 747-9.

71. Holden, R.M., et al., Warfarin and aortic valve calcification in hemodialysis patients. J Nephrol, 2007. 20(4): p. 417-22.

72. Bennett, W.M., Should dialysis patients ever receive warfarin and for what reasons? Clin J Am Soc Nephrol, 2006. 1(6): p. 1357-9.

73. Crowther, M.A., et al., Low-intensity warfarin is ineffective for the prevention of PTFE graft failure in patients on hemodialysis: a randomized controlled trial. J Am Soc Nephrol, 2002. 13(9): p. 2331-7.

74. Cozzolino, M. and V. Brandenburg, Warfarin: to use or not to use in chronic kidney disease patients? J Nephrol, 2010.

75. Thijssen, H.H., M.J. Drittij-Reijnders, and M.A. Fischer, Phylloquinone and menaquinone-4 distribution in rats: synthesis rather than uptake determines menaquinone-4 organ concentrations. J Nutr, 1996. 126(2): p. 537-43.

76. Ronden, J.E., H.H. Thijssen, and C. Vermeer, Tissue distribution of K-vitamers under different nutritional regimens in the rat. Biochim Biophys Acta, 1998. 1379(1): p. 16-22.

77. Schurgers, L.J. and C. Vermeer, Differential lipoprotein transport pathways of K-vitamins in healthy subjects. Biochim Biophys Acta, 2002. 1570(1): p. 27-32.

78. Spronk, H.M., et al., Tissue-specific utilization of menaquinone-4 results in the prevention of arterial calcification in warfarin-treated rats. J Vasc Res, 2003. 40(6): p. 531-7.

79. Buitenhuis, H.C., B.A. Soute, and C. Vermeer, Comparison of the vitamins $K 1, K 2$ and $K 3$ as cofactors for the hepatic vitamin K-dependent carboxylase. Biochim Biophys Acta, 1990. 1034(2): p. 170-5.

80. Braam, L.A., et al., Beneficial effects of vitamins $D$ and $K$ on the elastic properties of the vessel wall in postmenopausal women: a follow-up study. Thromb Haemost, 2004. 91(2): p. 373-80.

81. Shea, M.K., et al., Vitamin $K$ supplementation and progression of coronary artery calcium in older men and women. Am J Clin Nutr, 2009. 89(6): p. 1799-807. 
82. Cranenburg, E.C., et al., The circulating inactive form of Matrix Gla Protein (ucMGP) as a biomarker for cardiovascular calcification J Vasc Res 2008. 45: p. 427-36.

83. Li, Q., et al., Co-existent pseudoxanthoma elasticum and vitamin K-dependent coagulation factor deficiency: compound heterozygosity for mutations in the GGCX gene. Am J Pathol, 2009. 174(2): p. 534-40.

84. Li, Q., et al., Mutations in the GGCX and ABCC6 genes in a family with pseudoxanthoma elasticum-like phenotypes. J Invest Dermatol, 2009. 129(3): p. 553-63.

85. Li, Q., et al., Pseudoxanthoma elasticum: reduced gamma-glutamyl carboxylation of matrix gla protein in a mouse model (Abcc6-/-). Biochem Biophys Res Commun, 2007. 364(2): p. 208-13.

86. Gheduzzi, D., et al., Matrix Gla protein is involved in elastic fiber calcification in the dermis of pseudoxanthoma elasticum patients. Lab Invest, 2007. 87(10): p. 998-1008. 


\section{Samenvatting}

Matrix $\gamma$-carboxyglutamate (Gla)-proteïne (MGP) is een klein eiwit dat het proces van arteriële verkalking (vaatverkalking) remt (Hoofdstuk 1). Vaatverkalking leidt tot cardiovasculaire morbiditeit en mortaliteit in de gezonde populatie alsook in hoog-risico patiëntenpopulaties, zoals patiënten met chronische nierinsufficiëntie.

MGP is aanwezig in de vaatwand en in kraakbeen, waar het gesynthetiseerd wordt door vasculaire gladde spiercellen en chondrocyten, respectievelijk. MGP kan twee posttranslationele modificaties ondergaan: $\gamma$-carboxylering van glutamaat residuen en fosforylering van serine residuen. Door $\mathrm{\gamma}$-carboxylering kunnen vitamine $\mathrm{K}$-afhankelijke eiwitten (zgn. Gla-eiwitten) calcium binden, hetgeen essentieel is voor de functie van deze eiwitten. De precieze rol van de phosphoserine residuen is onbekend, maar deze lijken een rol te spelen in de secretie van MGP en in het binden van onoplosbare calciumzouten. Als een resultaat van deze modificaties, kunnen er verschillende MGP species onderscheiden worden: gefosforyleerd MGP (phosphorylated, pMGP), niet-gefosforyleerd MGP (desphospho, dpMGP), gecarboxyleerd MGP (cMGP) en niet-gecarboxyleerd (uncarboxylated, UCMGP) MGP. Door de ontwikkeling van antilichamen gericht tegen specifieke MGP species, werd het mogelijk om MGP species in weefsel en bloed te meten met behulp van immunohistochemie en enzyme-linked immunoassays (ELISA), respectievelijk.

Vitamine $\mathrm{K}$ is een cofactor voor het enzyme $\mathrm{\gamma}$-glutamyl carboxylase, waardoor de carboxyleringsreactie kan plaatsvinden. Deze reactie is gekoppeld aan de recycling van vitamine $\mathrm{K}$. Vitamine $\mathrm{K}$ is aanwezig in groene bladgroenten (vitamine $\mathrm{K}_{1}$, fyllochinon) en in kaas, kwark en het Japanse natto (vitamine $\mathrm{K}_{2}$, menachinonen). Gla-eiwitten hebben verschillende functies in het lichaam, waaronder als bloedstollingsfactoren in de lever. In de extra-hepatische weefsels zijn ook Glaeiwitten aanwezig, zoals MGP in de vaatwand en osteocalcine in bot. De aanbevolen dagelijkse hoeveelheid van vitamine $\mathrm{K}$ is $1 \mathrm{\mu g} / \mathrm{dag} / \mathrm{kg}$ lichaamsgewicht; deze aanbeveling is gebaseerd op de benodigde hoeveelheid vitamin $\mathrm{K}$ voor een adequate bloedstolling.

Arteriële verkalking wordt tegenwoordig als een actief, cel-gemedieerd proces beschouwd. De vasculaire gladde spiercel, welke kan transformeren in een cel gelijkend op de osteoblast/chondrocyt, speelt een centrale rol in dit proces. De balans tussen promotors en remmers van calcificatie lijkt een belangrijke rol te spelen in de ontwikkeling en het voortschrijden van arteriële verkalking. Circulerend MGP is mogelijk een biomarker voor reeds aanwezige vaatverkalking en kan mogelijk patiënten identificeren met een hoog risico op het ontwikkelen hiervan. Het doel van de studies beschreven in dit proefschrift was om ELISA's te ontwikkelen waarmee circulerende MGP species gemeten kunnen worden en om de toepassingsmogelijkheden hiervan te onderzoeken. 
In onze eerste studie (Hoofdstuk 2) ontwikkelden we een ELISA voor het meten van totaal circulerend ucMGP (t-ucMGP). ucMGP species accumuleren op plaatsen van arteriële verkalking in de vaatwand en hebben waarschijnlijk een hoge affiniteit voor hydroxyapatiet. Circulerend t-ucMGP kan daardoor verlaagd zijn in de aanwezigheid van vaatverkalking. De onderzochte patiëntenpopulaties waren patiënten die recent percutane transluminale coronaire angioplastiek (PTCA) hadden ondergaan, patiënten met aortaklep stenose, hemodialyse (HD) patiënten en patiënten gediagnosticeerd met calciphylaxis. Circulerend t-ucMGP was verlaagd in al deze populaties, waarbij er bij de PTCA en aortaklep stenose patiënten een duidelijke overlap was met de t-ucMGP waarden van de gezonde populatie. Bijna alle HD en calciphylaxis patiënten hadden lagere t-ucMGP waarden in vergelijking met de gezonde populatie. Circulerend t-ucMGP is daardoor een marker die in staat is een onderscheid te maken tussen gezonde personen en patiënten met eindstadium nierfalen. Onze hypothese van verlaagd circulerend t-ucMGP bij de aanwezigheid van vaatverkalking werd door deze resultaten ondersteund. Deze studie gaf een eerste aanwijzing dat circulerend t-ucMGP een niet-invasieve biomarker voor cardiovasculaire verkalking kan zijn.

Om de waarde van t-ucMGP als een biomarker voor cardiovasculaire verkalking vast te stellen, onderzochten we de correlatie tussen circulerend t-ucMGP en de ernst van vasculaire verkalking. Daarvoor maten we de t-ucMGP concentraties in het serum van 40 hemodialyse (HD) patiënten (Hoofdstuk 3). Bij deze patiënten werd de hoeveelheid verkalking van de coronairarteriën (coronary artery calcification, $C A C$ ) vastgesteld door middel van een multislice CT (MSCT) scan. Een hoge CAC score is geassocieerd met de aanwezigheid van significante, stenotische laesies in de coronairarteriën alsmede met toegenomen mortaliteit in HD patiënten.

De hoeveelheid circulerend t-ucMGP was significant lager in HD patiënten in vergelijking met gezonde personen van dezelfde leeftijd en (reumatoïde artritis) patiënten zonder CAC. De HD patiënten werden ingedeeld in tertielen van CAC score (CAC $\leq 103.0,103.1$ 600.0 en $\geq 600.1$ ). De patiënten in het laagste tertiel (dus met de minste cardiovasculaire verkalking) hadden significant hogere t-ucMGP concentraties in vergelijking met patiënten in het intermediaire en hoogste tertielen ( $p=0.022$ en $p=0.021$, respectievelijk). Tevens bestond er een inverse correlatie tussen $t$-ucMGP en CAC scores $(r=-0.414, p=0.009)$. Deze correlatie persisteerde na correctie voor leeftijd, duur van dialyse en ultra-sensitief Creactief proteïne, drie belangrijke factoren die een rol spelen bij de ontwikkeling van CAC in patiënten met eindstadium nierfalen. Deze studie bevestigt dat t-ucMGP concentraties laag zijn in patiëntenpopulaties met een hoog risico op cardiovasculaire verkalking (HD en calciphylaxis patiënten). Bovendien laat deze studie zien dat t-ucMGP mogelijk als maker kan dienen om de individuele hoeveelheid CAC in te schatten. 
In Hoofdstuk 4 beschrijven we t-ucMGP metingen in 949 patiënten van NECOSAD, een groot prospectief Nederlands cohort van nieuwe HD en pertioneaal dialyse (PD) patiënten. Deze patiënten werden gevolgd vanaf de start van dialyse tot aan transplantatie of overlijden (inclusieperiode tussen 1997 en 2005, eind van studieperiode Mei 2009). Circulerend t-ucMGP werd gemeten in serum afgenomen na drie maanden dialyse. Patiënten werden ingedeeld in tertielen van t-ucMGP concentraties (hoog, midden en laag) om te onderzoeken of er een verschil in mortaliteit bestond tussen deze tertielen. Bijna alle patiënten (>96\%) hadden t-ucMGP concentraties onder de normaal range, waarbij HD patiënten de laagste concentraties hadden. De gemeten lage t-ucMGP concentraties zijn in overeenstemming met onze eerdere resultaten. Er werd geen significant verschil in totale mortaliteit, cardiovasculaire en niet-cardiovasculaire mortaliteit gevonden tussen de t-ucMGP tertielen. Deze resultaten duiden erop dat patiënten die recent met dialyse begonnen zijn ( 3 maanden geleden), al lage t-ucMGP concentraties hebben. Circulerend tucMGP is daarom minder geschikt als voorspeller voor mortaliteit in deze patiëntenpopulatie.

De carboxylering van MGP is afhankelijk van de aanwezigheid van vitamine K. Bepaalde MGP species zouden daarom de beschikbaarheid van vitamine $K$ in de vaatwand (vasculaire vitamine $K$ status) kunnen weerspiegelen. Klinisch zou een insufficiënte vasculaire vitamine $\mathrm{K}$ status kunnen leiden tot een verhoogd risico op arteriële verkalking. In Hoofdstuk 5 beschrijven we twee nieuwe ELISA's welke dp-ucMGP en dp-cMGP meten. Deze MGP species bevatten geen fosfoserine residuen en lijken daarom geschikt te zijn om de vasculaire vitamine $K$ status te weerspiegelen. Door de afwezigheid van deze residuen komen dp-ucMGP en dp-cMGP gemakkelijker in de circulatie, onafhankelijk van de aanwezigheid van arteriële verkalking. Voornamelijk dp-ucMGP bleek inderdaad gevoelig te zijn voor veranderingen in de vitamine $\mathrm{K}$ status: gezonde personen die vitamine $\mathrm{K}$ supplementen ontvingen hadden na enkele weken significant afgenomen dp-ucMGP concentraties. Proefpersonen die vitamine $\mathrm{K}$ antagonisten (acenocoumarol) kregen gedurende vier weken, hadden daarentegen significant toegenomen dp-ucMGP concentraties.

We vergeleken ook de commercieel verkrijgbare MGP assay (t-dpMGP assay) en de assays ontwikkeld door onze onderzoeksgroep (t-ucMGP, dp-ucMGP en dp-cMGP assays) met elkaar. Hiervoor werden MGP concentraties in gezonde proefpersonen en in een aantal patiëntenpopulaties gemeten met deze assays. Er werden grote verschillen gevonden tussen de MGP assays, wat verklaard kan worden door de verschillende MGP species die door de assays gemeten worden. Hierdoor kunnen er verscheidende aspecten van het MGP systeem onderzocht worden en mogelijk heeft iedere MGP assay een eigen toepassing voor onderzoek en/of diagnostiek. Van alle MGP assays was de dp-ucMGP assay het meest geschikt om de vasculaire vitamine $\mathrm{K}$ status te onderzoeken. 
Keutel syndroom (KS) is een zeldzame, autosomaal-recessieve aandoening veroorzaakt door mutaties in het MGP gen. Wij onderzochten twee patiënten met $\mathrm{KS}$, waaronder een recent geïdentificeerde Nederlandse KS patiënt bij wie een nieuwe homozygote $M G P$ mutatie (c.61+1G>A) gevonden werd (Hoofdstuk 6). Onderzoek van het fenotype van deze patiënt liet abnormale kraakbeenverkalking zien, het belangrijkste kenmerk van KS. Vasculair onderzoek, waaronder een MSCT scan van de coronairarteriën, liet geen aanwijzingen zien voor cardiovasculaire verkalking. Het fenotype van KS patiënten, voornamelijk gekarakteriseerd door kraakbeenverkalking, is niet overeenkomstig met het vaatverkalkings-fenotype van MGP knockout muizen.

Circulerende MGP species werden gemeten in de Nederlandse KS patiënt met behulp van onze MGP assays, waaronder een zeer recent ontwikkelde assay om totaal gefosforyleerd ( $t$ pMGP) te meten. De patiënt had lage dp-ucMGP concentraties, maar de hoogste t-pMGP concentraties in vergelijking met zijn familieleden en gezonde personen. Vitamine $\mathrm{K}$ supplementatie had geen significante invloed op de gemeten MGP concentraties. Dit kan verklaard worden door de MGP mutatie die resulteert in een splice-site mutatie, waaarbij alleen de eerste 14 aminozuren van het gemuteerde eiwit overeenkomstig het normale MGP eiwit zijn. Hierdoor is $\gamma$-carboxylering waarschijnlijk niet mogelijk. Gefosforyleerd MGP was ook aanwezig in de weefsels van de tweede onderzochte KS patiënt verkregen bij postmortem onderzoek. Deze bevinding wijst op mogelijk resterende MGP activiteit en kan een verklaring zijn voor het verschil tussen het fenotype van KS patiënten en van MGP knockout muizen. Er is echter duidelijk meer onderzoek nodig naar de functie van MGP fosforylering.

In onze laatste studie onderzochten we de vitamine $\mathrm{K}$ inname en status van $40 \mathrm{Neder}$ landse HD patiënten (Hoofdstuk 7). Gebaseerd op eerdere studies, was de verwachting dat de vitamine $\mathrm{K}$ inname van deze patiënten laag zou zijn, wat kan bijdragen aan een slechte vitamine $\mathrm{K}$ status en mogelijk aan de ontwikkeling van arteriële verkalking. $\mathrm{Er}$ werd inderdaad een lage vitamine $\mathrm{K}$ inname gevonden in HD patiënten vergeleken met de eerder beschreven vitamine $\mathrm{K}$ inname van de algemene Nederlandse bevolking (>225 $\mu \mathrm{g} / \mathrm{dag})$. Dit kan gerelateerd zijn aan het dieet dat vaak aan HD patiënten voorgeschreven wordt, wat o.a. een restrictie van natrium en kalium omvat.

De vitamine K status van zowel de lever als van extra-hepatische organen werd onderzocht door middel van verschillende serum en plasma bepalingen, waaronder protein induced by vitamin K absence II (PIVKA-II) en dp-ucMGP. Hoge PIVKA-II concentraties werden gevonden in het merendeel van de patiënten, duidend op een relatief tekort aan vitamine $K$ in de lever om volledig gecarboxyleerde stollingsfactoren te produceren. 
De hoge dp-ucMGP concentraties gevonden in alle HD patiënten bevestigen onze eerdere bevindingen in deze patiëntenpopulatie en wijst op een slechte vasculaire vitamine $K$ status. Dus zowel de vitamine K status van de lever als extra-hepatische organen was niet goed.

De vitamine $\mathrm{K}$ inname van gezonde personen en van HD patiënten lijkt voldoende te zijn voor normale hemostase, maar is waarschijnlijk onvoldoende voor volledige carboxylering (activering) van extra-hepatische Gla-eiwitten. Onze studie bevestigt dat de vitamine $\mathrm{K}$ inname van HD patiënten laag is, hoewel er geen correlatie tussen vitamine $K$ inname en markers voor vitamine $\mathrm{K}$ status aantoonbaar was. De hoge dp-ucMGP concentraties zijn sterk suggestief voor vasculaire vitamine $K$ insufficiëntie waardoor de activiteit van MGP beperkt wordt, wat kan bijdragen aan het hoge risico op arteriële verkalking van HD patienten. $\mathrm{Er}$ is meer onderzoek met supplementatie van vitamine $\mathrm{K}$ nodig om de klinische effecten hiervan te beoordelen.

Samenvattend, is vaatverkalking gecorreleerd aan cardiovasculaire mortaliteit en een van de belangrijkste remmers aanwezig in de vaatwand is MGP. Het bepalen van biomarkers, die de eerste tekenen van vaatziekte kunnen weergeven, is hierbij belangrijk. Wij hebben drie verschillende MGP assays ontwikkeld om verschillende circulerende MGP species te meten (Hoofdstuk 8). De t-ucMGP assay kan een biomarker voor prevalente vaatverkalking worden, hoewel dit bevestigd moet worden in patiënten met eindstadium nierfalen en andere patiëntenpopulaties. De dp-ucMGP assay is met name geschikt om vasculaire vitamine $\mathrm{K}$ status te beoordelen en kan hierdoor bijdragen aan het bepalen van het cardiovasculaire risico. In conclusie, gebaseerd op onze resultaten heeft iedere MGP assay zijn eigen waarde voor onderzoek en/of voor klinische diagnostiek. Meer onderzoek is nodig om deze waarde voor de individuele MGP assays vast te stellen. 


\section{Summary}

Matrix $\gamma$-carboxyglutamate (Gla)-protein (MGP) is a small protein that inhibits arterial calcification (Chapter 1). Arterial calcification is associated with cardiovascular morbidity and mortality in the general population, as well as in high-risk patient populations including patients with chronic kidney disease.

MGP is present in the vascular wall and in cartilage, where it is synthesized by vascular smooth muscle cells (VSMC) and chondrocytes, respectively. MGP can undergo two post-translational modifications: $p$-carboxylation of specific glutamate (Glu) residues and phosphorylation of serine residues. $\gamma$-Carboxylation confers calcium-binding properties to vitamin K-dependent proteins and is thought to be essential for the function of these proteins. The exact role of phosphoserine residues is unknown, but it has been suggested that they play a role in the secretion of MGP into the extracellular matrix. We have hypothesized that these negatively-charged groups are also able to bind to insoluble calcium salts. As a result of both post-translational modifications, several MGP species can be synthesized: phosphorylated (pMGP), non-phosphorylated (desphospho, dpMGP), carboxylated (cMGP), or uncarboxylated (ucMGP). With the development of speciesspecific antibodies, it became possible to detect MGP species in tissues by immunohistochemical staining and in the circulation by enzyme-linked immunoassays (ELISA).

Vitamin $\mathrm{K}$ serves as a cofactor for the enzyme $\gamma$-glutamyl carboxylase, thereby promoting the carboxylation reaction that is coupled to vitamin $\mathrm{K}$ recycling. Vitamin $\mathrm{K}$ is present in green leafy vegetables (vitamin $K_{1}$, phylloquinone) and in cheese, curd, and the Japanese food natto (vitamin $K_{2}$, menaquinones). Vitamin K-dependent proteins (Gla proteins) exert a broad range of functions, for example as blood coagulation factors in the liver. Glaproteins are also present in extra-hepatic tissues, including the vasculature (MGP) and bone (osteocalcin, OC). The recommended daily allowance of vitamin $\mathrm{K}$ is $1 \mu \mathrm{g} / \mathrm{day} / \mathrm{kg}$ bodyweight, which is based on requirements for normal haemostasis.

Arterial calcification is currently regarded as an active, cell-mediated process. The VSMC with its ability to transform into osteoblastic/chondrocytic-like cell plays a central role in this process. The balance between promoters and inhibitors of calcification is thought to play an important role in the development and progression of arterial calcification.

Circulating MGP levels may serve as a biomarker for prevalent arterial calcification and for identification of patients at high risk to develop arterial calcification. The aim of the studies described in this thesis was to develop enzyme-linked immunosorbent assays (ELISA) to measure circulating MGP species and to investigate their potential for research and clinical use. 
In our first study (Chapter 2) we developed a single-antibody ELISA for circulating total ucMGP ( $t$-ucMGP) species using a monoclonal ucMGP antibody. ucMGP species accumulate at sites of arterial calcification in the vascular wall and probably have a high affinity for hydroxyapatite. We therefore hypothesized that circulating t-ucMGP levels may be decreased in the presence of arterial calcification. All patient populations, which included coronary angioplasty, aortic valve stenosis, dialysis, and calciphylaxis patients, had significantly lower t-ucMGP levels compared to healthy subjects of the same age. In the coronary angioplasty and aortic stenosis patients there was some overlap with t-ucMGP levels of healthy subjects, which may be explained by the expected larger inter-individual variation in the extent and type of vascular calcification in these patient populations. In the hemodialysis and calciphylaxis patient populations, almost all patients had lower t-ucMGP levels compared to the healthy subjects. This indicated that that t-ucMGP is a marker capable of discriminating between healthy subjects and patients with end-stage renal disease (ESRD). Our hypothesis of low circulating t-ucMGP levels in the presence of arterial calcification was supported by these results. This study indicates that circulating t-ucMGP has the potential to become a non-invasive biomarker for cardiovascular calcification.

To further establish the potential of t-ucMGP as a biomarker for cardiovascular calcification, we aimed to investigate the correlation between t-ucMGP levels and the extent of vascular calcification. We therefore measured t-ucMGP levels in 40 patients undergoing regular hemodialysis (HD) treatment (Chapter 3 ). The extent of coronary artery calcification (CAC) was quantified in these patients using multislice computed tomography (MSCT) scanning. A high CAC score is associated with the severity of coronary artery disease and the presence of significant, stenotic lesions as well as with increased mortality in HD patients. Circulating t-ucMGP levels were significantly lower in HD patients compared to healthy subjects of the same age and patients who had no CAC. This latter group consisted of patients with rheumatoid arthritis in whom MSCT scanning excluded the presence of CAC. HD patients were divided into tertiles according to CAC score (CAC $\leq 103.0$, 103.1 to 600.0 , and $\geq 600.1$ ). Those in the low CAC tertile had significantly higher t-ucMGP levels compared to patients in the intermediate and high tertile $(p=0.022$ and $p=0.021$, respectively). In addition, t-ucMGP levels correlated inversely with CAC scores ( $r=-0.414$, $p=0.009$ ). This correlation persisted after adjustment for age, dialysis vintage, and highsensitivity C-reactive protein, three important confounders regarding CAC development in ESRD patients. This study confirms that t-ucMGP levels are low in patient populations with a high burden of vascular calcification (HD and calciphylaxis patients). Moreover, t-ucMGP measurements may not only be used for inter-group comparisons of arterial calcification, but also for estimating the individual extent of CAC. 
In Chapter 4 we describe t-ucMGP measurements in 949 patients from NECOSAD, a large prospective Dutch cohort of incident HD and peritoneal dialysis (PD) patients. These patients were followed from initiation of dialysis treatment until transplantation or death (enrolment between 1997 and 2005, end of study period May 2009). Circulating t-ucMGP levels were measured in serum samples obtained at the time of 3 months of dialysis treatment. Patients were divided into tertiles according to t-ucMGP levels (high, medium, and low) to determine whether there was a difference in mortality risk between the tertiles. Almost all patients (>96\%) had circulating t-ucMGP levels below the normal range, with HD patients having the lowest t-ucMGP levels. The circulating low t-ucMGP levels were in accordance with our previous results. No significant differences were found between the high, intermediate, and low t-ucMGP tertiles in overall, cardiovascular, and non-cardiovascular mortality. These results indicate that patients who started recently with dialysis treatment ( 3 months) already have low t-ucMGP levels. Circulating t-ucMGP is less suited for predicting mortality in this patient population, probably due to very low t-ucMGP levels in dialysis patients.

Since MGP carboxylation is vitamin K-dependent, certain species may also reflect the availability of vitamin $\mathrm{K}$ present in the vascular wall (vascular vitamin $\mathrm{K}$ status). Clinically, an insufficient vascular vitamin $\mathrm{K}$ status may result in an increased risk for arterial calcification. In Chapter $\mathbf{5}$ we describe two novel dual-antibody assays detecting circulating dpucMGP and dp-cMGP. These species may be suited to reflect the vascular vitamin $\mathrm{K}$ status, since phosphoserine residues are absent in these MGP species. We hypothesized that they would therefore be more easily set free into the circulation, independent of the presence of calcification and mainly influenced by the availability of vitamin K. Particularly circulating dp-ucMGP was found to be sensitive for changes in vitamin $\mathrm{K}$ status: healthy subjects receiving vitamin $\mathrm{K}$ supplementation during several weeks had significantly decreased dp-ucMGP levels, whereas subjects who received vitamin $\mathrm{K}$ antagonists (acenocoumarol) during 4 weeks had significantly increased dp-ucMGP levels.

We also compared the commercially available MGP assay (t-dpMGP assay) and the assays developed by our group (t-ucMGP, dp-ucMGP, and dp-cMGP assay) by measuring MGP levels in healthy subjects and several patient populations with these assays. Overall, major differences were found between the MGP assays, which may be explained by the different fractions detected by the assays of the total circulating MGP pool. These different MGP assays allow the assessment of various aspects of the MGP system and each assay may have its own application for research and/or for diagnostic use. From these assays, the dp-ucMGP assay was particularly suited to assess the vascular vitamin $\mathrm{K}$ status. 
Keutel syndrome (KS) is a rare autosomal recessive disorder resulting from mutations in the MGP gene. We performed a study in two patients with $\mathrm{KS}$, including a newly identified Dutch patient (Chapter 6). A novel homozygous MGP mutation (c.61+1G>A) was identified in this patient, of whom the phenotype was carefully characterized. CT scanning showed abnormal cartilage calcification, which is the hallmark of KS. Vascular evaluation of the patient, including MSCT scanning of the coronary arteries, revealed no signs of arterial calcification. The phenotype of KS patients, mainly characterized by cartilage calcification, seems to be in contrast with the arterial calcification phenotype of the MGP null mice.

We characterized circulating MGP species in the Dutch KS patient, using our speciesspecific MGP assays, including a very recently developed mono-antibody assay to measure phosphorylated MGP species (t-pMGP). The patient had low dp-ucMGP levels, but the highest t-pMGP levels, compared to his relatives and healthy subjects. Vitamin $\mathrm{K}$ supplementation did not measurably affect MGP levels. This may be explained by the finding that the MGP mutation results in a splice-site mutation, with only the first 14 amino-acids of the mutated protein being comparable with those of the mature MGP protein. $\gamma$ Carboxylation may therefore not be possible. Phosphorylated MGP was also found to be present in tissues of the second KS patient, obtained at post-mortem examination. This finding indicates that some residual MGP activity might be present, which could one explanation for the difference between the phenotype of KS patients and MGP null mice. However, further investigation of MGP phosphorylation is needed.

In our last study we investigated the vitamin $\mathrm{K}$ intake and status in a Dutch cohort of 40 HD patients (Chapter 7). Based on previous studies, it was expected that vitamin $\mathrm{K}$ intake of these patients was low, which could contribute to a poor vitamin K status and possibly to the development of arterial calcification. We found a relatively low vitamin $\mathrm{K}$ intake in the HD patients compared to intakes previously found in the general population of the Netherlands ( $>225 \mu \mathrm{g} /$ day). This may be related to the dietary regimen generally prescribed for HD patients, which includes restriction of sodium and potassium intake.

Hepatic as well as extra-hepatic vitamin $\mathrm{K}$ status was investigated with several serum and plasma measurements, including protein induced by vitamin $\mathrm{K}$ absence II (PIVKA-II) and dpucMGP. High PIVKA-II levels were found in the vast majority of patients, indicating that vitamin $\mathrm{K}$ availability in the liver is too low to produce fully carboxylated clotting factors. The high dp-ucMGP levels found in all patients confirm our previous findings in HD patients and indicate a poor vascular vitamin $\mathrm{K}$ status. Plasma dp-ucMGP was the only marker that correlated with PIVKA-II levels, stressing its value as a vascular biomarker for vitamin $\mathrm{K}$ status. Thus both hepatic as well as extra-hepatic vitamin $\mathrm{K}$ status were strikingly poor. 
In general, dietary vitamin $\mathrm{K}$ intake in healthy subjects and in HD patients is sufficient to maintain normal haemostasis, but may not be sufficient for full carboxylation (activation) of the extra-hepatic Gla-proteins. Our study confirms that the dietary vitamin $\mathrm{K}$ intake in $\mathrm{HD}$ patients is low, although we could not demonstrate a correlation between vitamin $\mathrm{K}$ intake and markers for vitamin $\mathrm{K}$ status. The high dp-ucMGP levels are strongly suggestive for vascular vitamin $\mathrm{K}$ insuffiency to a level that the calcification-inhibitory activity of MGP is impaired, which may contribute to the extremely high risk for arterial calcification in HD patients. These data therefore warrant vitamin $\mathrm{K}$ intervention studies to elucidate the potential clinical benefit of vitamin $\mathrm{K}$ supplementation in HD patients.

In conclusion, vascular calcification is a major determinant of cardiovascular mortality and one of the main calcification inhibitors in the vasculature is MGP. The measurement of biomarkers, which can reflect the early signs of vascular disease, could be of great importance. We have developed three different MGP assays to measure various circulating MGP species (Chapter 8). The t-ucMGP assay has the potential to become a biomarker for prevalent arterial calcification, although this has to be further confirmed in ESRD and other patient populations. The dp-ucMGP assay seems to be particularly suited to assess vascular vitamin $\mathrm{K}$ status and may therefore contribute to cardiovascular risk assessment. In conclusion, based on our results, each MGP assay may have its own merit for research and/or for diagnostic use. Further research is necessary to investigate the full diagnostic potential of the different MGP assays. 


\section{Curriculum vitae}

Ellen Cranenburg was born on the $22^{\text {th }}$ of January 1982 in Boxmeer. She completed high school (VWO Gymnasium) at Maurick College Vught in 2000. She moved to Maastricht in the same year to study Medicine at Maastricht University. During her study she was assigned as student assistant to the Department of Anatomy / Embryology of the Faculty of Medicine were she assisted in practical lessons for students. In the $4^{\text {th }}$ year of her study, Ellen was introduced to dr. Cees Vermeer, which eventually resulted in a 3-month research project under his supervision in 2006. In the same year, she spent her last clinical internship of 3 months at the division of Rheumatology at the Academic Hospital Maastricht, which was her first introduction to this specialization. In August 2006 she graduated from her study Medicine and started her work in September of that year on her PhD thesis at the Department of Biochemistry within VitaK under supervision of dr. Cees Vermeer, dr. Leon Schurgers, and prof. dr. Jan Rosing. Her thesis describes several studies in relation to the role of circulating Matrix Gla-protein as a biomarker for vascular disease. Ellen started her pre-training in Internal Medicine for her specialization in Rheumatology in August 2010 at Máxima Medisch Centrum in Veldhoven, under supervision of dr. Harm Haak. She lives together with Arthur Denissen in Middelbeers (Noord-Brabant). 


\section{Publications}

Vitamin K: the coagulation vitamin that became omnipotent.

Cranenburg EC, Schurgers LJ, Vermeer C

Thromb Haemost 2007; 98(1):120-5

The circulating inactive form of matrix Gla Protein (ucMGP) as a biomarker for cardiovascular calcification.

Cranenburg EC, Vermeer C, Koos R, Boumans ML, Hackeng TM, Bouwman FG, Kwaijtaal M, Brandenburg VM, Ketteler M, Schurgers LJ

J Vasc Res 2008; 45(5): 427-36

Matrix Gla-protein: the calcification inhibitor in need of vitamin K.

Schurgers LJ, Cranenburg EC, Vermeer C

Thromb Haemost 2008; 100(4): 593-603

Uncarboxylated matrix Gla protein (ucMGP) is associated with coronary artery calcification in hemodialysis patients.

Cranenburg EC, Brandenburg VM, Vermeer C, Stenger M, Mühlenbruch G, Mahnken AH, Gladziwa U, Ketteler M, Schurgers LJ

Thromb Haemost 2009; 101(2): 359-66

Association of kidney function and uncarboxylated matrix Gla protein: data from the Heart and Soul study.

Parker BD, Ix JH, Cranenburg EC, Vermeer C, Whooley MA, Schurgers LJ

Nephrol Dial Transplant 2009; 24(7): 2095-101

Relation of circulating Matrix Gla-Protein and anticoagulation status in patients with aortic valve calcification.

Koos R, Krueger T, Westenfeld R, Kühl HP, Brandenburg V, Mahnken AH, Stanzel S, Vermeer C, Cranenburg EC, Floege J, Kelm M, Schurgers LJ

Thromb Haemost 2009; 101(4): 706-13

Characterisation and potential diagnostic value of circulating matrix Gla protein (MGP) species.

Cranenburg EC, Koos R, Schurgers LJ, Magdeleyns EJ, Schoonbrood TH, Landewé RB, Brandenburg VM, Bekers O, Vermeer C

Thromb Haemost 2010; 104(4): 811-22 
Hydrolysed formula is a risk factor for vitamin $\mathrm{K}$ deficiency in infants with unrecognised cholestasis.

van Hasselt PM, de Vries W, de Vries E, Kok K, Cranenburg EC, de Koning TJ, Schurgers L, Verkade HJ, Houwen RH

J Pediatr Gastroenterol Nutr 2010; 51(6): 773-6

Progression of aortic calcification is associated with disorders of mineral metabolism and mortality in chronic dialysis patients.

Noordzij M, Cranenburg EC, Engelsman LF, Hermans MM, Boeschoten EW, Brandenburg VB, Bos WJW, Kooman JP, Dekker FW, Ketteler M, Schurgers LJ, Krediet RT, Korevaar JC Nephrol Dial Transplant, 2010

Circulating total-uncarboxylated Matrix Gla-protein (t-ucMGP) is not associated with mortality in dialysis patients

Cranenburg EC, Noordzij M, Schurgers LJ, Kooman JP, Hermans M, Vermeer C, Boeschoten EW, Dekker FW, Verduijn M

Submitted

Rare mutations in the gene encoding matrix Gla-protein (MGP): a new case of Keutel syndrome serves as a model for exploring MGP functions in humans.

Cranenburg EC, van Spaendonck-Zwarts KY, Bonafe L, Rödiger LA, Dikkers FG, van Essen AJ, Superti-Furga A, Alexandrakis A, Vermeer C, Schurgers LJ, Laverman GD

Submitted

Vitamin $\mathrm{K}$ intake and (hepatic and extra-hepatic) vitamin $\mathrm{K}$ status in hemodialysis patients: a pilot study.

Cranenburg EC, Schurgers LJ, Uiterwijk H, Beulens JW, Dalmeijer GW, Westerhuis R, Magdeleyns EJ, Herfs M, Vermeer C, Laverman GD

Submitted

Severe functional vitamin K deficiency in HD patients is significantly improved by vitamin K2 supplementation.

Westenfeld R, Krueger T, Schlieper G, Cranenburg EC, Magdeleyns EJ, Heidenreich S, Holzmann S, Vermeer C, Jahnen-Dechent W, Ketteler M, Floege J, Schurgers L Submitted

Circulating desphoso-carboxylated Matrix Gla Protein predicts survival in HD patients.

Schlieper G, Krüger T, Cranenburg EC, Brandenburg VM, Djuric Z, Damjanovic T, Westenfeld R, Kettler M, Vermeer C, Dimkovic N, Floege J, Schurgers LJ.

Submitted 


\section{Dankwoord}

De eerste regels van mijn dankwoord wil ik graag wijden aan mijn team van copromotores: Dr. C. Vermeer en dr. L. J. Schurgers. Cees en Leon, jullie zijn voor mij een uniek team geweest, met als resultaat een meer dan uitstekende begeleiding voor mij!

Cees, voor jou ben ik waarschijnlijk een van de laatste promovenda op jouw lange lijst. Een hele diverse lijst, waarop verschillende dokters mij zijn voorgegaan. Je hebt mij en mijn "klinische blik" dan ook altijd goed begrepen. Ik ben je ontzettend dankbaar voor de kans die je me hebt gegeven om dit promotieonderzoek bij VitaK te gaan doen en voor de vrijheid die ik vervolgens kreeg om dit grotendeels naar eigen inzicht in te vullen. Hierbij stond jij mij altijd met raad en daad terzijde. Je leerde me om een eigen mening te vormen en deze te beargumenteren. Menig discussie volgde vervolgens, maar altijd met wederzijds respect. Respect van mijn kant voor de eigenzinnige wijze waarop jij onderzoek doet en je volhardendheid. Ik wens jou nog heel veel fijne onderzoeksjaren en wandeljaren samen met Hélène.

Leon, voor jou was ik de eerste promovenda op de lange lijst die nog gaat komen. Ontelbare keren nam jij de tijd om de zaken voor mij op een rij te zetten met behulp van tekeningen, schema's en tabellen. Want biochemie en cellen, dat vond ik toch wel lastig! Vanaf het eerste begin tot aan het einde nu, is er niet één keer geweest dat je geen tijd voor me had. Tijdens deze gesprekken wist je me ook altijd weer te enthousiasmeren en motiveren met de grote passie die jij voor de wetenschap hebt. En laten we onze reisjes naar congressen niet vergeten, waarbij je me voorstelde aan ontelbaar veel collega's . Dit eindigde vervolgens met kaasfondue in Genève en hamburgers in Philadelphia - alles in kader van de wetenschap natuurlijk. Hier denk ik met veel plezier aan terug. Ik wens jou nog zeer veel jaren met interessant onderzoek en natuurlijk met je gezin.

Zonder twijfel zijn mijn paranimfen de beste collega's die iemand zich wensen kan.

Kirsten, wij konden het samen meteen goed vinden. Over van alles konden wij samen praten en lachen, waarbij je me ook nog de Limburgse cultuur probeerde bij te brengen (vooral veel vlaai eten, volgens mij). In de loop van de jaren ben je naast mijn collega ook een goede vriendin geworden, bij wie ik altijd terecht kon als het me wat minder ging. Heel veel dank daarvoor! Ik wens jou en Rob een heel fijn leven samen in het diepe zuiden. 
Elke, het duurde even voor ik het doorhad, maar onder al dat zwart schuilt een hart van goud! Altijd kon ik ook bij jou terecht voor een luisterend oor, een kop thee en natuurlijk wat lekkers daarbij. En wat kon je mij aan het lachen maken met je ongelooflijke gevoel voor humor. Samen waren wij de "MGP-diehards"! Blijf samen met Stefan nog lang genieten van de goede dingen des levens.

Lieve meiden, bedankt dat jullie mijn paranimfen willen zijn!

Lavienja, fijne jaren hebben wij samen op één kamer doorgebracht, waarin we o.a. de belangrijkste tv-persoonlijkheden doorspraken (variërend van boeren tot vrouwen uit het Gooi). Maar discussies over het werk schuwden we ook niet! Ik heb een groot respect voor de manier waarop jouw werk als wetenschapper en als zakenvrouw combineert met je gezin. Ik wens je vooral fijne jaren met je dochters en je man toe.

Elke, ik heb jou leren kennen als een heel lieve en betrouwbare collega. Je hebt me heel veel geholpen met mijn promotie en proefschrift vanuit je eigen ervaringen. Ik heb me altijd door jou enorm gesteund gevoeld, dank daarvoor. Ik wens je heel veel mooie reizen toe!

Marjolein, dank voor alle hulp o.a. bij de immunohistochemie; wat is daar veel werk in gaan zitten! Ook bij jou kon ik altijd terecht; ik wens jou en Wouter alle moois voor de toekomst.

Marjo, altijd ben jij geïnteresseerd en medelevend, goede eigenschappen voor een collega! Ik wens jou ook een mooi proefschrift toe.

Nadja, je bent een heel lieve collega voor me geweest op de $1^{\mathrm{e}}$ verdieping, dank daarvoor. Ik wens jou alle goeds voor de toekomst!

Peggy, Noura en Stefan, jullie wens ik nog veel leuke werkjaren bij VitaK toe!

Tot slot wil ik alle collega's van VitaK bedanken voor het vele werk dat zij verricht hebben in (en buiten) het laboratorium voor de onderzoeken beschreven in dit proefschrift. Daarbij wil ik Marie-Louise Boumans niet vergeten; dank voor al je hulp en werk tijdens de eerste jaren!

De leden van de beoordelingscommissie, Prof. dr. H. ten Cate, Prof. dr. A. Bast, Prof. dr. J. Flöge, Prof. dr. J.M.J.P. van der Linden en Prof. dr. Y.T. van der Schouw wil ik bedanken voor de beoordeling van dit proefschrift. Prof. dr. J. Flöge, thank you for the critical evaluation of my thesis.

Prof. dr. Jan Rosing wil ik bedanken voor het kritisch doornemen van mijn artikelen en de tijd die hij voor mij vrijmaakte. 
Prof. dr. Hackeng, beste Tilman, dank voor de prettige samenwerking bij het onderzoek naar circulerend MGP.

Dr. Henri Spronk en René van Oerle, dank voor onze goede samenwerking bij het verzamelen van bloedmonsters van gezonde proefpersonen.

Beste Trees, dank voor alle hulp in de loop van de jaren!

Graag wil ik alle andere collega's en (oud) AIO's van Biochemie bedanken voor mijn fijne tijd daar en voor de interesse in mijn onderzoek!

Dr. Brandenburg, dear Vincent, many thanks for our collaboration on the t-ucMGP studies. I really enjoyed our discussions and your enthusiasm for MGP and vitamin K.

Prof. dr. Markus Ketteler and dr. Ralf Koos, I would also like to thank you for our collaboration on several studies and your interest in circulating MGP.

Dr. Verduijn, beste Marion, veel dank voor alle hulp bij de statische analyse van de MGP resultaten van het NECOSAD cohort. Tevens dank aan de andere leden van NECOSAD, in het bijzonder dr. Els Boeschoten en dr. Friedo Dekker voor het beschikbaar stellen van de samples en onze nuttige discussies.

Zonder dr. Jeroen Kooman en dr. Marc Hermans zouden deze metingen niet tot stand zijn gekomen, dank daarvoor.

Dr. Noordzij, beste Marlies, dank voor onze prettige samenwerking voor de NECOSAD studies en hulp bij de statische analyse.

Drs. Schoonbrood, beste Thea, samen hebben wij het onderzoek naar MGP in patiënten met reumatische aandoeningen opgezet. Een hele fijne samenwerking waarin jij mij goed begeleid hebt. Daarnaast heb jij mij lang geleden samen met Simone geënthousiasmeerd voor het vak van reumatoloog. Het heeft resultaat gehad!

Dr. Robert Landewé, dank voor de hulp bij het uitwerken van bovenstaand onderzoek.

Prof. Sjef van der Linden, veel dank voor de mogelijkheid om samen onderzoek te doen. Hierdoor bleef ik in contact met de reumatologie, wat later leidde tot de geweldige kans om in opleiding tot reumatoloog te komen. Dank voor het vertrouwen in mij.

Dr. Laverman, beste Goos, we leerden elkaar kennen in Philadelphia, en het diner tijdens de laatste congresavond leidde tot een vruchtbare samenwerking tussen het uiterste noorden en zuiden van Nederland! Dank voor alle tijd en energie die jij hierin gestoken hebt.

Lieve Herma, ons onderzoek leidde tot een prachtig afstuderen van jou! Dank voor de fijne samenwerking en ons prettige contact! Zonder jou hadden we dit onderzoek niet tot een goed einde kunnen brengen. 
Dr. van Spaendonck-Zwarts, beste Karin, een unieke patiënt leidde tot onze unieke samenwerking! Ik wil je bedanken voor het vertrouwen dat je in me had en alle tijd en energie die je in onze samenwerking hebt geïnvesteerd.

Dr. Beulens, beste Joline, we hebben samen gewerkt aan de registratie van vitamine $\mathrm{K}$ inname. Een hele uitdaging voor mij, waarin je mij goed geholpen hebt.

Drs. Dalmeijer, beste Gerdien, dank voor je hulp bij dit onderzoek. Beiden dank voor de fijne samenwerking.

Dank aan alle patiënten en proefpersonen die participeerden aan mijn onderzoek.

Van het Biopartner Center Maastricht, waar ik op $1^{\mathrm{e}}$ verdieping veel fijne jaren gewerkt heb, wil ik Leon de Bruijn graag bedanken. Leon ("Strikje"), je hebt veel voor mij betekend. Niet alleen stond jij in praktische zin altijd voor mij klaar, maar je kon mij ook opbeuren als ik dat nodig had. Ik wens jou nog heel veel gelukkige jaren in topvorm! Georgia, bedankt voor je interesse in mij persoonlijk en in mijn onderzoek.

JveProducties, beste Jeroen, dank voor het maken van de mooie omslag en layout en alle geduld dat je daarbij met mij had.

Graag wil ik hier ook de specialisten en mijn collega arts-assistenten van de Interne Geneeskunde van het Máxima Medisch Centrum bedanken voor het warme welkom dat ik heb gekregen. De eerste periode na onderzoek weer terug in de kliniek is best zwaar, maar ook nu heb ik weer geweldige collega's getroffen. Kristof, mijn "mentor", bedankt voor de keren dat je me uit de brand hielp. Marij en Maren, bedankt voor het wegwijs maken in het MMC de eerste weken en al onze gesprekken daarna. Arno en Jacobien, Marten en Wouter, dank voor jullie goede supervisie en begeleiding in mijn eerste maanden terug in de kliniek. Dank aan de secretaresses en verpleegkundigen van 3C en 3D voor het geduld dat zij met mij hadden!

Dr. Harm Haak, mijn opleider, dank voor je vertrouwen in mij en de tijd die ik kreeg om mijn weg in de kliniek weer te vinden.

Zonder steun van familie en vrienden had ik niet zover kunnen komen.

Annemarie (Ari), wij zijn al heel lang vriendinnen en hebben heel veel leuke dingen gedaan samen; samen op vakantie, shoppen, naar musicals of gewoon theeleuten. Voor mij is dit de broodnodige ontspanning! Dank hiervoor en voor je geduld als ik wat minder tijd had!

Anne-Quirijne, vanaf de basisschool kennen we elkaar al en we gingen allebei Geneeskunde studeren en daarna promoveren, weliswaar op enige afstand van elkaar. 
Ondanks dat houden we gelukkig contact en is het als vanouds als we elkaar zien. Bij deze wil ik ook je vader dr. Paul Reuwer bedanken, die ons toch als eerste liet kennis maken met wetenschappelijk onderzoek!

Lieve andere "vriendinnen van het gym", we zien elkaar nog steeds regelmatig, waar ik altijd heel blij van word! Bedankt daarvoor!

Lieve Tonnie en Mari, mijn "schoonouders", jullie staan altijd klaar voor mij en Arthur. Niets is jullie te veel; zonder jullie hulp bij de verbouwing hadden we niet zo'n geweldig mooi huis gehad! En onze tuin ligt er dankzij Mari altijd picobello bij. Ik heb het geweldig getroffen met jullie.

Chantal en Günther, Sandra en Maikel, dank voor jullie interesse in mijn onderzoek en voor alle leuke momenten samen. Jullie kinderen zijn geweldig en maken mij altijd blij, ook in de meest drukke tijden. Dikke kus voor Estelle, Meeke, Keno, mijn petekind Fenne, en Jayden!

Lieve Kim, je bent echt een grote zus voor mij, die altijd voor me klaar staat. Ik ben heel trots op jou, je carrière, je huis en hoe je in het leven staat. Bedankt voor alles.

Remco, met jou kwam er heel veel hondenliefde en veel lekker eten mee. Samen met Laika, Senna, Boomer en Sem zullen we hopelijk nog veel wandelplezier beleven!

Dank ook aan de rest van de familie die altijd geïnteresseerd is in mijn wel en wee, vooral mijn Oma, die nog heel wat wijsheden in pacht heeft (zie stellingen)!

Lieve pap en mam, jullie hebben mij altijd gesteund in alles wat ik deed, op velerlei manieren. Zo hebben jullie mij vier keer helpen verhuizen, mij eindeloos vaak naar Maastricht gebracht, samen met Arthur ons hele huis verbouwd en daarna gepoetst, en nog veel meer. Ook kon ik jullie dag en nacht bellen als er iets was, en er was geen verjaardag dat jullie niet in Maastricht op de stoep stonden (desnoods om 7 uur 's ochtends). Pap, je bent een geweldige vader! Mam, ik ben heel trots op alles wat je bereikt hebt!

En tot slot, lieve Arthur, zonder jou was dit boekje nooit tot een goed einde gekomen. Sterker nog, dan had ik er niet aan durven beginnen; je hebt me vertrouwen in mezelf gegeven. Je staat altijd voor me klaar en zorgt heel goed voor mij. In 2010 liet je mijn dromen uitkomen met ons mooie huis en de bijbehorende beestenboel. Ik ben ontzettend trots op jou, je bedrijf, en alles wat we samen hebben. Je weet het, je bent "het" helemaal voor mij.

Ellen. 
PS: Een groot aantal dialysepatiënten heeft meegewerkt aan het onderzoek beschreven in dit proefschrift. Velen van hen wachten op een niertransplantatie. Zo wachtte ook mijn oom Martin Boons, totdat er helaas geen tijd meer was.

Staat $U$ positief tegenover orgaandonatie? Laat $U$ dan nu registreren als donor op www.donorregister.nl 